\title{
Access to Constrained Fluoropseudopeptides via Ring Closing Metathesis of Fluoroalkenes
}

David Guérin, ${ }^{\dagger}$ Isabelle Dez, ${ }^{\dagger}$ Annie-Claude Gaumont, ${ }^{\dagger}$ Xavier Pannecoucke, ${ }^{\ddagger}$ and Samuel Couve-Bonnaire*,

${ }^{\dagger}$ Normandie Univ., ENSICAEN, Unicaen, CNRS, LCMT, 14000 Caen, France

${ }^{*}$ Normandie Univ., INSA Rouen, UNIROUEN, CNRS, COBRA, 76000 Rouen, France

Supporting Information Placeholder

\section{Table of Contents}

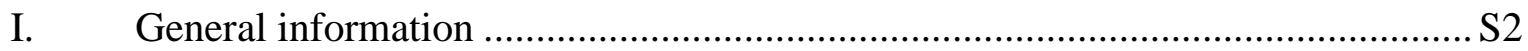

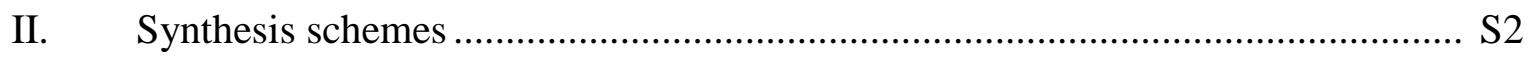

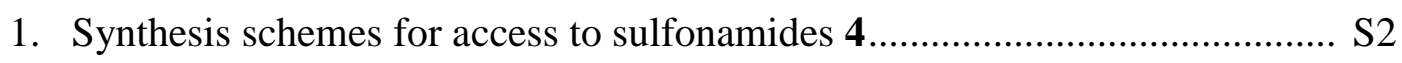

2. Details for other envisioned strategies …................................................. S3

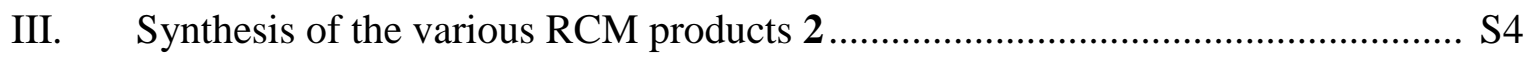

1. Experimental procedures and data ….......................................................... S4

a. Synthesis of sulfinimines 5 ........................................................ S4

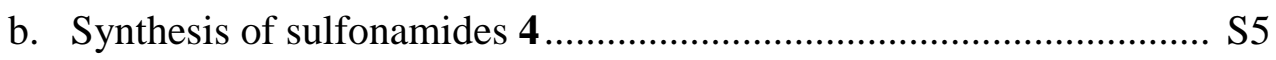

c. Synthesis of the bisolefins bearing secondary amide $\mathbf{9} \ldots \ldots \ldots \ldots \ldots \ldots . . . . . . . . .510$

d. Synthesis of the secondary PMB-amines 7 ..................................... S12

e. Synthesis of the PMB-protected bisolefins 3 ................................. S17

f. Synthesis of the cyclic lactams 2 through RCM ............................ S24

IV. Synthesis of the various fluoropseudopeptides 1 ......................................... S28

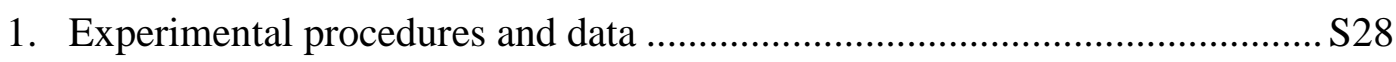

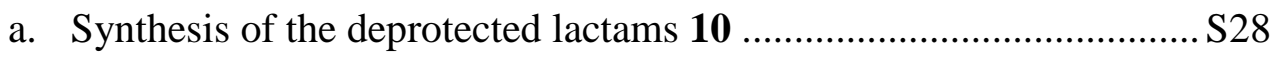

b. Synthesis of the fluoropseudopeptides 1 .........................................S30

V. $\quad{ }^{1} \mathrm{H},{ }^{19} \mathrm{~F}$ and ${ }^{13} \mathrm{C}$ Spectra for 1a,d, 2b-e,h, 3a-h, 4a-c, 5, 7 a-h, 9c,e,g, 10a,d ..... S32 


\section{General information}

All reactions have been carried out under inert atmosphere in pre-dried glassware. The dry common solvents used were purified by an Innovative Technology PureSolv purification system. Perfluorinated solvent: octafluorotoluene (FluoroChem), was directly used from commercial bottle and kept under argon atmosphere. Commercially available chemicals were obtained from Acros Organics, Aldrich Chemical, Strem Chemicals, Alfa Aesar, TCI, and used as received unless otherwise stated. All commercial chemicals were used without further purification. NMR spectra were recorded on a Bruker AC 400 spectrometer. Chemical shifts $(\delta)$ are quoted in ppm relative to TMS $\left({ }^{1} \mathrm{H}\right)$ and $\mathrm{CFCl}_{3}\left({ }^{19} \mathrm{~F}\right)$. Coupling constants $(J)$ are quoted in Hz. Microwave-assisted reactions were carried out into CEM Microwaves Discover (200 W). High-resolution mass spectra (HRMS) were recorded on Q-TOF Micro WATERS. Infrared spectra were recorded on a Spectrum One Perkin Elmer spectrophotometer. Enantiomeric excesses were determined by chiral HPLC equipped with a photodiode array detector (200-400 nm). See specific conditions detailed for each compound. In all cases, the analysis was calibrated with a sample of the racemate. The wave numbers $(v)$ of recorded IR-signals are quoted in $\mathrm{cm}^{-1}$. Optical rotations were recorded on an Atago AP - 100 automatic polarimeter at $589 \mathrm{~nm}$. Analytical thin layer chromatography was performed on precoated $200 \mu \mathrm{m}$ layer thickness silica gel 60 Alumigram $^{\circledast}$ Xtra SIL G/UV ${ }_{254}$. Visualization was accomplished with short wave UV light, potassium permanganate or phosphomolybdic acid staining solution followed by heating. Flash chromatography was either performed on Merck silica gel (40-63 mesh, solid deposit) by standard technique. Yields refer to chromatographically and spectroscopically pure compounds. The products $13^{[1]}, \mathbf{1 4}^{[2]}, \mathbf{1 5}^{[3]}$ have previously been described and were identified by comparison of their physical and spectroscopic data $\left({ }^{1} \mathrm{H},{ }^{19} \mathrm{~F}\right.$ and ${ }^{13} \mathrm{C}$ NMR $)$ with those in the cited references.

${ }^{[1]}$ Lemmonier, G.; Zoute, L.; Dupas, G.; Quirion, J.-C.; Jubault, P. J. Org. Chem. 2009, 74, 4124-4131.

${ }^{[2]}$ Zemmouri, R.; Kajjout, M.; Castanet, Y.; Eddarir, S.; Rolando, C. J. Org. Chem., 2011, 76, 7691-7698.

${ }^{[3]}$ Wheeler, P.; Vora H. U.; Rovis, T. Chem. Sci., 2013, 4, 1674.

\section{Synthesis Schemes}

1. Synthesis schemes for access to sulfinamides 4

The synthesis of the various sulfinamides $\mathbf{4}$ was carried out following a strategy previously described by our laboratory. ${ }^{[4]}$

The Ellman sulfinimine $\mathbf{5}$ was first synthesized through a four steps sequence starting from benzaldehyde $\mathbf{1 2}$.

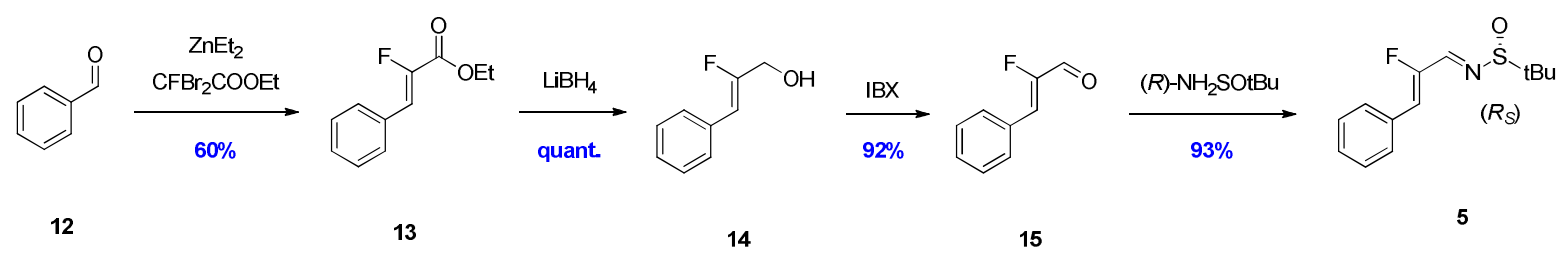

The primary alcohol $\mathbf{1 4}$ was obtained by reduction of the corresponding ethylic ester $\mathbf{1 3}$ and was submitted to IBX oxidation to afford aldehyde 15. Access to the Ellman sulfinimine $\mathbf{5}$ was possible through condensation of the chiral tert-butanesulfinamide to the aldehyde $\mathbf{1 5}$. (R)-tertbutanesulfinamide auxiliary was chosen in order to obtain major diastereomer with a newly created 
stereogenic center in $(S)$ configuration, as found in native peptides, by mean of Grignard reagent addition.

The results for the organometallic addition on compound $\mathbf{5}$ are listed below:

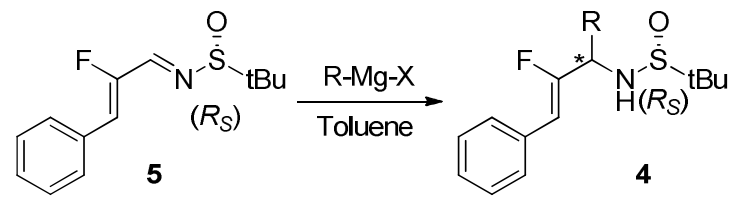

$\begin{array}{ccccccc}\text { Entry } & \mathrm{R} & \text { Imine } & \mathrm{X} & \text { Conditions } & \text { Yield of } \mathbf{4}(\%)^{\mathrm{a}} & \mathrm{dr}^{\mathrm{b}} \\ 1 & \mathrm{H} & (R)_{\mathrm{s}} \mathbf{5} & & & & \\ 2 & \mathrm{Me} & (R)_{\mathrm{s}} \mathbf{5} & \mathrm{Br} & 0^{\circ} \mathrm{C}, 40 \mathrm{~min} & 93 & 80 / 20 \\ 3 & \mathrm{Ph} & (R)_{\mathrm{s}} \mathbf{5} & \mathrm{Br} & -30^{\circ} \mathrm{C}, 2 \mathrm{~h} & 96 & 91 / 9 \\ 4 & \mathrm{i}-\mathrm{Pr} & (R)_{\mathrm{s}} \mathbf{5} & \mathrm{Cl} & 0^{\circ} \mathrm{C}, 1 \mathrm{~h} & 59 & 66 / 33 \\ 5 & \mathrm{Bn} & r a c-5 & \mathrm{Br} & 0^{\circ} \mathrm{C}, 2 \mathrm{~h} & 63 & 50 / 50\end{array}$

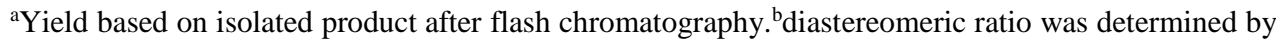
${ }^{19} \mathrm{~F}$ NMR.

Each diastereoisomer was isolated and engaged in the next steps of the sequence.

${ }^{[4]}$ Pierry, C.; Cahard, D.; Couve-Bonnaire, S.; Pannecoucke, X. Org. Biomol. Chem. 2011, 9, 2378.

2. Details for other envisioned strategies

As mentioned in the article, other protecting groups have been envisioned, instead of PMB group, in order to shortcut and/or facilitate the next steps of the synthesis.
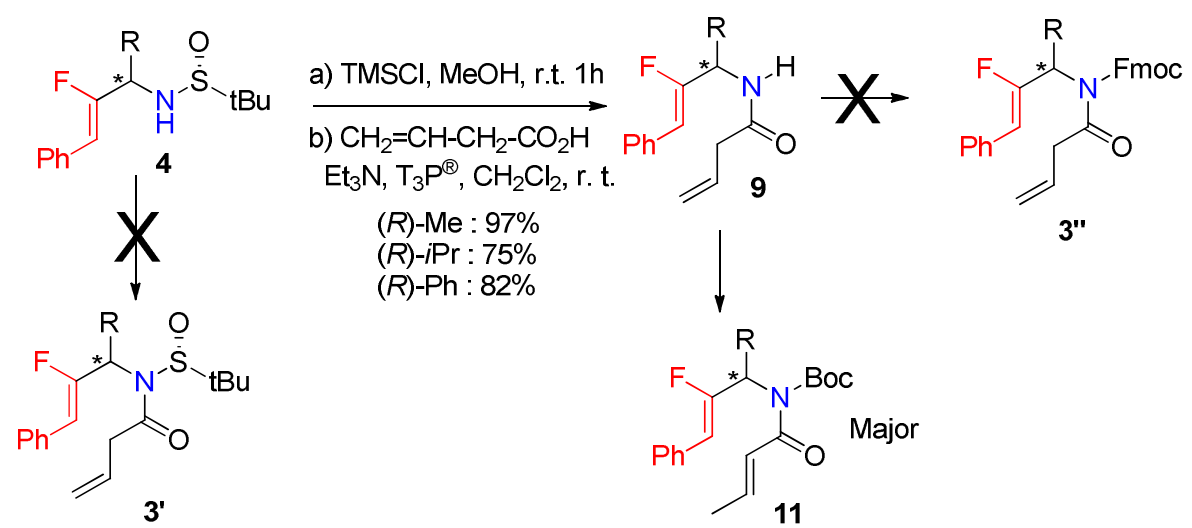

First, keeping the chiral auxiliary seemed to be interesting as it may be hydrolyzed during the last step of the synthesis sequence but unfortunately, various experimental conditions for the amide bond formation remained inefficient (compound 3'). So after formation of the amide bond (compound 9), $\mathrm{N}$ Fmoc protection in various experimental conditions proved to be unsuccessful (compound 3") and the formation of $N$-Boc resulted predominantly in an isomerization of the double bond leading to compound 11. 
III. Synthesis of the various RCM products 2

1. Experimental procedures and data

a. Synthesis of the sulfinimines $\mathbf{5}$

(Ss)-N-((Z)-2-fluoro-3-phenylallylidene)-2-methylpropane-2-sulfinamide (5)-S:

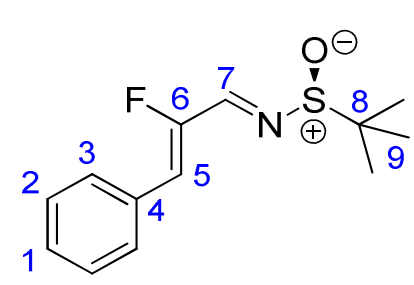

A $100 \mathrm{~mL}$ round bottom flask was equipped with a stir bar, a reflux condenser and flushed with argon. A solution of aldehyde X (1.2 g, 8 mmol, 1 eq.) in dry THF (36 mL) was prepared. Both Ti(OiPr $)_{4}(4.78 \mathrm{~mL}, 16$ mmol, 2 eq.) and (S)-tertbutylsulfinylamine (1.45 g, $12 \mathrm{mmol}, 1.5$ eq.) were added. The mixture was heated at reflux for $1 \mathrm{~h} 30$ and once cooled, poured into an equal volume of brine with rapid stirring. The resulting suspension was filtered through a pad of Celite ${ }^{\circledR}$ and the solid washed with EtOAc. The brine layer was extracted once with EtOAc. The combined organic portions were dried over $\mathrm{MgSO}_{4}$, filtered and concentrated under reduced pressure. The crude mixture was purified by chromatography on silica gel (Eluent: Pentane/Et $\left.{ }_{2} \mathrm{O}: 97 / 03\right)$ to afford (Ss)-N-((Z)-2-fluoro-3-phenylallylidene)-2-methylpropane-2-sulfinamide as a light yellow solid (1.99 g, $7.86 \mathrm{mmol}, 99 \%)$.

${ }^{1} \mathbf{H}$ NMR (400.13 MHz, CDCl$): \delta(\mathrm{ppm}) 8.10\left(\mathrm{~d},{ }^{3} J_{\mathrm{H}-\mathrm{F}}=19 \mathrm{~Hz}, 1 \mathrm{H}, \mathrm{H}_{7}\right), 7.69\left(\mathrm{~m}, 2 \mathrm{H}, \mathrm{H}_{3}\right), 7.42(\mathrm{~m}, 3 \mathrm{H}$, $\left.\mathrm{H}_{1,2}\right), 6.40\left(\mathrm{~d},{ }^{3} J_{\mathrm{H}-\mathrm{F}}=35 \mathrm{~Hz}, 1 \mathrm{H}, \mathrm{H}_{5}\right), 1.27\left(\mathrm{~s}, 9 \mathrm{H}, \mathrm{H}_{9}\right) .{ }^{19} \mathbf{F} \mathbf{N M R}\left(\mathbf{3 7 6 . 5 0} \mathbf{~ M H z}, \mathbf{C D C l}_{3}\right): \delta(\mathrm{ppm})-121.36$ $\left(\mathrm{dd},{ }^{3} J_{\mathrm{F}-\mathrm{H}}=19 \mathrm{~Hz},{ }^{3} J_{\mathrm{F}-\mathrm{H}}=35 \mathrm{~Hz}, 1 \mathrm{~F}, \mathrm{~F}_{6}\right) .{ }^{13} \mathbf{C} \mathbf{N M R}\left(\mathbf{7 5 . 4 7} \mathbf{M H z}, \mathbf{C D C l}_{3}\right): \delta(\mathrm{ppm}) 155.4\left(\mathrm{~d},{ }^{2} J_{\mathrm{C}-\mathrm{F}}=21 \mathrm{~Hz}\right.$, $\left.1 \mathrm{C}, \mathrm{C}_{7}\right), 154.2\left(\mathrm{~d},{ }^{1} J_{\mathrm{C}-\mathrm{F}}=264 \mathrm{~Hz}, 1 \mathrm{C}, \mathrm{C}_{6}\right), 131.7\left(\mathrm{~d},{ }^{3} J_{\mathrm{C}-\mathrm{F}}=4 \mathrm{~Hz}, 1 \mathrm{C}, \mathrm{C}_{4}\right), 130.2\left(\mathrm{~s}, 2 \mathrm{C}, \mathrm{C}_{2}\right), 128.9(\mathrm{~s}, 2 \mathrm{C}$, $\left.\mathrm{C}_{3}\right), 129.9\left(\mathrm{~d},{ }^{6} J_{\mathrm{C}-\mathrm{F}}=2 \mathrm{~Hz}, 1 \mathrm{C}, \mathrm{C}_{1}\right), 122.8\left(\mathrm{~d},{ }^{2} J_{\mathrm{C}-\mathrm{F}}=6 \mathrm{~Hz}, 1 \mathrm{C}, \mathrm{C}_{5}\right), 58.3\left(\mathrm{~s}, 1 \mathrm{C}, \mathrm{C}_{8}\right), 22.5\left(\mathrm{~s}, 3 \mathrm{C}, \mathrm{C}_{9}\right) . \mathbf{I R}$ (neat) $v 2981,1643,1571,1448,1369,1180,1075,977,928,906,879,837,782,760,687,570 \mathrm{~cm}^{-1}$. HR-MS (ESI+): $\mathrm{m} / \mathrm{z}=254.1025[\mathrm{M}+\mathrm{H}]^{+}$, calcd. for $\mathrm{C}_{13} \mathrm{H}_{17} \mathrm{FNOS}: 254.1015$. Anal. calculated for $\mathbf{C}_{13} \mathbf{H}_{16}$ FNOS: C, 61.63; H, 6.37; N, 5.53; S, 12.66. Found: C, 61.63; H, 6.35; N, 5.52; S, 12.68. $R f=$ $0.39\left(5 \% \mathrm{Et}_{2} \mathrm{O}\right.$ in pentane $)$.

$\left(\boldsymbol{R}_{S}\right)-\mathrm{N}-((\mathrm{Z})-2-f l u o r o-3-p h e n y l a l l y l i d e n e)-2-m e t h y l p r o p a n e-2-s u l f i n a m i d e(5)-R$ :

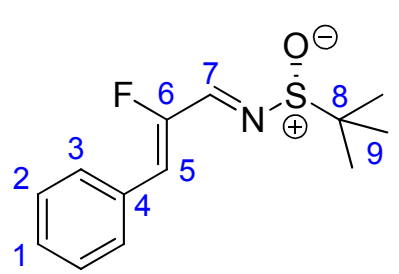

A $100 \mathrm{~mL}$ round bottom flask was equipped with a stir bar, a reflux condenser and flushed with argon. A solution of aldehyde X (300 mg, 2 mmol, 1 eq.) in dry THF (9 mL) was prepared. Both Ti(OiPr $)_{4}(1.2 \mathrm{~mL}, 4$ mmol, 2 eq.) and ( $R$ )-tertbutylsulfinylamine ( $363 \mathrm{mg}, 3 \mathrm{mmol}, 1.5$ eq.) were added. The mixture was heated at reflux for $1 \mathrm{~h} 30$ and once cooled, poured 
into an equal volume of brine with rapid stirring. The resulting suspension was filtered through a pad of Celite ${ }^{\circledR}$ and the solid washed with EtOAc. The brine layer was extracted once with EtOAc. The combined organic portions were dried over $\mathrm{MgSO}_{4}$, filtered and concentrated under reduced pressure. The crude mixture was purified by chromatography on silica gel (Eluent: Pentane/Et $\left.{ }_{2} \mathrm{O}: 97 / 03\right)$ to afford (Rs)-N-((Z)-2-fluoro-3-phenylallylidene)-2-methylpropane-2-sulfinamide as a white solid (470 mg, $1.86 \mathrm{mmol}, 93 \%)$.

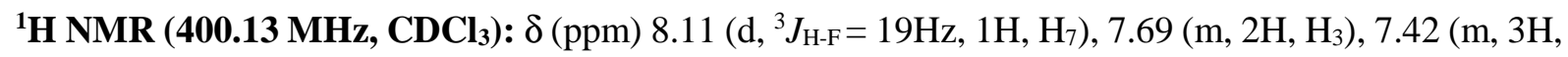
$\left.\mathrm{H}_{1,2}\right), 6.40\left(\mathrm{~d},{ }^{3} J_{\mathrm{H}-\mathrm{F}}=35 \mathrm{~Hz}, 1 \mathrm{H}, \mathrm{H}_{5}\right), 1.28\left(\mathrm{~s}, 9 \mathrm{H}, \mathrm{H}_{9}\right) .{ }^{19} \mathbf{F}$ NMR (376.50 $\left.\mathbf{M H z}, \mathbf{C D C l}_{3}\right): \delta(\mathrm{ppm})-$ $121.39\left(\mathrm{dd},{ }^{3} J_{\mathrm{F}-\mathrm{H}}=19 \mathrm{~Hz},{ }^{3} J_{\mathrm{F}-\mathrm{H}}=34 \mathrm{~Hz}, 1 \mathrm{~F}, \mathrm{~F}_{6}\right) .{ }^{13} \mathbf{C} \mathbf{N M R}\left(\mathbf{7 5 . 4 7} \mathbf{~ M H z}, \mathbf{C D C l}_{3}\right): \delta(\mathrm{ppm}) 155.4\left(\mathrm{~d},{ }^{2} J_{\mathrm{C}-}\right.$ $\left.\mathrm{F}=20 \mathrm{~Hz}, 1 \mathrm{C}, \mathrm{C}_{7}\right), 154.2\left(\mathrm{~d},{ }^{1} J_{\mathrm{C}-\mathrm{F}}=265 \mathrm{~Hz}, 1 \mathrm{C}, \mathrm{C}_{6}\right), 131.7\left(\mathrm{~d},{ }^{3} J_{\mathrm{C}-\mathrm{F}}=4 \mathrm{~Hz}, 1 \mathrm{C}, \mathrm{C}_{4}\right), 130.3\left(\mathrm{~s}, 2 \mathrm{C}, \mathrm{C}_{2}\right)$, $130.0\left(\mathrm{~d},{ }^{6} J_{\mathrm{C}-\mathrm{F}}=2 \mathrm{~Hz}, 1 \mathrm{C}, \mathrm{C}_{1}\right), 128.9\left(\mathrm{~s}, 2 \mathrm{C}, \mathrm{C}_{3}\right), 122.9\left(\mathrm{~d},{ }^{2} J_{\mathrm{C}-\mathrm{F}}=6 \mathrm{~Hz}, 1 \mathrm{C}, \mathrm{C}_{5}\right), 58.2\left(\mathrm{~s}, 1 \mathrm{C}, \mathrm{C}_{8}\right), 22.5(\mathrm{~s}$, 3C, C9). IR (neat) v 2979, 1715, 1643, 1571, 1449, 1365, 1294, 1256, 1183, 1128, 1077, 980, 928, 906, 828, 762, 758, $687 \mathrm{~cm}^{-1}$. HR-MS (ESI+): $\mathrm{m} / \mathrm{z}=254.1027[\mathrm{M}+\mathrm{H}]^{+}$, calcd. for $\mathrm{C}_{13} \mathrm{H}_{17} \mathrm{FNOS}: 254.1015$. Anal. calculated for $\mathbf{C}_{13} \mathbf{H}_{16}$ FNOS: C, 61.63; H, 6.37; N, 5.53; S, 12.66. Found: C, 61.67; H, 6.37; N, 5.55; $\mathrm{S}, 12.66 . R f=0.41\left(5 \% \mathrm{Et}_{2} \mathrm{O}\right.$ in pentane).

\section{b. Synthesis of the sulfinamides 4}

\section{General Procedure for the synthesis of the sulfinamides by nucleophilic addition:}

The $\beta$-Fluoroenimine ( 1 eq.) and (depending on the case) a $2 \mathrm{M}$ solution of trimethylaluminium (1.1 eq.) were placed in a round-bottom flask under argon atmosphere and dissolved in anhydrous toluene. The solution was cooled down to the reported temperature and a solution of the alkylmagnesium halide was slowly added. The solution was stirred at the reported temperature and the progress of the reaction monitored through ${ }^{19} \mathrm{~F}$ NMR. After completion of the reaction, the solution was quenched with a saturated solution of $\mathrm{NH}_{4} \mathrm{Cl}$ and extracted three times with EtOAc. The combined organic layers were then washed with brine, dried over $\mathrm{MgSO}_{4}$, filtered and concentrated under reduced pressure. The crude residue was checked by ${ }^{19} \mathrm{~F}$ NMR for determination of diastereomeric ratio and purified by chromatography on silica gel (eluent: pentane/Et $2 \mathrm{O})$ to afford the desired sulfinamides $(S, R \mathrm{~s})$ and $(R, R \mathrm{~s})$ separately. 
$\left.\left(R_{S}\right)-N-(Z)-3-f l u o r o-4-p h e n y l b u t-3-e n-2-y l\right)-2-m e t h y l p r o p a n e-2-s u l f i n a m i d e(4 b-c)$ :

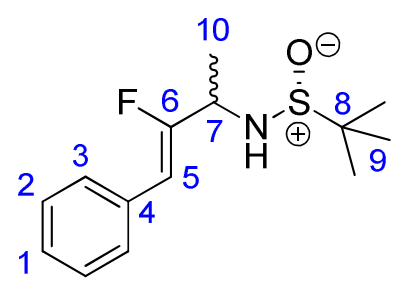

The general procedure for synthesis of the sulfonamides using $(R \mathrm{~s})-\beta$ Fluoroenimine (1 eq., $530 \mathrm{mg}, 2.1 \mathrm{mmol}$ ) and a solution of trimethylaluminium (1.1 eq., $1.15 \mathrm{~mL}$ of a $2 \mathrm{M}$ solution, $2.3 \mathrm{mmol}$ ) in toluene $(14 \mathrm{~mL})$ followed by flash chromatography on silica gel (eluent: pentane/Et ${ }_{2} \mathrm{O}$ 80/20) afforded $\boldsymbol{N}$-((R,Z)-3-fluoro-4-phenylbut-3-en-2-yl)-2-

methylpropane-2-sulfinamide (523 mg, $1.9 \mathrm{mmol}, 93 \%$ ) as: a white crystalline solid (420 mg, 1.6 mmol, yield $75 \%$ ) for diastereomer ( $S, R \mathrm{~s})$ and a colorless thick oil (103 mg, $0.4 \mathrm{mmol}$, yield $18 \%$ ) for diastereomer $(R, R \mathrm{~s})$.

\section{$\underline{(S, R s) \text { diastereoisomer }}(4 b)$ :}

${ }^{1} \mathrm{H}$ NMR (400.13 MHz, $\left.\mathbf{C D C l}_{3}\right): \delta(\mathrm{ppm}) 7.36\left(\mathrm{~m}, 5 \mathrm{H}, \mathrm{H}_{1,2,3}\right), 5.74\left(\mathrm{~d},{ }^{3} J_{\mathrm{H}-\mathrm{F}}=39 \mathrm{~Hz}, 1 \mathrm{H}, \mathrm{H}_{5}\right), 4.16(\mathrm{~m}$, $\left.1 \mathrm{H}, \mathrm{H}_{7}\right), 3.17\left(\mathrm{~d},{ }^{3} J_{\mathrm{H}-\mathrm{H}}=5 \mathrm{~Hz}, 1 \mathrm{H}, \mathrm{NH}\right), 1.53\left(\mathrm{~d},{ }^{3} J_{\mathrm{H}-\mathrm{H}}=7 \mathrm{~Hz}, 3 \mathrm{H}, \mathrm{H}_{10}\right), 1.24\left(\mathrm{~s}, 9 \mathrm{H}, \mathrm{H}_{9}\right) .{ }^{19} \mathbf{F} \mathbf{~ N M R}$ (376.50 MHz, $\left.\mathbf{C D C l}_{3}\right): \delta(\mathrm{ppm})-116.73\left(\mathrm{dd},{ }^{3} J_{\mathrm{F}-\mathrm{H}}=18 \mathrm{~Hz},{ }^{3} J_{\mathrm{F}-\mathrm{H}}=38 \mathrm{~Hz}, 1 \mathrm{~F}, \mathrm{~F}_{6}\right) .{ }^{\mathbf{1 3}} \mathbf{C}$ NMR (75.47 MHz, $\left.\mathbf{C D C l}_{3}\right): \delta(\mathrm{ppm}) 159.1\left(\mathrm{~d},{ }^{1} J_{\mathrm{C}-\mathrm{F}}=270 \mathrm{~Hz}, 1 \mathrm{C}, \mathrm{C}_{6}\right), 132.7\left(\mathrm{~d},{ }^{3} J_{\mathrm{C}-\mathrm{F}}=2 \mathrm{~Hz}, 1 \mathrm{C}, \mathrm{C}_{4}\right), 128.7\left(\mathrm{~s}, 2 \mathrm{C}, \mathrm{C}_{2}\right), 128.5$ $\left(\mathrm{s}, 2 \mathrm{C}, \mathrm{C}_{3}\right), 127.5\left(\mathrm{~d},{ }^{6} J_{\mathrm{C}-\mathrm{F}}=2 \mathrm{~Hz}, 1 \mathrm{C}, \mathrm{C}_{1}\right), 107.1\left(\mathrm{~d},{ }^{2} J_{\mathrm{C}-\mathrm{F}}=7 \mathrm{~Hz}, 1 \mathrm{C}, \mathrm{C}_{5}\right), 55.9\left(\mathrm{~s}, 1 \mathrm{C}, \mathrm{C}_{8}\right), 53.3\left(\mathrm{~d},{ }^{2} J_{\mathrm{C}-\mathrm{F}}\right.$ $\left.=28 \mathrm{~Hz}, 1 \mathrm{C}, \mathrm{C}_{7}\right), 22.5\left(\mathrm{~s}, 3 \mathrm{C}, \mathrm{C}_{9}\right), 20.4\left(\mathrm{~d},{ }^{3} \mathrm{~J}_{\mathrm{C}-\mathrm{F}}=2 \mathrm{~Hz}, 1 \mathrm{C}, \mathrm{C}_{10}\right)$. IR (neat) $v 3200,2980,1684,1448$, 1366, 1309, 1284, 1172, 1111, 1084, 1053, 1028, 1010, 932, 911, 881, 856, 843, 753, $693 \mathrm{~cm}^{-1}$. HRMS (ESI+): $\mathrm{m} / \mathrm{z}=270.1330[\mathrm{M}+\mathrm{H}]^{+}$, calcd. for $\mathrm{C}_{14} \mathrm{H}_{21} \mathrm{FNOS}$ : 270.1328. Anal. calculated for $\mathbf{C}_{14} \mathbf{H}_{20}$ FNOS: C, 62.42; H, 7.48; N, 5.20; S, 11.90 Found: C, 62.37; H, 7.51; N, 5.17; S, 11.91. [ $\left.\alpha\right]_{D}^{25}=$ $-99\left(c 1.5, \mathrm{CH}_{3} \mathrm{Cl}\right) . R f=0.17\left(20 \% \mathrm{Et}_{2} \mathrm{O}\right.$ in pentane $)$.

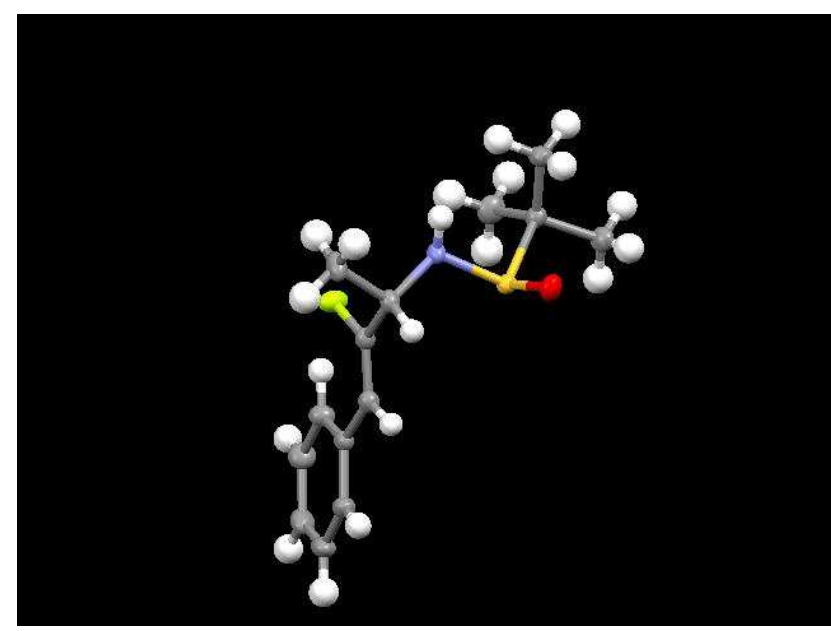

CCDC number: 1481673

$\underline{(R, R s) \text { diastereoisomer }}(4 c)$ :

${ }^{1} \mathbf{H}$ NMR (400.13 MHz, CDCl $)$ : $\delta(\mathrm{ppm}) 7.32\left(\mathrm{~m}, 5 \mathrm{H}, \mathrm{H}_{1,2,3}\right), 5.78\left(\mathrm{~d},{ }^{3} J_{\mathrm{H}-\mathrm{F}}=39 \mathrm{~Hz}, 1 \mathrm{H}, \mathrm{H}_{5}\right), 4.10(\mathrm{~m}$, $\left.1 \mathrm{H}, \mathrm{H}_{7}\right), 3.55\left(\mathrm{~d},{ }^{3} J_{\mathrm{H}-\mathrm{H}}=5 \mathrm{~Hz}, 1 \mathrm{H}, \mathbf{N H}\right), 1.48\left(\mathrm{~d},{ }^{3} \mathrm{~J}_{\mathrm{H}-\mathrm{H}}=7 \mathrm{~Hz}, 3 \mathrm{H}, \mathrm{H}_{10}\right), 1.23\left(\mathrm{~s}, 9 \mathrm{H}, \mathrm{H}_{9}\right) .{ }^{19} \mathbf{F} \mathbf{~ N M R}$ 
(376.50 MHz, $\left.\mathbf{C D C l}_{3}\right): \delta(\mathrm{ppm})-116.05\left(\mathrm{dd},{ }^{3} J_{\mathrm{F}-\mathrm{H}}=14 \mathrm{~Hz},{ }^{3} \mathrm{~J}_{\mathrm{F}-\mathrm{H}}=39 \mathrm{~Hz}, 1 \mathrm{~F}, \mathrm{~F}_{6}\right) .{ }^{13} \mathbf{C}$ NMR (75.47 MHz, $\left.\mathbf{C D C l}_{3}\right): \delta(\mathrm{ppm}) 159.6\left(\mathrm{~d},{ }^{1} J_{\mathrm{C}-\mathrm{F}}=269 \mathrm{~Hz}, 1 \mathrm{C}, \mathrm{C}_{6}\right), 132.5\left(\mathrm{~d},{ }^{3} J_{\mathrm{C}-\mathrm{F}}=2 \mathrm{~Hz}, 1 \mathrm{C}, \mathrm{C}_{4}\right), 128.6\left(\mathrm{~s}, 2 \mathrm{C}, \mathrm{C}_{2}\right), 128.3$ $\left(\mathrm{s}, 2 \mathrm{C}, \mathrm{C}_{3}\right), 127.3\left(\mathrm{~d},{ }^{6} J_{\mathrm{C}-\mathrm{F}}=2 \mathrm{~Hz}, 1 \mathrm{C}, \mathrm{C}_{1}\right), 106.7\left(\mathrm{~d},{ }^{2} J_{\mathrm{C}-\mathrm{F}}=7 \mathrm{~Hz}, 1 \mathrm{C}, \mathrm{C}_{5}\right), 55.8\left(\mathrm{~s}, 1 \mathrm{C}, \mathrm{C}_{8}\right), 52.7\left(\mathrm{~d},{ }^{2} J_{\mathrm{C}-\mathrm{F}}\right.$ $\left.=28 \mathrm{~Hz}, 1 \mathrm{C}, \mathrm{C}_{7}\right), 22.3\left(\mathrm{~s}, 3 \mathrm{C}, \mathrm{C}_{9}\right), 18.9\left(\mathrm{~d},{ }^{3} J_{\mathrm{C}-\mathrm{F}}=2 \mathrm{~Hz}, 1 \mathrm{C}, \mathrm{C}_{10}\right) . \mathbf{I R}$ (neat) $v 3198,2979,1690,1494$, 1448, 1390, 1363, 1289, 1171, 1111, 1052, 916, 851, 795, 752, $692 \mathrm{~cm}^{-1}$. HR-MS (ESI+): $\mathrm{m} / \mathrm{z}=$ $270.1330[\mathrm{M}+\mathrm{H}]^{+}$, calcd. for $\mathrm{C}_{14} \mathrm{H}_{21} \mathrm{FNOS}: 270.1328 .[\alpha]_{\mathrm{D}}^{25}=+87\left(c 1.3, \mathrm{CH}_{3} \mathrm{Cl}\right) \cdot R f=0.23\left(20 \% \mathrm{Et}_{2} \mathrm{O}\right.$ in pentane).

$\left(\boldsymbol{R}_{S}\right)-N$-((Z)-2-fluoro-1,3-diphenylallyl)-2-methylpropane-2-sulfinamide (4d-e):

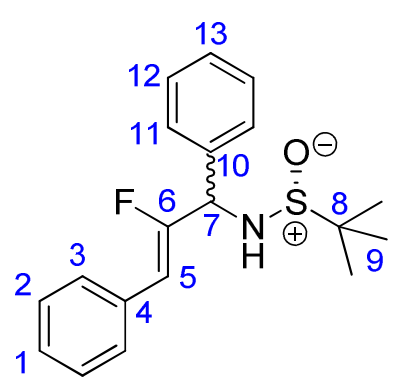

The general procedure for synthesis of the sulfonamides using $(R \mathrm{~s})-\beta$ Fluoroenimine ( 1 eq., $1 \mathrm{~g}, 4.0 \mathrm{mmol}$ ) in toluene $(26 \mathrm{~mL})$ at $-30^{\circ} \mathrm{C}$ during 60 minutes followed by flash chromatography on silica gel (pentane/Et ${ }_{2} \mathrm{O}$ 50/50) afforded $N$-((R,Z)-2-fluoro-1,3-diphenylallyl)-2-methylpropane-2sulfinamide (1.25 g, $3.8 \mathrm{mmol}, 96 \%$ ) as: a white crystalline solid (900 mg, $2.72 \mathrm{mmol}$, yield 69\%) for diastereomer $(S)(R \mathrm{~s})$ and a colorless thick oil (350 $\mathrm{mg}, 1.1 \mathrm{mmol}$, yield $27 \%$ ) for a 50/50 mixture of diastereomers $(R)(R \mathrm{~s})$ and $(S)(R \mathrm{~s})$.

\section{$\underline{(S, R s) \text { diastereoisomer }}(4 d)$ :}

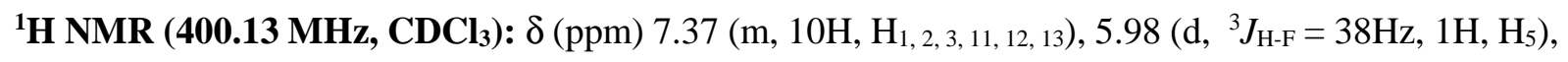
$5.19\left(\mathrm{dd},{ }^{3} J_{\mathrm{H}-\mathrm{F}}=18 \mathrm{~Hz},{ }^{3} J_{\mathrm{H}-\mathrm{H}}=3 \mathrm{~Hz}, 1 \mathrm{H}, \mathrm{H}_{7}\right), 3.56\left(\mathrm{~d},{ }^{3} J_{\mathrm{H}-\mathrm{H}}=3 \mathrm{~Hz}, 1 \mathrm{H}, \mathrm{NH}\right), 1.30\left(\mathrm{~s}, 9 \mathrm{H}, \mathrm{H}_{9}\right) .{ }^{19} \mathbf{F} \mathbf{~ N M R}$ (376.50 MHz, $\left.\mathbf{C D C l}_{3}\right): \delta(\mathrm{ppm})-115.06\left(\mathrm{dd},{ }^{3} J_{\mathrm{F}-\mathrm{H}}=18 \mathrm{~Hz},{ }^{3} J_{\mathrm{F}-\mathrm{H}}=38 \mathrm{~Hz}, 1 \mathrm{~F}, \mathrm{~F}_{6}\right) .{ }^{13} \mathbf{C}$ NMR (75.47 MHz, $\left.\mathbf{C D C l}_{3}\right): \delta(\mathrm{ppm}) 156.9\left(\mathrm{~d},{ }^{1} J_{\mathrm{C}-\mathrm{F}}=270 \mathrm{~Hz}, 1 \mathrm{C}, \mathrm{C}_{6}\right), 138.2\left(\mathrm{~d},{ }^{3} J_{\mathrm{C}-\mathrm{F}}=2 \mathrm{~Hz}, 1 \mathrm{C}, \mathrm{C}_{10}\right), 132.6\left(\mathrm{~d},{ }^{3} J_{\mathrm{C}-\mathrm{F}}=3 \mathrm{~Hz}\right.$, $\left.1 \mathrm{C}, \mathrm{C}_{4}\right), 128.9-127.6\left(10 \mathrm{C}, \mathrm{C}_{1,2,3,11,12,13}\right), 109.3\left(\mathrm{~d},{ }^{2} J_{\mathrm{C}-\mathrm{F}}=6 \mathrm{~Hz}, 1 \mathrm{C}, \mathrm{C}_{5}\right), 60.7\left(\mathrm{~d},{ }^{2} J_{\mathrm{C}-\mathrm{F}}=28 \mathrm{~Hz}, 1 \mathrm{C}, \mathrm{C}_{7}\right)$, $56.1\left(\mathrm{~s}, 1 \mathrm{C}, \mathrm{C}_{8}\right), 22.6$ (s, 3C, $\mathrm{C}_{9}$ ). IR (neat) $v$ 3214, 1682, 1598, 1495, 1449, 1406, 1360, 1255, 1153, 1057, 913, 876, 753, 713, 691, $597 \mathrm{~cm}^{-1}$. HR-MS (ESI+): $\mathrm{m} / \mathrm{z}=332.1498[\mathrm{M}+\mathrm{H}]^{+}$, calcd. for $\mathrm{C}_{19} \mathrm{H}_{23}$ FNOS: 332.1484. Anal. calculated for $\mathbf{C}_{19} \mathbf{H}_{22}$ FNOS: C, 68.85; H, 6.69; N, 4.23; S, 9.67 Found: $\mathrm{C}, 68.84 ; \mathrm{H}, 6.65 ; \mathrm{N}, 4.22 ; \mathrm{S}, 9.75 .[\alpha]_{\mathrm{D}}^{25}=-87\left(c 1.5, \mathrm{CH}_{3} \mathrm{Cl}\right) . R f=0.31$ (40\% $\mathrm{Et}_{2} \mathrm{O}$ in pentane).

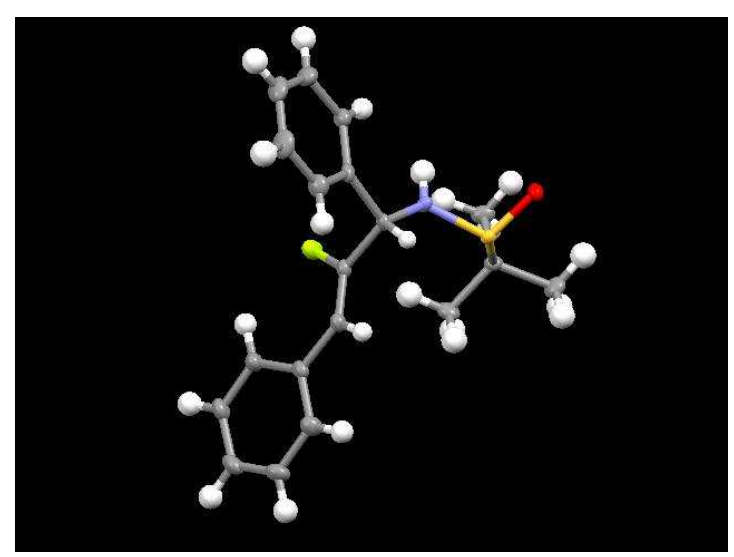




\section{$\underline{(S, R s)+(R, R s) \text { mixture }}(4 d+e)$ :}

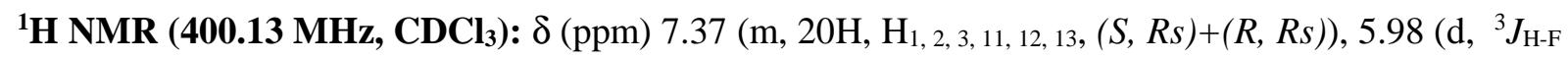
$\left.=38 \mathrm{~Hz}, 1 \mathrm{H}, \mathrm{H}_{5},(S, R s)\right), 5.85\left(\mathrm{~d},{ }^{3} J_{\mathrm{H}-\mathrm{F}}=38 \mathrm{~Hz}, 1 \mathrm{H}, \mathrm{H}_{5},(R, R s)\right), 5.19\left(\mathrm{~m}, 1 \mathrm{H}, \mathrm{H}_{7},(S, R s)\right), 5.16(\mathrm{~m}, 1 \mathrm{H}$, $\left.\mathrm{H}_{7},(R, R s)\right), 3.92\left(\mathrm{~d},{ }^{3} J_{\mathrm{H}-\mathrm{H}}=5 \mathrm{~Hz}, 1 \mathrm{H}, \mathrm{NH},(R, R s)\right), 3.62\left(\mathrm{~d},{ }^{3} J_{\mathrm{H}-\mathrm{H}}=3 \mathrm{~Hz}, 1 \mathrm{H}, \mathrm{NH},(S, R s)\right), 1.28(\mathrm{~s}, 9 \mathrm{H}$, $\left.\mathrm{H}_{9},(S, R s)\right), 1.25\left(\mathrm{~s}, 9 \mathrm{H}, \mathrm{H}_{9},(R, R s)\right) .{ }^{19} \mathbf{F}$ NMR (376.50 $\left.\mathbf{M H z}, \mathbf{C D C l}_{3}\right): \delta(\mathrm{ppm})-113.15\left(\mathrm{dd},{ }^{3} \mathrm{~J}_{\mathrm{F}-\mathrm{H}}=\right.$ $\left.16 \mathrm{~Hz},{ }^{3} J_{\mathrm{F}-\mathrm{H}}=38 \mathrm{~Hz}, 1 \mathrm{~F}, \mathrm{~F}_{6},(R, R s)\right),-115.06\left(\mathrm{dd},{ }^{3} J_{\mathrm{F}-\mathrm{H}}=18 \mathrm{~Hz},{ }^{3} J_{\mathrm{F}-\mathrm{H}}=38 \mathrm{~Hz}, 1 \mathrm{~F}, \mathrm{~F}_{6},(S, R s)\right)$.

(Rs)-N-((Z)-2-fluoro-4-methyl-1-phenylpent-1-en-3-yl)-2-methylpropane-2-sulfinamide (4f-g):

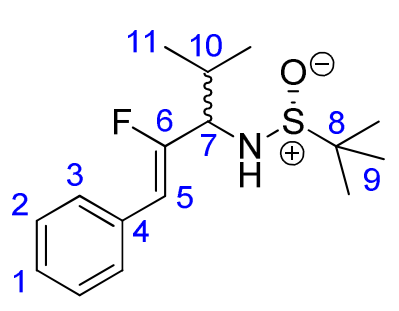

The general procedure for synthesis of the sulfonamides using $(R \mathrm{~s})-\beta$ Fluoroenimine (1 eq., $1 \mathrm{~g}, 4.0 \mathrm{mmol})$ in toluene $(26 \mathrm{~mL})$ followed by flash chromatography on silica gel (pentane/Et $2 \mathrm{O} 70 / 30)$ afforded (Rs)-N-((Z)-2fluoro-4-methyl-1-phenylpent-1-en-3-yl)-2-methylpropane-2-sulfinamide (685 mg, $2.3 \mathrm{mmol}, 59 \%$ ) as: a white crystalline solid (450 mg, $1.5 \mathrm{mmol}$, yield 39\%) for diastereomer $(S)(R \mathrm{~s})$ and a colorless thick oil (225 mg, $0.8 \mathrm{mmol}$, yield $20 \%)$ for diastereomer $(R)(R \mathrm{~s})$.

\section{$\underline{(S, R s) \text { diastereoisomer }(4 f):}$}

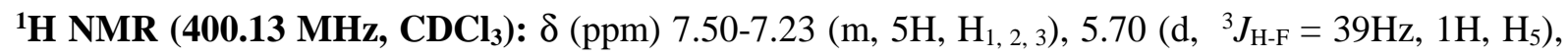
$3.68\left(\mathrm{ddd},{ }^{3} J_{\mathrm{H}-\mathrm{F}}=23 \mathrm{~Hz},{ }^{3} J_{\mathrm{H}-\mathrm{H}}=7 \mathrm{~Hz},{ }^{3} J_{\mathrm{H}-\mathrm{H}}=4 \mathrm{~Hz}, 1 \mathrm{H}, \mathrm{H}_{7}\right), 3.33\left(\mathrm{~d},{ }^{3} J_{\mathrm{H}-\mathrm{H}}=4 \mathrm{~Hz}, 1 \mathrm{H}, \mathrm{NH}\right), 2.05\left(\mathrm{ds},{ }^{3} J_{\mathrm{H}-}\right.$ $\left.\mathrm{H}=7 \mathrm{~Hz},{ }^{3} J_{\mathrm{H}-\mathrm{H}}=7 \mathrm{~Hz}, 1 \mathrm{H}, \mathrm{H}_{10}\right), 1.23\left(\mathrm{~s}, 9 \mathrm{H}, \mathrm{H}_{9}\right), 1.06\left(\mathrm{~m}, 6 \mathrm{H}, \mathrm{H}_{11}\right) .{ }^{19} \mathbf{F}$ NMR (376.50 MHz, CDCl 3$): \delta$ (ppm) $-115.61\left(\mathrm{dd},{ }^{3} J_{\mathrm{F}-\mathrm{H}}=23 \mathrm{~Hz},{ }^{3} J_{\mathrm{F}-\mathrm{H}}=39 \mathrm{~Hz}, 1 \mathrm{~F}, \mathrm{~F}_{6}\right) .{ }^{13} \mathbf{C ~ N M R}\left(\mathbf{7 5 . 4 7} \mathbf{~ M H z}, \mathbf{C D C l}_{3}\right): \delta(\mathrm{ppm}) 157.6$ $\left(\mathrm{d},{ }^{1} J_{\mathrm{C}-\mathrm{F}}=270 \mathrm{~Hz}, 1 \mathrm{C}, \mathrm{C}_{6}\right), 132.9\left(\mathrm{~d},{ }^{3} J_{\mathrm{C}-\mathrm{F}}=3 \mathrm{~Hz}, 1 \mathrm{C}, \mathrm{C}_{4}\right), 128.7\left(\mathrm{~s}, 2 \mathrm{C}, \mathrm{C}_{2}\right), 128.5\left(2 \mathrm{C}, \mathrm{C}_{3}\right), 127.4\left(\mathrm{~d},{ }^{6} J_{\mathrm{C}-}\right.$ $\left.\mathrm{F}=2 \mathrm{~Hz}, 1 \mathrm{C}, \mathrm{C}_{1}\right), 109.3\left(\mathrm{~d},{ }^{2} J_{\mathrm{C}-\mathrm{F}}=7 \mathrm{~Hz}, 1 \mathrm{C}, \mathrm{C}_{5}\right), 63.7\left(\mathrm{~d},{ }^{2} J_{\mathrm{C}-\mathrm{F}}=25 \mathrm{~Hz}, 1 \mathrm{C}, \mathrm{C}_{7}\right), 55.9\left(\mathrm{~s}, 1 \mathrm{C}, \mathrm{C}_{8}\right), 31.5(\mathrm{~s}$, $1 \mathrm{C}, \mathrm{C}_{10}$ ), 22.5 (s, 3C, $\mathrm{C}_{9}$ ), 19.3 (s, 2C, $\mathrm{C}_{11}$ ). IR (neat) v 3205, 2961, 2870, 1681, 1491, 1466, 1415, 1388, 1361, 1322, 1280, 1163, 1036, 941, 919, 845, 762, 734, 695, 665, $600 \mathrm{~cm}^{-1}$. HR-MS (ESI+): $\mathrm{m} / \mathrm{z}=$ 298.1644 [M+H] $]^{+}$, calcd. for $\mathrm{C}_{16} \mathrm{H}_{25}$ FNOS: 298.1641. Anal. calculated for $\mathbf{C}_{16} \mathbf{H}_{24}$ FNOS: C, 64.61; H, 8.13; N, 4.71; S, 10.78 Found: C, 64.72; H, 8.13; N, 4.74; S, 10.78. $[\alpha]_{\mathrm{D}}^{25}=+15\left(c 2.0, \mathrm{CH}_{3} \mathrm{Cl}\right) . R f=$ $0.30\left(50 \% \mathrm{Et}_{2} \mathrm{O}\right.$ in pentane). 


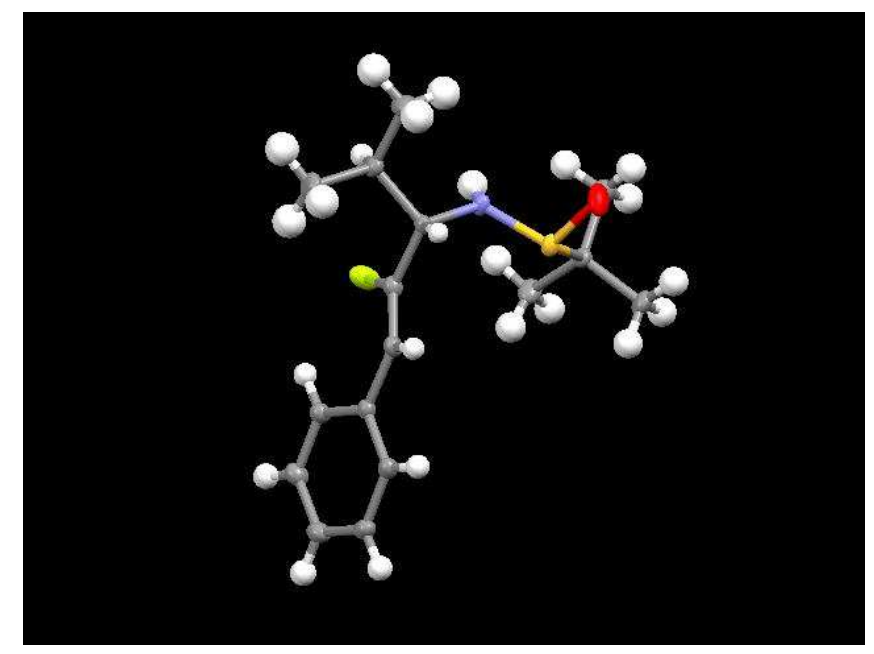

CCDC number: 1482134

\section{$\underline{(R, R s) \text { diastereoisomer }(4 g) \text { : }}$}

${ }^{1} \mathbf{H}$ NMR (400.13 MHz, $\left.\mathbf{C D C l}_{3}\right): \delta(\mathrm{ppm}) 7.51-7.20\left(\mathrm{~m}, 5 \mathrm{H}, \mathrm{H}_{1,2,3}\right), 5.77\left(\mathrm{~d},{ }^{3} J_{\mathrm{H}-\mathrm{F}}=39 \mathrm{~Hz}, 1 \mathrm{H}, \mathrm{H}_{5}\right)$, $3.52\left(\mathrm{~m}, 1 \mathrm{H}, \mathrm{H}_{7}\right), 3.52$ (br., $\left.1 \mathrm{H}, \mathrm{NH}\right), 2.04\left(\mathrm{ds},{ }^{3} J_{\mathrm{H}-\mathrm{H}}=7 \mathrm{~Hz},{ }^{3} J_{\mathrm{H}-\mathrm{H}}=7 \mathrm{~Hz}, 1 \mathrm{H}, \mathrm{H}_{10}\right), 1.25\left(\mathrm{~s}, 9 \mathrm{H}, \mathrm{H}_{9}\right), 1.02$ $\left(\mathrm{m}, 6 \mathrm{H}, \mathrm{H}_{11}\right) .{ }^{19} \mathbf{F}$ NMR (376.50 MHz, $\left.\mathbf{C D C l}_{3}\right): \delta(\mathrm{ppm})-116.75\left(\mathrm{dd},{ }^{3} \mathrm{~J}_{\mathrm{F}-\mathrm{H}}=22 \mathrm{~Hz},{ }^{3} \mathrm{~J}_{\mathrm{F}-\mathrm{H}}=40 \mathrm{~Hz}, 1 \mathrm{~F}\right.$, $\left.\mathrm{F}_{6}\right) .{ }^{13} \mathrm{C}$ NMR (75.47 MHz, $\left.\mathbf{C D C l}_{3}\right): \delta(\mathrm{ppm}) 158.2\left(\mathrm{~d},{ }^{1} J_{\mathrm{C}-\mathrm{F}}=270 \mathrm{~Hz}, 1 \mathrm{C}, \mathrm{C}_{6}\right), 132.6\left(\mathrm{~d},{ }^{3} J_{\mathrm{C}-\mathrm{F}}=2 \mathrm{~Hz}\right.$, $\left.1 \mathrm{C}, \mathrm{C}_{4}\right), 128.7\left(\mathrm{~s}, 2 \mathrm{C}, \mathrm{C}_{2}\right), 128.2\left(2 \mathrm{C}, \mathrm{C}_{3}\right), 127.1\left(\mathrm{~d},{ }^{6} J_{\mathrm{C}-\mathrm{F}}=2 \mathrm{~Hz}, 1 \mathrm{C}, \mathrm{C}_{1}\right), 108.3\left(\mathrm{~d},{ }^{2} J_{\mathrm{C}-\mathrm{F}}=7 \mathrm{~Hz}, 1 \mathrm{C}, \mathrm{C}_{5}\right)$, $64.7\left(\mathrm{~d},{ }^{2} J_{\mathrm{C}-\mathrm{F}}=26 \mathrm{~Hz}, 1 \mathrm{C}, \mathrm{C}_{7}\right), 56.4\left(\mathrm{~s}, 1 \mathrm{C}, \mathrm{C}_{8}\right), 31.3\left(\mathrm{~s}, 1 \mathrm{C}, \mathrm{C}_{10}\right), 22.5\left(\mathrm{~s}, 3 \mathrm{C}, \mathrm{C}_{9}\right), 19.3\left(\mathrm{~s}, 1 \mathrm{C}, \mathrm{C}_{11}\right), 19.0$ $\left(\mathrm{s}, 1 \mathrm{C}, \mathrm{C}_{11}\right.$ ). IR (neat) v 3203, 2960, 2868, 1687, 1467, 1388, 1363, 1167, 1118, 1054, 876, 754, 693, $594 \mathrm{~cm}^{-1}$. HR-MS (ESI+): $\mathrm{m} / \mathrm{z}=298.1639[\mathrm{M}+\mathrm{H}]^{+}$, calcd. for $\mathrm{C}_{16} \mathrm{H}_{25} \mathrm{FNOS}$ : 298.1641. Anal. calculated for $\mathbf{C}_{16} \mathbf{H}_{24}$ FNOS: C, 64.61; H, 8.13; N, 4.71; S, 10.78 Found: C, 64.72; H, 8.13; N, 4.74; S, 10.78. $[\alpha]_{\mathrm{D}}^{25}=-14.5\left(c 4.0, \mathrm{CH}_{3} \mathrm{Cl}\right) . R f=0.32\left(50 \% \mathrm{Et}_{2} \mathrm{O}\right.$ in pentane).

(Z)-N-(3-fluoro-1,4-diphenylbut-3-en-2-yl)-2-methylpropane-2-sulfinamide (4h):<smiles>CC(C)(C)S(=O)N[C@@H](Cc1ccccc1)/C(F)=C/c1ccccc1</smiles>

The general procedure for synthesis of the sulfinamides using a racemic $\beta$ Fluoroenimine (1 eq., $400 \mathrm{mg}, 1.6 \mathrm{mmol})$ in toluene $(15 \mathrm{~mL})$ followed by flash chromatography on silica gel (pentane/Et $2 \mathrm{O} 50 / 50$ to 20/80) afforded (Z)-N-(3fluoro-1,4-diphenylbut-3-en-2-yl)-2-methylpropane-2-sulfinamide as: a colorless thick oil (340 mg, $1.0 \mathrm{mmol}$, yield $63 \%$ ).

\section{diastereoisomeric mixture (4h):}


${ }^{1} \mathbf{H}$ NMR (400.13 MHz, $\left.\mathbf{C D C l}_{3}\right): \delta(\mathrm{ppm}) 7.46\left(\mathrm{~d},{ }^{3} \mathrm{~J}_{\mathrm{H}-\mathrm{H}}=7 \mathrm{~Hz}, 2 \mathrm{H}, \mathrm{H}_{3}\right), 7.28\left(\mathrm{~m}, 8 \mathrm{H}, \mathrm{H}_{1,2,12,13,14}\right), 5.64$ $\left(\mathrm{d},{ }^{3} J_{\mathrm{H}-\mathrm{F}}=39 \mathrm{~Hz}, 1 \mathrm{H}, \mathrm{H}_{5}\right), 4.27\left(\mathrm{ddt},{ }^{3} J_{\mathrm{H}-\mathrm{F}}=19 \mathrm{~Hz},{ }^{3} J_{\mathrm{H}-\mathrm{H}}=7 \mathrm{~Hz},{ }^{3} J_{\mathrm{H}-\mathrm{H}}=4 \mathrm{~Hz}, 1 \mathrm{H}, \mathrm{H}_{7}\right), 3.38\left(\mathrm{~d},{ }^{3} J_{\mathrm{H}-\mathrm{H}}=\right.$ $4 \mathrm{~Hz}, 1 \mathrm{H}, \mathrm{NH}), 3.16$ (m, 2H, H10), 1.20 (s, 9H, H9). ${ }^{19} \mathbf{F}$ NMR (376.50 MHz, CDCl 3 ): $\delta(\mathrm{ppm})-118.14$ $\left(\mathrm{dd},{ }^{3} J_{\mathrm{F}-\mathrm{H}}=19 \mathrm{~Hz},{ }^{3} J_{\mathrm{F}-\mathrm{H}}=40 \mathrm{~Hz}, 1 \mathrm{~F}, \mathrm{~F}_{6}\right) .{ }^{13} \mathbf{C} \mathbf{N M R}\left(\mathbf{7 5 . 4 7} \mathbf{M H z}, \mathbf{C D C l}_{3}\right): \delta(\mathrm{ppm}) 156.7\left(\mathrm{~d},{ }^{1} J_{\mathrm{C}-\mathrm{F}}=\right.$ $\left.270 \mathrm{~Hz}, 1 \mathrm{C}, \mathrm{C}_{6}\right), 135.7$ (s, 1C, $\left.\mathrm{C}_{11}\right), 132.6\left(\mathrm{~d},{ }^{3} J_{\mathrm{C}-\mathrm{F}}=2 \mathrm{~Hz}, 1 \mathrm{C}, \mathrm{C}_{4}\right), 129.6$ (s, 2C, $\left.\mathrm{C}_{2}\right), 128.8\left(\mathrm{~s}, 2 \mathrm{C}, \mathrm{C}_{13}\right)$, $128.7\left(\mathrm{~d},{ }^{4} J_{\mathrm{C}-\mathrm{F}}=7 \mathrm{~Hz}, 2 \mathrm{C}, \mathrm{C}_{3}\right), 128.5\left(2 \mathrm{C}, \mathrm{C}_{12}\right), 127.5\left(\mathrm{~d},{ }^{6} J_{\mathrm{C}-\mathrm{F}}=2 \mathrm{~Hz}, 1 \mathrm{C}, \mathrm{C}_{1}\right), 127.2\left(\mathrm{~s}, 1 \mathrm{C}, \mathrm{C}_{14}\right), 109.4$ $\left(\mathrm{d},{ }^{2} J_{\mathrm{C}-\mathrm{F}}=6 \mathrm{~Hz}, 1 \mathrm{C}, \mathrm{C}_{5}\right), 58.1\left(\mathrm{~d},{ }^{2} J_{\mathrm{C}-\mathrm{F}}=27 \mathrm{~Hz}, 1 \mathrm{C}, \mathrm{C}_{7}\right), 55.9\left(\mathrm{~s}, 1 \mathrm{C}, \mathrm{C}_{8}\right), 39.7\left(\mathrm{~s}, 1 \mathrm{C}, \mathrm{C}_{10}\right), 22.5(\mathrm{~s}, 3 \mathrm{C}$, $\mathrm{C}_{9}$ ). IR (neat) $v$ 3180, 3026, 2957, 2873, 1688, 1602, 1495, 1473, 1455, 1363, 1302, 1160, 1050, 947, 914, 882, 847, 748, 712, $692 \mathrm{~cm}^{-1}$. HR-MS (ESI+): $\mathrm{m} / \mathrm{z}=346.1648[\mathrm{M}+\mathrm{H}]^{+}$, calcd. for $\mathrm{C}_{20} \mathrm{H}_{25} \mathrm{FNOS}$ : 346.1641. $R f=0.22$ (like) $\left(30 \% \mathrm{Et}_{2} \mathrm{O}\right.$ in pentane). $R f=0.26$ (unlike) $\left(30 \% \mathrm{Et}_{2} \mathrm{O}\right.$ in pentane).

c. Synthesis of the bisolefins bearing secondary amide 9

\section{General procedure:}

The starting sulfinamide (1eq.) was placed in a round-bottom flask equipped with a stir bar under argon atmosphere, then dissolved in methanol. The mixture was cooled down to $0^{\circ} \mathrm{C}$ and trimethylsillyl chloride (4 eq.) was slowly added. The mixture was then placed at room temperature and stirred for 1 hour. The solution was then concentrated under reduced pressure to afford the desired primary amine chlorhydrate salt which was directly involved in the further step. The starting primary amine (1eq.) was placed in a round-bottom flask, equipped with a stir bar under argon atmosphere, and dissolved in dichloromethane. Triethylamine (4 eq.) was added and the mixture was stirred for a few minutes. Then,

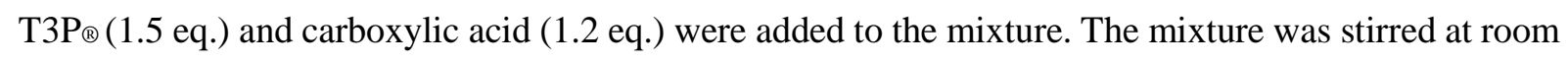
temperature and the progress of the reaction monitored through ${ }_{19} \mathrm{~F}$ NMR and TLC. After completion, the reaction was stopped by adding water followed by extraction of the aqueous phase with dichloromethane (3x). The organic layers were combined and dried over $\mathrm{MgSO}_{4}$. After removal of the $\mathrm{MgSO}_{4}$ and evaporation of the volatile materials under reduced pressure, the crude was purified by flash chromatography on silica gel (Pentane:Et $2 \mathrm{O}$ ) to afford the expected diene.

(R,Z)-N-(3-fluoro-4-phenylbut-3-en-2-yl)but-3-enamide (9c):

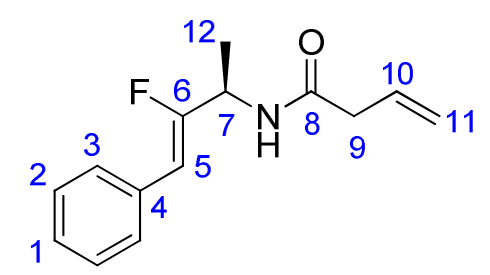

The general two steps procedure for the synthesis of bisolefins bearing secondary amide using sulfinamide $4 \mathbf{c}$ ( 1 eq., $150 \mathrm{mg}, 744 \mu \mathrm{mol})$ afforded the (R,Z)-N-(3-fluoro-4-phenylbut-3-en-2-yl)but-3enamide (168 mg, $720 \mu \mathrm{mol}$, yield $97 \%$ ) as a light yellow oil. 
${ }^{1} \mathbf{H}$ NMR (400.13 MHz, CDCl 3$): \delta(\mathrm{ppm}) 7.49-7.23\left(\mathrm{~m}, 5 \mathrm{H}, \mathrm{H}_{1,2,3}\right), 5.95\left(\mathrm{ddt},{ }^{3} J_{\mathrm{H}-\mathrm{H}}=17 \mathrm{~Hz},{ }^{3} J_{\mathrm{H}-\mathrm{H}}=\right.$ $\left.10 \mathrm{~Hz},{ }^{3} J_{\mathrm{H}-\mathrm{H}}=7 \mathrm{~Hz}, 1 \mathrm{H}, \mathrm{H}_{10}\right), 5.81\left(\mathrm{~d},{ }^{3} J_{\mathrm{H}-\mathrm{H}}=9 \mathrm{~Hz}, 1 \mathrm{H}, \mathrm{NH}\right), 5.72\left(\mathrm{~d},{ }^{3} J_{\mathrm{H}-\mathrm{F}}=40 \mathrm{~Hz}, 1 \mathrm{H}, \mathrm{H}_{5}\right), 5.26(\mathrm{~m}, 2 \mathrm{H}$, $\left.\mathrm{H}_{11}\right), 4.84\left(\mathrm{ddq},{ }^{3} J_{\mathrm{H}-\mathrm{F}}=20 \mathrm{~Hz},{ }^{3} J_{\mathrm{H}-\mathrm{H}}=9 \mathrm{~Hz},{ }^{3} J_{\mathrm{H}-\mathrm{H}}=7 \mathrm{~Hz}, 1 \mathrm{H}, \mathrm{H}_{7}\right), 3.05\left(\mathrm{~d},{ }^{3} J_{\mathrm{H}-\mathrm{H}}=7 \mathrm{~Hz}, 2 \mathrm{H}, \mathrm{H}_{9}\right), 1.41(\mathrm{~d}$, $\left.{ }^{3} J_{\mathrm{H}-\mathrm{H}}=7 \mathrm{~Hz}, 3 \mathrm{H}, \mathrm{H}_{12}\right) .{ }^{19} \mathbf{F}$ NMR (376.50 MHz, $\left.\mathbf{C D C l}_{3}\right): \delta(\mathrm{ppm})-117.65\left(\mathrm{dd},{ }^{3} J_{\mathrm{F}-\mathrm{H}}=19 \mathrm{~Hz},{ }^{3} J_{\mathrm{F}-\mathrm{H}}=\right.$ $\left.40 \mathrm{~Hz}, 1 \mathrm{~F}, \mathrm{~F}_{6}\right) \cdot{ }^{13} \mathbf{C}$ NMR (75.47 MHz, $\left.\mathbf{C D C l}_{3}\right): \delta(\mathrm{ppm}) 169.6\left(\mathrm{~s}, 1 \mathrm{C}, \mathrm{C}_{8}\right), 158.7\left(\mathrm{~d},{ }^{1} J_{\mathrm{C}-\mathrm{F}}=269 \mathrm{~Hz}, 1 \mathrm{C}\right.$, $\left.\mathrm{C}_{6}\right), 132.7\left(\mathrm{~d},{ }^{3} J_{\mathrm{C}-\mathrm{F}}=2 \mathrm{~Hz}, 1 \mathrm{C}, \mathrm{C}_{1}\right), 131.0\left(\mathrm{~s}, 1 \mathrm{C}, \mathrm{C}_{10}\right), 128.7\left(2 \mathrm{C}, \mathrm{C}_{2}\right), 128.5\left(2 \mathrm{C}, \mathrm{C}_{3}\right), 127.5\left(\mathrm{~d},{ }^{6} J_{\mathrm{C}-\mathrm{F}}=\right.$ $\left.2 \mathrm{~Hz}, 1 \mathrm{C}, \mathrm{C}_{1}\right), 120.2\left(\mathrm{~s}, 1 \mathrm{C}, \mathrm{C}_{10}\right), 108.5\left(\mathrm{~d},{ }^{2} J_{\mathrm{C}-\mathrm{F}}=7 \mathrm{~Hz}, 1 \mathrm{C}, \mathrm{C}_{5}\right), 46.9\left(\mathrm{~d},{ }^{2} J_{\mathrm{C}-\mathrm{F}}=28 \mathrm{~Hz}, 1 \mathrm{C}, \mathrm{C}_{7}\right), 41.6(\mathrm{~s}$, $\left.1 \mathrm{C}, \mathrm{C}_{9}\right), 18.5\left(\mathrm{~d},{ }^{3} J_{\mathrm{C}-\mathrm{F}}=2 \mathrm{~Hz}, 1 \mathrm{C}, \mathrm{C}_{12}\right)$. IR (neat) $v 3278,3060,2922,1685,1641,1541,1492,1446$, 1403, 1346, 1234, 1166, 1099, 1036, 991, 914, 859, 830, 749, 689, $590 \mathrm{~cm}^{-1}$. HR-MS (ESI+): m/z = 234.1295 $[\mathrm{M}+\mathrm{H}]^{+}$, calcd. for $\mathrm{C}_{14} \mathrm{H}_{17} \mathrm{FNO}$ : 234.1294. Anal. calculated for $\mathbf{C}_{\mathbf{1 4}} \mathbf{H}_{16} \mathbf{F N O}$ : C, 72.08; $\mathrm{H}$, $6.91 ; \mathrm{N}, 6.00$; Found: C, 72.25; H, 7.71; N, 6.19. $R f=0.20\left(50 \% \mathrm{Et}_{2} \mathrm{O}\right.$ in pentane.

(R,Z)-N-(2-fluoro-1,3-diphenylallyl)but-3-enamide (9e):

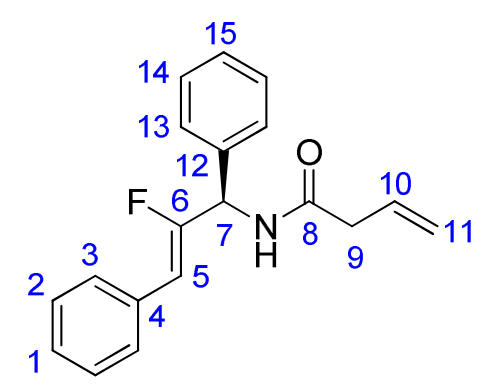

The general two steps procedure for the synthesis of bisolefins bearing secondary amide using sulfinamide 4e (1 eq., $260 \mathrm{mg}, 986 \mu \mathrm{mol})$ afforded the (R,Z)-N-(2-fluoro-1,3-diphenylallyl)but-3-enamide (239 mg, $809 \mu \mathrm{mol}$, yield $83 \%$ ) as a colorless oil.

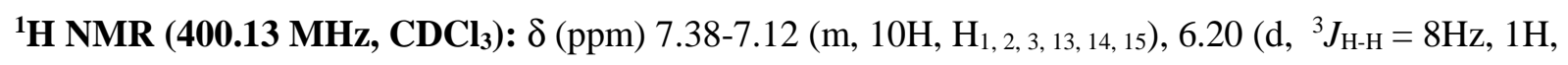
$\mathrm{NH}), 5.86\left(\mathrm{ddt},{ }^{3} J_{\mathrm{H}-\mathrm{H}}=17 \mathrm{~Hz},{ }^{3} J_{\mathrm{H}-\mathrm{H}}=10 \mathrm{~Hz},{ }^{3} J_{\mathrm{H}-\mathrm{H}}=7 \mathrm{~Hz}, 1 \mathrm{H}, \mathrm{H}_{10}\right), 5.79\left(\mathrm{dd},{ }^{3} J_{\mathrm{H}-\mathrm{F}}=20 \mathrm{~Hz},{ }^{3} J_{\mathrm{H}-\mathrm{H}}=8 \mathrm{~Hz}\right.$, $\left.1 \mathrm{H}, \mathrm{H}_{7}\right), 5.71\left(\mathrm{~d},{ }^{3} J_{\mathrm{H}-\mathrm{F}}=39 \mathrm{~Hz}, 1 \mathrm{H}, \mathrm{H}_{5}\right), 5.16\left(\mathrm{~m}, 2 \mathrm{H}, \mathrm{H}_{11}\right), 3.00\left(\mathrm{~d},{ }^{3} J_{\mathrm{H}-\mathrm{H}}=7 \mathrm{~Hz}, 2 \mathrm{H}, \mathrm{H}_{9}\right) .{ }^{19} \mathbf{F} \mathbf{~ N M R}$ (376.50 MHz, $\left.\mathbf{C D C l}_{3}\right): \delta(\mathrm{ppm})-114.73\left(\mathrm{dd},{ }^{3} \mathrm{~J}_{\mathrm{F}-\mathrm{H}}=20 \mathrm{~Hz},{ }^{3} J_{\mathrm{F}-\mathrm{H}}=39 \mathrm{~Hz}, 1 \mathrm{~F}, \mathrm{~F}_{6}\right) .{ }^{13} \mathbf{C}$ NMR (75.47 MHz, $\left.\mathbf{C D C l}_{3}\right): \delta(\mathrm{ppm}) 169.72\left(\mathrm{~s}, 1 \mathrm{C}, \mathrm{C}_{8}\right), 156.8\left(\mathrm{~d},{ }^{1} J_{\mathrm{C}-\mathrm{F}}=269 \mathrm{~Hz}, 1 \mathrm{C}, \mathrm{C}_{6}\right), 137.6\left(\mathrm{~s}, 1 \mathrm{C}, \mathrm{C}_{12}\right), 132.5\left(\mathrm{~d},{ }^{3} J_{\mathrm{C}-\mathrm{F}}\right.$ $\left.=2 \mathrm{~Hz}, 1 \mathrm{C}, \mathrm{C}_{4}\right), 130.9\left(\mathrm{~s}, 1 \mathrm{C}, \mathrm{C}_{10}\right), 128.9-128.2\left(8 \mathrm{C}, \mathrm{C}_{2,3}, 13,14\right), 127.7\left(\mathrm{~d},{ }^{6} J_{\mathrm{C}-\mathrm{F}}=2 \mathrm{~Hz}, 1 \mathrm{C}, \mathrm{C}_{1}\right), 126.9(\mathrm{~s}$, $\left.1 \mathrm{C}, \mathrm{C}_{15}\right), 120.4\left(\mathrm{~s}, 1 \mathrm{C}, \mathrm{C}_{11}\right), 108.7\left(\mathrm{~d},{ }^{2} J_{\mathrm{C}-\mathrm{F}}=7 \mathrm{~Hz}, 1 \mathrm{C}, \mathrm{C}_{5}\right), 54.7\left(\mathrm{~d},{ }^{2} J_{\mathrm{C}-\mathrm{F}}=28 \mathrm{~Hz}, 1 \mathrm{C}, \mathrm{C}_{7}\right), 41.6(\mathrm{~s}, 1 \mathrm{C}$, $\mathrm{C}_{9}$ ). IR (neat) $v$ 3291, 3030, 1691, 1647, 1527, 1494, 1449, 1404, 1359, 1265, 1214, 1154, 1083, 987 , 922, 898, 935, 753, $692 \mathrm{~cm}^{-1}$. HR-MS (ESI+): $\mathrm{m} / \mathrm{z}=296.1452[\mathrm{M}+\mathrm{H}]^{+}$, calcd. for $\mathrm{C}_{19} \mathrm{H}_{19} \mathrm{FNO}$ : 296.1451. Anal. calculated for $\mathbf{C}_{19} \mathbf{H}_{18} \mathbf{F N O}$ : $\mathrm{C}, 77.27$; H, 6.14; N, 4.74; S, 9.67 Found: C, 77.25; H, $6.94 ; \mathrm{N}, 5.01 . R f=0.30\left(40 \% \mathrm{Et}_{2} \mathrm{O}\right.$ in pentane).

(R,Z)-N-(2-fluoro-4-methyl-1-phenylpent-1-en-3-yl)but-3-enamide (9g): 


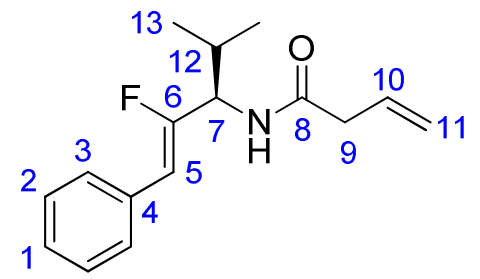

The general two steps procedure for the synthesis of bisolefins bearing secondary amide using sulfinamide $\mathbf{4 g}$ (1 eq., $100 \mathrm{mg}, 435 \mu \mathrm{mol}$ ) afforded the (R,Z)-N-(2-fluoro-4-methyl-1-phenylpent-1-en-3yl)but-3-enamide (85 mg, $330 \mu \mathrm{mol}$, yield $75 \%$ ) as a light yellow oil.

${ }^{1} \mathbf{H}$ NMR (400.13 MHz, $\left.\mathbf{C D C l}_{3}\right): \delta(\mathrm{ppm}) 7.49-7.22\left(\mathrm{~m}, 5 \mathrm{H}, \mathrm{H}_{1,2,3}\right), 5.97\left(\mathrm{ddt},{ }^{3} J_{\mathrm{H}-\mathrm{H}}=17 \mathrm{~Hz},{ }^{3} J_{\mathrm{H}-\mathrm{H}}=\right.$ $\left.10 \mathrm{~Hz},{ }^{3} J_{\mathrm{H}-\mathrm{H}}=7 \mathrm{~Hz}, 1 \mathrm{H}, \mathrm{H}_{10}\right), 5.88\left(\mathrm{~d},{ }^{3} J_{\mathrm{H}-\mathrm{H}}=10 \mathrm{~Hz}, 1 \mathrm{H}, \mathrm{NH}\right), 5.71\left(\mathrm{~d},{ }^{3} J_{\mathrm{H}-\mathrm{F}}=40 \mathrm{~Hz}, 1 \mathrm{H}, \mathrm{H}_{5}\right), 5.28(\mathrm{~m}$, $\left.2 \mathrm{H}, \mathrm{H}_{11}\right), 4.41\left(\mathrm{ddd},{ }^{3} J_{\mathrm{H}-\mathrm{F}}=25 \mathrm{~Hz},{ }^{3} J_{\mathrm{H}-\mathrm{H}}=9 \mathrm{~Hz},{ }^{3} J_{\mathrm{H}-\mathrm{H}}=9 \mathrm{~Hz}, 1 \mathrm{H}, \mathrm{H}_{7}\right), 3.07\left(\mathrm{~d},{ }^{3} J_{\mathrm{H}-\mathrm{H}}=7 \mathrm{~Hz}, 2 \mathrm{H}, \mathrm{H}_{9}\right), 2.00$ $\left(\mathrm{ds},{ }^{3} J_{\mathrm{H}-\mathrm{H}}=7 \mathrm{~Hz},{ }^{3} J_{\mathrm{H}-\mathrm{H}}=1 \mathrm{~Hz}, 1 \mathrm{H}, \mathrm{H}_{12}\right), 1.01\left(\mathrm{~m}, 6 \mathrm{H}, \mathrm{H}_{13}\right) .{ }^{19} \mathbf{F}$ NMR (376.50 MHz, $\left.\mathbf{C D C l}_{3}\right): \delta(\mathrm{ppm})-$ $118.15\left(\mathrm{dd},{ }^{3} J_{\mathrm{F}-\mathrm{H}}=25 \mathrm{~Hz},{ }^{3} J_{\mathrm{F}-\mathrm{H}}=39 \mathrm{~Hz}, 1 \mathrm{~F}, \mathrm{~F}_{6}\right) .{ }^{13} \mathbf{C} \mathbf{N M R}\left(\mathbf{7 5 . 4 7} \mathbf{~ M H z}, \mathbf{C D C l}_{3}\right): \delta(\mathrm{ppm}) 169.8(\mathrm{~s}, 1 \mathrm{C}$, $\left.\mathrm{C}_{8}\right), 157.2\left(\mathrm{~d},{ }^{1} J_{\mathrm{C}-\mathrm{F}}=269 \mathrm{~Hz}, 1 \mathrm{C}, \mathrm{C}_{6}\right), 132.8\left(\mathrm{~d},{ }^{3} J_{\mathrm{C}-\mathrm{F}}=3 \mathrm{~Hz}, 1 \mathrm{C}, \mathrm{C}_{4}\right), 131.2\left(\mathrm{~s}, 1 \mathrm{C}, \mathrm{C}_{10}\right), 128.7\left(2 \mathrm{C}, \mathrm{C}_{2}\right)$, $128.5\left(2 \mathrm{C}, \mathrm{C}_{3}\right), 127.4\left(\mathrm{~d},{ }^{6} J_{\mathrm{C}-\mathrm{F}}=2 \mathrm{~Hz}, 1 \mathrm{C}, \mathrm{C}_{1}\right), 120.2\left(\mathrm{~s}, 1 \mathrm{C}, \mathrm{C}_{11}\right), 108.3\left(\mathrm{~d},{ }^{2} J_{\mathrm{C}-\mathrm{F}}=7 \mathrm{~Hz}, 1 \mathrm{C}, \mathrm{C}_{5}\right), 57.1(\mathrm{~d}$, $\left.{ }^{2} J_{\mathrm{C}-\mathrm{F}}=26 \mathrm{~Hz}, 1 \mathrm{C}, \mathrm{C}_{7}\right), 41.7\left(\mathrm{~s}, 1 \mathrm{C}, \mathrm{C}_{9}\right), 30.5\left(1 \mathrm{C}, \mathrm{C}_{12}\right), 19.5$ (s, 1C, $\left.\mathrm{C}_{13}\right), 18.9$ (s, 1C, $\left.\mathrm{C}_{13}\right)$. IR (neat) $v$ 3331, 2963, 1639, 1531, 1353, 1258, 1163, 1074, 996, 912, 840, 762, 693, $595 \mathrm{~cm}^{-1}$. HR-MS (ESI+): $\mathrm{m} / \mathrm{z}=262.1620[\mathrm{M}+\mathrm{H}]^{+}$, calcd. for $\mathrm{C}_{16} \mathrm{H}_{21} \mathrm{FNO}: 262.1607$. Anal. calculated for $\mathrm{C}_{16} \mathrm{H}_{20} \mathrm{FNO}: \mathrm{C}, 73.53$; $\mathrm{H}, 7.71$; N, 5.36; Found: C, 73.53; H, 7.75; N, 5.42. $R f=0.33$ (40\% $\mathrm{Et}_{2} \mathrm{O}$ in pentane).

\section{d. Synthesis of the secondary PMB-amines 7}

\section{General procedure:}

The starting sulfinamide (1eq.) was placed in a round-bottom flask equipped with a stir bar under argon atmosphere and dissolved in methanol. The mixture was cooled down to $0^{\circ} \mathrm{C}$ and trimethylsillyl chloride (4 eq.) was slowly added. The mixture was then placed at room temperature and stirred for 1 hour. The solution was then concentrated under reduced pressure to afford the desired primary amine chlorhydrate salt which was directly involved in the further step. The Starting Amine (1eq.) was placed in a two-neck flask, equipped with a reflux condenser and a stir bar under argon atmosphere, and dissolved in acetonitrile. 4-methoxybenzyl chloride (1.2 eq.) and potassium carbonate (5 eq.) were then added in this order. The mixture was heated to $60^{\circ} \mathrm{C}$ and progress of the reaction monitored through ${ }^{19} \mathrm{~F}$ NMR and TLC. After completion, the reaction was stopped by adding water followed by extraction of the aqueous phase with dichloromethane (3x). The organic layers were combined and dried over $\mathrm{MgSO}_{4}$. After removal of the $\mathrm{MgSO}_{4}$ and evaporation of the volatile materials under reduced pressure, the crude was purified by flash chromatography on silica gel (Pentane:Et $\left.{ }_{2} \mathrm{O}\right)$ to afford the expected $\mathrm{PMB}$ protected amine.

(S,Z)-3-fluoro-N-(4-methoxybenzyl)-4-phenylbut-3-en-2-amine (7b): 


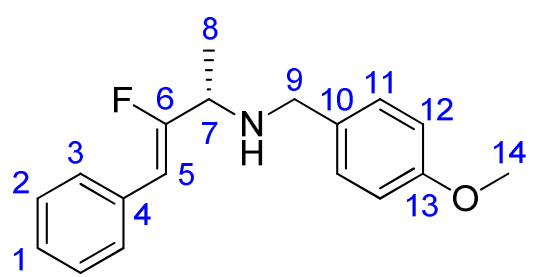

The general two steps procedure for the synthesis of secondary PMB amines using sulfinamide $4 \mathbf{b}$ (1 eq., $230 \mathrm{mg}, 854 \mu \mathrm{mol}$ ) afforded the (S,Z)-3-fluoro-N-(4-methoxybenzyl)-4-phenylbut-3en-2-amine (155 mg, $543 \mu \mathrm{mol}$, yield $64 \%$ ) as a colorless oil.

${ }^{1} \mathbf{H}$ NMR (400.13 MHz, CDCl $)$ : $\delta(\mathrm{ppm}) 7.44\left(\mathrm{~d},{ }^{3} J_{\mathrm{H}-\mathrm{H}}=9 \mathrm{~Hz}, 2 \mathrm{H}, \mathrm{H}_{3}\right), 7.28-7.14\left(\mathrm{~m}, 5 \mathrm{H}, \mathrm{H}_{1,2,11}\right), 6.78$ $\left(\mathrm{d},{ }^{3} J_{\mathrm{H}-\mathrm{H}}=9 \mathrm{~Hz}, 2 \mathrm{H}, \mathrm{H}_{12}\right), 5.59\left(\mathrm{~d},{ }^{3} J_{\mathrm{H}-\mathrm{F}}=39 \mathrm{~Hz}, 1 \mathrm{H}, \mathrm{H}_{5}\right), 3.70\left(\mathrm{~s}, 3 \mathrm{H}, \mathrm{H}_{14}\right), 3.77\left(\mathrm{~d},{ }^{3} J_{\mathrm{H}-\mathrm{H}}=13 \mathrm{~Hz}, 1 \mathrm{H}\right.$, $\left.\mathrm{H}_{9}\right), 3.62\left(\mathrm{~d},{ }^{3} J_{\mathrm{H}-\mathrm{H}}=13 \mathrm{~Hz}, 1 \mathrm{H}, \mathrm{H}_{9}\right), 3.33\left(\mathrm{dquad},{ }^{3} J_{\mathrm{H}-\mathrm{H}}=7 \mathrm{~Hz},{ }^{3} J_{\mathrm{H}-\mathrm{F}}=22 \mathrm{~Hz}, 1 \mathrm{H}, \mathrm{H}_{7}\right), 3.16($ br. s, $1 \mathrm{H}$, NH), $1.31\left(\mathrm{~d},{ }^{3} J_{\mathrm{H}-\mathrm{H}}=7 \mathrm{~Hz}, 3 \mathrm{H}, \mathrm{H}_{8}\right) .{ }^{19} \mathbf{F}$ NMR (376.50 $\left.\mathbf{~ M H z}, \mathbf{C D C l}_{3}\right): \delta(\mathrm{ppm})-118.43\left(\mathrm{dd},{ }^{3} J_{\mathrm{F}-\mathrm{H}}=22 \mathrm{~Hz}\right.$, $\left.{ }^{3} J_{\mathrm{F}-\mathrm{H}}=39 \mathrm{~Hz}, 1 \mathrm{~F}, \mathrm{~F}_{6}\right) .{ }^{13} \mathbf{C}$ NMR $\left(75.47 \mathbf{~ M H z}, \mathbf{C D C l}_{3}\right): \delta(\mathrm{ppm}) 160.0\left(\mathrm{~d},{ }^{1} J_{\mathrm{C}-\mathrm{F}}=270 \mathrm{~Hz}, 1 \mathrm{C}, \mathrm{C}_{6}\right), 158.1$ (s, 1C, $\left.\mathrm{C}_{13}\right), 133.1\left(\mathrm{~d},{ }^{3} J_{\mathrm{C}-\mathrm{F}}=2 \mathrm{~Hz}, 1 \mathrm{C}, \mathrm{C}_{4}\right), 131.0\left(\mathrm{~s}, 1 \mathrm{C}, \mathrm{C}_{10}\right), 129.7\left(\mathrm{~s}, 2 \mathrm{C}, \mathrm{C}_{11}\right), 128.6\left(\mathrm{~m}, 4 \mathrm{C}, \mathrm{C}_{2,3}\right)$, $127.3\left(\mathrm{~d},{ }^{6} J_{\mathrm{C}-\mathrm{F}}=2 \mathrm{~Hz}, 1 \mathrm{C}, \mathrm{C}_{1}\right), 113.9\left(\mathrm{~s}, 2 \mathrm{C}, \mathrm{C}_{12}\right), 107.2\left(\mathrm{~d},{ }^{2} J_{\mathrm{C}-\mathrm{F}}=8 \mathrm{~Hz}, 1 \mathrm{C}, \mathrm{C}_{5}\right), 55.3\left(\mathrm{~s}, 1 \mathrm{C}, \mathrm{C}_{14}\right), 54.6$ $\left(\mathrm{d},{ }^{2} J_{\mathrm{C}-\mathrm{F}}=27 \mathrm{~Hz}, 1 \mathrm{C}, \mathrm{C}_{7}\right), 50.3\left(\mathrm{~s}, 1 \mathrm{C}, \mathrm{C}_{9}\right), 19.0\left(\mathrm{~s}, 1 \mathrm{C}, \mathrm{C}_{8}\right)$. IR (neat) $v 2933,2855,1687,1611,1585$, 1510, 1493, 1463, 1448, 1366, 1300, 1244, 1171, 1109, 1033, 915, 826, 752, $693 \mathrm{~cm}^{-1}$. HR-MS (ESI+): $\mathrm{m} / \mathrm{z}=286.1616[\mathrm{M}+\mathrm{H}]^{+}$, calcd. for $\mathrm{C}_{18} \mathrm{H}_{21} \mathrm{FNO}: 286.1607 .[\alpha]_{\mathrm{D}}^{25}=-40\left(c 0.5, \mathrm{CH}_{3} \mathrm{Cl}\right)$. Chiral HPLC: Method conditions: Col. Daicel Chiralpak ASH 4.6mmx250mm $5 \mu \mathrm{m}, 1 \mathrm{~mL} / \mathrm{min}, 20^{\circ} \mathrm{C}, 95 \%$ n-heptane $05 \%$ propanol; peak $1: \operatorname{tr}=23.151 \mathrm{~min}, \%$ Area $=100 \% . R f=0.19\left(15 \% \mathrm{Et}_{2} \mathrm{O}\right.$ in pentane $)$.

(R,Z)-3-fluoro-N-(4-methoxybenzyl)-4-phenylbut-3-en-2-amine (7c):

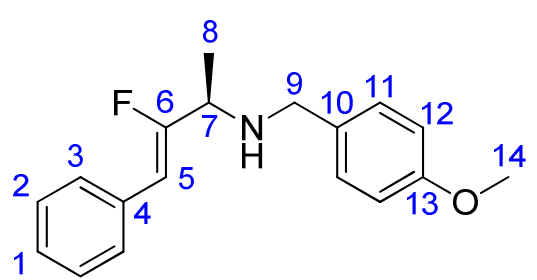

The general two steps procedure for the synthesis of secondary PMB amines using sulfinamide 4c (1 eq., $180 \mathrm{mg}, 668 \mu \mathrm{mol})$ afforded the (R,Z)-3-fluoro-N-(4-methoxybenzyl)-4-phenylbut-3en-2-amine (96 mg, $336 \mu \mathrm{mol}$, yield $51 \%$ ) as a colorless oil.

${ }^{1} \mathbf{H}$ NMR (400.13 MHz, $\left.\mathbf{C D C l}_{3}\right): \delta(\mathrm{ppm}) 7.52\left(\mathrm{~d},{ }^{3} \mathrm{~J}_{\mathrm{H}-\mathrm{H}}=9 \mathrm{~Hz}, 2 \mathrm{H}, \mathrm{H}_{3}\right), 7.37-7.22\left(\mathrm{~m}, 5 \mathrm{H}, \mathrm{H}_{1,2,11}\right), 6.87$ $\left(\mathrm{d},{ }^{3} J_{\mathrm{H}-\mathrm{H}}=9 \mathrm{~Hz}, 2 \mathrm{H}, \mathrm{H}_{12}\right), 5.65\left(\mathrm{~d},{ }^{3} J_{\mathrm{H}-\mathrm{F}}=39 \mathrm{~Hz}, 1 \mathrm{H}, \mathrm{H}_{5}\right), 3.80\left(\mathrm{~s}, 3 \mathrm{H}, \mathrm{H}_{14}\right), 3.84\left(\mathrm{~d},{ }^{3} J_{\mathrm{H}-\mathrm{H}}=13 \mathrm{~Hz}, 1 \mathrm{H}\right.$, $\left.\mathrm{H}_{9}\right), 3.68\left(\mathrm{~d},{ }^{3} J_{\mathrm{H}-\mathrm{H}}=13 \mathrm{~Hz}, 1 \mathrm{H}, \mathrm{H}_{9}\right), 3.38\left(\mathrm{dquad},{ }^{3} J_{\mathrm{H}-\mathrm{H}}=7 \mathrm{~Hz},{ }^{3} J_{\mathrm{H}-\mathrm{F}}=22 \mathrm{~Hz}, 1 \mathrm{H}, \mathrm{H}_{7}\right), 1.75$ (br. s, $1 \mathrm{H}$, $\mathrm{NH}), 1.36\left(\mathrm{~d},{ }^{3} J_{\mathrm{H}-\mathrm{H}}=7 \mathrm{~Hz}, 3 \mathrm{H}, \mathrm{H}_{8}\right) .{ }^{19} \mathbf{F}$ NMR $\left(\mathbf{3 7 6 . 5 0} \mathbf{~ M H z}, \mathbf{C D C l}_{3}\right): \delta(\mathrm{ppm})-118.43\left(\mathrm{dd},{ }^{3} J_{\mathrm{F}-\mathrm{H}}=22 \mathrm{~Hz}\right.$, $\left.{ }^{3} J_{\mathrm{F}-\mathrm{H}}=39 \mathrm{~Hz}, 1 \mathrm{~F}, \mathrm{~F}_{6}\right) .{ }^{13} \mathbf{C}$ NMR $\left(\mathbf{7 5 . 4 7} \mathbf{~ M H z}, \mathbf{C D C l}_{3}\right): \delta(\mathrm{ppm}) 160.8\left(\mathrm{~d},{ }^{1} J_{\mathrm{C}-\mathrm{F}}=270 \mathrm{~Hz}, 1 \mathrm{C}, \mathrm{C}_{6}\right), 158.8$ (s, 1C, $\mathrm{C}_{13}$ ), 133.3 (d, $\left.{ }^{3} J_{\mathrm{C}-\mathrm{F}}=2 \mathrm{~Hz}, 1 \mathrm{C}, \mathrm{C}_{4}\right), 132.0$ (s, 1C, $\mathrm{C}_{10}$ ), 129.4 (s, 2C, $\mathrm{C}_{11}$ ), $128.6\left(\mathrm{~m}, 4 \mathrm{C}, \mathrm{C}_{2,3}\right.$ ), $127.1\left(\mathrm{~d},{ }^{6} J_{\mathrm{C}-\mathrm{F}}=2 \mathrm{~Hz}, 1 \mathrm{C}, \mathrm{C}_{1}\right), 113.9\left(\mathrm{~s}, 2 \mathrm{C}, \mathrm{C}_{12}\right), 106.6\left(\mathrm{~d},{ }^{2} J_{\mathrm{C}-\mathrm{F}}=8 \mathrm{~Hz}, 1 \mathrm{C}, \mathrm{C}_{5}\right), 55.3\left(\mathrm{~s}, 1 \mathrm{C}, \mathrm{C}_{14}\right), 54.7$ $\left(\mathrm{d},{ }^{2} J_{\mathrm{C}-\mathrm{F}}=27 \mathrm{~Hz}, 1 \mathrm{C}, \mathrm{C}_{7}\right), 50.5$ (s, 1C, C ), 19.4 (s, 1C, $\mathrm{C}_{8}$ ). IR (neat) $v 2933,2835,1687,1611,1586$, 1511, 1494, 1464, 1448, 1367, 1300, 1245, 1172, 1111, 1034, 916, 828, 736, $694 \mathrm{~cm}^{-1}$. HR-MS (ESI+): 
$\mathrm{m} / \mathrm{z}=286.1617[\mathrm{M}+\mathrm{H}]^{+}$, calcd. for $\mathrm{C}_{18} \mathrm{H}_{21} \mathrm{FNO}: 286.1607 .[\alpha]_{\mathrm{D}}^{25}=+44\left(c 0.8, \mathrm{CH}_{3} \mathrm{Cl}\right)$. Chiral HPLC: Method conditions: Col. Daicel Chiralpak ASH 4.6mmx250mm $5 \mu \mathrm{m}, 1 \mathrm{~mL} / \mathrm{min}, 2{ }^{\circ} \mathrm{C}, 95 \% \mathrm{n}$-heptane $05 \%$ propanol; peak 1: $\operatorname{tr}=30.735 \mathrm{~min}, \%$ Area $=100 \% . R f=0.19\left(15 \% \mathrm{Et}_{2} \mathrm{O}\right.$ in pentane $)$.

Mixture of diastereoisomers: Chiral HPLC: Method conditions: Col. Daicel Chiralpak ASH $4.6 \mathrm{mmx} 250 \mathrm{~mm} 5 \mu \mathrm{m}, 1 \mathrm{~mL} / \mathrm{min}, 20^{\circ} \mathrm{C}, 95 \%$ n-heptane $05 \%$ propanol; peak $1: \operatorname{tr}=22.458 \mathrm{~min}, \%$ Area $=43.63 \%$; peak $2: \operatorname{tr}=29.672 \mathrm{~min}, \%$ Area $=56.37 \%$.

(S,Z)-2-fluoro-N-(4-methoxybenzyl)-1,3-diphenylprop-2-en-1-amine (7d):<smiles>[3H]Oc1ccc(CNC(/C(F)=C/c2ccccc2)(c2ccccc2)c2ccccc2)cc1</smiles>

The general two steps procedure for the synthesis of secondary PMB amines using sulfinamide 4d (1 eq., $626 \mathrm{mg}, 1.9 \mathrm{mmol}$ ) afforded the (S,Z)-2-fluoro- $N$-(4-methoxybenzyl)-1,3diphenylprop-2-en-1-amine (460 $\mathrm{mg}, 1.3 \mathrm{mmol}$, yield $70 \%$ ) as a colorless oil.

${ }^{1} \mathbf{H}$ NMR (400.13 MHz, $\left.\mathbf{C D C l}_{3}\right): \delta(\mathrm{ppm}) 7.53\left(\mathrm{~d},{ }^{3} J_{\mathrm{H}-\mathrm{H}}=9 \mathrm{~Hz}, 2 \mathrm{H}, \mathrm{H}_{3}\right), 7.48\left(\mathrm{~d},{ }^{3} J_{\mathrm{H}-\mathrm{H}}=8 \mathrm{~Hz}, 2 \mathrm{H}, \mathrm{H}_{9}\right)$,

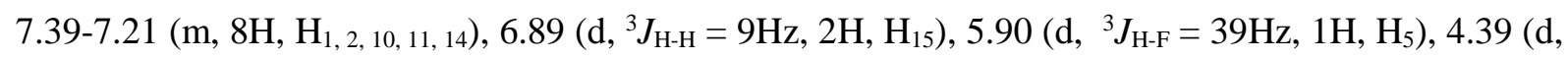
$\left.{ }^{3} J_{\mathrm{H}-\mathrm{F}}=17 \mathrm{~Hz}, 1 \mathrm{H}, \mathrm{H}_{7}\right), 3.85\left(\mathrm{~d},{ }^{3} J_{\mathrm{H}-\mathrm{F}}=13 \mathrm{~Hz}, 1 \mathrm{H}, \mathrm{H}_{12}\right), 3.81\left(\mathrm{~S}, 3 \mathrm{H}, \mathrm{H}_{17}\right), 3.78\left(\mathrm{~d},{ }^{3} J_{\mathrm{H}-\mathrm{F}}=13 \mathrm{~Hz}, 1 \mathrm{H}, \mathrm{H}_{12}\right)$, 1.82 (br.s, $1 \mathrm{H}, \mathrm{NH}) .{ }^{19} \mathbf{F}$ NMR (376.50 MHz, $\left.\mathbf{C D C l}_{3}\right): \delta(\mathrm{ppm})-112.59\left(\mathrm{dd},{ }^{3} J_{\mathrm{F}-\mathrm{H}}=17 \mathrm{~Hz},{ }^{3} J_{\mathrm{F}-\mathrm{H}}=39 \mathrm{~Hz}\right.$, 1F, $\left.\mathrm{F}_{6}\right) .{ }^{13} \mathbf{C}$ NMR (75.47 MHz, $\left.\mathbf{C D C l}_{3}\right): \delta(\mathrm{ppm}) 159.5\left(\mathrm{~d},{ }^{1} J_{\mathrm{C}-\mathrm{F}}=269 \mathrm{~Hz}, 1 \mathrm{C}, \mathrm{C}_{6}\right), 158.8\left(\mathrm{C}_{16}\right), 139.7$

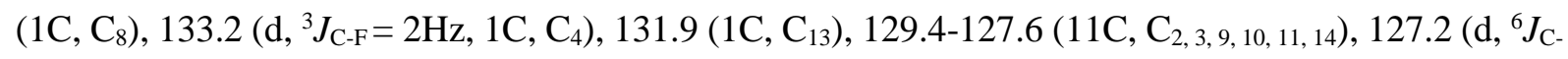
$\left.\mathrm{F}=2 \mathrm{~Hz}, 1 \mathrm{C}, \mathrm{C}_{1}\right), 113.9\left(2 \mathrm{C}, \mathrm{C}_{15}\right), 107.3\left(\mathrm{~d},{ }^{2} J_{\mathrm{C}-\mathrm{F}}=7 \mathrm{~Hz}, 1 \mathrm{C}, \mathrm{C}_{5}\right), 63.3\left(\mathrm{~d},{ }^{2} J_{\mathrm{C}-\mathrm{F}}=29 \mathrm{~Hz}, 1 \mathrm{C}, \mathrm{C}_{7}\right), 55.3(1 \mathrm{C}$, $\mathrm{C}_{17}$ ), $50.8\left(\mathrm{~s}, 1 \mathrm{C}, \mathrm{C}_{12}\right.$ ). IR (neat) $v 3027,2932,2835,1687,1611,1585,1511,1494,1448,1301,1244$, 1173, 1100, 1031, 915, 893, 826, 753, $693 \mathrm{~cm}^{-1}$. HR-MS (ESI+): m/z = $348.1761[\mathrm{M}+\mathrm{H}]^{+}$, calcd. for $\mathrm{C}_{23} \mathrm{H}_{23} \mathrm{FNO}$ : $348.1764 .[\alpha]_{\mathrm{D}}^{25}=+51\left(c\right.$ 0.35, $\left.\mathrm{CH}_{3} \mathrm{Cl}\right)$. Chiral HPLC: Method conditions: Col. Daicel Chiralpak ASH $4.6 \mathrm{mmx} 250 \mathrm{~mm} 5 \mu \mathrm{m}, 1 \mathrm{~mL} / \mathrm{min}, 20^{\circ} \mathrm{C}, 95 \%$ n-heptane $05 \%$ propanol; peak $1: \operatorname{tr}=6.181$ $\min , \%$ Area $=100 \% . R f=0.28\left(15 \% \mathrm{Et}_{2} \mathrm{O}\right.$ in pentane $)$.

(Z)-2-fluoro-N-(4-methoxybenzyl)-1,3-diphenylprop-2-en-1-amine (7d+e): 


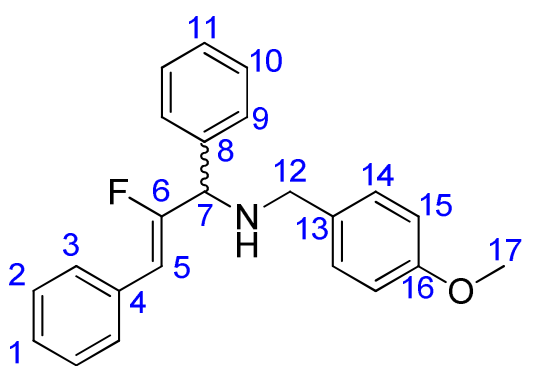

The general two steps procedure for the synthesis of secondary PMB amines using sulfinamides $\mathbf{4 d}+\mathbf{e}(1$ eq., $850 \mathrm{mg}, 2.6 \mathrm{mmol})$ afforded the (Z)-2-fluoro- $N$-(4-methoxybenzyl)-1,3diphenylprop-2-en-1-amine (614 mg, $1.8 \mathrm{mmol}$, yield $69 \%)$ as a colorless oil.

${ }^{1} \mathbf{H}$ NMR (400.13 MHz, $\left.\mathbf{C D C l}_{3}\right): \delta(\mathrm{ppm}) 7.55\left(\mathrm{~d},{ }^{3} J_{\mathrm{H}-\mathrm{H}}=9 \mathrm{~Hz}, 2 \mathrm{H}, \mathrm{H}_{3}\right), 7.50\left(\mathrm{~d},{ }^{3} J_{\mathrm{H}-\mathrm{H}}=8 \mathrm{~Hz}, 2 \mathrm{H}, \mathrm{H}_{9}\right)$,

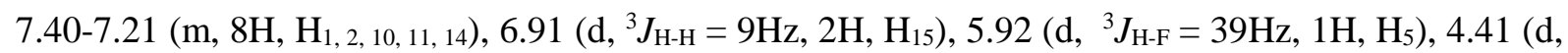
$\left.{ }^{3} J_{\mathrm{H}-\mathrm{F}}=17 \mathrm{~Hz}, 1 \mathrm{H}, \mathrm{H}_{7}\right), 3.86\left(\mathrm{~d},{ }^{3} J_{\mathrm{H}-\mathrm{F}}=13 \mathrm{~Hz}, 1 \mathrm{H}, \mathrm{H}_{12}\right), 3.81\left(\mathrm{~S}, 3 \mathrm{H}, \mathrm{H}_{17}\right), 3.79\left(\mathrm{~d},{ }^{3} J_{\mathrm{H}-\mathrm{F}}=13 \mathrm{~Hz}, 1 \mathrm{H}, \mathrm{H}_{12}\right)$, 1.92 (br.s, $1 \mathrm{H}, \mathrm{NH}) .{ }^{19} \mathbf{F}$ NMR (376.50 MHz, $\left.\mathbf{C D C l}_{3}\right): \delta(\mathrm{ppm})-112.42\left(\mathrm{dd},{ }^{3} J_{\mathrm{F}-\mathrm{H}}=17 \mathrm{~Hz},{ }^{3} J_{\mathrm{F}-\mathrm{H}}=39 \mathrm{~Hz}\right.$, 1F, $\left.\mathrm{F}_{6}\right) .{ }^{13} \mathbf{C}$ NMR (75.47 MHz, $\left.\mathbf{C D C l}_{3}\right): \delta(\mathrm{ppm}) 159.4\left(\mathrm{~d},{ }^{1} J_{\mathrm{C}-\mathrm{F}}=269 \mathrm{~Hz}, 1 \mathrm{C}, \mathrm{C}_{6}\right), 158.8\left(\mathrm{C}_{16}\right), 139.7$ $\left(1 \mathrm{C}, \mathrm{C}_{8}\right), 133.2\left(\mathrm{~d},{ }^{3} J_{\mathrm{C}-\mathrm{F}}=2 \mathrm{~Hz}, 1 \mathrm{C}, \mathrm{C}_{4}\right), 131.9\left(1 \mathrm{C}, \mathrm{C}_{13}\right), 129.4-127.6\left(11 \mathrm{C}, \mathrm{C}_{2,3,9,10,11,14)}\right) 127.2\left(\mathrm{~d},{ }^{6} J_{\mathrm{C}-}\right.$ $\left.\mathrm{F}=2 \mathrm{~Hz}, 1 \mathrm{C}, \mathrm{C}_{1}\right), 113.9\left(2 \mathrm{C}, \mathrm{C}_{15}\right), 107.3\left(\mathrm{~d},{ }^{2} J_{\mathrm{C}-\mathrm{F}}=7 \mathrm{~Hz}, 1 \mathrm{C}, \mathrm{C}_{5}\right), 63.3\left(\mathrm{~d},{ }^{2} J_{\mathrm{C}-\mathrm{F}}=29 \mathrm{~Hz}, 1 \mathrm{C}, \mathrm{C}_{7}\right), 55.3(1 \mathrm{C}$, $\left.\mathrm{C}_{17}\right), 50.8\left(\mathrm{~s}, 1 \mathrm{C}, \mathrm{C}_{12}\right.$ ). IR (neat) $v 3027,2933,2835,1687,1611,1585,1511,1494,1449,1300,1245$, 1173, 1101, 1032, 916, 826, 753, $694 \mathrm{~cm}^{-1}$. HR-MS (ESI+): m/z = $348.1765[\mathrm{M}+\mathrm{H}]^{+}$, calcd. for $\mathrm{C}_{23} \mathrm{H}_{23} \mathrm{FNO}$ : 348.1764. Chiral HPLC: Method conditions: Col. Daicel Chiralpak ASH 4.6mmx250mm $5 \mu \mathrm{m}, 1 \mathrm{~mL} / \mathrm{min}, 20^{\circ} \mathrm{C}, 95 \%$ n-heptane $05 \%$ propanol; peak $1: \operatorname{tr}=6.071 \mathrm{~min}, \%$ Area $=50.04 \%$; peak 2 : $\operatorname{tr}=7.042 \mathrm{~min}, \%$ Area $=49.96 \% . R f=0.28\left(15 \% \mathrm{Et}_{2} \mathrm{O}\right.$ in pentane $)$.

(S,Z)-2-fluoro-N-(4-methoxybenzyl)-4-methyl-1-phenylpent-1-en-3-amine (7f):

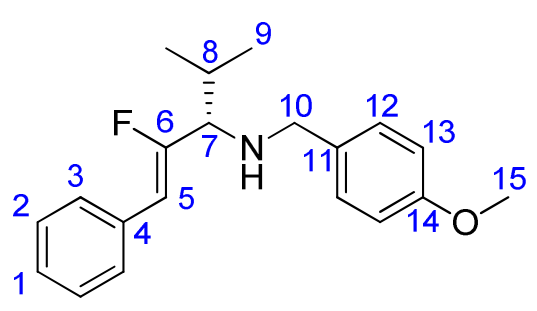

The general two steps procedure for the synthesis of secondary PMB amines using sulfinamide $4 \mathbf{f}$ (1 eq., $210 \mathrm{mg}, 706 \mu \mathrm{mol})$ afforded the (S,Z)-2-fluoro-N-(4-methoxybenzyl)-4-methyl-1phenylpent-1-en-3-amine (148 mg, $472 \mu \mathrm{mol}$, yield $66 \%)$ as a colorless oil.

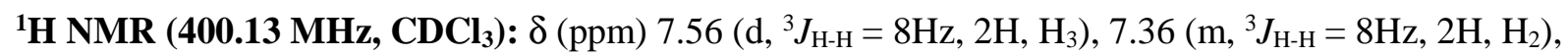
$7.30\left(\mathrm{~d},{ }^{3} J_{\mathrm{H}-\mathrm{H}}=9 \mathrm{~Hz}, 2 \mathrm{H}, \mathrm{H}_{12}\right), 7.25\left(\mathrm{~m}, 1 \mathrm{H}, \mathrm{H}_{1}\right), 6.89\left(\mathrm{~d},{ }^{3} J_{\mathrm{H}-\mathrm{H}}=9 \mathrm{~Hz}, 2 \mathrm{H}, \mathrm{H}_{13}\right), 5.59\left(\mathrm{~d},{ }^{3} J_{\mathrm{H}-\mathrm{F}}=39 \mathrm{~Hz}\right.$, $\left.1 \mathrm{H}, \mathrm{H}_{5}\right), 3.90\left(\mathrm{~d},{ }^{3} J_{\mathrm{H}-\mathrm{H}}=13 \mathrm{~Hz}, 1 \mathrm{H}, \mathrm{H}_{10}\right), 3.81\left(\mathrm{~s}, 3 \mathrm{H}, \mathrm{H}_{15}\right), 3.67\left(\mathrm{~d},{ }^{3} J_{\mathrm{H}-\mathrm{H}}=13 \mathrm{~Hz}, 1 \mathrm{H}, \mathrm{H}_{10}\right), 2.83\left(\mathrm{dd},{ }^{3} J_{\mathrm{H}-}\right.$ $\left.\mathrm{H}=7 \mathrm{~Hz},{ }^{3} J_{\mathrm{H}-\mathrm{F}}=25 \mathrm{~Hz}, 1 \mathrm{H}, \mathrm{H}_{7}\right), 1.91\left(\mathrm{~m}, 1 \mathrm{H}, \mathrm{H}_{8}\right), 1.55$ (br. s, $\left.1 \mathrm{H}, \mathrm{NH}\right), 1.08\left(\mathrm{~d},{ }^{3} J_{\mathrm{H}-\mathrm{H}}=7 \mathrm{~Hz}, 3 \mathrm{H}, \mathrm{H}_{9}\right)$, $0.99\left(\mathrm{~d},{ }^{3} J_{\mathrm{H}-\mathrm{H}}=7 \mathrm{~Hz}, 3 \mathrm{H}, \mathrm{H}_{9}\right) .{ }^{19} \mathbf{F} \mathbf{N M R}\left(376.50 \mathbf{~ M H z}, \mathbf{C D C l}_{3}\right): \delta(\mathrm{ppm})-116.08\left(\mathrm{dd},{ }^{3} J_{\mathrm{F}-\mathrm{H}}=25 \mathrm{~Hz},{ }^{3} J_{\mathrm{F}-}\right.$ $\left.\mathrm{H}=39 \mathrm{~Hz}, 1 \mathrm{~F}, \mathrm{~F}_{6}\right) .{ }^{13} \mathbf{C}$ NMR $\left(\mathbf{7 5 . 4 7} \mathbf{~ M H z}, \mathbf{C D C l}_{3}\right): \delta(\mathrm{ppm}) 159.3\left(\mathrm{~d},{ }^{1} J_{\mathrm{C}-\mathrm{F}}=271 \mathrm{~Hz}, 1 \mathrm{C}, \mathrm{C}_{6}\right), 158.6(\mathrm{~s}$, $\left.1 \mathrm{C}, \mathrm{C}_{14}\right), 133.4\left(\mathrm{~d},{ }^{3} J_{\mathrm{C}-\mathrm{F}}=2 \mathrm{~Hz}, 1 \mathrm{C}, \mathrm{C}_{4}\right), 132.5\left(\mathrm{~s}, 1 \mathrm{C}, \mathrm{C}_{11}\right), 129.4-128-5\left(\mathrm{~s}, 6 \mathrm{C}, \mathrm{C}_{2,3,12}\right), 127.0\left(\mathrm{~d},{ }^{6} J_{\mathrm{C}-\mathrm{F}}=\right.$ $\left.2 \mathrm{~Hz}, 1 \mathrm{C}, \mathrm{C}_{1}\right), 113.8\left(\mathrm{~s}, 2 \mathrm{C}, \mathrm{C}_{13}\right), 108.4\left(\mathrm{~d},{ }^{2} J_{\mathrm{C}-\mathrm{F}}=7 \mathrm{~Hz}, 1 \mathrm{C}, \mathrm{C}_{5}\right), 65.7\left(\mathrm{~d},{ }^{2} J_{\mathrm{C}-\mathrm{F}}=25 \mathrm{~Hz}, 1 \mathrm{C}, \mathrm{C}_{7}\right), 55.2(\mathrm{~s}$, $1 \mathrm{C}, \mathrm{C}_{15}$ ), 50.6 (s, 1C, $\mathrm{C}_{10}$ ), 31.1 (s, 1C, $\mathrm{C}_{8}$ ), 19.8 (s, 1C, C 9 ), 19.7 (s, 1C, C 9 ). IR (neat) v 2958, 2835, 1683, 1611, 1586, 1511, 1463, 1448, 1385, 1365, 1300, 1243, 1171, 1100, 1034, 960, 915, 824, 752, 
$693 \mathrm{~cm}^{-1}$. HR-MS (ESI+): $\mathrm{m} / \mathrm{z}=314.1933[\mathrm{M}+\mathrm{H}]^{+}$, calcd. for $\mathrm{C}_{20} \mathrm{H}_{25} \mathrm{FNO}: 314.1920$. $[\alpha]_{\mathrm{D}}^{25}=+102(c$ $\left.1.05, \mathrm{CH}_{3} \mathrm{Cl}\right) . R f=0.17\left(15 \% \mathrm{Et}_{2} \mathrm{O}\right.$ in pentane $)$.

(R,Z)-2-fluoro-N-(4-methoxybenzyl)-4-methyl-1-phenylpent-1-en-3-amine (7g):<smiles>COc1ccc(CNC(C=Cc2ccccc2)C(C)C)cc1</smiles>

The general two steps procedure for the synthesis of secondary PMB amines using sulfinamide $\mathbf{4 g}(1$ eq., $140 \mathrm{mg}, 471 \mu \mathrm{mol})$ afforded the (R,Z)-2-fluoro-N-(4-methoxybenzyl)-4-methyl-1phenylpent-1-en-3-amine ( $85 \mathrm{mg}, 272 \mu \mathrm{mol}$, yield $58 \%$ ) as a colorless oil.

${ }^{1} \mathbf{H}$ NMR (400.13 MHz, CDCl 3 ): $\delta(\mathrm{ppm}) 7.55\left(\mathrm{~d},{ }^{3} J_{\mathrm{H}-\mathrm{H}}=8 \mathrm{~Hz}, 2 \mathrm{H}, \mathrm{H}_{3}\right), 7.37\left(\mathrm{t},{ }^{3} J_{\mathrm{H}-\mathrm{H}}=8 \mathrm{~Hz}, 2 \mathrm{H}, \mathrm{H}_{2}\right)$, $7.29\left(\mathrm{~d},{ }^{3} J_{\mathrm{H}-\mathrm{H}}=9 \mathrm{~Hz}, 2 \mathrm{H}, \mathrm{H}_{12}\right), 7.25\left(\mathrm{~m}, 1 \mathrm{H}, \mathrm{H}_{1}\right), 6.88\left(\mathrm{~d},{ }^{3} J_{\mathrm{H}-\mathrm{H}}=9 \mathrm{~Hz}, 2 \mathrm{H}, \mathrm{H}_{13}\right), 5.59\left(\mathrm{~d},{ }^{3} J_{\mathrm{H}-\mathrm{F}}=39 \mathrm{~Hz}\right.$, $\left.1 \mathrm{H}, \mathrm{H}_{5}\right), 3.90\left(\mathrm{~d},{ }^{3} J_{\mathrm{H}-\mathrm{H}}=13 \mathrm{~Hz}, 1 \mathrm{H}, \mathrm{H}_{10}\right), 3.81\left(\mathrm{~s}, 3 \mathrm{H}, \mathrm{H}_{15}\right), 3.66\left(\mathrm{~d},{ }^{3} J_{\mathrm{H}-\mathrm{H}}=13 \mathrm{~Hz}, 1 \mathrm{H}, \mathrm{H}_{10}\right), 2.83\left(\mathrm{dd},{ }^{3} J_{\mathrm{H}-}\right.$ $\left.\mathrm{H}=7 \mathrm{~Hz},{ }^{3} J_{\mathrm{H}-\mathrm{F}}=25 \mathrm{~Hz}, 1 \mathrm{H}, \mathrm{H}_{7}\right), 1.91\left(\mathrm{~m}, 1 \mathrm{H}, \mathrm{H}_{8}\right), 1.72($ br. s, $1 \mathrm{H}, \mathrm{NH}), 1.07\left(\mathrm{~d},{ }^{3} J_{\mathrm{H}-\mathrm{H}}=7 \mathrm{~Hz}, 3 \mathrm{H}, \mathrm{H}_{9}\right)$, $0.98\left(\mathrm{~d},{ }^{3} J_{\mathrm{H}-\mathrm{H}}=7 \mathrm{~Hz}, 3 \mathrm{H}, \mathrm{H}_{9}\right) .{ }^{19} \mathbf{F}$ NMR (376.50 MHz, $\left.\mathbf{C D C l}_{3}\right): \delta(\mathrm{ppm})-116.08\left(\mathrm{dd},{ }^{3} \boldsymbol{J}_{\mathrm{F}-\mathrm{H}}=25 \mathrm{~Hz},{ }^{3} J_{\mathrm{F}-}\right.$ $\left.\mathrm{H}=39 \mathrm{~Hz}, 1 \mathrm{~F}, \mathrm{~F}_{6}\right) .{ }^{13} \mathbf{C} \mathbf{N M R}\left(\mathbf{7 5 . 4 7} \mathbf{~ M H z}, \mathbf{C D C l}_{3}\right): \delta(\mathrm{ppm}) 159.3\left(\mathrm{~d},{ }^{1} J_{\mathrm{C}-\mathrm{F}}=271 \mathrm{~Hz}, 1 \mathrm{C}, \mathrm{C}_{6}\right), 158.6(\mathrm{~s}$, $\left.1 \mathrm{C}, \mathrm{C}_{14}\right), 133.4\left(\mathrm{~d},{ }^{3} J_{\mathrm{C}-\mathrm{F}}=2 \mathrm{~Hz}, 1 \mathrm{C}, \mathrm{C}_{4}\right), 132.4\left(\mathrm{~s}, 1 \mathrm{C}, \mathrm{C}_{11}\right), 129.4-128-5\left(\mathrm{~s}, 6 \mathrm{C}, \mathrm{C}_{2,3,12}\right), 127.0\left(\mathrm{~d},{ }^{6} J_{\mathrm{C}-\mathrm{F}}=\right.$ $\left.2 \mathrm{~Hz}, 1 \mathrm{C}, \mathrm{C}_{1}\right), 113.7\left(\mathrm{~s}, 2 \mathrm{C}, \mathrm{C}_{13}\right), 108.5\left(\mathrm{~d},{ }^{2} J_{\mathrm{C}-\mathrm{F}}=7 \mathrm{~Hz}, 1 \mathrm{C}, \mathrm{C}_{5}\right), 65.7\left(\mathrm{~d},{ }^{2} J_{\mathrm{C}-\mathrm{F}}=25 \mathrm{~Hz}, 1 \mathrm{C}, \mathrm{C}_{7}\right), 55.3(\mathrm{~s}$, $1 \mathrm{C}, \mathrm{C}_{15}$ ), $50.6\left(\mathrm{~s}, 1 \mathrm{C}, \mathrm{C}_{10}\right.$ ), $31.1\left(\mathrm{~s}, 1 \mathrm{C}, \mathrm{C}_{8}\right), 19.8$ (s, 1C, $\mathrm{C}_{9}$ ), 19.7 (s, 1C, $\mathrm{C}_{9}$ ). IR (neat) v 2957, 2835, $1683,1611,1585,1510,1463,1448,1385,1365,1300,1243,1171,1100,1034,915,825,753,693$, $663 \mathrm{~cm}^{-1}$. HR-MS (ESI+): $\mathrm{m} / \mathrm{z}=314.1935[\mathrm{M}+\mathrm{H}]^{+}$, calcd. for $\mathrm{C}_{20} \mathrm{H}_{25} \mathrm{FNO}: 314.1920 .[\alpha]_{\mathrm{D}}^{25}=-116.8$ (c $\left.0.95, \mathrm{CH}_{3} \mathrm{Cl}\right) . R f=0.17\left(15 \% \mathrm{Et}_{2} \mathrm{O}\right.$ in pentane).

(Z)-3-fluoro-N-(4-methoxybenzyl)-1,4-diphenylbut-3-en-2-amine (7h):

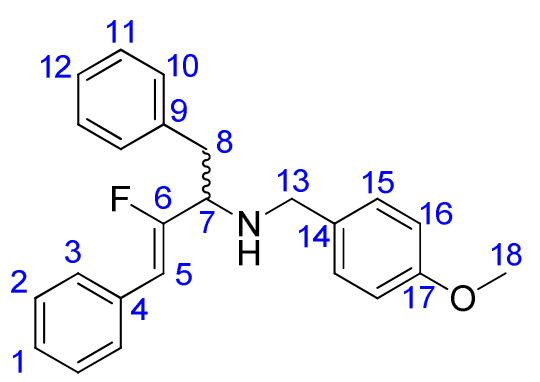

The general two steps procedure for the synthesis of secondary PMB amines using sulfinamide $4 \mathrm{~h}$ (1 eq., $244 \mathrm{mg}, 706 \mu \mathrm{mol})$ afforded the (Z)-3-fluoro-N-(4-methoxybenzyl)-1,4-diphenylbut3-en-2-amine (250 mg, $692 \mu \mathrm{mol}$, yield $98 \%$ ) as a colorless oil. 
${ }^{1} \mathbf{H}$ NMR (400.13 MHz, CDCl $): \delta(\mathrm{ppm}) 7.51\left(\mathrm{~d},{ }^{3} J_{\mathrm{H}-\mathrm{H}}=8 \mathrm{~Hz}, 2 \mathrm{H}, \mathrm{H}_{3}\right), 7.36\left(\mathrm{t},{ }^{3} J_{\mathrm{H}-\mathrm{H}}=8 \mathrm{~Hz}, 2 \mathrm{H}, \mathrm{H}_{2}\right)$, $7.26\left(\mathrm{~m}, 8 \mathrm{H}, \mathrm{H}_{1,10,11,12}\right), 7.13\left(\mathrm{~d},{ }^{3} J_{\mathrm{H}-\mathrm{H}}=9 \mathrm{~Hz}, 2 \mathrm{H}, \mathrm{H}_{15}\right), 6.84\left(\mathrm{~d},{ }^{3} J_{\mathrm{H}-\mathrm{H}}=9 \mathrm{~Hz}, 2 \mathrm{H}, \mathrm{H}_{16}\right), 5.57\left(\mathrm{~d},{ }^{3} J_{\mathrm{H}-\mathrm{F}}=\right.$ $\left.40 \mathrm{~Hz}, 1 \mathrm{H}, \mathrm{H}_{5}\right), 3.88\left(\mathrm{~d},{ }^{3} J_{\mathrm{H}-\mathrm{H}}=13 \mathrm{~Hz}, 1 \mathrm{H}, \mathrm{H}_{13}\right), 3.81\left(\mathrm{~s}, 3 \mathrm{H}, \mathrm{H}_{18}\right), 3.66\left(\mathrm{~d},{ }^{3} J_{\mathrm{H}-\mathrm{H}}=13 \mathrm{~Hz}, 1 \mathrm{H}, \mathrm{H}_{13}\right), 3.42$ $\left(\mathrm{dt},{ }^{3} J_{\mathrm{H}-\mathrm{H}}=7 \mathrm{~Hz},{ }^{3} J_{\mathrm{H}-\mathrm{F}}=22 \mathrm{~Hz}, 1 \mathrm{H}, \mathrm{H}_{7}\right), 3.07\left(\mathrm{dd},{ }^{3} J_{\mathrm{H}-\mathrm{H}}=7 \mathrm{~Hz},{ }^{2} J_{\mathrm{H}-\mathrm{H}}=13 \mathrm{~Hz}, 1 \mathrm{H}, \mathrm{H}_{8}\right), 2.93\left(\mathrm{dd},{ }^{3} J_{\mathrm{H}-\mathrm{H}}=\right.$ $\left.7 \mathrm{~Hz},{ }^{2} J_{\mathrm{H}-\mathrm{H}}=13 \mathrm{~Hz}, 1 \mathrm{H}, \mathrm{H}_{8}\right), 1.63$ (br. s, $\left.1 \mathrm{H}, \mathbf{N H}\right) .{ }^{19} \mathbf{F}$ NMR (376.50 MHz, $\left.\mathbf{C D C l}_{3}\right): \delta(\mathrm{ppm})-117.85$ $\left(\mathrm{dd},{ }^{3} J_{\mathrm{F}-\mathrm{H}}=22 \mathrm{~Hz},{ }^{3} J_{\mathrm{F}-\mathrm{H}}=40 \mathrm{~Hz}, 1 \mathrm{~F}, \mathrm{~F}_{6}\right) .{ }^{13} \mathbf{C} \mathbf{N M R}\left(\mathbf{7 5 . 4 7} \mathbf{M H z}, \mathbf{C D C l}_{3}\right): \delta(\mathrm{ppm}) 159.0\left(\mathrm{~d},{ }^{1} J_{\mathrm{C}-\mathrm{F}}=\right.$ $\left.270 \mathrm{~Hz}, 1 \mathrm{C}, \mathrm{C}_{6}\right), 158.7$ (s, 1C, $\mathrm{C}_{17}$ ), 138.0 (s, 1C, $\mathrm{C}_{9}$ ), 133.2 (d, $\left.{ }^{3} J_{\mathrm{C}-\mathrm{F}}=2 \mathrm{~Hz}, 1 \mathrm{C}, \mathrm{C}_{4}\right), 132.0$ (s, 1C, $\mathrm{C}_{14}$ ), 129.3-128.5 (s, 10C, $\mathrm{C}_{2,3,10,11,15)}, 126.6\left(\mathrm{~d},{ }^{6} J_{\mathrm{C}-\mathrm{F}}=2 \mathrm{~Hz}, 1 \mathrm{C}, \mathrm{C}_{1}\right), 126.6\left(\mathrm{~s}, 1 \mathrm{C}, \mathrm{C}_{12}\right), 113.8\left(\mathrm{~s}, 2 \mathrm{C}, \mathrm{C}_{16}\right)$, $108.0\left(\mathrm{~d},{ }^{2} J_{\mathrm{C}-\mathrm{F}}=7 \mathrm{~Hz}, 1 \mathrm{C}, \mathrm{C}_{5}\right), 61.0\left(\mathrm{~d},{ }^{2} J_{\mathrm{C}-\mathrm{F}}=27 \mathrm{~Hz}, 1 \mathrm{C}, \mathrm{C}_{7}\right), 55.3\left(\mathrm{~s}, 1 \mathrm{C}, \mathrm{C}_{18}\right), 50.5\left(\mathrm{~s}, 1 \mathrm{C}, \mathrm{C}_{13}\right), 39.8(\mathrm{~s}$, $1 \mathrm{C}, \mathrm{C}_{8}$ ). IR (neat) v 3060, 3027, 2932, 2835, 1713, 1687, 1611, 1585, 1510, 1495, 1453, 1362, 1300, 1244, 1173, 1104, 1032, 915, 827, 736, $693 \mathrm{~cm}^{-1}$. HR-MS (ESI+): m/z = $362.1904[\mathrm{M}+\mathrm{H}]^{+}$, calcd. for $\mathrm{C}_{24} \mathrm{H}_{25} \mathrm{FNO}$ : 362.1920. $R f=0.28\left(15 \% \mathrm{Et}_{2} \mathrm{O}\right.$ in pentane).

\section{e. Synthesis of the PMB-protected bisolefins 3}

\section{General procedure:}

In an oven dried round-bottom flask equipped with a stir bar and flushed with argon, a solution of the starting secondary amine (1 eq.), triethylamine (2 eq.) and a solution (50\% in EtOAc) of T3P ${ }^{\circledR}(1.5$ eq.) in DCM was prepared. Carboxylic acid (1.2 eq.) was finally added to the mixture. The mixture was stirred at room temperature and the reaction was controlled by TLC and ${ }^{19} \mathrm{~F}$ NMR. When full consumption of starting material was observed, the reaction was stopped by adding water and the aqueous layer extracted with DCM. The organic layers were combined and dried over $\mathrm{MgSO}_{4}$. After filtration and evaporation of the volatile materials under reduced pressure, the crude was purified by flash chromatography on silica gel (Pentane: $\mathrm{Et}_{2} \mathrm{O}$ ) to afford the desired PMB protected bisolefin.

(S,Z)-N-(3-fluoro-4-phenylbut-3-en-2-yl)-N-(4-methoxybenzyl)but-3-enamide (3b):

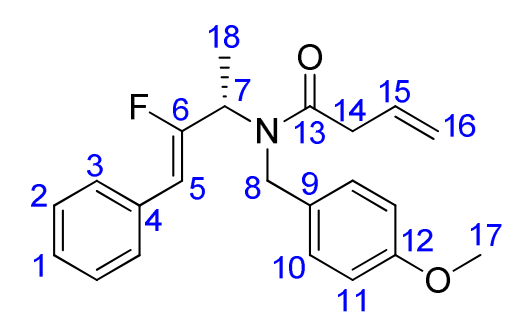

yield $82 \%$ ) as a light yellow oil.

The general procedure for the synthesis of the PMB protected bisolefins using secondary amine $7 \mathbf{b}$ (1 eq., $53 \mathrm{mg}, 186 \mu \mathrm{mol})$ followed by a purification by chromatography over silica gel (eluent: pentane: $\left.\mathrm{Et}_{2} \mathrm{O}=50: 50\right)$ afforded the $(\boldsymbol{S}, \boldsymbol{Z})$ - N-(3-fluoro-4-phenylbut3-en-2-yl)-N-(4-methoxybenzyl)but-3-enamide (65 mg, $151 \mu \mathrm{mol}$, . 
${ }^{1} \mathbf{H}$ NMR (400.13 MHz, CDCl 3 ): $\delta(\mathrm{ppm}) 7.30\left(\mathrm{~m}, 7 \mathrm{H}, \mathrm{H}_{1,2,3,10}\right.$ both rotamers), $6.87\left(\mathrm{~d},{ }^{3} J_{\mathrm{H}-\mathrm{H}}=9 \mathrm{~Hz}\right.$, $2 \mathrm{H}, \mathrm{H}_{11}$ rotamer 2), $6.79\left(\mathrm{~d},{ }^{3} \mathrm{~J}_{\mathrm{H}-\mathrm{H}}=9 \mathrm{~Hz}, 2 \mathrm{H}, \mathrm{H}_{11}\right.$ rotamer 1), $6.08\left(\mathrm{~m}, 1 \mathrm{H}, \mathrm{H}_{15}\right.$ rotamer 1), $5.99(\mathrm{~m}, 1 \mathrm{H}$, $\mathrm{H}_{15}$ rotamer 2), $5.75\left(\mathrm{~m}, 1 \mathrm{H}, \mathrm{H}_{7}\right.$ rotamer 2), $5.71\left(\mathrm{~d},{ }^{3} J_{\mathrm{H}-\mathrm{F}}=39 \mathrm{~Hz}, 1 \mathrm{H}, \mathrm{H}_{5}\right.$ rotamer 2$), 5.67\left(\mathrm{~d},{ }^{3} J_{\mathrm{H}-\mathrm{F}}=\right.$ $38 \mathrm{~Hz}, 1 \mathrm{H}, \mathrm{H}_{5}$ rotamer 1), $5.23\left(\mathrm{~m}, 2 \mathrm{H}, \mathrm{H}_{16}\right.$ rotamer 1), $5.11\left(\mathrm{~m}, 2 \mathrm{H}, \mathrm{H}_{16}\right.$ rotamer 2), $4.80\left(\mathrm{~m}, 1 \mathrm{H}, \mathrm{H}_{7}\right.$ rotamer 1), 4.54 (m, 2H, $\mathrm{H}_{8}$ both rotamers), 3.80 (s, $3 \mathrm{H}, \mathrm{H}_{17}$ rotamer 2$), 3.76$ (s, $3 \mathrm{H}, \mathrm{H}_{17}$ rotamer 1), 3.37 $\left(\mathrm{d},{ }^{3} J_{\mathrm{H}-\mathrm{H}}=6 \mathrm{~Hz}, 2 \mathrm{H}, \mathrm{H}_{14}\right.$ rotamer 1$), 3.11\left(\mathrm{~m}, \mathrm{H}_{14}\right.$ rotamer 2$), 1.37\left(\mathrm{~d},{ }^{3} J_{\mathrm{H}-\mathrm{H}}=7 \mathrm{~Hz}, 3 \mathrm{H}, \mathrm{H}_{14}\right.$ rotamer 1$)$, $1.32\left(\mathrm{~d},{ }^{3} J_{\mathrm{H}-\mathrm{H}}=7 \mathrm{~Hz}, 3 \mathrm{H}, \mathrm{H}_{18}\right.$ rotamer 2). ${ }^{19} \mathbf{F} \mathbf{N M R}\left(\mathbf{3 7 6 . 5 0} \mathbf{M H z}, \mathbf{C D C l}_{\mathbf{3}}\right): \delta(\mathrm{ppm})-106.71\left(\mathrm{dd},{ }^{3} \boldsymbol{J}_{\mathrm{F}-\mathrm{H}}=\right.$ $5 \mathrm{~Hz},{ }^{3} J_{\mathrm{F}-\mathrm{H}}=39 \mathrm{~Hz}, 1 \mathrm{~F}, \mathrm{~F}_{6}$ rotamer 1$),-107.90\left(\mathrm{dd},{ }^{3} J_{\mathrm{F}-\mathrm{H}}=12 \mathrm{~Hz},{ }^{3} J_{\mathrm{F}-\mathrm{H}}=39 \mathrm{~Hz}, 1 \mathrm{~F}, \mathrm{~F}_{6}\right.$ rotamer 2$) .{ }^{13} \mathrm{C}$ NMR (75.47 MHz, $\left.\mathbf{C D C l}_{3}\right)$ : $\delta(\mathrm{ppm}) 172.2\left(\mathrm{C}_{13}\right.$ rotamer 2$), 171.7\left(\mathrm{C}_{13}\right.$ rotamer 1$), 158.8\left(\mathrm{C}_{12}\right.$ rotamer 2), $158.6\left(\mathrm{~d},{ }^{1} J_{\mathrm{C}-\mathrm{F}}=269 \mathrm{~Hz}, \mathrm{C}_{6}\right.$ rotamer 2$), 158.5\left(\mathrm{C}_{12}\right.$ rotamer 1$), 157.6\left(\mathrm{~d},{ }^{1} J_{\mathrm{C}-\mathrm{F}}=269 \mathrm{~Hz}, \mathrm{C}_{6}\right.$ rotamer 1$)$, $132.7\left(\mathrm{~d},{ }^{3} J_{\mathrm{C}-\mathrm{F}}=2 \mathrm{~Hz}, \mathrm{C}_{4}\right.$ rotamer 2$), 132.3\left(\mathrm{~d},{ }^{3} J_{\mathrm{C}-\mathrm{F}}=3 \mathrm{~Hz}, \mathrm{C}_{4}\right.$ rotamer 1$), 131.6\left(\mathrm{C}_{15}\right.$ rotamer 2$), 131.1$ $\left(\mathrm{C}_{15}\right.$ rotamer 1$), 129.8$ - $127.1\left(\mathrm{C}_{1,2,3,9,10}\right.$ both rotamers $), 118.1$ ( $\mathrm{C}_{16}$ rotamer 1$), 117.8\left(\mathrm{C}_{16}\right.$ rotamer 2$)$, $114.2\left(\mathrm{C}_{11}\right.$ rotamer 2), $113.7\left(\mathrm{C}_{11}\right.$ rotamer 1$), 108.5\left(\mathrm{~d},{ }^{2} J_{\mathrm{C}-\mathrm{F}}=8 \mathrm{~Hz}, \mathrm{C}_{5}\right.$ rotamer 2$), 108.2\left(\mathrm{~d},{ }^{2} J_{\mathrm{C}-\mathrm{F}}=8 \mathrm{~Hz}\right.$, $\mathrm{C}_{5}$ rotamer 1), $55.3\left(\mathrm{C}_{17}\right.$ rotamer 2$), 55.2\left(\mathrm{C}_{17}\right.$ rotamer 1$), 54.2\left(\mathrm{~d},{ }^{2} J_{\mathrm{C}-\mathrm{F}}=28 \mathrm{~Hz}, \mathrm{C}_{7}\right.$ rotamer 1$), 50.5(\mathrm{~d}$, ${ }^{2} J_{\mathrm{C}-\mathrm{F}}=26 \mathrm{~Hz}, \mathrm{C}_{7}$ rotamer 2$), 46.8\left(\mathrm{C}_{8}\right.$ rotamer 2$), 44.9\left(\mathrm{C}_{8}\right.$ rotamer 1$), 39.2\left(\mathrm{C}_{14}\right.$ both rotamers $), 17.0(\mathrm{~d}$, ${ }^{3} J_{\mathrm{C}-\mathrm{F}}=6 \mathrm{~Hz}, \mathrm{C}_{18}$ rotamer 1$), 15.6\left(\mathrm{~d},{ }^{3} J_{\mathrm{C}-\mathrm{F}}=6 \mathrm{~Hz}, \mathrm{C}_{18}\right.$ rotamer 2$) . \mathbf{I R}$ (neat) $v 2935,2836,1685,1646$, 1612, 1586, 1511, 1495, 1447, 1405, 1360, 1332, 1290, 1244, 1174, 1108, 1032, 994, 915, 856, 820, 753, $693 \mathrm{~cm}^{-1}$. HR-MS (ESI+): $\mathrm{m} / \mathrm{z}=354.1874[\mathrm{M}+\mathrm{H}]^{+}$, calcd. for $\mathrm{C}_{22} \mathrm{H}_{25} \mathrm{FNO}_{2}: 354.1869$. [ $\left.\boldsymbol{\alpha}\right]_{\mathbf{D}}^{25}=-$ $68\left(c 0.5, \mathrm{CH}_{3} \mathrm{Cl}\right)$. Chiral HPLC: Method conditions: Col. Daicel Chiralpak ASH 4.6mmx250mm 5um, $1 \mathrm{~mL} / \mathrm{min}, 20^{\circ} \mathrm{C}, 80 \%$ n-heptane $20 \%$ propanol; peak $1: \operatorname{tr}=6.924 \mathrm{~min}, \%$ Area $=0.82 \%$; peak $2: \operatorname{tr}=$ $7.678 \mathrm{~min}, \%$ Area $=99.15 \% . R f=0.24\left(40 \% \mathrm{Et}_{2} \mathrm{O}\right.$ in pentane $)$.

(R,Z)-N-(3-fluoro-4-phenylbut-3-en-2-yl)-N-(4-methoxybenzyl)but-3-enamide (3c):

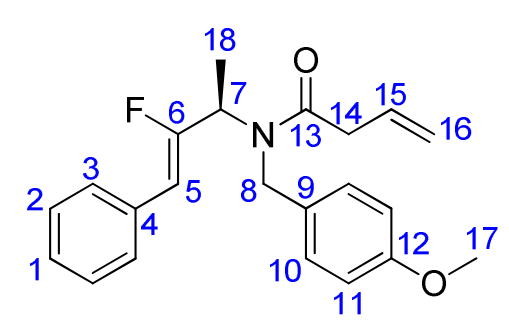

yield $74 \%$ ) as a light yellow oil.
The general procedure for the synthesis of the PMB protected bisolefins using secondary amine $7 \mathrm{c}$ ( 1 eq., $35 \mathrm{mg}, 123 \mu \mathrm{mol})$ followed by a purification by chromatography over silica gel (eluent: pentane: $\left.\mathrm{Et}_{2} \mathrm{O}=50: 50\right)$ afforded the $(\boldsymbol{R}, \boldsymbol{Z})-\mathrm{N}$-(3-fluoro-4-phenylbut3-en-2-yl)-N-(4-methoxybenzyl)but-3-enamide (39 mg, $91 \mu \mathrm{mol}$, 
${ }^{1} \mathbf{H}$ NMR (400.13 MHz, CDCl 3 ): $\delta(\mathrm{ppm}) 7.30\left(\mathrm{~m}, 7 \mathrm{H}, \mathrm{H}_{1,2,3,10}\right.$ both rotamers), $6.87\left(\mathrm{~d},{ }^{3} J_{\mathrm{H}-\mathrm{H}}=9 \mathrm{~Hz}\right.$, $2 \mathrm{H}, \mathrm{H}_{11}$ rotamer 2), $6.79\left(\mathrm{~d},{ }^{3} \mathrm{~J}_{\mathrm{H}-\mathrm{H}}=9 \mathrm{~Hz}, 2 \mathrm{H}, \mathrm{H}_{11}\right.$ rotamer 1), $6.05\left(\mathrm{~m}, 1 \mathrm{H}, \mathrm{H}_{15}\right.$ rotamer 1), $5.98(\mathrm{~m}, 1 \mathrm{H}$, $\mathrm{H}_{15}$ rotamer 2), $5.75\left(\mathrm{~m}, 1 \mathrm{H}, \mathrm{H}_{7}\right.$ rotamer 2), $5.70\left(\mathrm{~d},{ }^{3} J_{\mathrm{H}-\mathrm{F}}=39 \mathrm{~Hz}, 1 \mathrm{H}, \mathrm{H}_{5}\right.$ rotamer 2$), 5.67\left(\mathrm{~d},{ }^{3} J_{\mathrm{H}-\mathrm{F}}=\right.$ $38 \mathrm{~Hz}, 1 \mathrm{H}, \mathrm{H}_{5}$ rotamer 1), $5.22\left(\mathrm{~m}, 2 \mathrm{H}, \mathrm{H}_{16}\right.$ rotamer 1), $5.10\left(\mathrm{~m}, 2 \mathrm{H}, \mathrm{H}_{16}\right.$ rotamer 2), $4.80\left(\mathrm{~m}, 1 \mathrm{H}, \mathrm{H}_{7}\right.$ rotamer 1), 4.54 (m, 2H, $\mathrm{H}_{8}$ both rotamers), 3.79 (s, $3 \mathrm{H}, \mathrm{H}_{17}$ rotamer 2$), 3.75$ (s, $3 \mathrm{H}, \mathrm{H}_{17}$ rotamer 1), 3.35 $\left(\mathrm{d},{ }^{3} J_{\mathrm{H}-\mathrm{H}}=6 \mathrm{~Hz}, 2 \mathrm{H}, \mathrm{H}_{14}\right.$ rotamer 1$), 3.10\left(\mathrm{~m}, \mathrm{H}_{14}\right.$ rotamer 2$), 1.35\left(\mathrm{~d},{ }^{3} J_{\mathrm{H}-\mathrm{H}}=7 \mathrm{~Hz}, 3 \mathrm{H}, \mathrm{H}_{14}\right.$ rotamer 1$)$, $1.31\left(\mathrm{~d},{ }^{3} J_{\mathrm{H}-\mathrm{H}}=7 \mathrm{~Hz}, 3 \mathrm{H}, \mathrm{H}_{18}\right.$ rotamer 2). ${ }^{19} \mathbf{F} \mathbf{N M R}\left(\mathbf{3 7 6 . 5 0} \mathbf{M H z}, \mathbf{C D C l}_{\mathbf{3}}\right): \delta(\mathrm{ppm})-106.71\left(\mathrm{dd},{ }^{3} \boldsymbol{J}_{\mathrm{F}-\mathrm{H}}=\right.$ $5 \mathrm{~Hz},{ }^{3} J_{\mathrm{F}-\mathrm{H}}=40 \mathrm{~Hz}, 1 \mathrm{~F}, \mathrm{~F}_{6}$ rotamer 1$),-107.90\left(\mathrm{dd},{ }^{3} J_{\mathrm{F}-\mathrm{H}}=12 \mathrm{~Hz},{ }^{3} J_{\mathrm{F}-\mathrm{H}}=40 \mathrm{~Hz}, 1 \mathrm{~F}, \mathrm{~F}_{6}\right.$ rotamer 2$) .{ }^{13} \mathrm{C}$ NMR (75.47 MHz, $\left.\mathbf{C D C l}_{3}\right)$ : $\delta(\mathrm{ppm}) 172.2\left(\mathrm{C}_{13}\right.$ rotamer 2$), 171.7\left(\mathrm{C}_{13}\right.$ rotamer 1$), 158.8\left(\mathrm{C}_{12}\right.$ rotamer 2), $158.6\left(\mathrm{~d},{ }^{1} J_{\mathrm{C}-\mathrm{F}}=269 \mathrm{~Hz}, \mathrm{C}_{6}\right.$ rotamer 2$), 158.5\left(\mathrm{C}_{12}\right.$ rotamer 1$), 157.6\left(\mathrm{~d},{ }^{1} J_{\mathrm{C}-\mathrm{F}}=269 \mathrm{~Hz}, \mathrm{C}_{6}\right.$ rotamer 1$)$, $132.7\left(\mathrm{~d},{ }^{3} J_{\mathrm{C}-\mathrm{F}}=2 \mathrm{~Hz}, \mathrm{C}_{4}\right.$ rotamer 2$), 132.3\left(\mathrm{~d},{ }^{3} J_{\mathrm{C}-\mathrm{F}}=3 \mathrm{~Hz}, \mathrm{C}_{4}\right.$ rotamer 1$), 131.6\left(\mathrm{C}_{15}\right.$ rotamer 2$), 131.1$ $\left(\mathrm{C}_{15}\right.$ rotamer 1$), 129.8$ - $127.1\left(\mathrm{C}_{1,2,3,9,10}\right.$ both rotamers $), 118.1$ ( $\mathrm{C}_{16}$ rotamer 1$), 117.8\left(\mathrm{C}_{16}\right.$ rotamer 2$)$, $114.2\left(\mathrm{C}_{11}\right.$ rotamer 2), $113.7\left(\mathrm{C}_{11}\right.$ rotamer 1$), 108.5\left(\mathrm{~d},{ }^{2} J_{\mathrm{C}-\mathrm{F}}=8 \mathrm{~Hz}, \mathrm{C}_{5}\right.$ rotamer 2$), 108.2\left(\mathrm{~d},{ }^{2} J_{\mathrm{C}-\mathrm{F}}=8 \mathrm{~Hz}\right.$, $\mathrm{C}_{5}$ rotamer 1), $55.3\left(\mathrm{C}_{17}\right.$ rotamer 2$), 55.2\left(\mathrm{C}_{17}\right.$ rotamer 1$), 54.2\left(\mathrm{~d},{ }^{2} J_{\mathrm{C}-\mathrm{F}}=28 \mathrm{~Hz}, \mathrm{C}_{7}\right.$ rotamer 1$), 50.5(\mathrm{~d}$, ${ }^{2} J_{\mathrm{C}-\mathrm{F}}=26 \mathrm{~Hz}, \mathrm{C}_{7}$ rotamer 2$), 46.8\left(\mathrm{C}_{8}\right.$ rotamer 2$), 44.9\left(\mathrm{C}_{8}\right.$ rotamer 1$), 39.2\left(\mathrm{C}_{14}\right.$ both rotamers $), 17.0(\mathrm{~d}$, ${ }^{3} J_{\mathrm{C}-\mathrm{F}}=6 \mathrm{~Hz}, \mathrm{C}_{18}$ rotamer 1$), 15.6\left(\mathrm{~d},{ }^{3} J_{\mathrm{C}-\mathrm{F}}=6 \mathrm{~Hz}, \mathrm{C}_{18}\right.$ rotamer 2$) . \mathbf{I R}$ (neat) $v 2934,2836,1685,1646$, 1612, 1586, 1511, 1495, 1447, 1405, 1360, 1332, 1290, 1244, 1174, 1108, 1032, 994, 915, 856, 821, 753, 735, $693 \mathrm{~cm}^{-1}$. HR-MS (ESI+): $\mathrm{m} / \mathrm{z}=354.1882[\mathrm{M}+\mathrm{H}]^{+}$, calcd. for $\mathrm{C}_{22} \mathrm{H}_{25} \mathrm{FNO}_{2}: 354.1869$. $[\boldsymbol{\alpha}]_{\mathbf{D}}^{25}=+58\left(c 0.5, \mathrm{CH}_{3} \mathrm{Cl}\right)$. Chiral HPLC: Method conditions: Col. Daicel Chiralpak ASH $4.6 \mathrm{~mm} \times 250 \mathrm{~mm} 5 \mu \mathrm{m}, 1 \mathrm{~mL} / \mathrm{min}, 20^{\circ} \mathrm{C}, 80 \%$ n-heptane $20 \%$ propanol; peak $1: \operatorname{tr}=6.910 \mathrm{~min}, \%$ Area $=$ $100 \% . R f=0.24\left(40 \% \mathrm{Et}_{2} \mathrm{O}\right.$ in pentane).

Mixture of diastereoisomers: Chiral HPLC: Method conditions: Col. Daicel Chiralpak ASH $4.6 \mathrm{~mm} \times 250 \mathrm{~mm} 5 \mu \mathrm{m}, 1 \mathrm{~mL} / \mathrm{min}, 20^{\circ} \mathrm{C}, 80 \%$ n-heptane $20 \%$ propanol; peak $1: \operatorname{tr}=6.908 \mathrm{~min}, \%$ Area $=$ $47.92 \%$; peak $2: \operatorname{tr}=7.669 \mathrm{~min}, \%$ Area $=52.08 \%$.

\section{(S,Z)-N-(2-fluoro-1,3-diphenylallyl)- $N$-(4-methoxybenzyl)but-3-enamide (3d):}

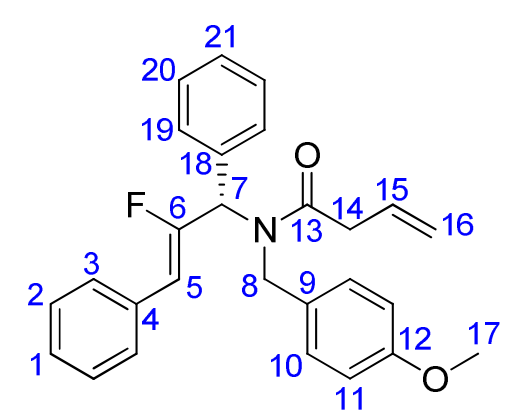

The general procedure for the synthesis of the PMB protected bisolefins using secondary amine 7d (1 eq., $300 \mathrm{mg}, 864 \mu \mathrm{mol})$ followed by a purification by chromatography over silica gel (eluent: pentane: $\left.\mathrm{Et}_{2} \mathrm{O}=60: 40\right)$ afforded the (S,Z)- $N$-(2-fluoro-1,3diphenylallyl)- $N$-(4-methoxybenzyl)but-3-enamide (360 mg, 838 $\mu$ mol, yield $97 \%$ ) as a colorless oil. 
${ }^{1} \mathbf{H}$ NMR (400.13 MHz, $\left.\mathbf{C D C l}_{3}\right): \delta(\mathrm{ppm}) 7.32$ (m, 10H, $\mathrm{H}_{1,2,3,19,20,21}$ both rotamers), $7.02\left(\mathrm{~d},{ }^{3} \boldsymbol{J}_{\mathrm{H}-\mathrm{H}}=9\right.$ $\mathrm{Hz}, 2 \mathrm{H}, \mathrm{H}_{10}$ rotamer 2), $6.99\left(\mathrm{~d},{ }^{3} \mathrm{~J}_{\mathrm{H}-\mathrm{H}}=9 \mathrm{~Hz}, 2 \mathrm{H}, \mathrm{H}_{10}\right.$ rotamer 1$), 6.79\left(\mathrm{~m}, 1 \mathrm{H}, \mathrm{H}_{7}\right.$ rotamer 2$), 6.78(\mathrm{~d}$, ${ }^{3} J_{\mathrm{H}-\mathrm{H}}=8 \mathrm{~Hz}, 2 \mathrm{H}, \mathrm{H}_{11}$ rotamer 2), $6.67\left(\mathrm{~d},{ }^{3} J_{\mathrm{H}-\mathrm{H}}=8 \mathrm{~Hz}, 2 \mathrm{H}, \mathrm{H}_{11}\right.$ rotamer 1$), 6.01\left(\mathrm{~m}, 1 \mathrm{H}, \mathrm{H}_{15}\right.$ both rotamers), $5.90\left(\mathrm{~d},{ }^{3} J_{\mathrm{H}-\mathrm{F}}=39 \mathrm{~Hz}, 1 \mathrm{H}, \mathrm{H}_{5}\right.$ rotamer 2$), 5.82\left(\mathrm{~m}, 1 \mathrm{H}, \mathrm{H}_{7}\right.$ rotamer 1$), 5.67\left(\mathrm{~d},{ }^{3} J_{\mathrm{H}-\mathrm{F}}=39 \mathrm{~Hz}\right.$, $1 \mathrm{H}, \mathrm{H}_{5}$ rotamer 1), 5.13 (m, 2H, $\mathrm{H}_{16}$ both rotamers), 4.63 (m, $2 \mathrm{H}, \mathrm{H}_{8}$ both rotamers), $3.76\left(\mathrm{~s}, 3 \mathrm{H}, \mathrm{H}_{17}\right.$ rotamer 2), $3.74\left(\mathrm{~s}, 3 \mathrm{H}, \mathrm{H}_{17}\right.$ rotamer 1), $3.26\left(\mathrm{~d},{ }^{3} J_{\mathrm{H}-\mathrm{H}}=6 \mathrm{~Hz}, 2 \mathrm{H}, \mathrm{H}_{14}\right.$ rotamer 1), $3.13\left(\mathrm{~m}, \mathrm{H}_{14}\right.$ rotamer 2). ${ }^{19} \mathbf{F}$ NMR (376.50 $\left.\mathbf{~ M H z}, \mathbf{C D C l}_{3}\right): \delta(\mathrm{ppm})-104.57\left(\mathrm{dd},{ }^{3} J_{\mathrm{F}-\mathrm{H}}=1 \mathrm{~Hz},{ }^{3} J_{\mathrm{F}-\mathrm{H}}=38 \mathrm{~Hz}, 1 \mathrm{~F}, \mathrm{~F}_{6}\right.$ rotamer 1$)$, $-108.55\left(\mathrm{dd},{ }^{3} J_{\mathrm{F}-\mathrm{H}}=20 \mathrm{~Hz},{ }^{3} J_{\mathrm{F}-\mathrm{H}}=38 \mathrm{~Hz}, 1 \mathrm{~F}, \mathrm{~F}_{6}\right.$ rotamer 2$) .{ }^{13} \mathbf{C ~ N M R}\left(\mathbf{7 5 . 4 7} \mathbf{~ M H z}, \mathbf{C D C l}_{3}\right): \delta(\mathrm{ppm})$ $172.5\left(\mathrm{C}_{13}\right.$ rotamer 2$), 172.2\left(\mathrm{C}_{13}\right.$ rotamer 1$), 158.5\left(\mathrm{C}_{12}\right.$ both rotamers $), 156.5\left(\mathrm{~d},{ }^{1} J_{\mathrm{C}-\mathrm{F}}=270 \mathrm{~Hz}, \mathrm{C}_{6}\right.$ both rotamers), 135.9 ( $\mathrm{s}, \mathrm{C}_{18}$ rotamer 2$), 135.3$ ( $\mathrm{s}, \mathrm{C}_{18}$ rotamer 1$), 132.5$ (s, $\mathrm{C}_{4}$ rotamer 2$), 132.0$ ( $\mathrm{s}, \mathrm{C}_{4}$ rotamer 1), 131.4 ( $\mathrm{C}_{15}$ both rotamers), 129.3 - 126.8 ( $\mathrm{C}_{1,2,3,9,10,19,20,21}$ both rotamers), 118.1 ( $\mathrm{C}_{16}$ both rotamers), $113.9\left(\mathrm{C}_{11}\right.$ rotamer 2$), 113.3\left(\mathrm{C}_{11}\right.$ rotamer 1$), 111.3\left(\mathrm{~d},{ }^{2} J_{\mathrm{C}-\mathrm{F}}=6 \mathrm{~Hz}, \mathrm{C}_{5}\right.$ rotamer 2$), 111.1\left(\mathrm{~m}, \mathrm{C}_{5}\right.$ rotamer 1), $63.0\left(\mathrm{~d},{ }^{2} J_{\mathrm{C}-\mathrm{F}}=29 \mathrm{~Hz}, \mathrm{C}_{7}\right.$ rotamer 1$), 59.1\left(\mathrm{~d},{ }^{2} J_{\mathrm{C}-\mathrm{F}}=25 \mathrm{~Hz}, \mathrm{C}_{7}\right.$ rotamer 2$), 55.1\left(\mathrm{C}_{17}\right.$ both rotamers $)$, $48.6\left(\mathrm{C}_{8}\right.$ rotamer 2$), 47.2$ ( $\mathrm{C}_{8}$ rotamer 1$), 39.0$ ( $\mathrm{C}_{14}$ both rotamers). IR (neat) $v 3028,2934,2836,1650$, 1612, 1585, 1511, 1495, 1449, 1401, 1355, 1288, 1245, 1199, 1174, 1132, 1108, 1078, 1031, 992, 955, 915, 809, 752, 693, $660 \mathrm{~cm}^{-1}$. HR-MS (ESI+): $\mathrm{m} / \mathrm{z}=416.2014[\mathrm{M}+\mathrm{H}]^{+}$, calcd. for $\mathrm{C}_{27} \mathrm{H}_{27} \mathrm{FNO}_{2}$ : 416.2026. $[\boldsymbol{\alpha}]_{\mathbf{D}}^{25}=-92\left(c 1.0, \mathrm{CH}_{3} \mathrm{Cl}\right)$. Chiral HPLC: Method conditions: Col. Daicel Chiralpak ASH $4.6 \mathrm{~mm} \times 250 \mathrm{~mm} 5 \mu \mathrm{m}, 1 \mathrm{~mL} / \mathrm{min}, 20^{\circ} \mathrm{C}, 80 \%$ n-heptane $20 \%$ propanol; peak 1: $\operatorname{tr}=12.564 \mathrm{~min}, \%$ Area $=99.20 \%$; peak $2: \operatorname{tr}=14.250 \mathrm{~min}, \%$ Area $=0.8 \% . R f=0.31\left(50 \% \mathrm{Et}_{2} \mathrm{O}\right.$ in pentane $)$.

Racemic (Z)-N-(2-fluoro-1,3-diphenylallyl)-N-(4-methoxybenzyl)but-3-enamide (3d+e):

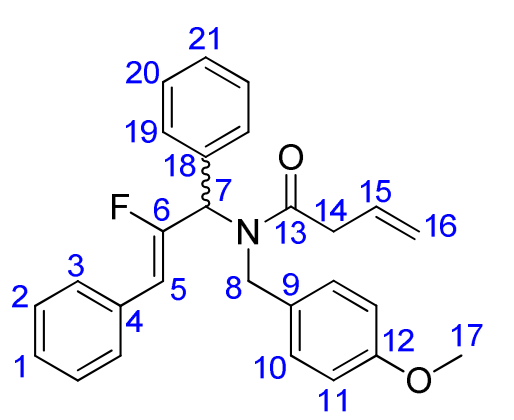

The general procedure for the synthesis of the PMB protected bisolefins using secondary amine $7 \mathbf{d}+\mathbf{e}(1$ eq., $100 \mathrm{mg}, 288 \mu \mathrm{mol})$ followed by a purification by chromatography over silica gel (eluent: pentane: $\left.\mathrm{Et}_{2} \mathrm{O}=60: 40\right)$ afforded the Racemic (Z)-N-(2-fluoro-1,3diphenylallyl)-N-(4-methoxybenzyl)but-3-enamide (115 mg, 268 $\mu$ mol, yield $93 \%$ ) as a colorless oil.

${ }^{1} \mathbf{H}$ NMR (400.13 MHz, $\left.\mathbf{C D C l}_{3}\right): \delta(\mathrm{ppm}) 7.32\left(\mathrm{~m}, 10 \mathrm{H}, \mathrm{H}_{1,2,3,19,20,21}\right.$ both rotamers), $7.02\left(\mathrm{~d},{ }^{3} J_{\mathrm{H}-\mathrm{H}}=9\right.$ $\mathrm{Hz}, 2 \mathrm{H}, \mathrm{H}_{10}$ both rotamers), $6.78\left(\mathrm{~d},{ }^{3} J_{\mathrm{H}-\mathrm{H}}=8 \mathrm{~Hz}, 2 \mathrm{H}, \mathrm{H}_{11}\right.$ rotamer 2$), 6.78\left(\mathrm{~m}, 1 \mathrm{H}, \mathrm{H}_{7}\right.$ rotamer 2$), 6.70$ 
(m, $2 \mathrm{H}, \mathrm{H}_{11}$ rotamer 1), $6.01\left(\mathrm{~m}, 1 \mathrm{H}, \mathrm{H}_{15}\right.$ both rotamers), $5.90\left(\mathrm{~d},{ }^{3} \mathrm{~J}_{\mathrm{H}-\mathrm{F}}=39 \mathrm{~Hz}, 1 \mathrm{H}, \mathrm{H}_{5}\right.$ rotamer 2), 5.82 (m, $1 \mathrm{H}, \mathrm{H}_{7}$ rotamer 1), $5.68\left(\mathrm{~d},{ }^{3} \mathrm{~J}_{\mathrm{H}-\mathrm{F}}=39 \mathrm{~Hz}, 1 \mathrm{H}, \mathrm{H}_{5}\right.$ rotamer 1), $5.13\left(\mathrm{~m}, 2 \mathrm{H}, \mathrm{H}_{16}\right.$ both rotamers $), 4.64$ (m, $2 \mathrm{H}, \mathrm{H}_{8}$ both rotamers), 3.76 (s, $3 \mathrm{H}, \mathrm{H}_{17}$ both rotamers), 3.19 (m, $\mathrm{H}_{14}$ both rotamers). ${ }^{19} \mathbf{F}$ NMR (376.50 MHz, $\left.\mathbf{C D C l}_{3}\right): \delta(\mathrm{ppm})-104.60\left(\mathrm{dd},{ }^{3} J_{\mathrm{F}-\mathrm{H}}=1 \mathrm{~Hz},{ }^{3} J_{\mathrm{F}-\mathrm{H}}=38 \mathrm{~Hz}, 1 \mathrm{~F}, \mathrm{~F}_{6}\right.$ rotamer 1), $-108.60(\mathrm{dd}$, ${ }^{3} J_{\mathrm{F}-\mathrm{H}}=20 \mathrm{~Hz},{ }^{3} J_{\mathrm{F}-\mathrm{H}}=39 \mathrm{~Hz}, 1 \mathrm{~F}, \mathrm{~F}_{6}$ rotamer 2). ${ }^{13} \mathbf{C ~ N M R}\left(\mathbf{7 5 . 4 7} \mathbf{~ M H z}, \mathbf{C D C l}_{3}\right): \delta(\mathrm{ppm}) 172.5\left(\mathrm{C}_{13}\right.$ both rotamers), 158.5 ( $\mathrm{C}_{12}$ both rotamers), $156.5\left(\mathrm{~d},{ }^{1} J_{\mathrm{C}-\mathrm{F}}=270 \mathrm{~Hz}, \mathrm{C}_{6}\right.$ both rotamers), 135.9 ( $\mathrm{s}, \mathrm{C}_{18}$ both

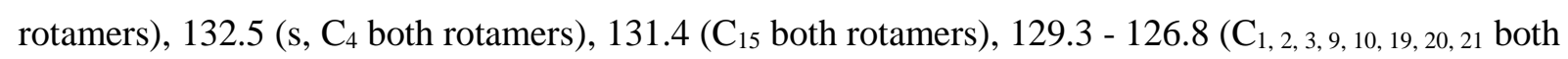
rotamers), 118.1 ( $\mathrm{C}_{16}$ both rotamers), $113.9\left(\mathrm{C}_{11}\right.$ rotamer 2$), 113.3\left(\mathrm{C}_{11}\right.$ rotamer 1$), 111.3\left(\mathrm{~d},{ }^{2} J_{\mathrm{C}-\mathrm{F}}=6 \mathrm{~Hz}\right.$, $\mathrm{C}_{5}$ both rotamers), $63.0\left(\mathrm{~m}, \mathrm{C}_{7}\right.$ rotamer 1), 59.1 ( $\mathrm{d},{ }^{2} J_{\mathrm{C}-\mathrm{F}}=25 \mathrm{~Hz}, \mathrm{C}_{7}$ rotamer 2$), 55.1$ ( $\mathrm{C}_{17}$ both rotamers), $48.6\left(\mathrm{C}_{8}\right.$ rotamer 2$), 47.2$ ( $\mathrm{C}_{8}$ rotamer 1$), 39.0$ ( $\mathrm{C}_{14}$ both rotamers). IR (neat) $v 3028,2934,2836,1650$, 1612, 1585, 1511, 1495, 1448, 1401, 1355, 1288, 1245, 1199, 1174, 1131, 1108, 1078, 1031, 993, 955, 916, 809, 752, $693 \mathrm{~cm}^{-1}$. HR-MS (ESI+): $\mathrm{m} / \mathrm{z}=416.2008[\mathrm{M}+\mathrm{H}]^{+}$, calcd. for $\mathrm{C}_{27} \mathrm{H}_{27} \mathrm{FNO}_{2}: 416.2026$.

Chiral HPLC: Method conditions: Col. Daicel Chiralpak ASH 4.6mmx $250 \mathrm{~mm} 5 \mu \mathrm{m}, 1 \mathrm{~mL} / \mathrm{min}, 20^{\circ} \mathrm{C}$, $80 \%$ n-heptane $20 \%$ propanol; peak $1: \operatorname{tr}=12.603 \mathrm{~min}, \%$ Area $=49.29 \%$; peak $2: \operatorname{tr}=14.261 \mathrm{~min}$, $\%$ Area $=50.71 \% . R f=0.31\left(50 \% \mathrm{Et}_{2} \mathrm{O}\right.$ in pentane $)$.

\section{(S,Z)-N-(2-fluoro-4-methyl-1-phenylpent-1-en-3-yl)-N-(4-methoxybenzyl)but-3-enamide (3f):}

The general procedure for the synthesis of the PMB protected bisolefins using secondary amine $7 \mathbf{f}$ (1<smiles>[3H]Oc1ccc(CN(C(=O)CC=C)C(C(F)=Cc2ccccc2)C(C)(C)C)cc1</smiles>
eq., $130 \mathrm{mg}, 415 \mu \mathrm{mol})$ followed by a purification by chromatography over silica gel (eluent: pentane: $\mathrm{Et}_{2} \mathrm{O}=60: 40$ ) afforded the (S,Z)-N-(2-fluoro-4-methyl-1-phenylpent-1-en-3-yl)-N(4-methoxybenzyl)but-3-enamide (95 mg, $221 \mu \mathrm{mol}$, yield $54 \%$ ) as a light yellow oil.

${ }^{1} \mathbf{H}$ NMR (400.13 MHz, $\left.\mathbf{C D C l}_{3}\right): \delta(\mathrm{ppm}) 7.25\left(\mathrm{~m}, 5 \mathrm{H}, \mathrm{H}_{1,2,3}\right.$ both rotamers), $7.12\left(\mathrm{~d},{ }^{3} J_{\mathrm{H}-\mathrm{H}}=9 \mathrm{~Hz}, 2 \mathrm{H}\right.$, $\mathrm{H}_{10}$ both rotamers), $6.78\left(\mathrm{~d},{ }^{3} \mathrm{~J}_{\mathrm{H}-\mathrm{H}}=9 \mathrm{~Hz}, 2 \mathrm{H}, \mathrm{H}_{11}\right.$ rotamer 2$), 6.72\left(\mathrm{~d},{ }^{3} \mathrm{~J}_{\mathrm{H}-\mathrm{H}}=9 \mathrm{~Hz}, 2 \mathrm{H}, \mathrm{H}_{11}\right.$ rotamer 1$)$, $6.08\left(\mathrm{~m}, 1 \mathrm{H}, \mathrm{H}_{15}\right.$ rotamer 1), $5.95\left(\mathrm{~m}, 1 \mathrm{H}, \mathrm{H}_{15}\right.$ rotamer 2$), 5.88\left(\mathrm{~d},{ }^{3} \mathrm{~J}_{\mathrm{H}-\mathrm{F}}=39 \mathrm{~Hz}, 1 \mathrm{H}, \mathrm{H}_{5}\right.$ rotamer 2$), 5.69$ $\left(\mathrm{d},{ }^{3} J_{\mathrm{H}-\mathrm{F}}=39 \mathrm{~Hz}, 1 \mathrm{H}, \mathrm{H}_{5}\right.$ rotamer 1), $5.23\left(\mathrm{~m}, 2 \mathrm{H}, \mathrm{H}_{16}\right.$ rotamer 1), $5.22\left(\mathrm{~m}, 1 \mathrm{H}, \mathrm{H}_{7}\right.$ rotamer 2$), 5.07(\mathrm{~m}$, $2 \mathrm{H}, \mathrm{H}_{16}$ rotamer 2), $4.61\left(\mathrm{~m}, 2 \mathrm{H}, \mathrm{H}_{8}\right.$ both rotamers), $4.09\left(\mathrm{dd},{ }^{3} J_{\mathrm{H}-\mathrm{H}}=11 \mathrm{~Hz},{ }^{3} J_{\mathrm{H}-\mathrm{F}}=20 \mathrm{~Hz}, 1 \mathrm{H}, \mathrm{H}_{7}\right.$ rotamer 1), 3.73 (s, $3 \mathrm{H}, \mathrm{H}_{17}$ rotamer 2), 3.71 (s, $3 \mathrm{H}, \mathrm{H}_{17}$ rotamer 1), 3.35 (m, $2 \mathrm{H}, \mathrm{H}_{14}$ rotamer 1), 3.05 (m, $\mathrm{H}_{14}$ rotamer 2), 2.22 (m, 1H, $\mathrm{H}_{18}$ both rotamers), 1.00 (m, $6 \mathrm{H}, \mathrm{H}_{19}$ both rotamers). ${ }^{\mathbf{1}} \mathbf{F}$ NMR (376.50 $\mathbf{~ M H z}$, $\left.\mathbf{C D C l}_{3}\right): \delta(\mathrm{ppm})-107.78\left(\mathrm{dd},{ }^{3} J_{\mathrm{F}-\mathrm{H}}=20 \mathrm{~Hz},{ }^{3} J_{\mathrm{F}-\mathrm{H}}=39 \mathrm{~Hz}, 1 \mathrm{~F}, \mathrm{~F}_{6}\right.$ rotamer 1$),-111.61\left(\mathrm{dd},{ }^{3} J_{\mathrm{F}-\mathrm{H}}=27 \mathrm{~Hz}\right.$, ${ }^{3} J_{\mathrm{F}-\mathrm{H}}=39 \mathrm{~Hz}, 1 \mathrm{~F}, \mathrm{~F}_{6}$ rotamer 2). ${ }^{\mathbf{1 3}} \mathbf{C}$ NMR $\left(\mathbf{7 5 . 4 7} \mathbf{~ M H z}, \mathbf{C D C l}_{3}\right): \delta(\mathrm{ppm}) 172.5\left(\mathrm{C}_{13}\right.$ rotamer 2), 171.6 $\left(\mathrm{C}_{13}\right.$ rotamer 1$), 158.6\left(\mathrm{C}_{12}\right.$ rotamer 2$), 158.3\left(\mathrm{C}_{12}\right.$ rotamer 1$), 157.4\left(\mathrm{~d},{ }^{1} J_{\mathrm{C}-\mathrm{F}}=272 \mathrm{~Hz}, \mathrm{C}_{6}\right.$ both rotamers $)$, $132.7\left(\mathrm{~d},{ }^{3} J_{\mathrm{C}-\mathrm{F}}=2 \mathrm{~Hz}, \mathrm{C}_{4}\right.$ rotamer 2$), 132.2\left(\mathrm{~d},{ }^{3} J_{\mathrm{C}-\mathrm{F}}=3 \mathrm{~Hz}, \mathrm{C}_{4}\right.$ rotamer 1$), 131.9\left(\mathrm{C}_{15}\right.$ rotamer 1$), 131.7$ $\left(\mathrm{C}_{15}\right.$ rotamer 2$), 129.8$ - $127.1\left(\mathrm{C}_{1,2,3,9,10}\right.$ both rotamers $), 118.1\left(\mathrm{C}_{16}\right.$ rotamer 1$), 117.9\left(\mathrm{C}_{16}\right.$ rotamer 2$)$, 
$114.1\left(\mathrm{C}_{11}\right.$ rotamer 2$), 113.5\left(\mathrm{C}_{11}\right.$ rotamer 1$), 110.7\left(\mathrm{~d},{ }^{2} J_{\mathrm{C}-\mathrm{F}}=7 \mathrm{~Hz}, \mathrm{C}_{5}\right.$ rotamer 2$), 110.4\left(\mathrm{~d},{ }^{2} J_{\mathrm{C}-\mathrm{F}}=8 \mathrm{~Hz}\right.$, $\mathrm{C}_{5}$ rotamer 1), $66.1\left(\mathrm{~d},{ }^{2} J_{\mathrm{C}-\mathrm{F}}=24 \mathrm{~Hz}, \mathrm{C}_{7}\right.$ rotamer 1$), 61.3\left(\mathrm{~d},{ }^{2} J_{\mathrm{C}-\mathrm{F}}=25 \mathrm{~Hz}, \mathrm{C}_{7}\right.$ rotamer 2$), 55.2\left(\mathrm{C}_{17}\right.$ rotamer 2), $55.1\left(\mathrm{C}_{17}\right.$ rotamer 1$), 47.1\left(\mathrm{~d},{ }^{4} J_{\mathrm{C}-\mathrm{F}}=3 \mathrm{~Hz}, \mathrm{C}_{8}\right.$ rotamer 2$), 45.7\left(\mathrm{~d},{ }^{4} J_{\mathrm{C}-\mathrm{F}}=3 \mathrm{~Hz}, \mathrm{C}_{8}\right.$ rotamer 1$), 39.4\left(\mathrm{C}_{14}\right.$ rotamer 1), $39.1\left(\mathrm{C}_{14}\right.$ rotamer 2), $27.7\left(\mathrm{~d},{ }^{3} J_{\mathrm{C}-\mathrm{F}}=3 \mathrm{~Hz}, \mathrm{C}_{18}\right.$ rotamer 1$), 26.9\left(\mathrm{~d},{ }^{3} J_{\mathrm{C}-\mathrm{F}}=2 \mathrm{~Hz}, \mathrm{C}_{18}\right.$ rotamer 2), 20.6 (3C, $\mathrm{C}_{19}$ rotamer 1), 20.0 (3C, $\mathrm{C}_{19}$ rotamer 2), 19.5 (3C, $\mathrm{C}_{19}$ rotamer 1), 19.1 (3C, $\mathrm{C}_{19}$ rotamer 2). IR (neat) $v 2964,1648,1613,1513,1465,1247,1176,1109,1034,968,916,810,753,695 \mathrm{~cm}^{-1}$. HR-MS (ESI+): $\mathrm{m} / \mathrm{z}=382.2189[\mathrm{M}+\mathrm{H}]^{+}$, calcd. for $\mathrm{C}_{24} \mathrm{H}_{29} \mathrm{FNO}_{2}: 382.2182$. $[\boldsymbol{\alpha}]_{\mathbf{D}}^{25}=-168$ (c 1.3, $\mathrm{CH}_{3} \mathrm{Cl}$ ). Chiral HPLC: Method conditions: Col. Daicel Chiralpak ASH 4.6mmx250mm $5 \mu \mathrm{m}$, $1 \mathrm{~mL} / \mathrm{min}, 20^{\circ} \mathrm{C}, 60 \%$ n-heptane $40 \%$ propanol; peak $1: \operatorname{tr}=3.773 \mathrm{~min}, \%$ Area $=100 \% . R f=0.27(40 \%$ $\mathrm{Et}_{2} \mathrm{O}$ in pentane).

\section{(R,Z)-N-(2-fluoro-4-methyl-1-phenylpent-1-en-3-yl)-N-(4-methoxybenzyl)but-3-enamide (3g):}<smiles>C=CCC(=O)N(Cc1ccc(OC)cc1)C(C(F)=Cc1ccccc1)C(C)(C)C</smiles>

$100 \mu \mathrm{mol}$, yield $37 \%$ ) as a light yellow oil.

The general procedure for the synthesis of the PMB protected bisolefins using secondary amine $7 \mathrm{~g}$ ( 1 eq., $130 \mathrm{mg}, 415 \mu \mathrm{mol})$ followed by a purification by chromatography over silica gel (eluent: pentane: $\left.\mathrm{Et}_{2} \mathrm{O}=60: 40\right)$ afforded the $(\boldsymbol{R}, \mathbf{Z})-\mathbf{N}$-(2-fluoro-4-methyl-1phenylpent-1-en-3-yl)-N-(4-methoxybenzyl)but-3-enamide (43 mg,

${ }^{\mathbf{1}} \mathbf{H}$ NMR (400.13 MHz, $\left.\mathbf{C D C l}_{3}\right): \delta(\mathrm{ppm}) 7.25$ (m, 5H, $\mathrm{H}_{1,2,3}$ both rotamers), $7.13\left(\mathrm{~d},{ }^{3} \mathrm{~J}_{\mathrm{H}-\mathrm{H}}=9 \mathrm{~Hz}, 2 \mathrm{H}\right.$, $\mathrm{H}_{10}$ both rotamers), $6.79\left(\mathrm{~d},{ }^{3} J_{\mathrm{H}-\mathrm{H}}=9 \mathrm{~Hz}, 2 \mathrm{H}, \mathrm{H}_{11}\right.$ rotamer 2$), 6.73\left(\mathrm{~d},{ }^{3} \mathrm{~J}_{\mathrm{H}-\mathrm{H}}=9 \mathrm{~Hz}, 2 \mathrm{H}, \mathrm{H}_{11}\right.$ rotamer 1$)$, $6.08\left(\mathrm{~m}, 1 \mathrm{H}, \mathrm{H}_{15}\right.$ rotamer 1), $5.95\left(\mathrm{~m}, 1 \mathrm{H}, \mathrm{H}_{15}\right.$ rotamer 2$), 5.88\left(\mathrm{~d},{ }^{3} \mathrm{~J}_{\mathrm{H}-\mathrm{F}}=39 \mathrm{~Hz}, 1 \mathrm{H}, \mathrm{H}_{5}\right.$ rotamer 2), 5.70 $\left(\mathrm{d},{ }^{3} J_{\mathrm{H}-\mathrm{F}}=39 \mathrm{~Hz}, 1 \mathrm{H}, \mathrm{H}_{5}\right.$ rotamer 1), $5.23\left(\mathrm{~m}, 2 \mathrm{H}, \mathrm{H}_{16}\right.$ rotamer 1), $5.22\left(\mathrm{~m}, 1 \mathrm{H}, \mathrm{H}_{7}\right.$ rotamer 2$), 5.07(\mathrm{~m}$, $2 \mathrm{H}, \mathrm{H}_{16}$ rotamer 2), $4.62\left(\mathrm{~m}, 2 \mathrm{H}, \mathrm{H}_{8}\right.$ both rotamers), $4.10\left(\mathrm{dd},{ }^{3} J_{\mathrm{H}-\mathrm{H}}=11 \mathrm{~Hz},{ }^{3} J_{\mathrm{H}-\mathrm{F}}=20 \mathrm{~Hz}, 1 \mathrm{H}, \mathrm{H}_{7}\right.$ rotamer 1), 3.74 (s, $3 \mathrm{H}, \mathrm{H}_{17}$ rotamer 2), 3.72 (s, $3 \mathrm{H}, \mathrm{H}_{17}$ rotamer 1), 3.35 (m, $2 \mathrm{H}, \mathrm{H}_{14}$ rotamer 1), $3.06\left(\mathrm{~m}, \mathrm{H}_{14}\right.$ rotamer 2), 2.22 (m, 1H, $\mathrm{H}_{18}$ both rotamers), 1.01 (m, $6 \mathrm{H}, \mathrm{H}_{19}$ both rotamers). ${ }^{19} \mathbf{F}$ NMR (376.50 MHz, $\left.\mathbf{C D C l}_{3}\right): \delta(\mathrm{ppm})-107.77\left(\mathrm{dd},{ }^{3} J_{\mathrm{F}-\mathrm{H}}=20 \mathrm{~Hz},{ }^{3} J_{\mathrm{F}-\mathrm{H}}=39 \mathrm{~Hz}, 1 \mathrm{~F}, \mathrm{~F}_{6}\right.$ rotamer 1$),-111.61\left(\mathrm{dd},{ }^{3} J_{\mathrm{F}-\mathrm{H}}=27 \mathrm{~Hz}\right.$, ${ }^{3} J_{\mathrm{F}-\mathrm{H}}=39 \mathrm{~Hz}, 1 \mathrm{~F}, \mathrm{~F}_{6}$ rotamer 2). ${ }^{13} \mathbf{C}$ NMR (75.47 $\left.\mathbf{M H z}, \mathbf{C D C l}_{3}\right): \delta(\mathrm{ppm}) 172.5\left(\mathrm{C}_{13}\right.$ both rotamers), $158.7\left(\mathrm{C}_{12}\right.$ rotamer 2$), 158.4\left(\mathrm{C}_{12}\right.$ rotamer 1$), 157.4\left(\mathrm{~d},{ }^{1} J_{\mathrm{C}-\mathrm{F}}=272 \mathrm{~Hz}, \mathrm{C}_{6}\right.$ both rotamers $), 132.8\left(\mathrm{~d},{ }^{3} J_{\mathrm{C}-\mathrm{F}}\right.$ $=2 \mathrm{~Hz}, \mathrm{C}_{4}$ rotamer 2$), 132.2\left(\mathrm{~d},{ }^{3} \mathrm{~J}_{\mathrm{C}-\mathrm{F}}=3 \mathrm{~Hz}, \mathrm{C}_{4}\right.$ rotamer 1$), 131.7\left(\mathrm{C}_{15}\right.$ both rotamers $), 129.6-126.9\left(\mathrm{C}_{1}\right.$, 2, 3, 9, 10 both rotamers), $118.2\left(\mathrm{C}_{16}\right.$ rotamer 1$), 117.9\left(\mathrm{C}_{16}\right.$ rotamer 2$), 114.1\left(\mathrm{C}_{11}\right.$ rotamer 2$), 113.5\left(\mathrm{C}_{11}\right.$ rotamer 1), $110.7\left(\mathrm{~d},{ }^{2} J_{\mathrm{C}-\mathrm{F}}=7 \mathrm{~Hz}, \mathrm{C}_{5}\right.$ rotamer 2$), 110.4\left(\mathrm{~d},{ }^{2} J_{\mathrm{C}-\mathrm{F}}=6 \mathrm{~Hz}, \mathrm{C}_{5}\right.$ rotamer 1$), 61.3\left(\mathrm{~d},{ }^{2} J_{\mathrm{C}-\mathrm{F}}=\right.$ $25 \mathrm{~Hz}, \mathrm{C}_{7}$ both rotamers), 55.3 ( $\mathrm{C}_{17}$ rotamer 2$), 55.2\left(\mathrm{C}_{17}\right.$ rotamer 1$), 47.2\left(\mathrm{~d},{ }^{4} J_{\mathrm{C}-\mathrm{F}}=3 \mathrm{~Hz}, \mathrm{C}_{8}\right.$ rotamer 2$)$, $45.7\left(\mathrm{~d},{ }^{4} J_{\mathrm{C}-\mathrm{F}}=3 \mathrm{~Hz}, \mathrm{C}_{8}\right.$ rotamer 1$), 39.4\left(\mathrm{C}_{14}\right.$ rotamer 1$), 39.1\left(\mathrm{C}_{14}\right.$ rotamer 2$), 26.9\left(\mathrm{~d},{ }^{3} J_{\mathrm{C}-\mathrm{F}}=2 \mathrm{~Hz}, \mathrm{C}_{18}\right.$ 
both rotamers), 20.6 (3C, $\mathrm{C}_{19}$ rotamer 1), 20.0 (3C, $\mathrm{C}_{19}$ rotamer 2), 19.5 (3C, $\mathrm{C}_{19}$ rotamer 1), 19.1 (3C, $\mathrm{C}_{19}$ rotamer 2). IR (neat) $v 2964,1646,1613,1586,1512,1464,1449,1405,1389,1289,1245,1174$, 1108, 1033, 993, 968, 915, 883, 808, 737, 693, $664 \mathrm{~cm}^{-1}$. HR-MS (ESI+): m/z = $382.2197[\mathrm{M}+\mathrm{H}]^{+}$, calcd. for $\mathrm{C}_{24} \mathrm{H}_{29} \mathrm{FNO}_{2}$ : 382.2182. [ $\left.\boldsymbol{\alpha}\right]_{\mathbf{D}}^{\mathbf{2 5}}=+181\left(c\right.$ 1.42, $\left.\mathrm{CH}_{3} \mathrm{Cl}\right)$. Chiral HPLC: Method conditions: Col. Daicel Chiralpak ASH 4.6mmx250mm $5 \mu \mathrm{m}, 1 \mathrm{~mL} / \mathrm{min}, 20^{\circ} \mathrm{C}, 60 \%$ n-heptane $40 \%$ propanol; peak $1: \operatorname{tr}=6.169 \mathrm{~min}, \%$ Area $=100 \% . R f=0.27\left(40 \% \mathrm{Et}_{2} \mathrm{O}\right.$ in pentane $)$.

Mixture of diastereoisomers: Chiral HPLC: Method conditions: Col. Daicel Chiralpak ASH $4.6 \mathrm{mmx} 250 \mathrm{~mm} 5 \mu \mathrm{m}, 1 \mathrm{~mL} / \mathrm{min}, 20^{\circ} \mathrm{C}, 60 \%$ n-heptane $40 \%$ propanol; peak 1: $\operatorname{tr}=3.762 \mathrm{~min}, \%$ Area $=$ $26.51 \%$; peak $2: \operatorname{tr}=6.150 \mathrm{~min}, \%$ Area $=73.49 \%$.

(S,Z)-N-(3-fluoro-1,4-diphenylbut-3-en-2-yl)-N-(4-methoxybenzyl)but-3-enamide (3h):

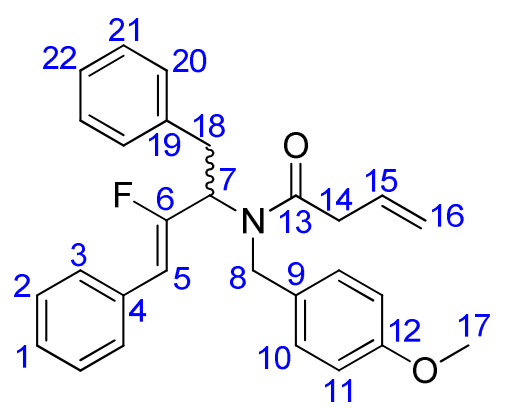

The general procedure for the synthesis of the PMB protected bisolefins using secondary amine 7 h $(1$ eq., $250 \mathrm{mg}, 692 \mu \mathrm{mol})$ followed by a purification by chromatography over silica gel (eluent: pentane: $\left.\mathrm{Et}_{2} \mathrm{O}=60: 40\right)$ afforded the (S,Z)-N-(3-fluoro-1,4diphenylbut-3-en-2-yl)-N-(4-methoxybenzyl)but-3-enamide $\quad(230$ $\mathrm{mg}, 536 \mu \mathrm{mol}$, yield $78 \%$ ) as a colorless oil.

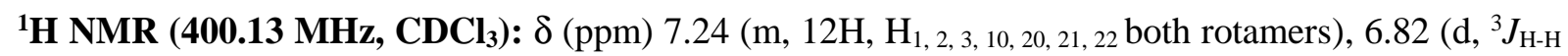
$=9 \mathrm{~Hz}, 2 \mathrm{H}, \mathrm{H}_{11}$ rotamer 2), $6.77\left(\mathrm{~d},{ }^{3} J_{\mathrm{H}-\mathrm{H}}=9 \mathrm{~Hz}, 2 \mathrm{H}, \mathrm{H}_{11}\right.$ rotamer 1$), 5.83\left(\mathrm{~m}, 1 \mathrm{H}, \mathrm{H}_{15}\right.$ both rotamers $)$, $5.75\left(\mathrm{~d},{ }^{3} \mathrm{~J}_{\mathrm{H}-\mathrm{F}}=39 \mathrm{~Hz}, 1 \mathrm{H}, \mathrm{H}_{5}\right.$ both rotamers), $5.08\left(\mathrm{~m}, 2 \mathrm{H}, \mathrm{H}_{16}\right.$ rotamer 1), $5.02\left(\mathrm{~m}, 2 \mathrm{H}, \mathrm{H}_{16}\right.$ rotamer 2), $4.67\left(\mathrm{~m}, 2 \mathrm{H}, \mathrm{H}_{8}\right.$ rotamer 1$), 4.58\left(\mathrm{~m}, 2 \mathrm{H}, \mathrm{H}_{8}\right.$ rotamer 2$), 5.79\left(\mathrm{~m}, 1 \mathrm{H}, \mathrm{H}_{7}\right.$ rotamer 2$), 4.80\left(\mathrm{~m}, 1 \mathrm{H}, \mathrm{H}_{7}\right.$ rotamer 1), 3.77 (s, $3 \mathrm{H}, \mathrm{H}_{17}$ rotamer 2), 3.75 (s, $3 \mathrm{H}, \mathrm{H}_{17}$ rotamer 1), 3.09 (m, $2 \mathrm{H}, \mathrm{H}_{18}$ both rotamers), $3.03\left(\mathrm{~d},{ }^{3} J_{\mathrm{H}-\mathrm{H}}=7 \mathrm{~Hz}, 2 \mathrm{H}, \mathrm{H}_{14}\right.$ rotamer 2$), 2.78\left(\mathrm{~m}, \mathrm{H}_{14}\right.$ rotamer 1$) .{ }^{19} \mathbf{F}$ NMR (376.50 $\left.\mathbf{M H z}, \mathbf{C D C l}_{3}\right): \delta$ $(\mathrm{ppm})-105.41\left(\mathrm{dd},{ }^{3} J_{\mathrm{F}-\mathrm{H}}=12 \mathrm{~Hz},{ }^{3} J_{\mathrm{F}-\mathrm{H}}=38 \mathrm{~Hz}, 1 \mathrm{~F}, \mathrm{~F}_{6}\right.$ rotamer 1$),-109.37\left(\mathrm{dd},{ }^{3} J_{\mathrm{F}-\mathrm{H}}=20 \mathrm{~Hz},{ }^{3} J_{\mathrm{F}-\mathrm{H}}=\right.$ $38 \mathrm{~Hz}, 1 \mathrm{~F}, \mathrm{~F}_{6}$ rotamer 2). ${ }^{13} \mathbf{C}$ NMR (75.47 $\left.\mathbf{~ M H z}, \mathbf{C D C l}_{3}\right): \delta(\mathrm{ppm}) 172.2\left(\mathrm{C}_{13}\right.$ rotamer 2$), 171.9\left(\mathrm{C}_{13}\right.$ rotamer 1$), 158.9\left(\mathrm{C}_{12}\right.$ rotamer 2$), 158.6\left(\mathrm{C}_{12}\right.$ rotamer 1$), 156.9\left(\mathrm{~d},{ }^{1} J_{\mathrm{C}-\mathrm{F}}=269 \mathrm{~Hz}, \mathrm{C}_{6}\right.$ rotamer 2$), 155.4$ $\left(\mathrm{d},{ }^{1} J_{\mathrm{C}-\mathrm{F}}=270 \mathrm{~Hz}, \mathrm{C}_{6}\right.$ rotamer 1$), 136.9\left(\mathrm{C}_{15}\right.$ rotamer 2$), 136.7\left(\mathrm{C}_{15}\right.$ rotamer 1$), 132.7\left(\mathrm{~d},{ }^{3} J_{\mathrm{C}-\mathrm{F}}=2 \mathrm{~Hz}, \mathrm{C}_{4}\right.$ rotamer 2), $132.1\left(\mathrm{~d},{ }^{3} J_{\mathrm{C}-\mathrm{F}}=3 \mathrm{~Hz}, \mathrm{C}_{4}\right.$ rotamer 1$), 131.6\left(\mathrm{C}_{19}\right.$ rotamer 1$), 131.5\left(\mathrm{C}_{19}\right.$ rotamer 2$), 129.7$ $126.8\left(\mathrm{C}_{1,2,3,9,10,20,21,22}\right.$ both rotamers $), 118.0\left(\mathrm{C}_{16}\right.$ rotamer 1$), 117.9\left(\mathrm{C}_{16}\right.$ rotamer 2$), 114.2\left(\mathrm{C}_{11}\right.$ rotamer 2), $113.7\left(\mathrm{C}_{11}\right.$ rotamer 1$), 110.5\left(\mathrm{~d},{ }^{2} J_{\mathrm{C}-\mathrm{F}}=7 \mathrm{~Hz}, \mathrm{C}_{5}\right.$ rotamer 2$), 109.9\left(\mathrm{~d},{ }^{2} J_{\mathrm{C}-\mathrm{F}}=7 \mathrm{~Hz}, \mathrm{C}_{5}\right.$ rotamer 1$), 58.5$ $\left(\mathrm{d},{ }^{2} J_{\mathrm{C}-\mathrm{F}}=25 \mathrm{~Hz}, \mathrm{C}_{7}\right.$ rotamer 1$), 56.7\left(\mathrm{~d},{ }^{2} J_{\mathrm{C}-\mathrm{F}}=25 \mathrm{~Hz}, \mathrm{C}_{7}\right.$ rotamer 2$), 55.3\left(\mathrm{C}_{17}\right.$ rotamer 2$), 55.2\left(\mathrm{C}_{17}\right.$ 
rotamer 1$), 47.8\left(\mathrm{C}_{8}\right.$ rotamer 2$), 45.4\left(\mathrm{C}_{8}\right.$ rotamer 1$), 39.2\left(\mathrm{C}_{14}\right.$ rotamer 2$), 38.6\left(\mathrm{C}_{14}\right.$ rotamer 1$), 36.4(\mathrm{~d}$, ${ }^{3} J_{\mathrm{C}-\mathrm{F}}=3 \mathrm{~Hz}, \mathrm{C}_{18}$ rotamer 1$), 35.8\left(\mathrm{~d},{ }^{3} J_{\mathrm{C}-\mathrm{F}}=3 \mathrm{~Hz}, \mathrm{C}_{18}\right.$ rotamer 2$)$. IR (neat) $v 3062,3028,2933,2836$, 1686, 1646, 1612, 1585, 1511, 1495, 1449, 1404, 1357, 1328, 1302, 1291, 1245, 1175, 1108, 1079, 1031, 993, 959, 915, 822, 749, 733, $693 \mathrm{~cm}^{-1}$. HR-MS (ESI+): $\mathrm{m} / \mathrm{z}=430.2194[\mathrm{M}+\mathrm{H}]^{+}$, calcd. for $\mathrm{C}_{28} \mathrm{H}_{29} \mathrm{FNO}_{2}: 430.2182 . R f=0.35\left(50 \% \mathrm{Et}_{2} \mathrm{O}\right.$ in pentane $)$.

\section{f. Synthesis of the cyclic lactams 2 through ring-closing-metathesis}

\section{General procedure:}

An oven-dried microwave flask equipped with a stir bar was filled under argon with diene (1eq.) and perfluorotoluene $(0.02 \mathrm{M})$ before the addition of the pre-catalyst $\mathbf{M 2}$ (reported number of eq.). The mixture was heated at the reported temperature during 15 minutes into a microwave reactor at $200 \mathrm{~W}$. The vial content was then transferred into a round-bottom flask from the which the solvent was evaporated. The conversion was calculated by both ${ }^{1} \mathrm{H}$ and ${ }^{19} \mathrm{~F}$ NMR integrations. A purification was then carried out by flash chromatography on silica gel $\left(\mathrm{Pentane}_{\mathrm{Et}} \mathrm{O}\right)$ to reach the desired cyclic lactam.

(S)-5-fluoro-1-(4-methoxybenzyl)-6-methyl-1,6-dihydropyridin-2(3H)-one (2b):

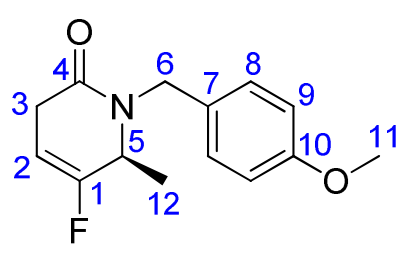

The general procedure for the synthesis of the cyclic lactams through ringclosing-metathesis using PMB-protected bisolefin 3b (1 eq., 26 mg, 74 $\mu \mathrm{mol}$ ) and $\mathrm{M} 2$ catalyst (2 eq.) at $70^{\circ} \mathrm{C}$ followed by purification by chromatography on silica gel (Pentane: $\mathrm{Et}_{2} \mathrm{O}=60: 40$ ) afforded the $(S)$-5fluoro-1-(4-methoxybenzyl)-6-methyl-1,6-dihydropyridin-2(3H)-one (14.3 mg, 66 umol, yield $95 \%)$ as a thick light brown oil.

${ }^{1} \mathbf{H}$ NMR (400.13 MHz, CDCl 3 ): $\delta(\mathrm{ppm}) 7.18\left(\mathrm{~d},{ }^{3} J_{\mathrm{H}-\mathrm{H}}=9 \mathrm{~Hz}, 2 \mathrm{H}, \mathrm{H}_{8}\right), 6.86\left(\mathrm{~d},{ }^{3} J_{\mathrm{H}-\mathrm{H}}=9 \mathrm{~Hz}, 2 \mathrm{H}, \mathrm{H}_{9}\right)$, $5.39\left(\mathrm{~d},{ }^{2} J_{\mathrm{H}-\mathrm{H}}=15 \mathrm{~Hz}, 1 \mathrm{H}, \mathrm{H}_{6}\right), 5.22\left(\mathrm{~m}, 1 \mathrm{H}, \mathrm{H}_{2}\right), 3.90\left(\mathrm{~d},{ }^{2} J_{\mathrm{H}-\mathrm{H}}=15 \mathrm{~Hz}, 1 \mathrm{H}, \mathrm{H}_{6}\right), 3.83\left(\mathrm{~m}, 1 \mathrm{H}, \mathrm{H}_{5}\right), 3.79$ $\left(\mathrm{s}, 3 \mathrm{H}, \mathrm{H}_{11}\right), 3.10\left(\mathrm{~m}, 2 \mathrm{H}, \mathrm{H}_{3}\right), 1.39\left(\mathrm{~d},{ }^{3} \mathrm{~J}_{\mathrm{H}-\mathrm{H}}=7 \mathrm{~Hz}, 3 \mathrm{H}, \mathrm{H}_{12}\right) .{ }^{19} \mathbf{F}$ NMR $\left(376.50 \mathbf{M H z}, \mathbf{C D C l}_{3}\right): \delta(\mathrm{ppm})$ -116.88 (m, 1F, F $\left.{ }_{1}\right) .{ }^{13} \mathbf{C}$ NMR (75.47 MHz, $\left.\mathbf{C D C l}_{3}\right): \delta(\mathrm{ppm}) 166.8\left(\mathrm{~s}, 1 \mathrm{C}, \mathrm{C}_{4}\right), 159.2\left(\mathrm{~s}, 1 \mathrm{C}, \mathrm{C}_{10}\right), 156.2$ $\left(\mathrm{d},{ }^{1} J_{\mathrm{C}-\mathrm{F}}=254 \mathrm{~Hz}, 1 \mathrm{C}, \mathrm{C}_{1}\right), 129.4\left(\mathrm{~s}, 2 \mathrm{C}, \mathrm{C}_{8}\right), 128.4\left(1 \mathrm{C}, \mathrm{C}_{7}\right), 114.2\left(\mathrm{~s}, 2 \mathrm{C}, \mathrm{C}_{9}\right), 96.9\left(\mathrm{~d},{ }^{2} J_{\mathrm{C}-\mathrm{F}}=17 \mathrm{~Hz}, 1 \mathrm{C}\right.$, 
$\left.\mathrm{C}_{2}\right), 55.3\left(\mathrm{~s}, 1 \mathrm{C}, \mathrm{C}_{11}\right), 51.0\left(\mathrm{~d},{ }^{2} J_{\mathrm{C}-\mathrm{F}}=36 \mathrm{~Hz}, 1 \mathrm{C}, \mathrm{C}_{5}\right), 45.9\left(\mathrm{~s}, 1 \mathrm{C}, \mathrm{C}_{6}\right), 29.9\left(\mathrm{~d},{ }^{3} J_{\mathrm{C}-\mathrm{F}}=9 \mathrm{~Hz}, 1 \mathrm{C}, \mathrm{C}_{3}\right), 18.3$ $\left(\mathrm{d},{ }^{2} J_{\mathrm{C}-\mathrm{F}}=2 \mathrm{~Hz}, 1 \mathrm{C}, \mathrm{C}_{12}\right)$. IR (neat) $v 2934,2837,1729,1644,1613,1585,1512,1462,1414,1379,1357$, 1303, 1282, 1241, 1171, 1083, 1113, 1067, 1027, 983, 905, 813, 752, 721, $693 \mathrm{~cm}^{-1}$. HR-MS (ESI+): $\mathrm{m} / \mathrm{z}=250.1254[\mathrm{M}+\mathrm{H}]^{+}$, calcd. for $\mathrm{C}_{14} \mathrm{H}_{17} \mathrm{FNO}_{2}:$ 250.1243. [ $\left.\alpha\right]_{\mathbf{D}}^{25}=-106\left(c 1.5, \mathrm{CH}_{3} \mathrm{Cl}\right)$. Chiral HPLC: Method conditions: Col. Daicel Chiralpak ASH 4.6mmx250mm $5 \mu \mathrm{m}, 1 \mathrm{~mL} / \mathrm{min}, 20^{\circ} \mathrm{C}, 80 \% \mathrm{n}$-heptane $20 \%$ propanol; peak $1: \operatorname{tr}=8.360 \mathrm{~min}, \%$ Area $=98.97 \%$; peak $2: \operatorname{tr}=9.962 \mathrm{~min}, \%$ Area $=1.03 \%$. $R f=$ $0.23\left(50 \% \mathrm{Et}_{2} \mathrm{O}\right.$ in pentane).

(R)-5-fluoro-1-(4-methoxybenzyl)-6-methyl-1,6-dihydropyridin-2(3H)-one (2c):

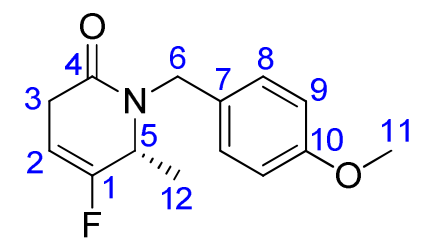

The general procedure for the synthesis of the cyclic lactams through ringclosing-metathesis using PMB-protected bisolefin 3c (1 eq., $18 \mathrm{mg}, 51$ $\mu \mathrm{mol}$ ) and $\mathrm{M} 2$ catalyst (2 eq.) at $70^{\circ} \mathrm{C}$ followed by purification by chromatography on silica gel (Pentane: $\mathrm{Et}_{2} \mathrm{O}=60: 40$ ) afforded the $(\boldsymbol{R})$-5fluoro-1-(4-methoxybenzyl)-6-methyl-1,6-dihydropyridin-2(3H)-one (9.9 mg, $46 \mu \mathrm{mol}$, yield $91 \%$ ) as a thick light brown oil.

${ }^{1} \mathbf{H}$ NMR (400.13 MHz, CDCl 3 ): $\delta(\mathrm{ppm}) 7.18\left(\mathrm{~d},{ }^{3} J_{\mathrm{H}-\mathrm{H}}=9 \mathrm{~Hz}, 2 \mathrm{H}, \mathrm{H}_{8}\right), 6.86\left(\mathrm{~d},{ }^{3} J_{\mathrm{H}-\mathrm{H}}=9 \mathrm{~Hz}, 2 \mathrm{H}, \mathrm{H}_{9}\right)$, $5.39\left(\mathrm{~d},{ }^{2} J_{\mathrm{H}-\mathrm{H}}=15 \mathrm{~Hz}, 1 \mathrm{H}, \mathrm{H}_{6}\right), 5.23\left(\mathrm{~m}, 1 \mathrm{H}, \mathrm{H}_{2}\right), 3.90\left(\mathrm{~d},{ }^{2} J_{\mathrm{H}-\mathrm{H}}=15 \mathrm{~Hz}, 1 \mathrm{H}, \mathrm{H}_{6}\right), 3.83\left(\mathrm{~m}, 1 \mathrm{H}, \mathrm{H}_{5}\right), 3.80$ $\left(\mathrm{s}, 3 \mathrm{H}, \mathrm{H}_{11}\right), 3.10\left(\mathrm{~m}, 2 \mathrm{H}, \mathrm{H}_{3}\right), 1.39\left(\mathrm{~d},{ }^{3} \mathrm{~J}_{\mathrm{H}-\mathrm{H}}=7 \mathrm{~Hz}, 3 \mathrm{H}, \mathrm{H}_{12}\right) .{ }^{19} \mathbf{F}$ NMR $\left(\mathbf{3 7 6 . 5 0} \mathbf{M H z}, \mathbf{C D C l}_{3}\right): \delta(\mathrm{ppm})$ $-116.88\left(\mathrm{~m}, 1 \mathrm{~F}, \mathrm{~F}_{1}\right) .{ }^{13} \mathbf{C}$ NMR (75.47 MHz, $\left.\mathbf{C D C l}_{3}\right): \delta(\mathrm{ppm}) 166.8\left(\mathrm{~s}, 1 \mathrm{C}, \mathrm{C}_{4}\right), 159.2\left(\mathrm{~s}, 1 \mathrm{C}, \mathrm{C}_{10}\right), 156.2$ $\left(\mathrm{d},{ }^{1} J_{\mathrm{C}-\mathrm{F}}=254 \mathrm{~Hz}, 1 \mathrm{C}, \mathrm{C}_{1}\right), 129.4\left(\mathrm{~s}, 2 \mathrm{C}, \mathrm{C}_{8}\right), 128.4\left(1 \mathrm{C}, \mathrm{C}_{7}\right), 114.2\left(\mathrm{~s}, 2 \mathrm{C}, \mathrm{C}_{9}\right), 96.9\left(\mathrm{~d},{ }^{2} J_{\mathrm{C}-\mathrm{F}}=17 \mathrm{~Hz}, 1 \mathrm{C}\right.$, $\left.\mathrm{C}_{2}\right), 55.3\left(\mathrm{~s}, 1 \mathrm{C}, \mathrm{C}_{11}\right), 51.0\left(\mathrm{~d},{ }^{2} J_{\mathrm{C}-\mathrm{F}}=36 \mathrm{~Hz}, 1 \mathrm{C}, \mathrm{C}_{5}\right), 45.9\left(\mathrm{~s}, 1 \mathrm{C}, \mathrm{C}_{6}\right), 29.9\left(\mathrm{~d},{ }^{3} J_{\mathrm{C}-\mathrm{F}}=9 \mathrm{~Hz}, 1 \mathrm{C}, \mathrm{C}_{3}\right), 18.3$ $\left(\mathrm{d},{ }^{2} J_{\mathrm{C}-\mathrm{F}}=2 \mathrm{~Hz}, 1 \mathrm{C}, \mathrm{C}_{12}\right)$. IR (neat) $v 2936,2837,1729,1644,1613,1585,1512,1463,1414,1379,1357$, 1304, 1283, 1243, 1172, 1113, 1067, 1028, 983, 906, 814, 752, 721, $693 \mathrm{~cm}^{-1}$. HR-MS (ESI+): m/z = $250.1255[\mathrm{M}+\mathrm{H}]^{+}$, calcd. for $\mathrm{C}_{14} \mathrm{H}_{17} \mathrm{FNO}_{2}:$ 250.1243. $[\boldsymbol{\alpha}]_{\mathrm{D}}^{25}=+97(c$ 1.3, $\mathrm{CH} 3 \mathrm{Cl})$. Chiral HPLC: Method conditions: Col. Daicel Chiralpak ASH 4.6mmx250mm $5 \mu \mathrm{m}, 1 \mathrm{~mL} / \mathrm{min}, 20^{\circ} \mathrm{C}, 80 \% \mathrm{n}$-heptane $20 \%$ propanol; peak $1: \operatorname{tr}=8.370 \mathrm{~min}, \%$ Area $=0.57 \%$; peak $2: \operatorname{tr}=9.929 \mathrm{~min}, \%$ Area $=99.43 \% . R f=$ $0.23\left(50 \% \mathrm{Et}_{2} \mathrm{O}\right.$ in pentane).

Racemic mixture: Chiral HPLC: Method conditions: Col. Daicel Chiralpak ASH 4.6mmx250mm $5 \mu \mathrm{m}, 1 \mathrm{~mL} / \mathrm{min}, 20^{\circ} \mathrm{C}, 80 \% \mathrm{n}$-heptane $20 \%$ propanol; peak $1: \operatorname{tr}=8.349 \mathrm{~min}, \%$ Area $=47.88 \%$; peak $2: \operatorname{tr}=9.928 \mathrm{~min}, \%$ Area $=52.12 \%$.

(S)-5-fluoro-1-(4-methoxybenzyl)-6-phenyl-1,6-dihydropyridin-2(3H)-one (2d): 


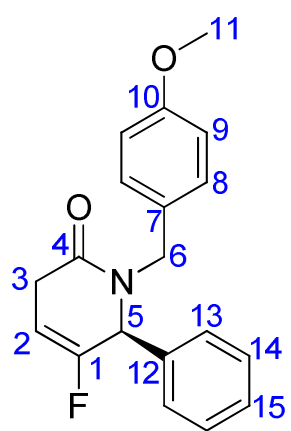

The general procedure for the synthesis of the cyclic lactams through ring-closingmetathesis using PMB-protected bisolefin 3d (1 eq., $215 \mathrm{mg}, 518 \mu \mathrm{mol})$ and M2 catalyst ( 2 eq.) at $70^{\circ} \mathrm{C}$ followed by purification by chromatography on silica gel (Pentane: $\mathrm{Et}_{2} \mathrm{O}=60: 40$ ) afforded the (S)-5-fluoro-1-(4-methoxybenzyl)-6-phenyl1,6-dihydropyridin-2(3H)-one (110 mg, $511 \mu \mathrm{mol}$, yield $98 \%)$ as a thick light brown oil.

${ }^{1} \mathbf{H}$ NMR (400.13 MHz, CDCl $)$ : $\delta(\mathrm{ppm}) 7.41\left(\mathrm{~m}, 3 \mathrm{H}, \mathrm{H}_{14,15}\right), 7.25\left(\mathrm{~m}, 2 \mathrm{H}, \mathrm{H}_{13}\right), 7.12\left(\mathrm{~d},{ }^{3} J_{\mathrm{H}-\mathrm{H}}=8 \mathrm{~Hz}\right.$, $\left.2 \mathrm{H}, \mathrm{H}_{8}\right), 6.87\left(\mathrm{~d},{ }^{3} J_{\mathrm{H}-\mathrm{H}}=8 \mathrm{~Hz}, 2 \mathrm{H}, \mathrm{H}_{9}\right), 5.50\left(\mathrm{dd},{ }^{4} J_{\mathrm{H}-\mathrm{H}}=2 \mathrm{~Hz},{ }^{2} J_{\mathrm{H}-\mathrm{H}}=14 \mathrm{~Hz}, 1 \mathrm{H}, \mathrm{H}_{6}\right), 5.27\left(\mathrm{~m}, 1 \mathrm{H}, \mathrm{H}_{2}\right)$, $4.73\left(\mathrm{~d},{ }^{3} J_{\mathrm{H}-\mathrm{F}}=7 \mathrm{~Hz}, 1 \mathrm{H}, \mathrm{H}_{5}\right), 3.81\left(\mathrm{~s}, 3 \mathrm{H}, \mathrm{H}_{11}\right), 3.29\left(\mathrm{~m}, 2 \mathrm{H}, \mathrm{H}_{3}\right), 3.29\left(\mathrm{~d},{ }^{2} J_{\mathrm{H}-\mathrm{H}}=14 \mathrm{~Hz}, 1 \mathrm{H}, \mathrm{H}_{6}\right) .{ }^{19} \mathbf{F}$ NMR (376.50 MHz, CDCl $\left.\mathbf{C l}_{3}\right): \delta(\mathrm{ppm})-115.13\left(\mathrm{~m}, 1 \mathrm{~F}, \mathrm{~F}_{1}\right) .{ }^{13} \mathrm{C}$ NMR (75.47 MHz, $\left.\mathbf{C D C l}_{3}\right): \delta(\mathrm{ppm})$ $166.6\left(\mathrm{~s}, 1 \mathrm{C}, \mathrm{C}_{4}\right), 159.3\left(\mathrm{~s}, 1 \mathrm{C}, \mathrm{C}_{10}\right), 154.1\left(\mathrm{~d},{ }^{1} J_{\mathrm{C}-\mathrm{F}}=253 \mathrm{~Hz}, 1 \mathrm{C}, \mathrm{C}_{1}\right), 137.2\left(\mathrm{~d},{ }^{3} J_{\mathrm{C}-\mathrm{F}}=2 \mathrm{~Hz}, 1 \mathrm{C}, \mathrm{C}_{12}\right)$, 129.9 (s, 2C, $\left.\mathrm{C}_{8}\right), 129.2\left(\mathrm{~s}, 2 \mathrm{C}, \mathrm{C}_{14}\right), 129.0$ (s, 1C, $\mathrm{C}_{15}$ ), 128.0 (1C, $\left.\mathrm{C}_{7}\right), 127.6$ (s, 2C, $\left.\mathrm{C}_{13}\right), 114.2$ (s, 2C, $\left.\mathrm{C}_{9}\right), 97.3\left(\mathrm{~d},{ }^{2} J_{\mathrm{C}-\mathrm{F}}=17 \mathrm{~Hz}, 1 \mathrm{C}, \mathrm{C}_{2}\right), 59.6\left(\mathrm{~d},{ }^{2} J_{\mathrm{C}-\mathrm{F}}=36 \mathrm{~Hz}, 1 \mathrm{C}, \mathrm{C}_{5}\right), 55.3\left(\mathrm{~s}, 1 \mathrm{C}, \mathrm{C}_{11}\right), 45.7\left(\mathrm{~s}, 1 \mathrm{C}, \mathrm{C}_{6}\right), 30.0$ $\left(\mathrm{d},{ }^{3} J_{\mathrm{C}-\mathrm{F}}=8 \mathrm{~Hz}, 1 \mathrm{C}, \mathrm{C}_{3}\right)$. IR (neat) $v 2933,2837,2299,1728,1646,1611,1585,1511,1493,1450,1411$, 1357, 1323, 1302, 1282, 1237, 1171, 1110, 1066, 1030, 950, 926, 834, 817, 752, 729, 698, $664 \mathrm{~cm}^{-1}$. HR-MS (ESI+): $\mathrm{m} / \mathrm{z}=312.1414[\mathrm{M}+\mathrm{H}]^{+}$, calcd. for $\mathrm{C}_{19} \mathrm{H}_{19} \mathrm{FNO}_{2}: 312.1400$. $[\boldsymbol{\alpha}]_{\mathbf{D}}^{25}=+153(c 0.85$, $\mathrm{CH}_{3} \mathrm{Cl}$ ). Chiral HPLC: Method conditions: Col. Daicel Chiralpak ASH 4.6mmx250mm $5 \mu \mathrm{m}$, $1 \mathrm{~mL} / \mathrm{min}, 20^{\circ} \mathrm{C}, 60 \% \mathrm{n}$-heptane $40 \%$ propanol; peak $1: \operatorname{tr}=23.146 \mathrm{~min}, \%$ Area $=100 \% \cdot R f=0.26(50 \%$ $\mathrm{Et}_{2} \mathrm{O}$ in pentane).

Racemic 5-fluoro-1-(4-methoxybenzyl)-6-phenyl-1,6-dihydropyridin-2(3H)-one (2d+e):

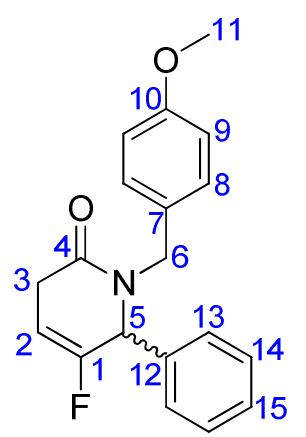

The general procedure for the synthesis of the cyclic lactams through ring-closingmetathesis using PMB-protected bisolefin 3d+e (1 eq., $55 \mathrm{mg}, 132 \mu \mathrm{mol})$ and M2 catalyst ( 2 eq.) at $70^{\circ} \mathrm{C}$ followed by purification by chromatography on silica gel (Pentane:Et ${ }_{2} \mathrm{O}=60: 40$ ) afforded the Racemic 5-fluoro-1-(4-methoxybenzyl)-6phenyl-1,6-dihydropyridin-2(3H)-one (26 mg, 121 mmol, yield $93 \%)$ as a thick light brown oil.

${ }^{1}$ H NMR (400.13 MHz, CDCl $\left.)_{3}\right): \delta(\mathrm{ppm}) 7.40\left(\mathrm{~m}, 3 \mathrm{H}, \mathrm{H}_{14,15}\right), 7.25\left(\mathrm{~m}, 2 \mathrm{H}, \mathrm{H}_{13}\right), 7.12\left(\mathrm{~d},{ }^{3} \mathrm{~J}_{\mathrm{H}-\mathrm{H}}=8 \mathrm{~Hz}\right.$, $\left.2 \mathrm{H}, \mathrm{H}_{8}\right), 6.87\left(\mathrm{~d},{ }^{3} J_{\mathrm{H}-\mathrm{H}}=8 \mathrm{~Hz}, 2 \mathrm{H}, \mathrm{H}_{9}\right), 5.49\left(\mathrm{dd},{ }^{4} J_{\mathrm{H}-\mathrm{H}}=2 \mathrm{~Hz},{ }^{2} J_{\mathrm{H}-\mathrm{H}}=14 \mathrm{~Hz}, 1 \mathrm{H}, \mathrm{H}_{6}\right), 5.27\left(\mathrm{~m}, 1 \mathrm{H}, \mathrm{H}_{2}\right)$, $4.73\left(\mathrm{~d},{ }^{3} J_{\mathrm{H}-\mathrm{F}}=7 \mathrm{~Hz}, 1 \mathrm{H}, \mathrm{H}_{5}\right), 3.81\left(\mathrm{~s}, 3 \mathrm{H}, \mathrm{H}_{11}\right), 3.30\left(\mathrm{~m}, 2 \mathrm{H}, \mathrm{H}_{3}\right), 3.30\left(\mathrm{~d},{ }^{2} \boldsymbol{J}_{\mathrm{H}-\mathrm{H}}=14 \mathrm{~Hz}, 1 \mathrm{H}, \mathrm{H}_{6}\right) .{ }^{19} \mathbf{F}$ 
NMR (376.50 MHz, CDCl $)$ : $\delta(\mathrm{ppm})-115.12\left(\mathrm{~m}, 1 \mathrm{~F}, \mathrm{~F}_{1}\right) .{ }^{13} \mathrm{C}$ NMR (75.47 MHz, $\left.\mathbf{C D C l}_{3}\right): \delta(\mathrm{ppm})$ $166.5\left(\mathrm{~s}, 1 \mathrm{C}, \mathrm{C}_{4}\right), 159.2\left(\mathrm{~s}, 1 \mathrm{C}, \mathrm{C}_{10}\right), 154.0\left(\mathrm{~d},{ }^{1} J_{\mathrm{C}-\mathrm{F}}=253 \mathrm{~Hz}, 1 \mathrm{C}, \mathrm{C}_{1}\right), 137.1\left(\mathrm{~d},{ }^{3} J_{\mathrm{C}-\mathrm{F}}=2 \mathrm{~Hz}, 1 \mathrm{C}, \mathrm{C}_{12}\right)$, 129.9 (s, 2C, $\left.\mathrm{C}_{8}\right), 129.2$ (s, 2C, $\left.\mathrm{C}_{14}\right), 129.0$ (s, 1C, $\mathrm{C}_{15}$ ), 128.0 (1C, $\left.\mathrm{C}_{7}\right), 127.6$ (s, 2C, $\left.\mathrm{C}_{13}\right), 114.1$ (s, 2C, $\left.\mathrm{C}_{9}\right), 97.2\left(\mathrm{~d},{ }^{2} J_{\mathrm{C}-\mathrm{F}}=17 \mathrm{~Hz}, 1 \mathrm{C}, \mathrm{C}_{2}\right), 59.6\left(\mathrm{~d},{ }^{2} J_{\mathrm{C}-\mathrm{F}}=36 \mathrm{~Hz}, 1 \mathrm{C}, \mathrm{C}_{5}\right), 55.3\left(\mathrm{~s}, 1 \mathrm{C}, \mathrm{C}_{11}\right), 45.7\left(\mathrm{~s}, 1 \mathrm{C}, \mathrm{C}_{6}\right), 30.0$ $\left(\mathrm{d},{ }^{3} J_{\mathrm{C}-\mathrm{F}}=8 \mathrm{~Hz}, 1 \mathrm{C}, \mathrm{C}_{3}\right.$ ). IR (neat) $v 2935,2837,2311,1729,1645,1611,1586,1511,1493,1451,1411$, 1357, 1323, 1302, 1281, 1238, 1172, 1110, 1066, 1031, 950, 927, 834, 817, 752, 729, 698, $664 \mathrm{~cm}^{-1}$. HR-MS (ESI+): $\mathrm{m} / \mathrm{z}=312.1413[\mathrm{M}+\mathrm{H}]^{+}$, calcd. for $\mathrm{C}_{19} \mathrm{H}_{19} \mathrm{FNO}_{2}: 312.1400$. Chiral HPLC: Method conditions: Col. Daicel Chiralpak ASH 4.6mmx250mm $5 \mu \mathrm{m}, 1 \mathrm{~mL} / \mathrm{min}, 20^{\circ} \mathrm{C}, 60 \%$ n-heptane $40 \%$ propanol; peak $1: \operatorname{tr}=11.805 \mathrm{~min}, \%$ Area $=50.18 \%$; peak $2: \operatorname{tr}=23.138 \mathrm{~min}, \%$ Area $=49.82 \% . R f=$ $0.26\left(50 \% \mathrm{Et}_{2} \mathrm{O}\right.$ in pentane).

\section{6-benzyl-5-fluoro-1-(4-methoxybenzyl)-1,6-dihydropyridin-2(3H)-one (2h):}

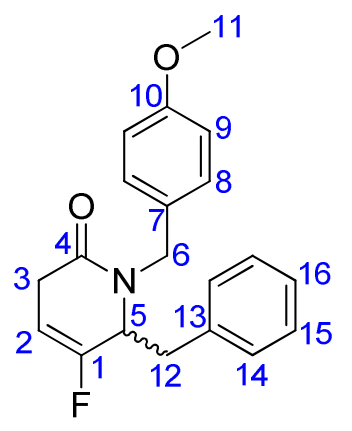

The general procedure for the synthesis of the cyclic lactams through ringclosing-metathesis using PMB-protected bisolefin $\mathbf{3 h}$ ( 1 eq., $35 \mathrm{mg}, 82 \mu \mathrm{mol}$ ) and $\mathrm{M} 2$ catalyst ( 4 eq.) at $120^{\circ} \mathrm{C}$ followed by purification by chromatography on silica gel (Pentane:Et $\mathrm{t}_{2} \mathrm{O}=70: 30$ ) afforded the 6-benzyl-5-fluoro-1-(4methoxybenzyl)-1,6-dihydropyridin-2(3H)-one (20 mg, $62 \mu \mathrm{mol}$, yield $86 \%$ ) as a thick light brown oil.

${ }^{1} \mathbf{H}$ NMR (400.13 MHz, $\left.\mathbf{C D C l}_{3}\right): \delta(\mathrm{ppm}) 7.27\left(\mathrm{~m}, 3 \mathrm{H}, \mathrm{H}_{15}, 16\right), 7.21\left(\mathrm{~d},{ }^{3} J_{\mathrm{H}-\mathrm{H}}=8 \mathrm{~Hz}, 2 \mathrm{H}, \mathrm{H}_{8}\right), 7.09(\mathrm{~m}$, $\left.2 \mathrm{H}, \mathrm{H}_{14}\right), 6.88\left(\mathrm{~d},{ }^{3} J_{\mathrm{H}-\mathrm{H}}=8 \mathrm{~Hz}, 2 \mathrm{H}, \mathrm{H}_{9}\right), 5.60\left(\mathrm{~d},{ }^{2} J_{\mathrm{H}-\mathrm{H}}=15 \mathrm{~Hz}, 1 \mathrm{H}, \mathrm{H}_{6}\right), 5.12\left(\mathrm{~m}, 1 \mathrm{H}, \mathrm{H}_{2}\right), 4.09(\mathrm{~m}, 1 \mathrm{H}$, $\left.\mathrm{H}_{5}\right), 3.88\left(\mathrm{~d},{ }^{2} J_{\mathrm{H}-\mathrm{H}}=14 \mathrm{~Hz}, 1 \mathrm{H}, \mathrm{H}_{6}\right), 3.81\left(\mathrm{~s}, 3 \mathrm{H}, \mathrm{H}_{11}\right), 3.18\left(\mathrm{dd},{ }^{3} J_{\mathrm{H}-\mathrm{H}}=4 \mathrm{~Hz},{ }^{2} J_{\mathrm{H}-\mathrm{H}}=14 \mathrm{~Hz}, 1 \mathrm{H}, \mathrm{H}_{12}\right), 2.88$ $\left(\mathrm{dd},{ }^{3} J_{\mathrm{H}-\mathrm{H}}=3 \mathrm{~Hz},{ }^{2} J_{\mathrm{H}-\mathrm{H}}=14 \mathrm{~Hz}, 1 \mathrm{H}, \mathrm{H}_{12}\right), 2.64\left(\mathrm{~m}, 1 \mathrm{H}, \mathrm{H}_{3}\right), 1.88\left(\mathrm{~m}, 1 \mathrm{H}, \mathrm{H}_{3}\right) .{ }^{19} \mathbf{F}$ NMR (376.50 MHz, $\left.\mathbf{C D C l}_{3}\right): \delta(\mathrm{ppm})-116.52\left(\mathrm{~m}, 1 \mathrm{~F}, \mathrm{~F}_{1}\right) .{ }^{13} \mathbf{C} \mathbf{N M R}\left(\mathbf{7 5 . 4 7} \mathbf{M H z}, \mathbf{C D C l}_{3}\right): \delta(\mathrm{ppm}) 167.9\left(\mathrm{~s}, 1 \mathrm{C}, \mathrm{C}_{4}\right), 159.3$ $\left(\mathrm{s}, 1 \mathrm{C}, \mathrm{C}_{10}\right), 153.5\left(\mathrm{~d},{ }^{1} J_{\mathrm{C}-\mathrm{F}}=254 \mathrm{~Hz}, 1 \mathrm{C}, \mathrm{C}_{1}\right), 134.3\left(\mathrm{~s}, 1 \mathrm{C}, \mathrm{C}_{13}\right), 130.2\left(\mathrm{~s}, 2 \mathrm{C}, \mathrm{C}_{8}\right), 129.6\left(\mathrm{~s}, 2 \mathrm{C}, \mathrm{C}_{15}\right)$, 128.3 (s, 2C, $\left.\mathrm{C}_{14}\right), 128.2\left(1 \mathrm{C}, \mathrm{C}_{7}\right), 127.3$ (s, 1C, $\left.\mathrm{C}_{16}\right), 114.3\left(\mathrm{~s}, 2 \mathrm{C}, \mathrm{C}_{9}\right), 99.8\left(\mathrm{~d},{ }^{2} J_{\mathrm{C}-\mathrm{F}}=16 \mathrm{~Hz}, 1 \mathrm{C}, \mathrm{C}_{2}\right)$, $56.0\left(\mathrm{~d},{ }^{2} J_{\mathrm{C}-\mathrm{F}}=36 \mathrm{~Hz}, 1 \mathrm{C}, \mathrm{C}_{5}\right), 55.3\left(\mathrm{~s}, 1 \mathrm{C}, \mathrm{C}_{11}\right), 46.0\left(\mathrm{~s}, 1 \mathrm{C}, \mathrm{C}_{6}\right), 35.5\left(\mathrm{~s}, 1 \mathrm{C}, \mathrm{C}_{12}\right), 29.5\left(\mathrm{~d},{ }^{3} J_{\mathrm{C}-\mathrm{F}}=9 \mathrm{~Hz}\right.$, $1 \mathrm{C}, \mathrm{C}_{3}$ ). IR (neat) $v$ 3063, 3030, 2934, 2837, 1727, 1645, 1612, 1585, 1511, 1496, 1455, 1413, 1384, 1357, 1318, 1303, 1284, 1243, 1172, 1110, 1070, 1032, 970, 904, 815, 753, 725, 702, $666 \mathrm{~cm}^{-1}$. HRMS (ESI+): $\mathrm{m} / \mathrm{z}=326.1550[\mathrm{M}+\mathrm{H}]^{+}$, calcd. for $\mathrm{C}_{20} \mathrm{H}_{21} \mathrm{FNO}_{2}: 326.1556 . R f=0.20\left(50 \% \mathrm{Et}_{2} \mathrm{O}\right.$ in pentane).

IV. Synthesis of the various fluoropseudopeptides $\mathbf{1}$ 
1. Experimental procedures and datas

a. Synthesis of the deprotected lactams $\mathbf{1 0}$

\section{General procedure for the PMB-deprotection of the lactams:}

In an oven dried round-bottom flask equipped with a stir bar and flushed with argon, a solution of the starting lactam (1 eq.), in $\mathrm{MeCN}: \mathrm{H}_{2} \mathrm{O}$ (5:1 v/v) mixture was prepared. The CAN (5 eq.) powder was added portionwise to the mixture. The resulting solution was stirred at room temperature and the progress of the reaction was controlled by TLC and ${ }^{19} \mathrm{~F}$ NMR. When full consumption of starting material was observed, the reaction was stopped by adding a saturated solution of $\mathrm{NaHCO}_{3}$ and the<smiles>O=C1CC=C(F)C(c2ccccc2)N1</smiles>
aqueous layer extracted with diethylether. The organic layers were combined and dried over $\mathrm{Na}_{2} \mathrm{SO}_{4}$. After filtration and evaporation of the volatile materials under reduced pressure, the crude was purified by flash chromatography on silica gel (Pentane: $\mathrm{Et}_{2} \mathrm{O}$ ) to afford the desired PMBprotected lactam.

5-fluoro-1,6-dihydropyridin-2(3H)-one (10a):

The general grocedure for the PMB-deprotection of the lactams using PMB-protected bisolefin 2a (1 eq., $100 \mathrm{mg}, 425 \mu \mathrm{mol}$ ) at room temperature during 7 hours followed by purification by chromatography on silica gel (Pentane: $\mathrm{Et}_{2} \mathrm{O}=80: 20$ ) afforded the 5-fluoro-1,6-dihydropyridin-2(3H)-one (40 mg, 348 $\mu$ mol, yield $81 \%$ ) as a white solid.

${ }^{1} \mathrm{H}$ NMR (400.13 MHz, MeOD-d6): $\delta(\mathrm{ppm}) 5.32\left(\mathrm{~m}, 1 \mathrm{H}, \mathrm{H}_{2}\right), 3.99\left(\mathrm{dd},{ }^{2} J_{\mathrm{H}-\mathrm{H}}=1 \mathrm{~Hz},{ }^{3} J_{\mathrm{H}-\mathrm{F}}=5 \mathrm{~Hz}, 2 \mathrm{H}\right.$, $\left.\mathrm{H}_{5}\right), 2.97\left(\mathrm{~m}, 2 \mathrm{H}, \mathrm{H}_{3}\right) .{ }^{19} \mathbf{F}$ NMR (282.40 MHz, MeOD-d6): $\delta(\mathrm{ppm})-119.93\left(\mathrm{dt},{ }^{3} J_{\mathrm{H}-\mathrm{F}}=5 \mathrm{~Hz},{ }^{3} J_{\mathrm{H}-\mathrm{F}}=\right.$ 15Hz, 1F, $\left.\mathrm{F}_{1}\right) .{ }^{13} \mathrm{C}$ NMR (75.47 MHz, MeOD-d6): $\delta(\mathrm{ppm}) 174.0\left(\mathrm{~s}, \mathrm{C}_{4}\right), 156.6\left(\mathrm{~d},{ }^{1} J_{\mathrm{C}-\mathrm{F}}=250 \mathrm{~Hz}, \mathrm{C}_{1}\right)$, $101.1\left(\mathrm{~d},{ }^{2} J_{\mathrm{C}-\mathrm{F}}=16 \mathrm{~Hz}, \mathrm{C}_{2}\right), 44.9\left(\mathrm{~d},{ }^{2} J_{\mathrm{C}-\mathrm{F}}=40 \mathrm{~Hz}, \mathrm{C}_{5}\right), 32.2\left(\mathrm{~d},{ }^{3} J_{\mathrm{C}-\mathrm{F}}=9 \mathrm{~Hz}, \mathrm{C}_{3}\right)$. IR (neat) $v 3208,1732$, 1668, 1372, 1275, 1159, 1018, 818, $750 \mathrm{~cm}^{-1}$. HR-MS (ESI+): $\mathrm{m} / \mathrm{z}=138.0332[\mathrm{M}+\mathrm{H}]^{+}$, calcd. for $\mathrm{C}_{5} \mathrm{H}_{6} \mathrm{FNONa}$ : 138.0331. $R f=0.17\left(20 \% \mathrm{Et}_{2} \mathrm{O}\right.$ in pentane $)$.

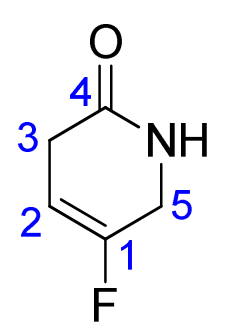

(S)-5-fluoro-6-phenyl-1,6-dihydropyridin-2(3H)-one (10d):

The general grocedure for the PMB-deprotection of the lactams using PMB-protected bisolefin $2 \mathbf{d}$ ( 1 eq., $30 \mathrm{mg}, 96 \mu \mathrm{mol}$ ) at room temperature during 14 hours followed by purification by chromatography on silica gel (Pentane: $\left.\mathrm{Et}_{2} \mathrm{O}=80: 20\right)$ afforded the $(\boldsymbol{S})$-5- 
fluoro-6-phenyl-1,6-dihydropyridin-2(3H)-one (14 mg, $73 \mu \mathrm{mol}$, yield $76 \%)$ as a white solid.

${ }^{1} \mathbf{H}$ NMR (400.13 MHz, $\left.\mathbf{C D C l}_{3}\right)$ : $\delta$ (ppm) 7.39 (m, 5H, $\mathrm{H}_{7,8,9),} 6.00$ (br., $\left.1 \mathrm{H}, \mathrm{NH}\right), 5.36\left(\mathrm{ddt},{ }^{3} J_{\mathrm{H}-\mathrm{H}}=\right.$ $\left.1 \mathrm{~Hz},{ }^{3} J_{\mathrm{H}-\mathrm{H}}=4 \mathrm{~Hz},{ }^{3} J_{\mathrm{H}-\mathrm{F}}=14 \mathrm{~Hz}, 1 \mathrm{H}, \mathrm{H}_{2}\right), 5.10\left(\mathrm{~m}, 1 \mathrm{H}, \mathrm{H}_{5}\right), 3.15\left(\mathrm{~m}, 2 \mathrm{H}, \mathrm{H}_{3}\right) .{ }^{19} \mathbf{F}$ NMR $(282.40 \mathrm{MHz}$, $\left.\mathbf{C D C l}_{3}\right): \delta(\mathrm{ppm})-117.69\left(\mathrm{~m}, 1 \mathrm{~F}, \mathrm{~F}_{1}\right) .{ }^{13} \mathbf{C} \mathbf{N M R}\left(\mathbf{7 5 . 4 7} \mathbf{M H z}, \mathbf{C D C l}_{3}\right): \delta(\mathrm{ppm}) 167.9\left(\mathrm{~s}, \mathrm{C}_{4}\right), 153.9(\mathrm{~d}$, $\left.{ }^{1} J_{\mathrm{C}-\mathrm{F}}=251 \mathrm{~Hz}, \mathrm{C}_{1}\right), 138.0\left(\mathrm{~s}, 1 \mathrm{C}, \mathrm{C}_{7}\right), 129.1\left(\mathrm{~s}, 2 \mathrm{C}, \mathrm{C}_{7}\right), 127.2\left(\mathrm{~s}, 2 \mathrm{C}, \mathrm{C}_{8}\right), 98.0\left(\mathrm{~d},{ }^{2} J_{\mathrm{C}-\mathrm{F}}=17 \mathrm{~Hz}, \mathrm{C}_{2}\right), 57.4$ $\left(\mathrm{d},{ }^{2} J_{\mathrm{C}-\mathrm{F}}=37 \mathrm{~Hz}, \mathrm{C}_{5}\right), 29.1\left(\mathrm{~d},{ }^{3} J_{\mathrm{C}-\mathrm{F}}=8 \mathrm{~Hz}, \mathrm{C}_{3}\right)$. IR (neat) $v 1754,1663,1627,1530,1491,1476,1399$, 1376, 1313, 1274, 1193, 1163, 1119, 1021, 838, 742, $697 \mathrm{~cm}^{-1}$. HR-MS (ESI+): $\mathrm{m} / \mathrm{z}=192.0818$ $[\mathrm{M}+\mathrm{H}]^{+}$, calcd. for $\mathrm{C}_{11} \mathrm{H}_{11} \mathrm{FNO}: 192.0825 .[\boldsymbol{\alpha}]_{\mathbf{D}}^{25}=+112.7$ (c 1.1, CH3Cl). Chiral HPLC: Method conditions: Col. Daicel Chiralpak ASH $4.6 \mathrm{mmx} 250 \mathrm{~mm} 5 \mu \mathrm{m}, 1 \mathrm{~mL} / \mathrm{min}, 20^{\circ} \mathrm{C}, 80 \%$ n-heptane $20 \%$ propanol; peak 1: $\operatorname{tr}=9.300 \mathrm{~min}, \%$ Area $=99.10 \%$; peak $2: \operatorname{tr}=11.104 \mathrm{~min}, \%$ Area $=0.90 \% \cdot R f=0.15$ $\left(20 \% \mathrm{Et}_{2} \mathrm{O}\right.$ in pentane).

\section{5-fluoro-6-phenyl-1,6-dihydropyridin-2(3H)-one (rac-10d):}

The general grocedure for the PMB-deprotection of the lactams using PMB-protected bisolefin $\mathbf{2 d}+\mathbf{e}$ (1 eq., $21 \mathrm{mg}, 68 \mu \mathrm{mol})$ at room temperature during 14 hours followed by purification by chromatography on silica gel (Pentane: $\mathrm{Et}_{2} \mathrm{O}=80: 20$ ) afforded the 5-fluoro-6-phenyl-1,6-dihydropyridin-2(3H)-one (7 $\mathrm{mg}, 37 \mu \mathrm{mol}$, yield $55 \%$ ) as a white solid.

${ }^{1} \mathbf{H}$ NMR (400.13 MHz, CDCl $)$ : $\delta$ (ppm) $7.38\left(\mathrm{~m}, 5 \mathrm{H}, \mathrm{H}_{7,8,9}\right), 6.00$ (br., $\left.1 \mathrm{H}, \mathrm{NH}\right), 5.36$ (ddt, ${ }^{3} J_{\mathrm{H}-\mathrm{H}}=$ $\left.1 \mathrm{~Hz},{ }^{3} J_{\mathrm{H}-\mathrm{H}}=4 \mathrm{~Hz},{ }^{3} J_{\mathrm{H}-\mathrm{F}}=14 \mathrm{~Hz}, 1 \mathrm{H}, \mathrm{H}_{2}\right), 5.10\left(\mathrm{~m}, 1 \mathrm{H}, \mathrm{H}_{5}\right), 3.16\left(\mathrm{~m}, 2 \mathrm{H}, \mathrm{H}_{3}\right) .{ }^{19} \mathbf{F}$ NMR $(282.40 \mathrm{MHz}$, $\left.\mathbf{C D C l}_{3}\right): \delta(\mathrm{ppm})-117.68\left(\mathrm{~m}, 1 \mathrm{~F}, \mathrm{~F}_{1}\right)$. Racemic mixture: Chiral HPLC: Method conditions: Col. Daicel Chiralpak ASH $4.6 \mathrm{mmx} 250 \mathrm{~mm} 5 \mu \mathrm{m}, 1 \mathrm{~mL} / \mathrm{min}, 20^{\circ} \mathrm{C}, 80 \%$ n-heptane $20 \%$ propanol; peak $1: \operatorname{tr}=9.283$ $\min , \%$ Area $=50.36 \%$; peak $2: \operatorname{tr}=11.070 \mathrm{~min}, \%$ Area $=49.64 \%$.

\section{b. Synthesis of the fluoropseudopeptides 1}

\section{General procedure for the hydrolysis of lactams:}<smiles>O=C1CC=C(F)C(c2ccccc2)N1</smiles>

In an oven dried Schlenk flask equipped with a stir bar and flushed with argon, a solution of the starting lactam (1 eq.), in $\mathrm{HCl} 6 \mathrm{~N}$ (30 eq.) was prepared. The resulting solution was stirred at $100^{\circ} \mathrm{C}$ and the progress of the reaction was controlled by TLC and ${ }^{19} \mathrm{~F}$ NMR. When full consumption of starting material was observed, the vial content was evaporated under reduced pressure. The crude 
was washed with dichloromethane to afford the pure desired fluoropseudopeptide.

(E)-5-amino-4-fluoropent-3-enoic acid chlorhydrate (1a):

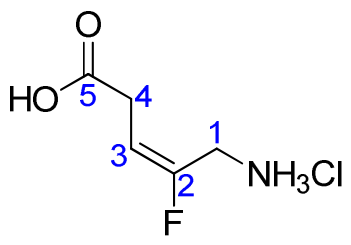

The general grocedure for the hydrolysis of the lactams using PMBdeprotected lactam 10a (1 eq., $8 \mathrm{mg}, 70 \mu \mathrm{mol})$ afforded the $(\boldsymbol{E})-5-$ amino-4-fluoropent-3-enoic acid chlorhydrate quantitatively as a white solid.

${ }^{1} \mathbf{H}$ NMR (400.13 MHz, D 20$): \delta(\mathrm{ppm}) 5.62\left(\mathrm{dt},{ }^{3} J_{\mathrm{H}-\mathrm{H}}=8 \mathrm{~Hz},{ }^{3} J_{\mathrm{H}-\mathrm{F}}=19 \mathrm{~Hz}, 1 \mathrm{H}, \mathrm{H}_{3}\right), 3.84\left(\mathrm{~d},{ }^{3} J_{\mathrm{H}-\mathrm{F}}=\right.$ $\left.19 \mathrm{~Hz}, 2 \mathrm{H}, \mathrm{H}_{1}\right), 3.15\left(\mathrm{~d},{ }^{3} J_{\mathrm{H}-\mathrm{H}}=8 \mathrm{~Hz}, 2 \mathrm{H}, \mathrm{H}_{4}\right) .{ }^{19} \mathbf{F}$ NMR (282.40 MHz, $\left.\mathbf{D}_{2} \mathbf{O}\right): \delta(\mathrm{ppm})-110.11\left(\mathrm{dt},{ }^{3} J_{\mathrm{H}-\mathrm{F}}\right.$ $\left.=19 \mathrm{~Hz},{ }^{3} J_{\mathrm{H}-\mathrm{F}}=19 \mathrm{~Hz}, 1 \mathrm{~F}, \mathrm{~F}_{2}\right) .{ }^{13} \mathbf{C}$ NMR $\left(75.47 \mathbf{~ M H z}, \mathbf{D}_{2} \mathbf{O}\right): \delta(\mathrm{ppm}) 175.1\left(\mathrm{~d},{ }^{4} J_{\mathrm{C}-\mathrm{F}}=3 \mathrm{~Hz}, \mathrm{C}_{5}\right), 154.5$ $\left(\mathrm{d},{ }^{1} J_{\mathrm{C}-\mathrm{F}}=247 \mathrm{~Hz}, \mathrm{C}_{2}\right), 105.9\left(\mathrm{~d},{ }^{2} J_{\mathrm{C}-\mathrm{F}}=25 \mathrm{~Hz}, \mathrm{C}_{3}\right), 35.8\left(\mathrm{~d},{ }^{2} J_{\mathrm{C}-\mathrm{F}}=29 \mathrm{~Hz}, \mathrm{C}_{1}\right), 30.2\left(\mathrm{~d},{ }^{3} J_{\mathrm{C}-\mathrm{F}}=10 \mathrm{~Hz}, \mathrm{C}_{4}\right)$. IR (neat) $v 3370,3011,2620,2510,1713,1634,1506,1396,1306,1206,1162,1076,989,937,851$ $\mathrm{cm}^{-1}$. HR-MS (ESI+): $\mathrm{m} / \mathrm{z}=134.0612[\mathrm{M}+\mathrm{H}]^{+}$, calcd. for $\mathrm{C}_{5} \mathrm{H}_{9} \mathrm{FNO}_{2}: 134.0617$.

(S)-(E)-5-amino-4-fluoro-5-phenylpent-3-enoic acid chlorhydrate (1d):<smiles>N[C@@H](F)/C(F)=C\C(=O)O</smiles>

The general grocedure for the hydrolysis of the lactams using PMBdeprotected lactam 10d (1 eq., $8 \mathrm{mg}, 42 \mu \mathrm{mol}$ ) afforded the (S)-(E)5-amino-4-fluoro-5-phenylpent-3-enoic acid chlorhydrate quantitatively as a white solid.

${ }^{1} \mathbf{H}$ NMR (400.13 MHz, D2 2 ): $\delta(\mathrm{ppm}) 7.45\left(\mathrm{~s}, 5 \mathrm{H}, \mathrm{H}_{7,8,9)}, 5.65\left(\mathrm{dt},{ }^{3} J_{\mathrm{H}-\mathrm{H}}=8 \mathrm{~Hz},{ }^{3} J_{\mathrm{H}-\mathrm{F}}=21 \mathrm{~Hz}, 1 \mathrm{H}, \mathrm{H}_{3}\right)\right.$, $5.49\left(\mathrm{~d},{ }^{3} \boldsymbol{J}_{\mathrm{H}-\mathrm{F}}=25 \mathrm{~Hz}, 1 \mathrm{H}, \mathrm{H}_{1}\right), 3.17\left(\mathrm{~m}, 2 \mathrm{H}, \mathrm{H}_{4}\right) .{ }^{19} \mathbf{F}$ NMR $\left(282.40 \mathbf{M H z}, \mathbf{D}_{2} \mathbf{O}\right): \delta(\mathrm{ppm})-117.2(\mathrm{dd}$, $\left.{ }^{3} J_{\mathrm{H}-\mathrm{F}}=21 \mathrm{~Hz},{ }^{3} J_{\mathrm{H}-\mathrm{F}}=25 \mathrm{~Hz}, 1 \mathrm{~F}, \mathrm{~F}_{2}\right) .{ }^{13} \mathbf{C} \mathbf{N M R}\left(75.47 \mathbf{~ M H z}, \mathbf{D}_{2} \mathrm{O}\right): \delta(\mathrm{ppm}) 175.0\left(\mathrm{~d},{ }^{4} J_{\mathrm{C}-\mathrm{F}}=4 \mathrm{~Hz}, \mathrm{C}_{5}\right)$, $154.6\left(\mathrm{~d},{ }^{1} J_{\mathrm{C}-\mathrm{F}}=253 \mathrm{~Hz}, \mathrm{C}_{2}\right), 132.5\left(\mathrm{~d},{ }^{3} J_{\mathrm{C}-\mathrm{F}}=2 \mathrm{~Hz}, \mathrm{C}_{6}\right), 129.8\left(\mathrm{~s}, \mathrm{C}_{9}\right), 129.5\left(\mathrm{~s}, 2 \mathrm{C}, \mathrm{C}_{8}\right), 127.0\left(\mathrm{~d},{ }^{4} J_{\mathrm{C}-\mathrm{F}}=\right.$ $\left.2 \mathrm{~Hz}, 2 \mathrm{C}, \mathrm{C}_{7}\right), 105.1\left(\mathrm{~d},{ }^{2} J_{\mathrm{C}-\mathrm{F}}=24 \mathrm{~Hz}, \mathrm{C}_{3}\right), 51.0\left(\mathrm{~d},{ }^{2} J_{\mathrm{C}-\mathrm{F}}=25 \mathrm{~Hz}, \mathrm{C}_{1}\right), 30.1\left(\mathrm{~d},{ }^{3} J_{\mathrm{C}-\mathrm{F}}=10 \mathrm{~Hz}, \mathrm{C}_{4}\right) . \mathbf{I R}($ neat $)$ $v$ 3395, 3136, 2865, 1721, 1638, 1594, 1567, 1515, 1495, 1400, 1377, 1297, 1191, 1185, 1160, 1110, 1093, 991, 934, 906, 836, 753, $696 \mathrm{~cm}^{-1}$. HR-MS (ESI+): $\mathrm{m} / \mathrm{z}=210.0937[\mathrm{M}+\mathrm{H}]^{+}$, calcd. for $\mathrm{C}_{11} \mathrm{H}_{13} \mathrm{FNO}_{2}:$ 210.0930. $[\boldsymbol{\alpha}]_{\mathbf{D}}^{25}=-107\left(c 0.75, \mathrm{D}_{2} \mathrm{O}\right)$. 
<smiles>Nc1ccc(CC(=O)O)cc1F</smiles>

The general grocedure for the hydrolysis of the lactams using PMBdeprotected lactam rac-10d (1 eq., $8 \mathrm{mg}, 42 \mu \mathrm{mol}$ ) afforded the $(\boldsymbol{E})$ 5-amino-4-fluoro-5-phenylpent-3-enoic acid chlorhydrate quantitatively as a white solid.

${ }^{1} \mathbf{H}$ NMR (400.13 MHz, D2 $\left.\mathbf{O}\right): \delta(\mathrm{ppm}) 7.45\left(\mathrm{~s}, 5 \mathrm{H}, \mathrm{H}_{7,8,9)}, 5.65\left(\mathrm{dt},{ }^{3} J_{\mathrm{H}-\mathrm{H}}=8 \mathrm{~Hz},{ }^{3} J_{\mathrm{H}-\mathrm{F}}=21 \mathrm{~Hz}, 1 \mathrm{H}, \mathrm{H}_{3}\right)\right.$, $5.49\left(\mathrm{~d},{ }^{3} \boldsymbol{J}_{\mathrm{H}-\mathrm{F}}=25 \mathrm{~Hz}, 1 \mathrm{H}, \mathrm{H}_{1}\right), 3.05\left(\mathrm{~m}, 2 \mathrm{H}, \mathrm{H}_{4}\right) .{ }^{19} \mathbf{F}$ NMR $\left(\mathbf{2 8 2 . 4 0} \mathbf{M H z}, \mathbf{D}_{2} \mathbf{O}\right): \delta(\mathrm{ppm})-117.2(\mathrm{dd}$, $\left.{ }^{3} J_{\mathrm{H}-\mathrm{F}}=21 \mathrm{~Hz},{ }^{3} J_{\mathrm{H}-\mathrm{F}}=25 \mathrm{~Hz}, 1 \mathrm{~F}, \mathrm{~F}_{2}\right)$. 
V. ${ }^{1} \mathrm{H},{ }^{19} \mathrm{~F}$ and ${ }^{13} \mathrm{C}$ Spectra for compounds 1a,d, 2b-e,h, 3a-h, 4a-c, 5, 7 a-h, 9c,e,g, 10a,d

Compound 1a: (E)-5-amino-4-fluoropent-3-enoic acid chlorhydrate
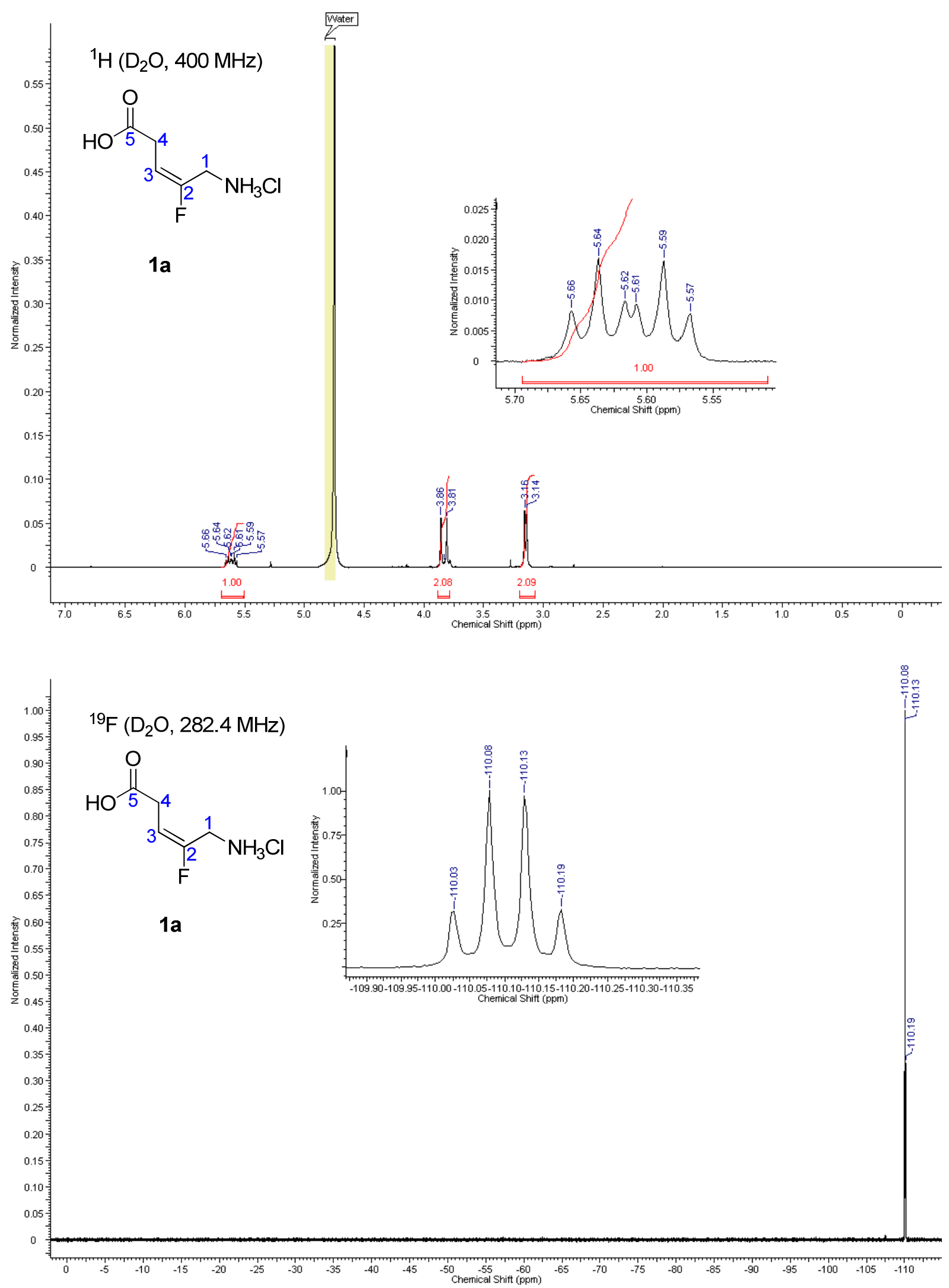


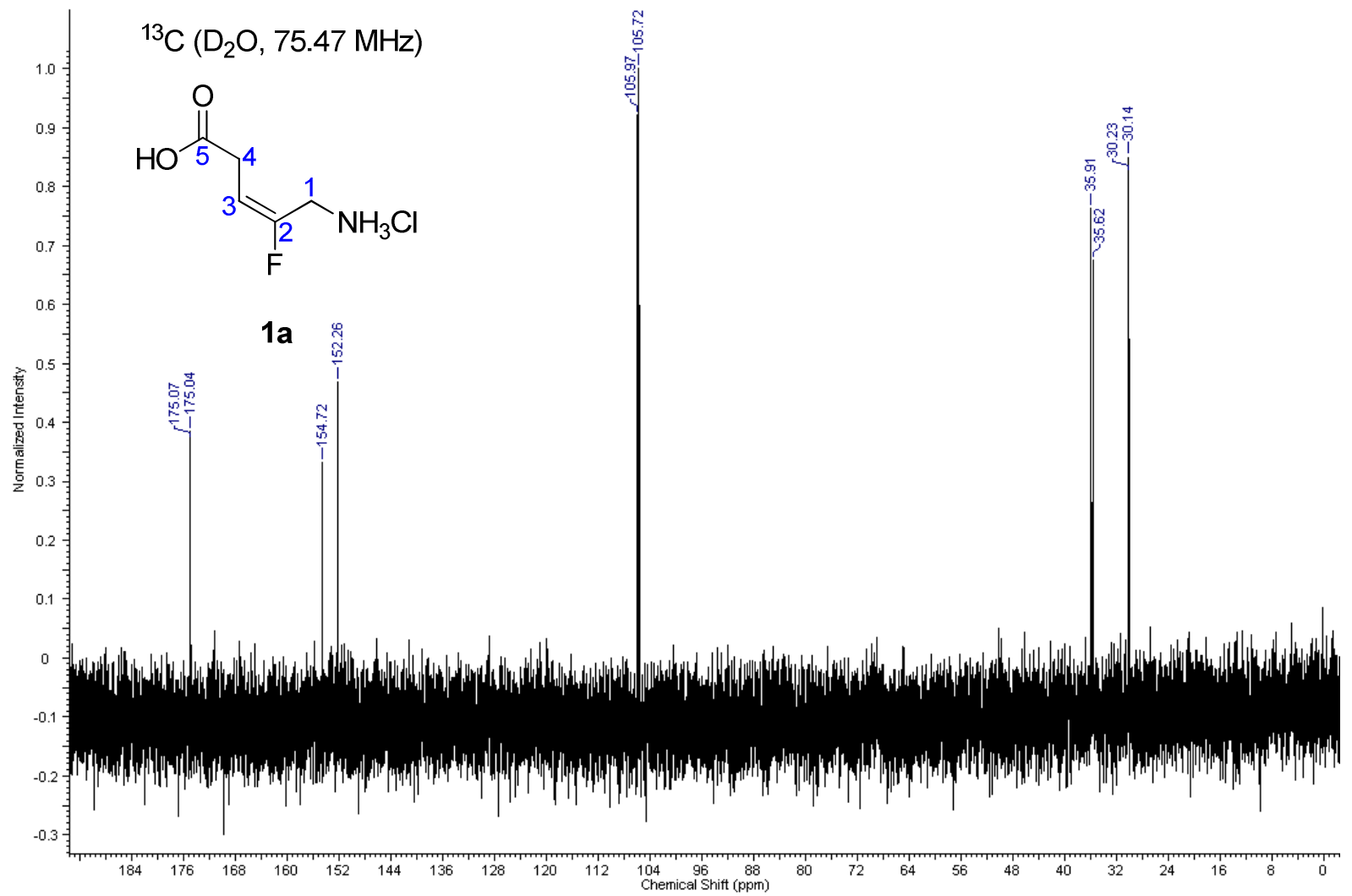

Compound 1d: $(S)-(E)$-5-amino-4-fluoro-5-phenylpent-3-enoic acid chlorhydrate (1d)

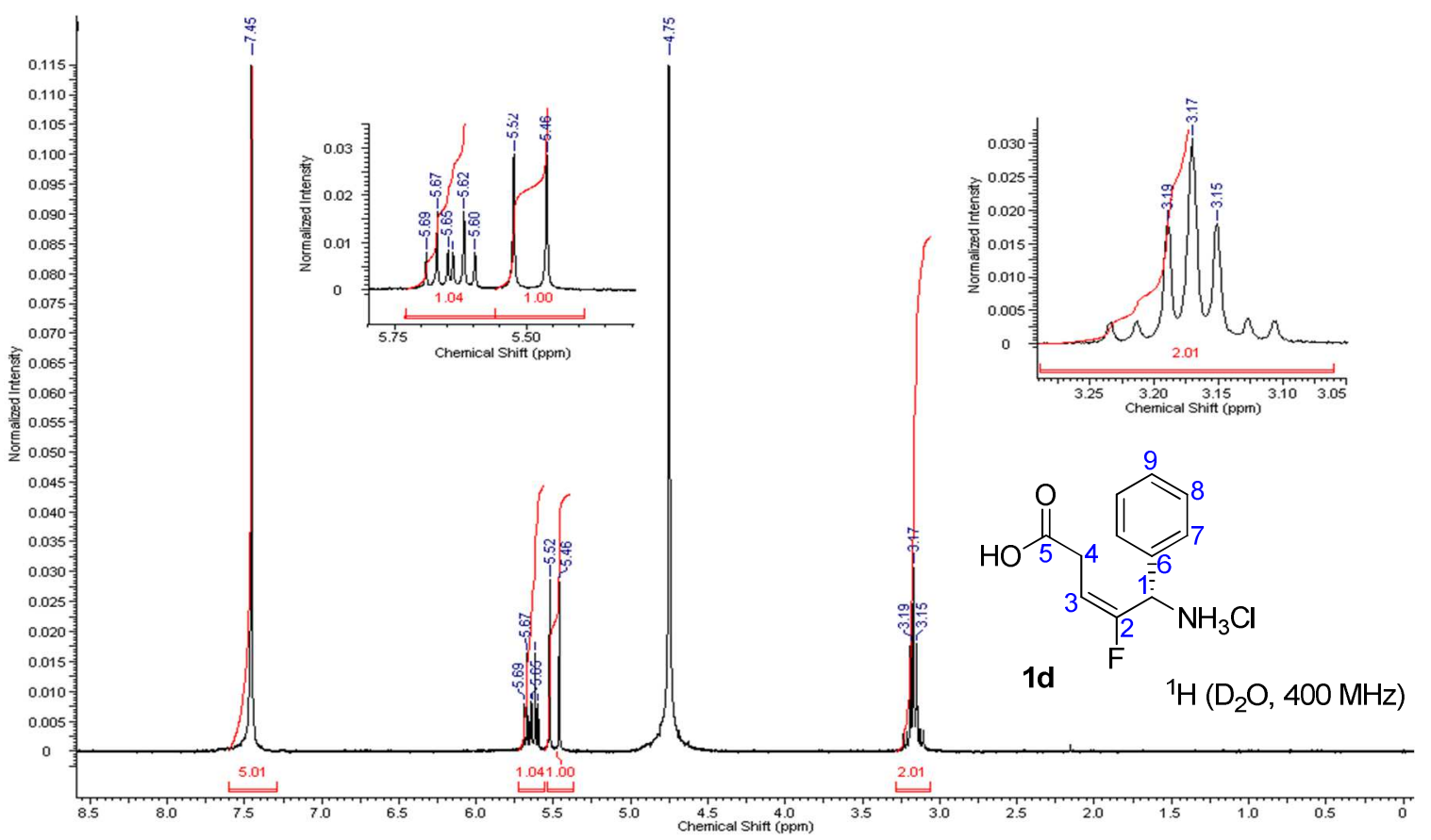



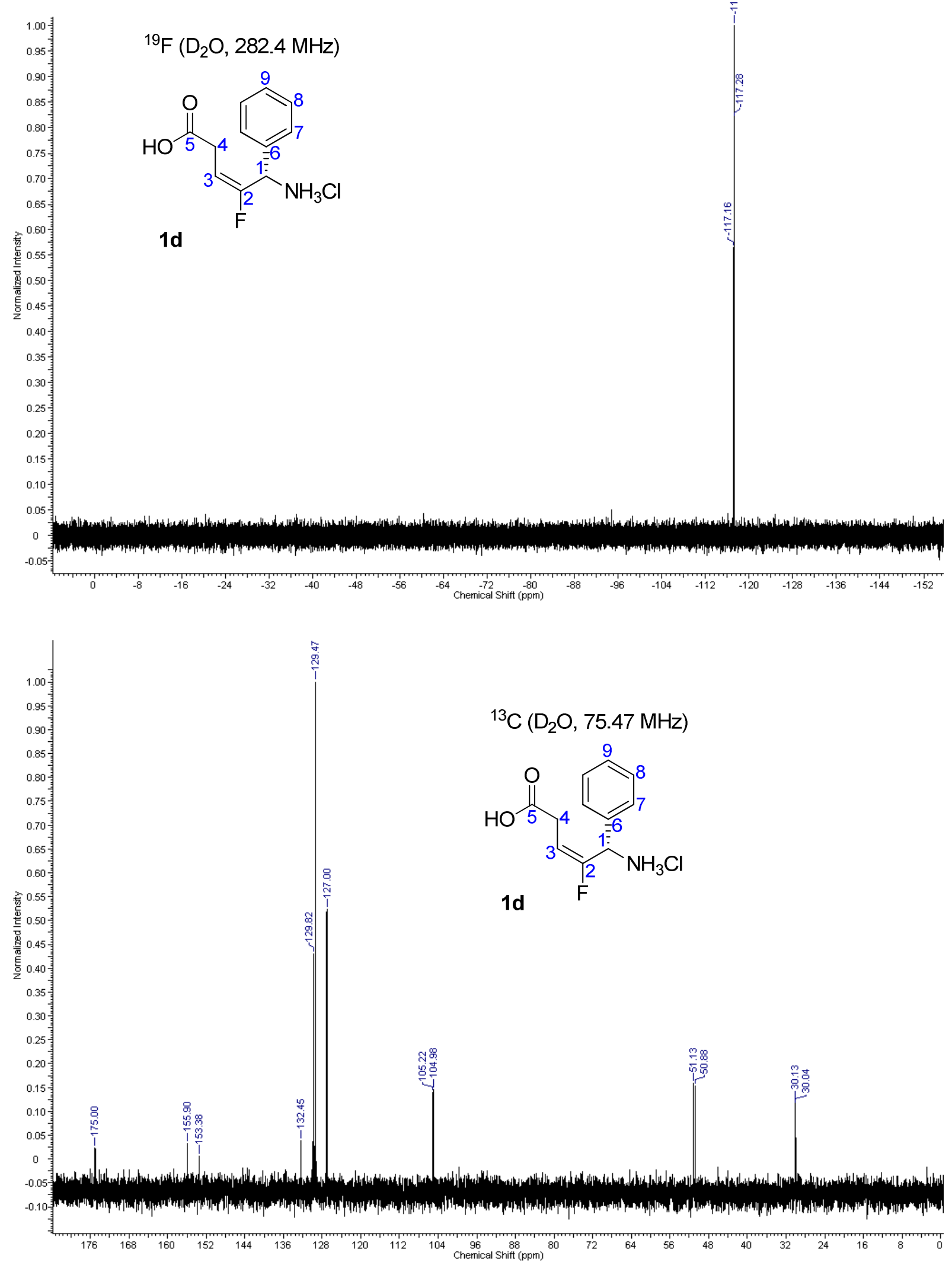
Compound rac-1d: (E)-5-amino-4-fluoro-5-phenylpent-3-enoic acid chlorhydrate
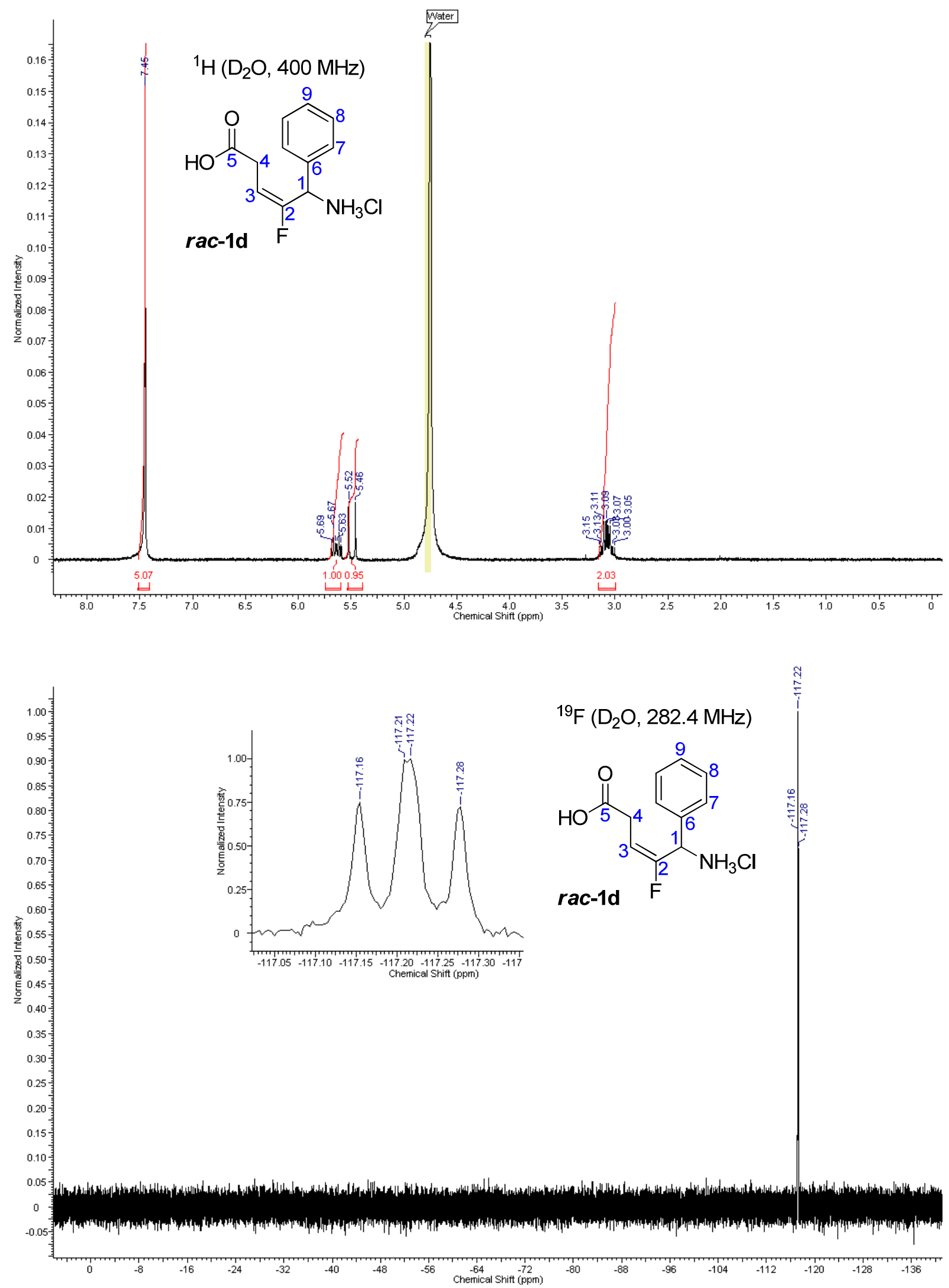
Compound 2b: (S)-5-fluoro-1-(4-methoxybenzyl)-6-methyl-1,6-dihydropyridin-2(3H)-one
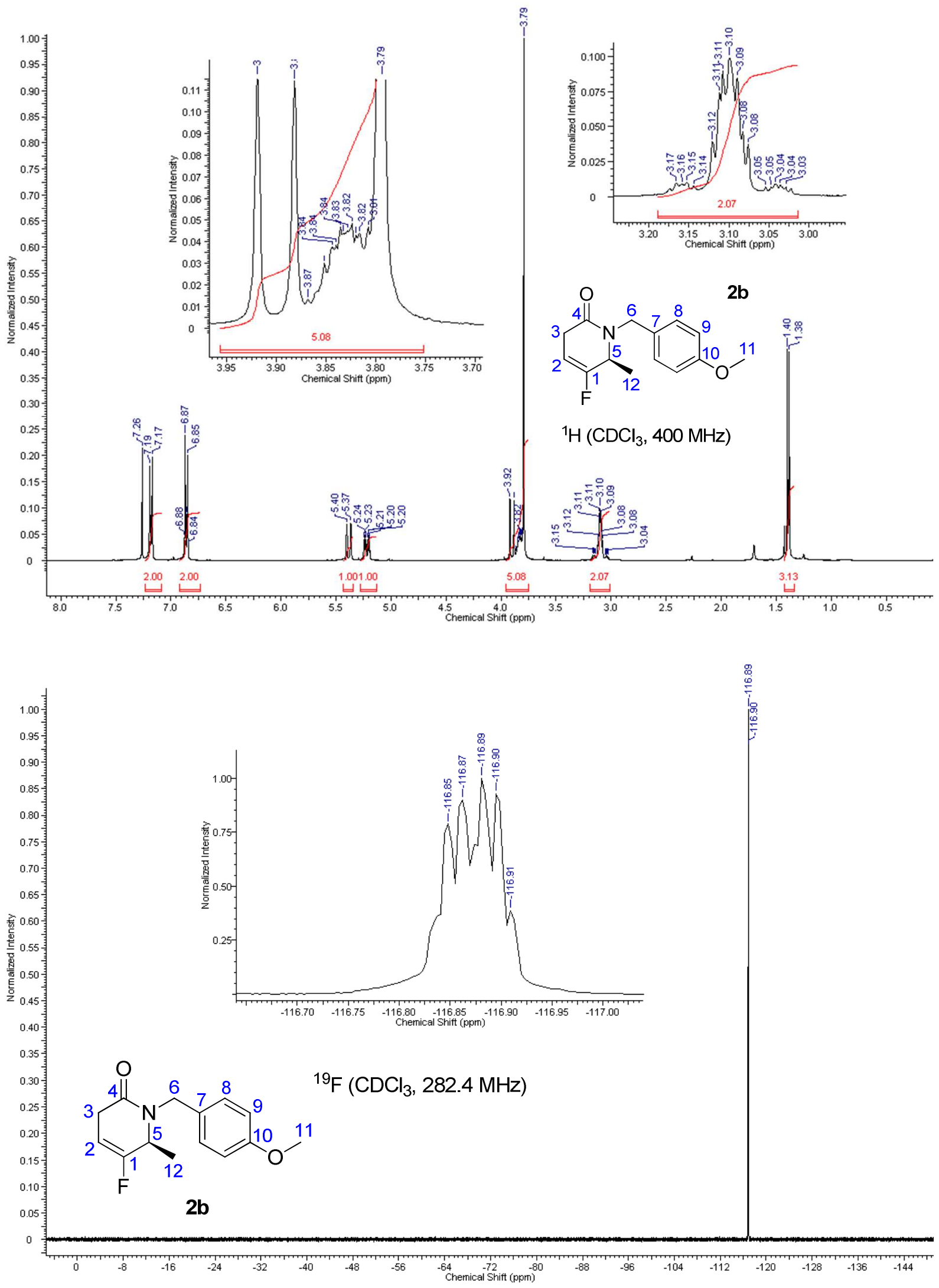


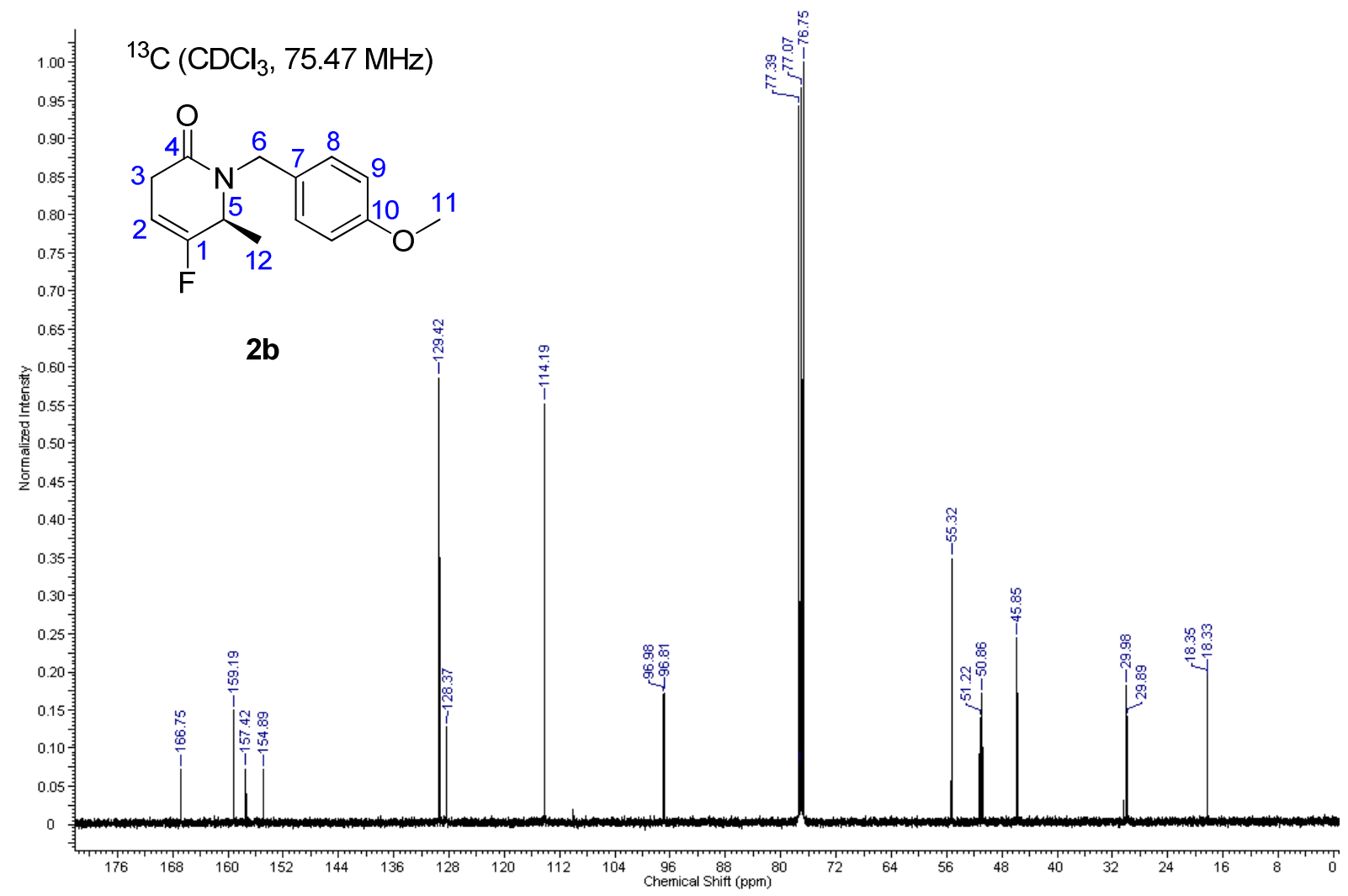

Compound 2c: (R)-5-fluoro-1-(4-methoxybenzyl)-6-methyl-1,6-dihydropyridin-2(3H)-one

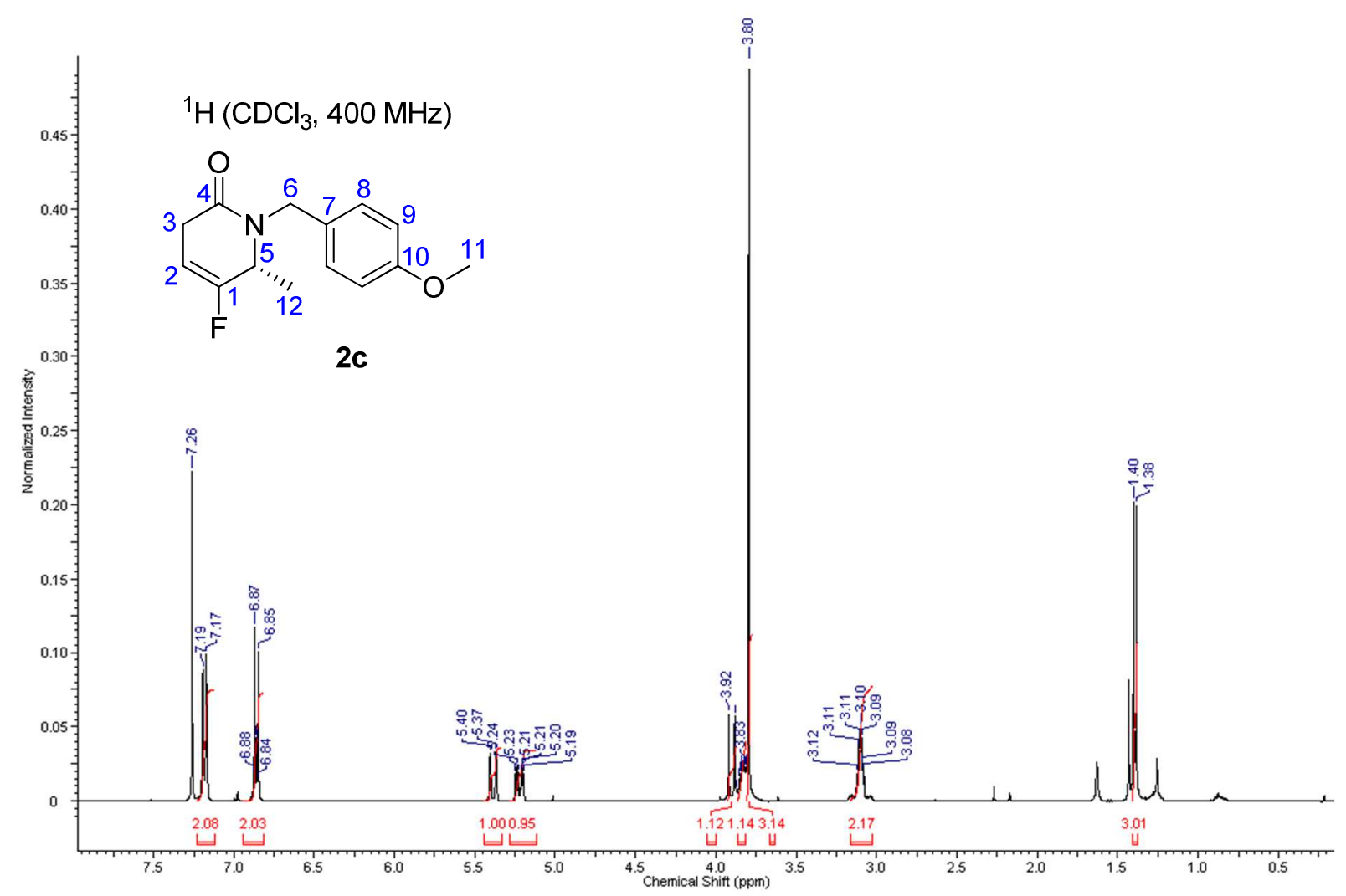



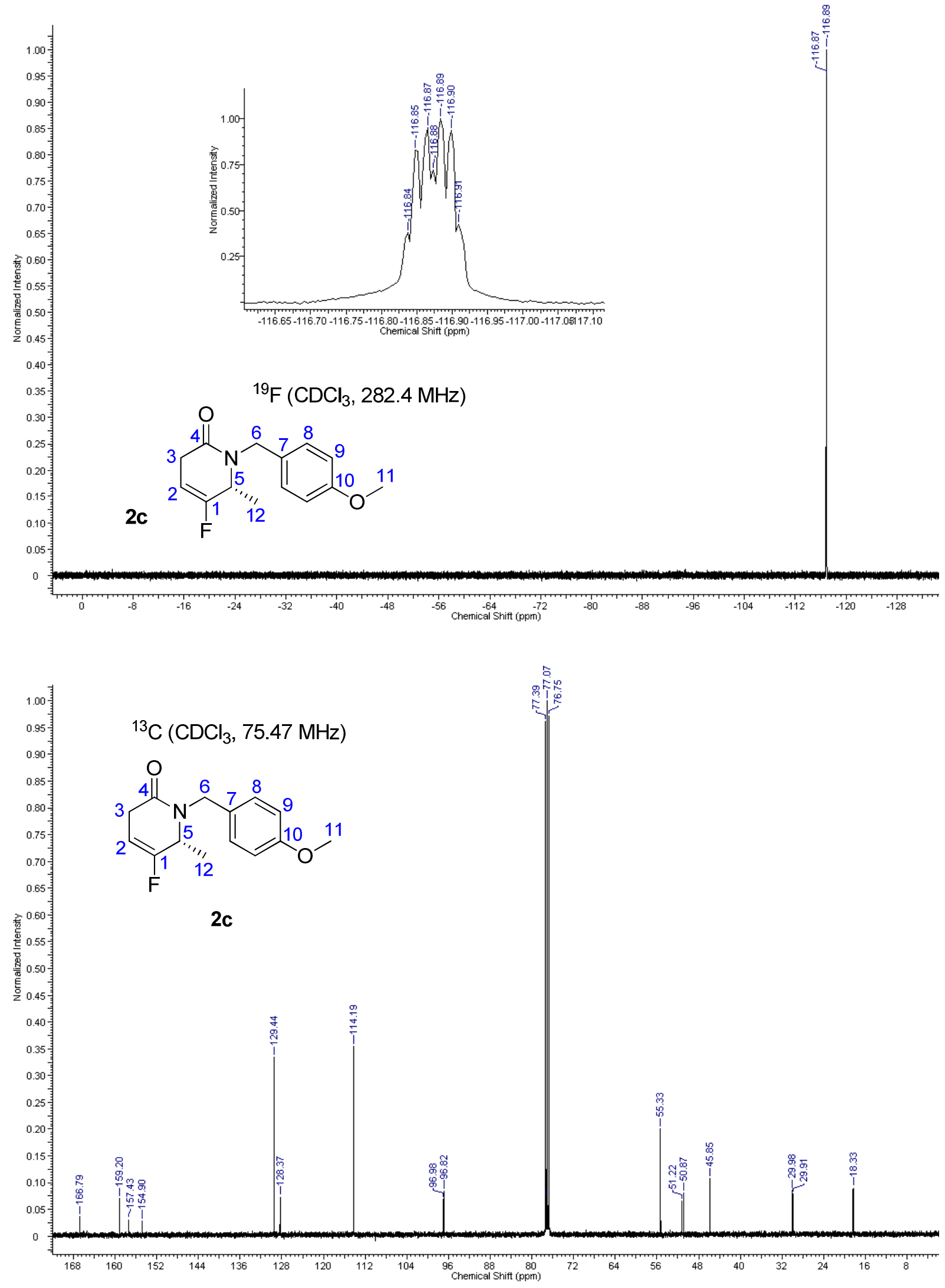
Compound 2d: (S)-5-fluoro-1-(4-methoxybenzyl)-6-phenyl-1,6-dihydropyridin-2(3H)-one
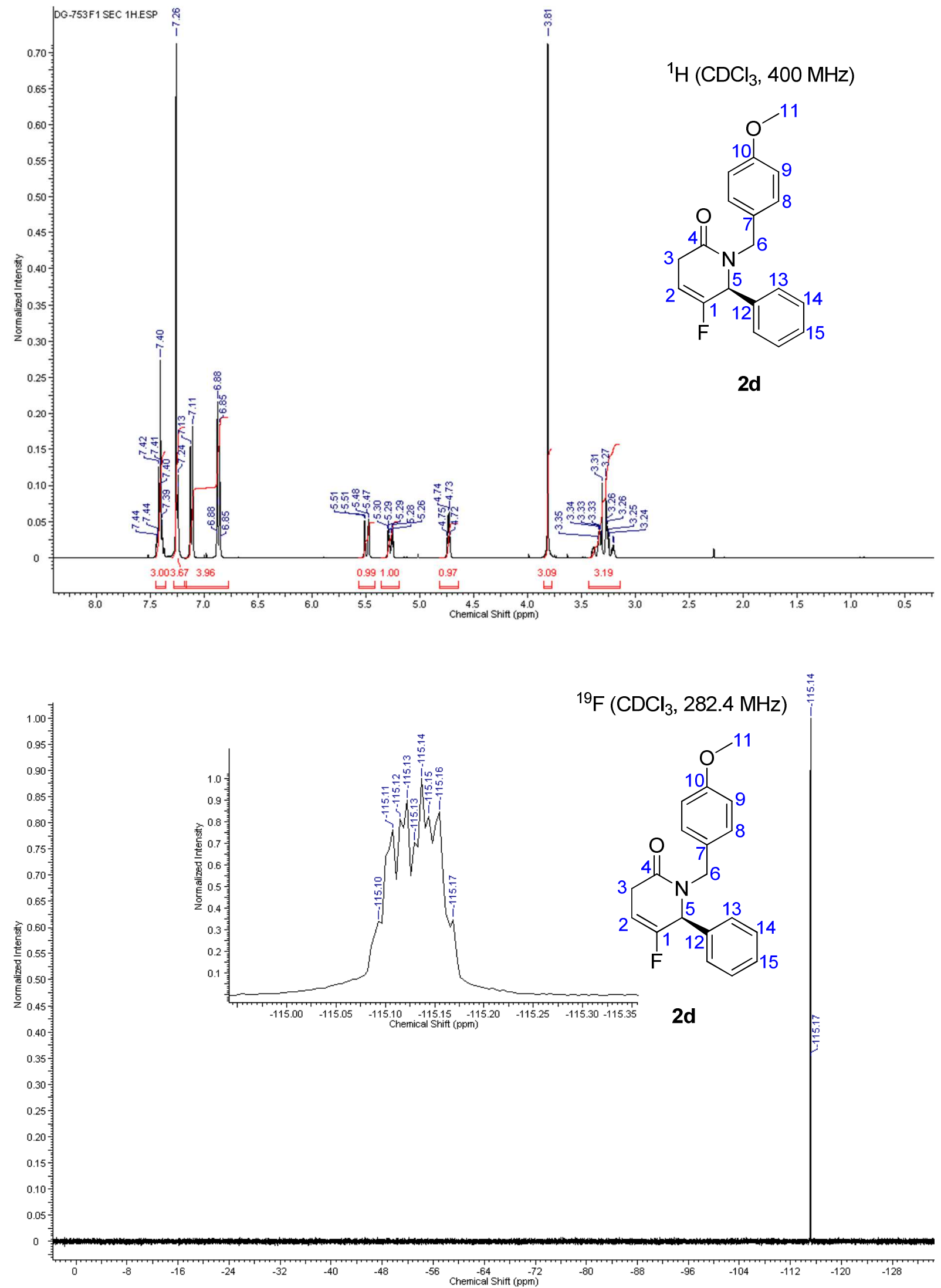


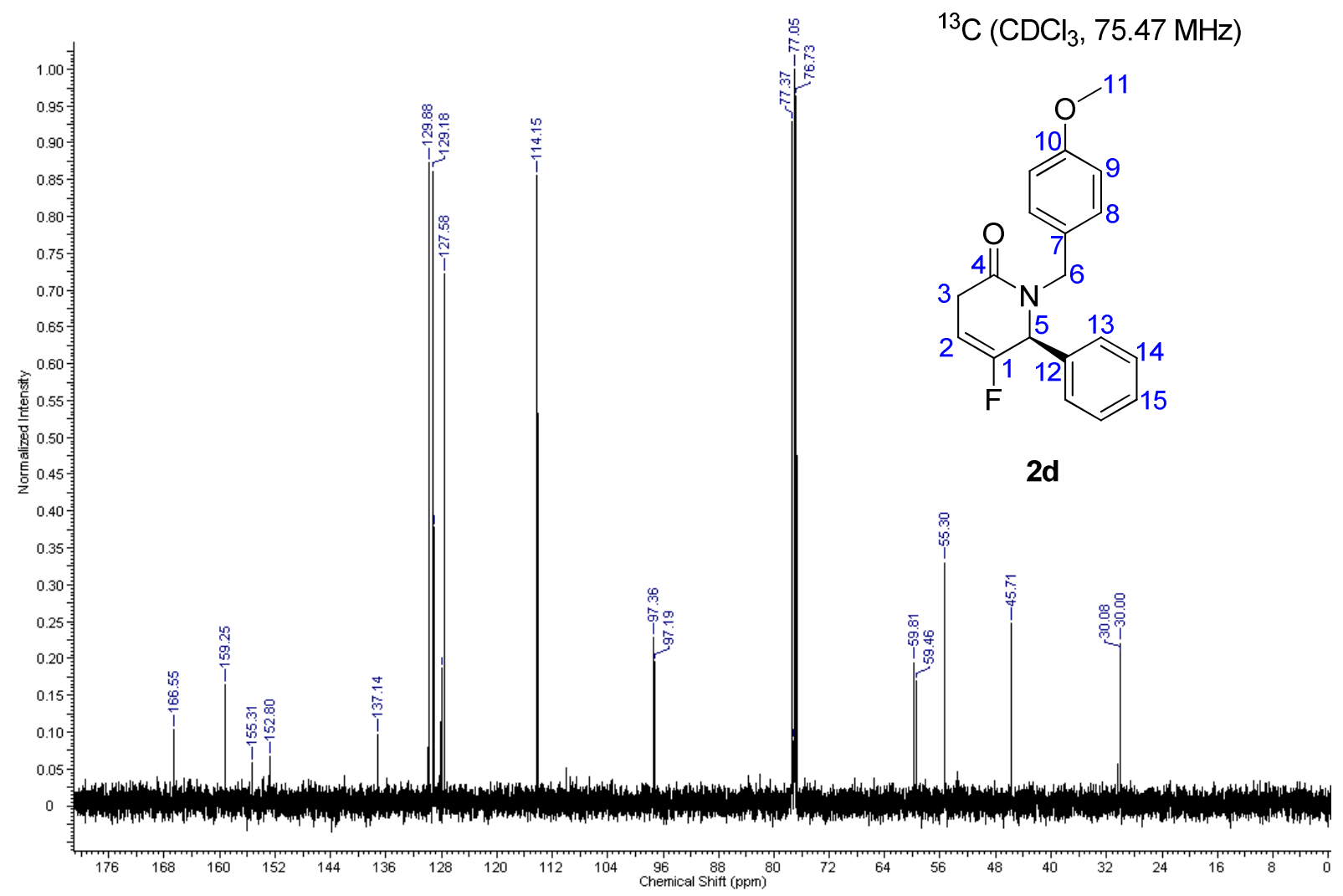

Compound 2d+e: Racemic 5-fluoro-1-(4-methoxybenzyl)-6-phenyl-1,6-dihydropyridin-2(3H)one

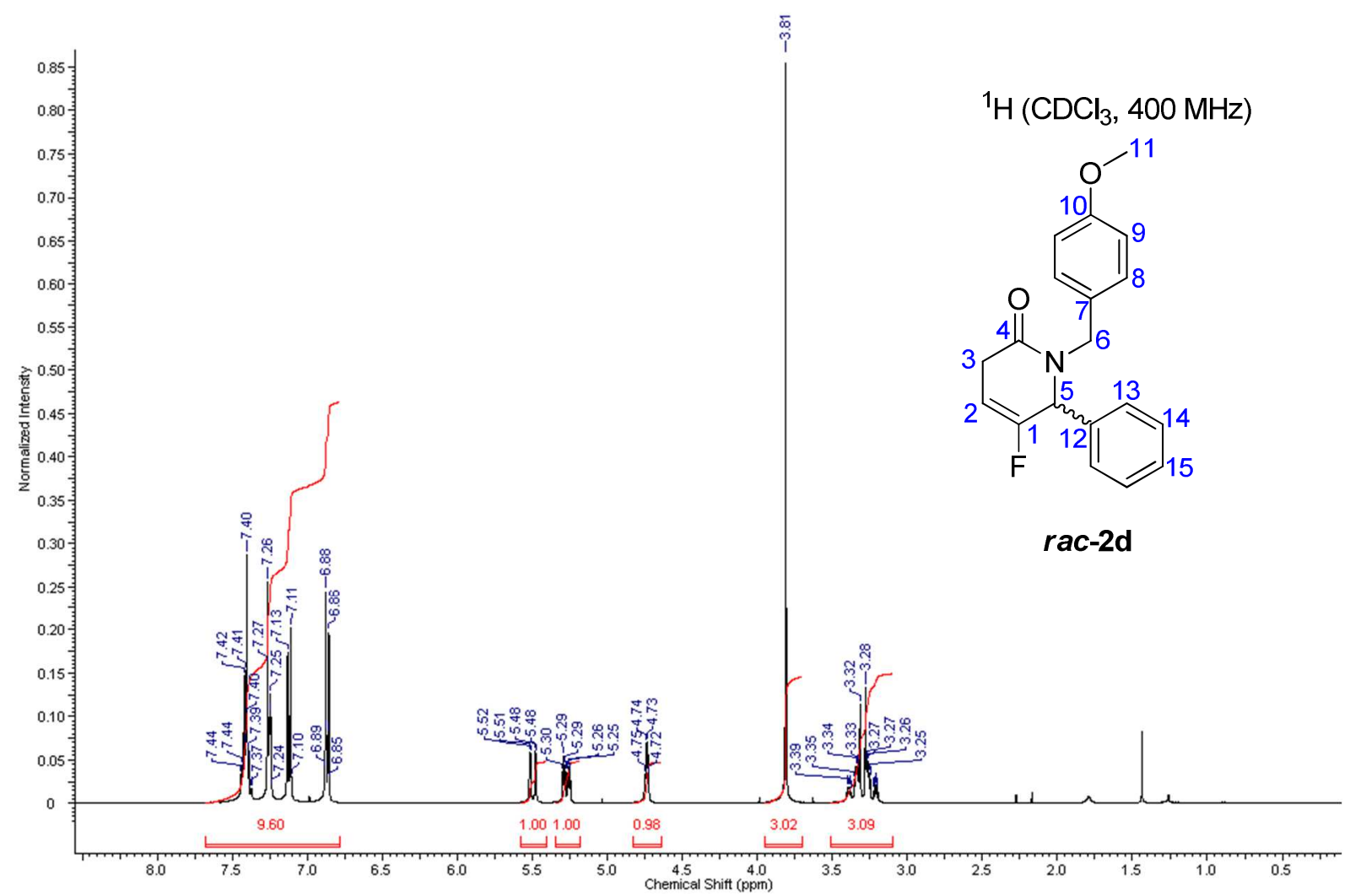



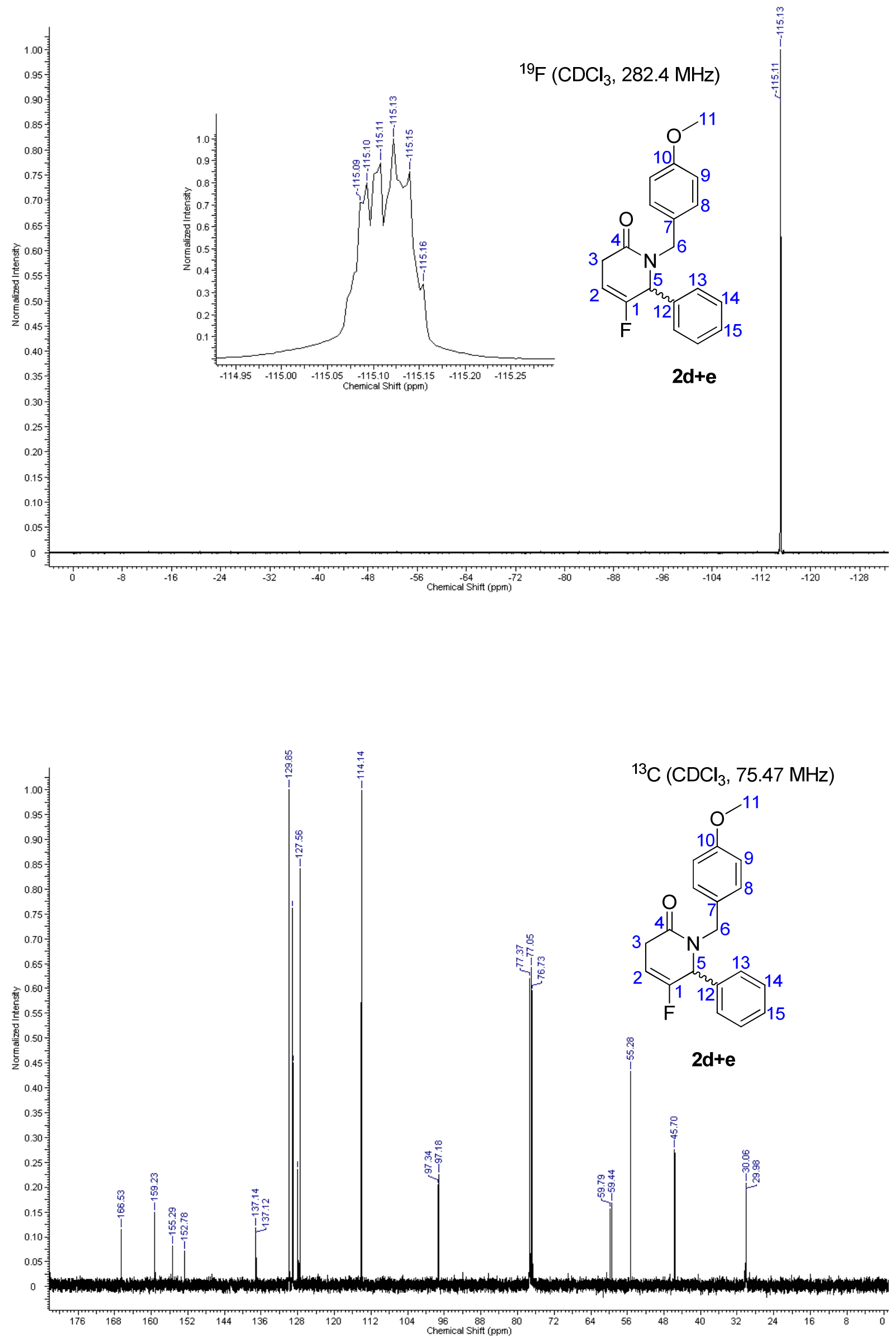
Compound 2h: 6-benzyl-5-fluoro-1-(4-methoxybenzyl)-1,6-dihydropyridin-2(3H)-one
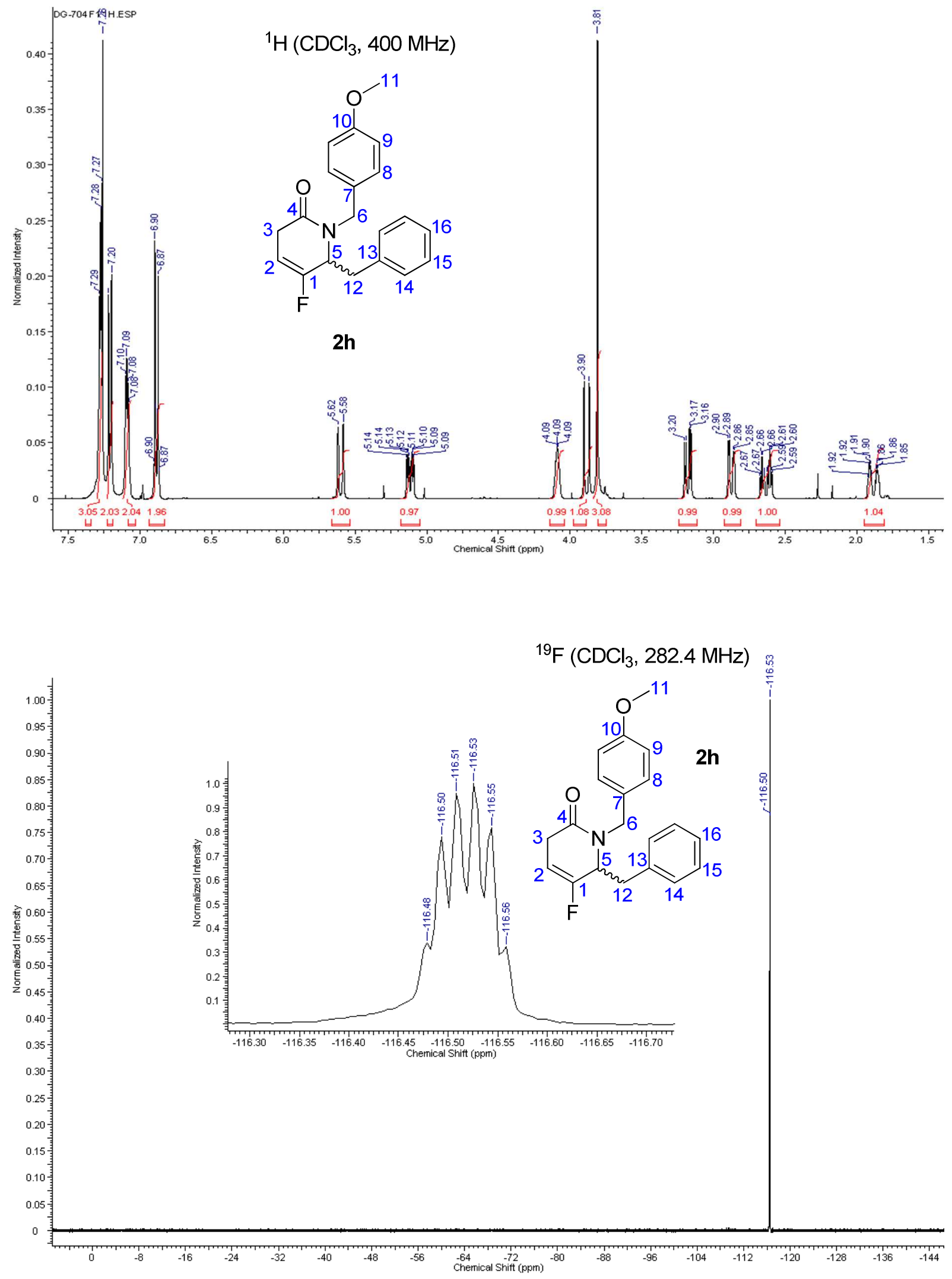


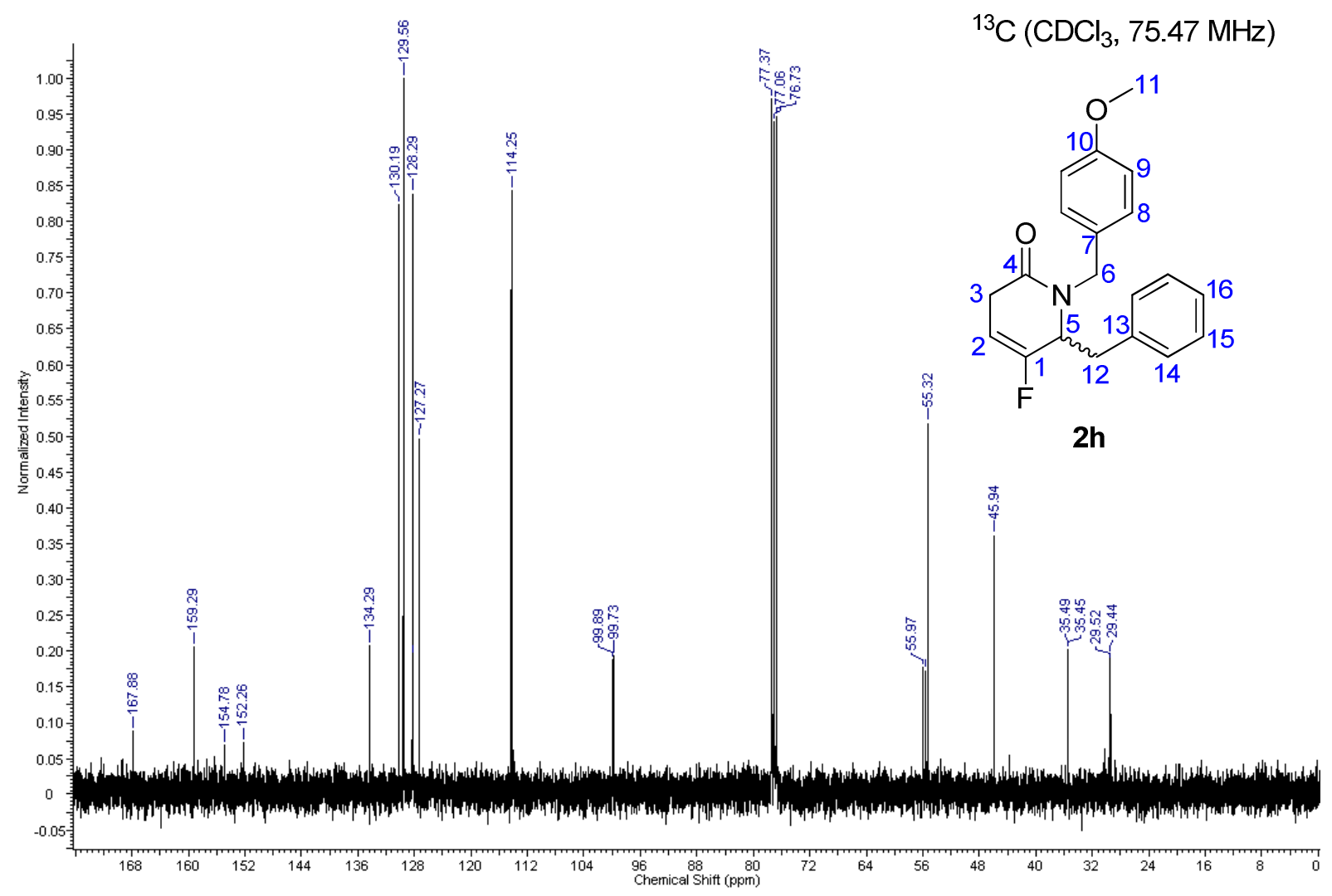

Compound 3b: (S,Z)-N-(3-fluoro-4-phenylbut-3-en-2-yl)-N-(4-methoxybenzyl)but-3-enamide

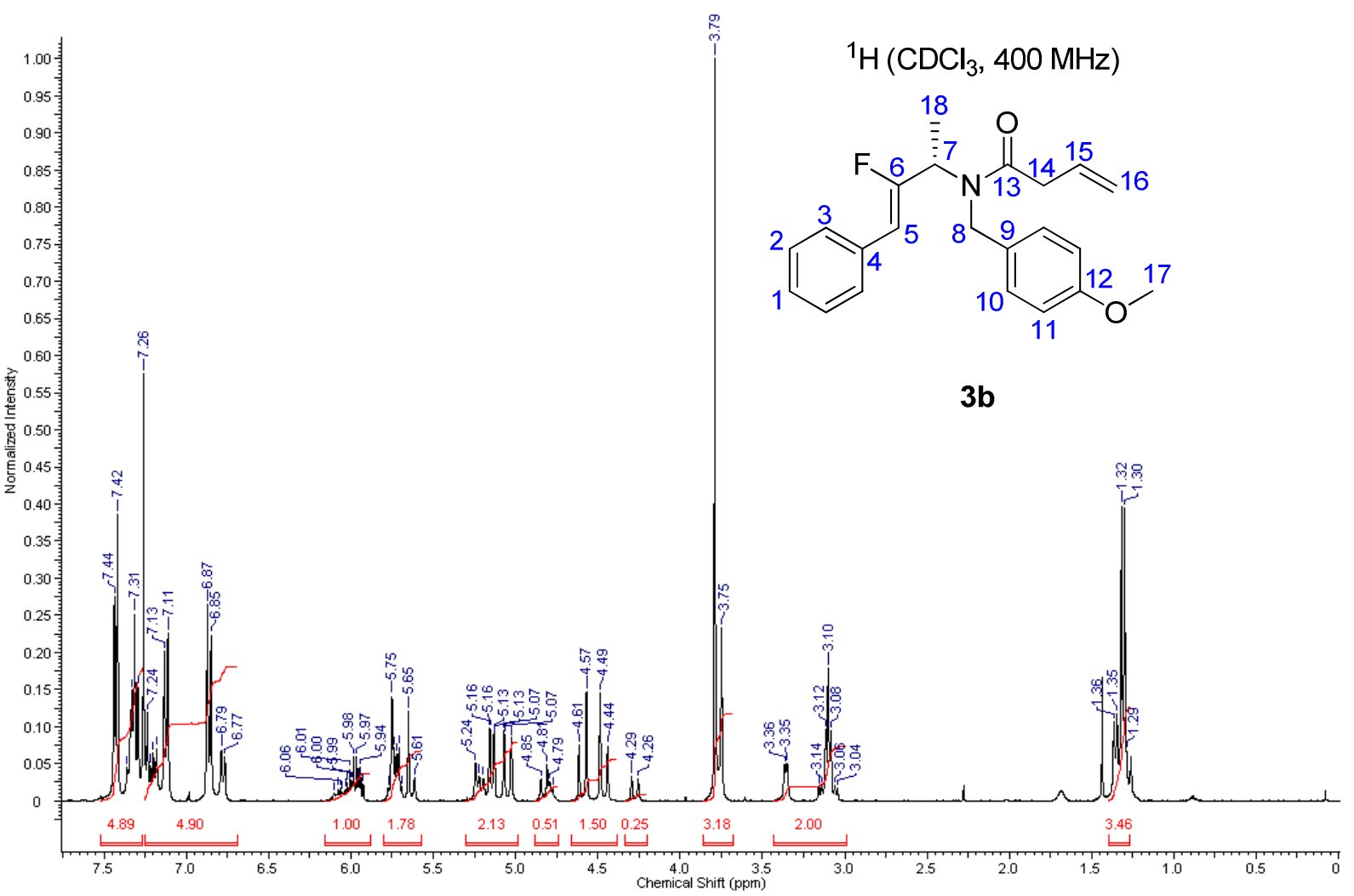



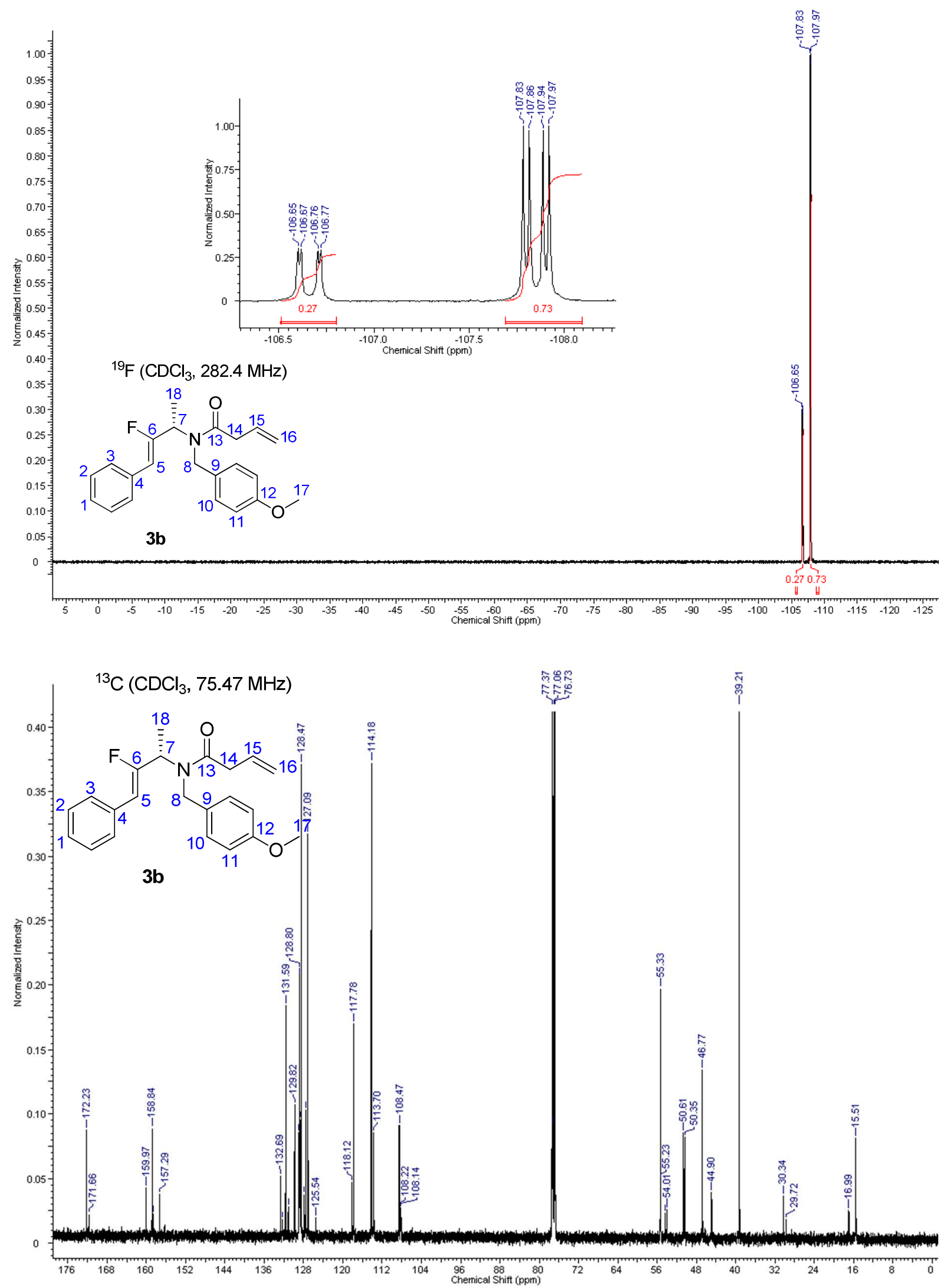

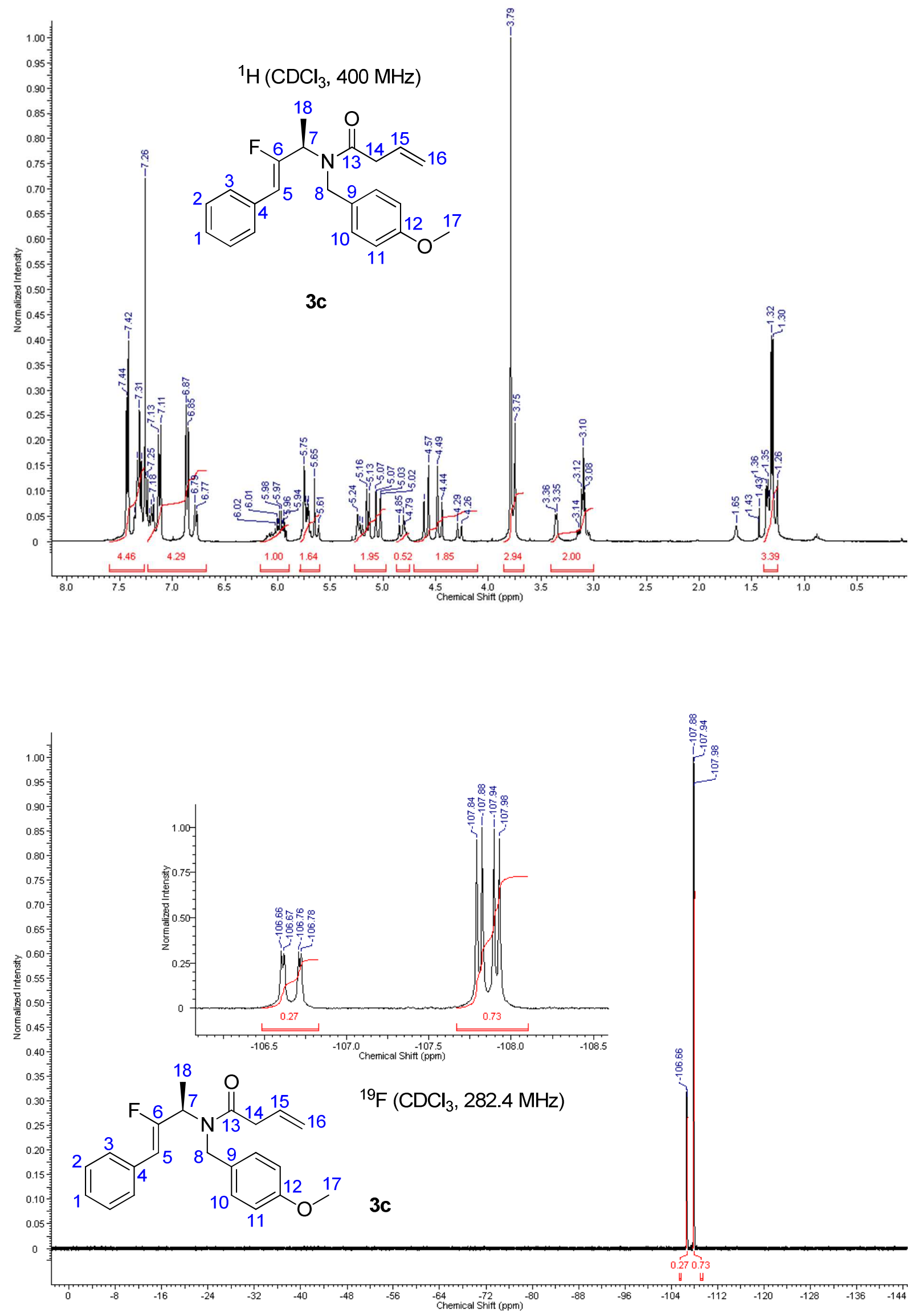


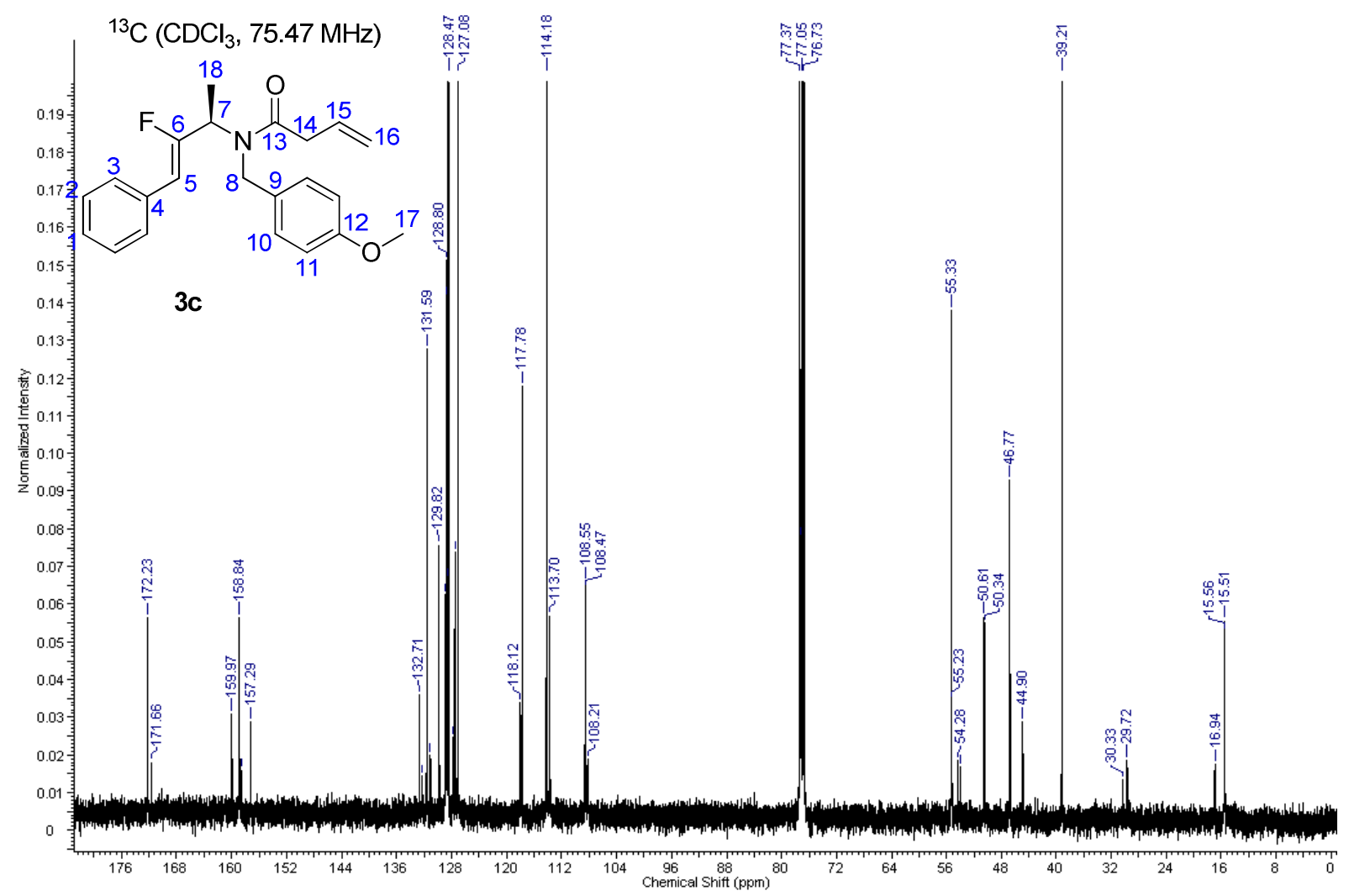

Compound 3d: (S,Z)-N-(2-fluoro-1,3-diphenylallyl)-N-(4-methoxybenzyl)but-3-enamide

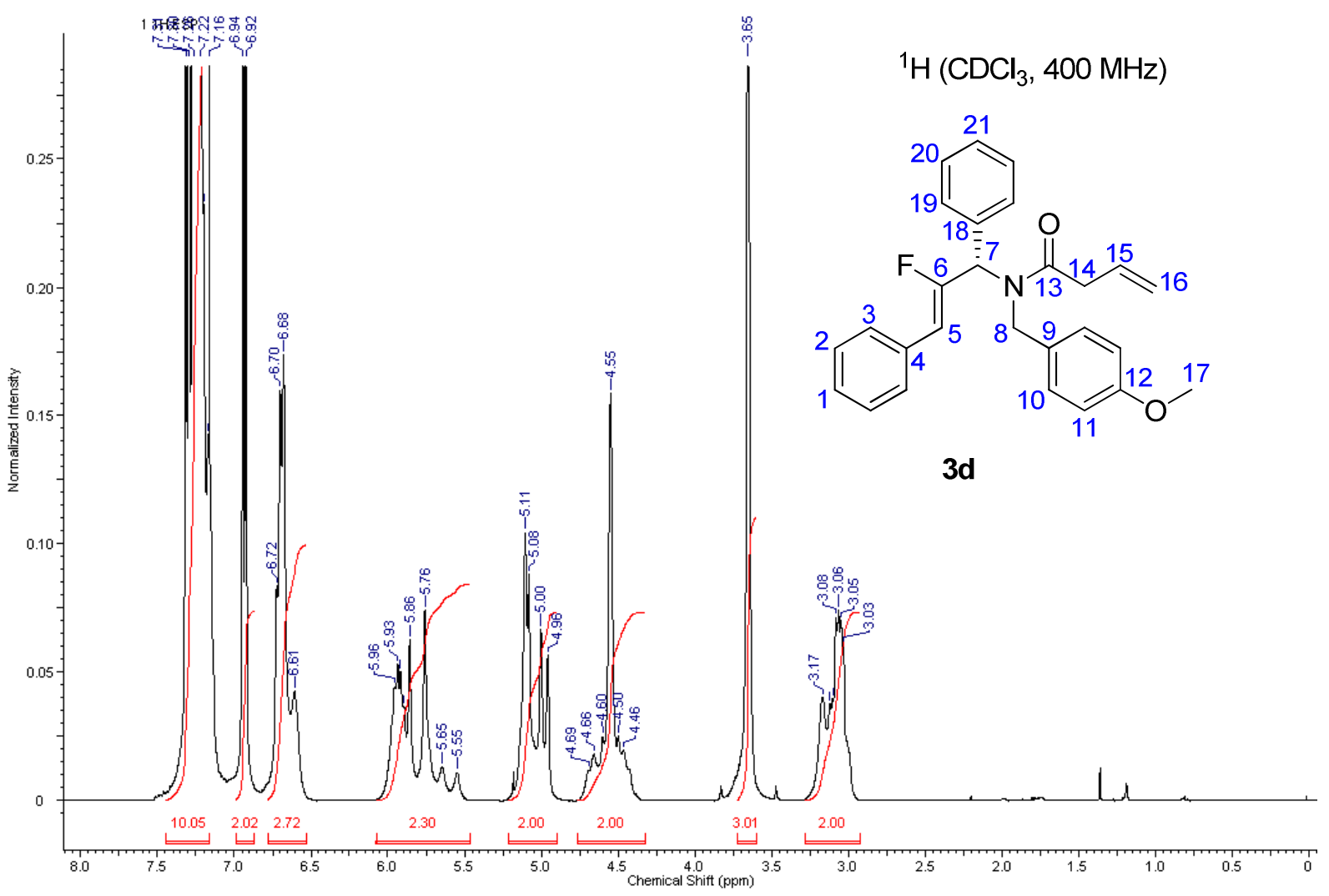




$$
\text { 페 }
$$


Compound 3d+e: Racemic (Z)-N-(2-fluoro-1,3-diphenylallyl)-N-(4-methoxybenzyl)but-3-

enamide
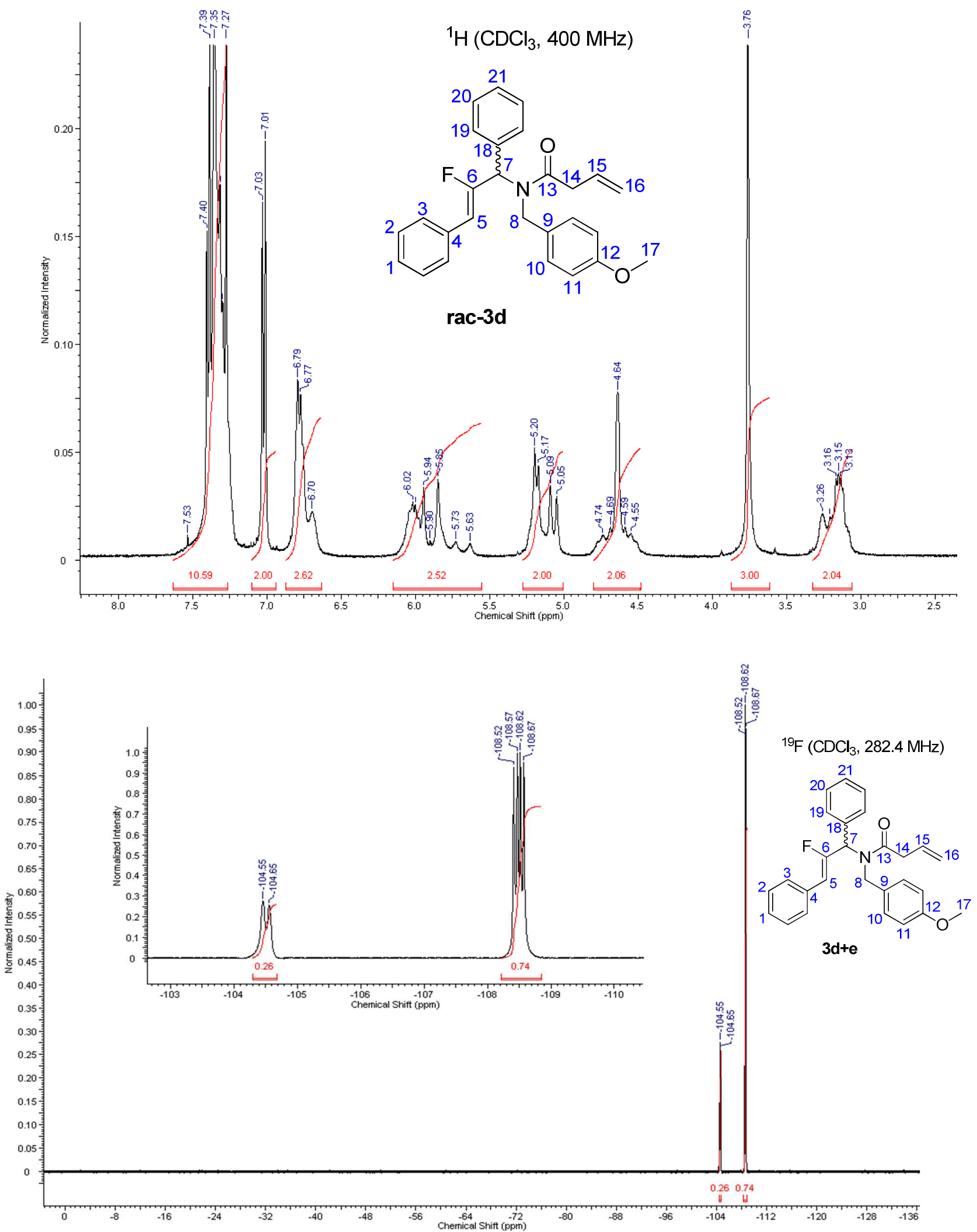


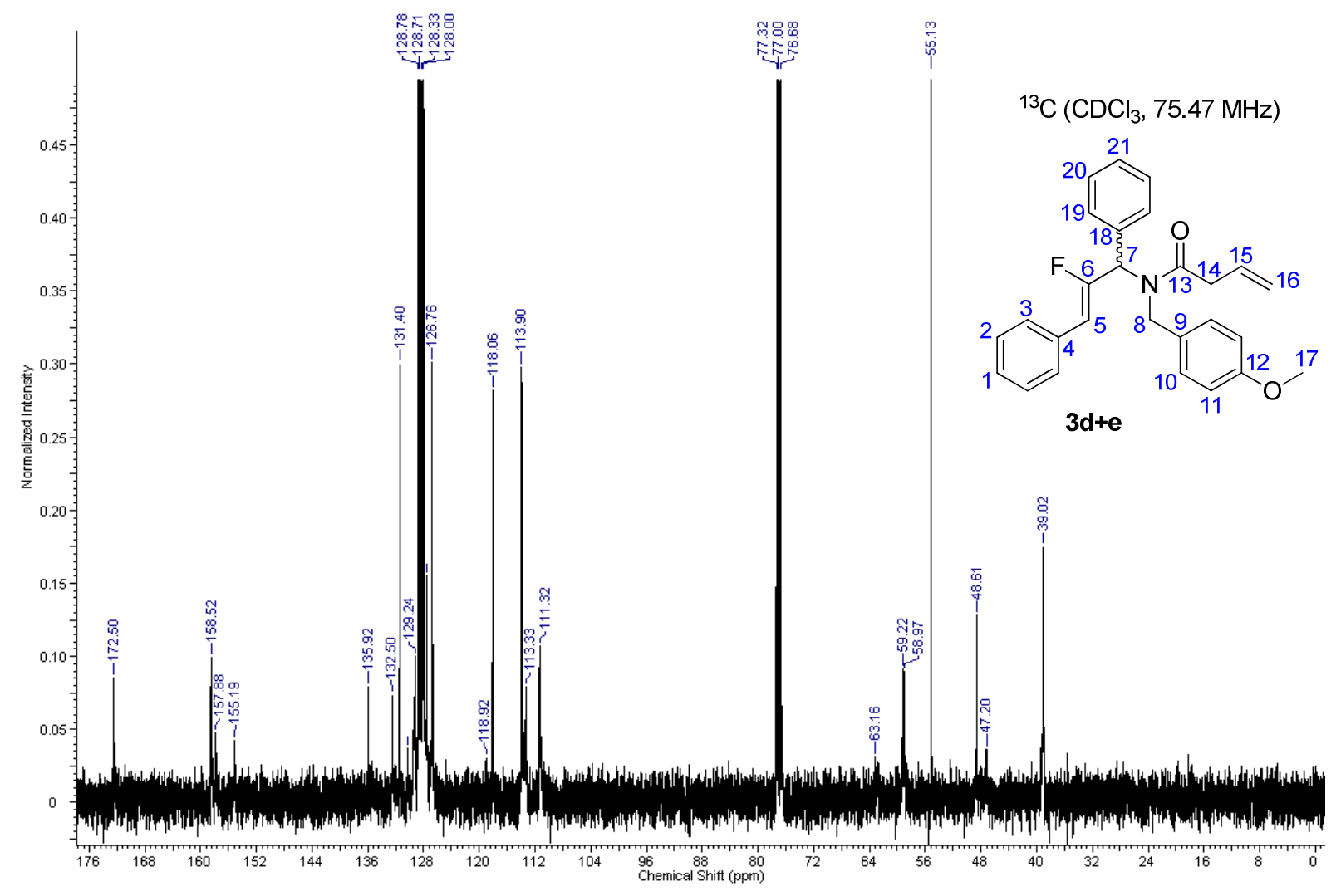

Compound 3f: (S,Z)-N-(2-fluoro-4-methyl-1-phenylpent-1-en-3-yl)-N-(4-methoxybenzyl)but-3enamide

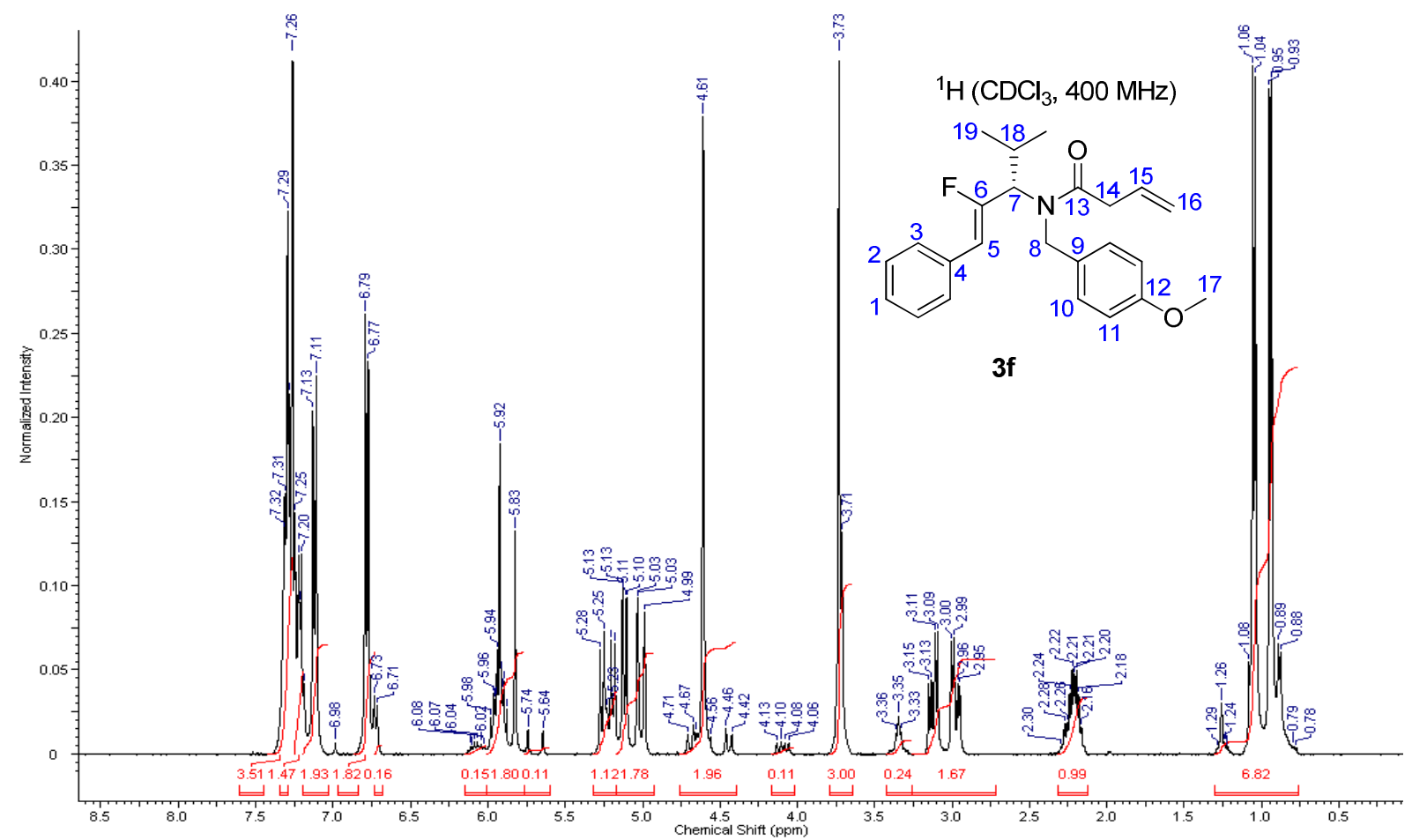



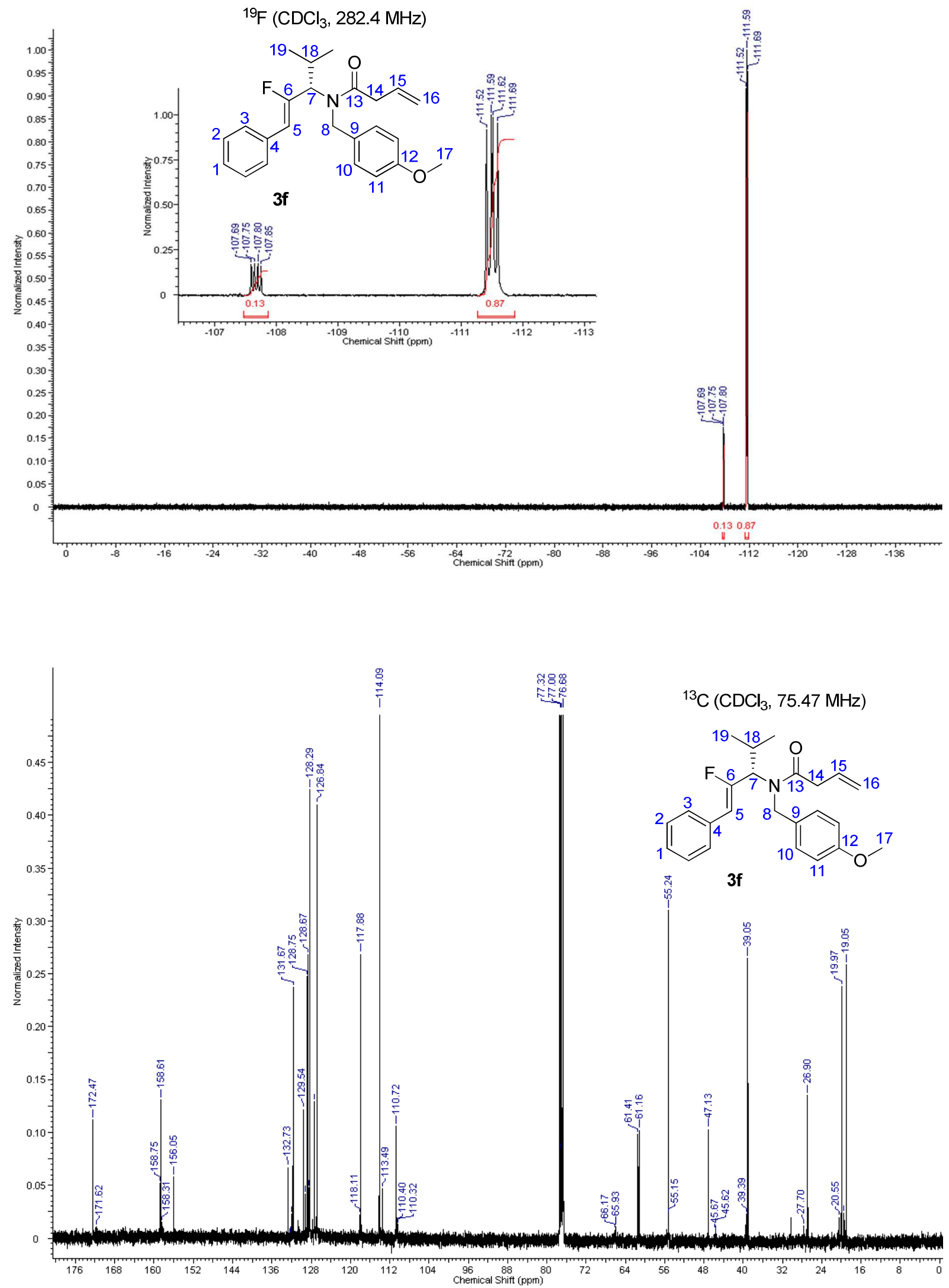
Compound 3g: (R,Z)-N-(2-fluoro-4-methyl-1-phenylpent-1-en-3-yl)-N-(4-methoxybenzyl)but-3enamide
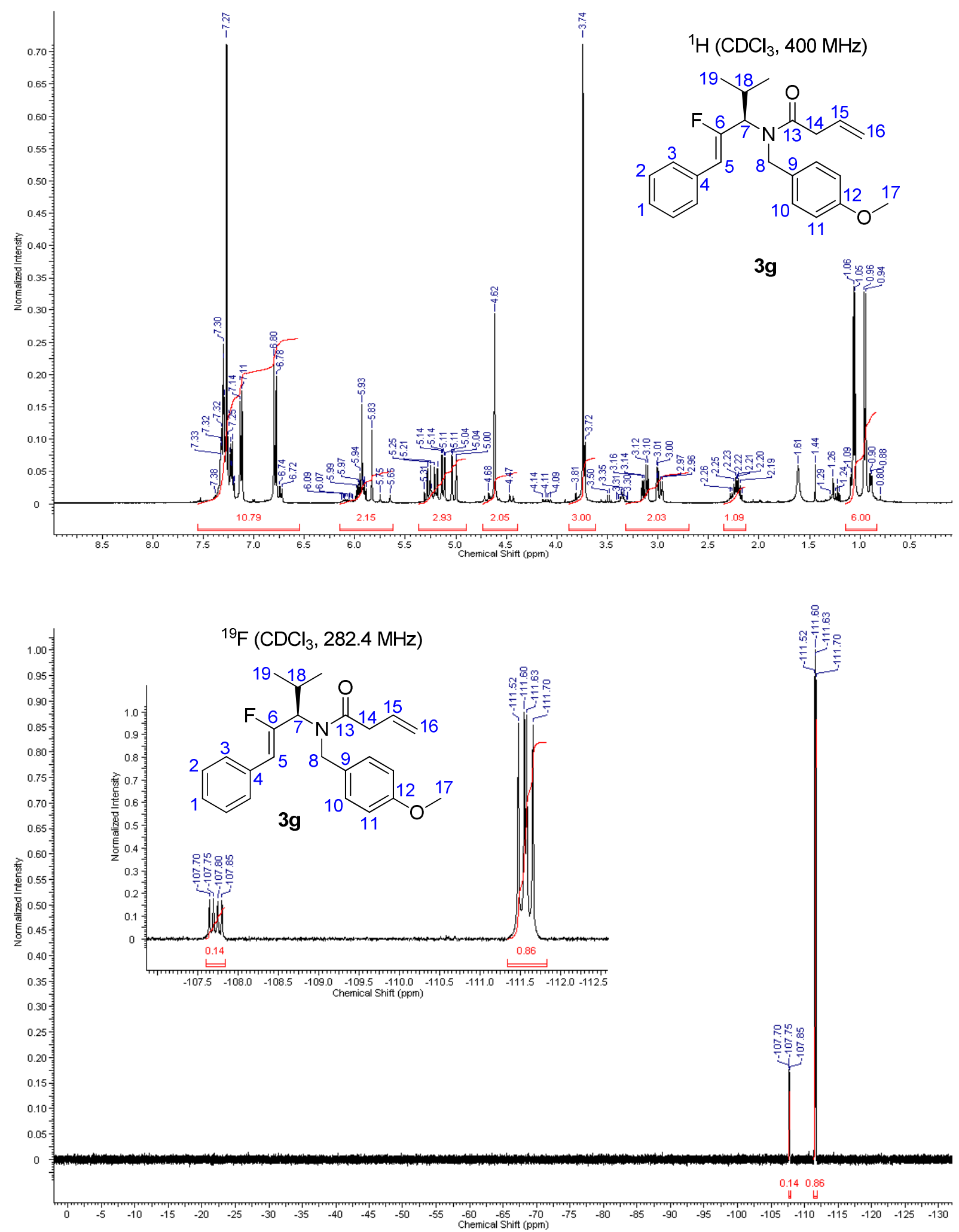


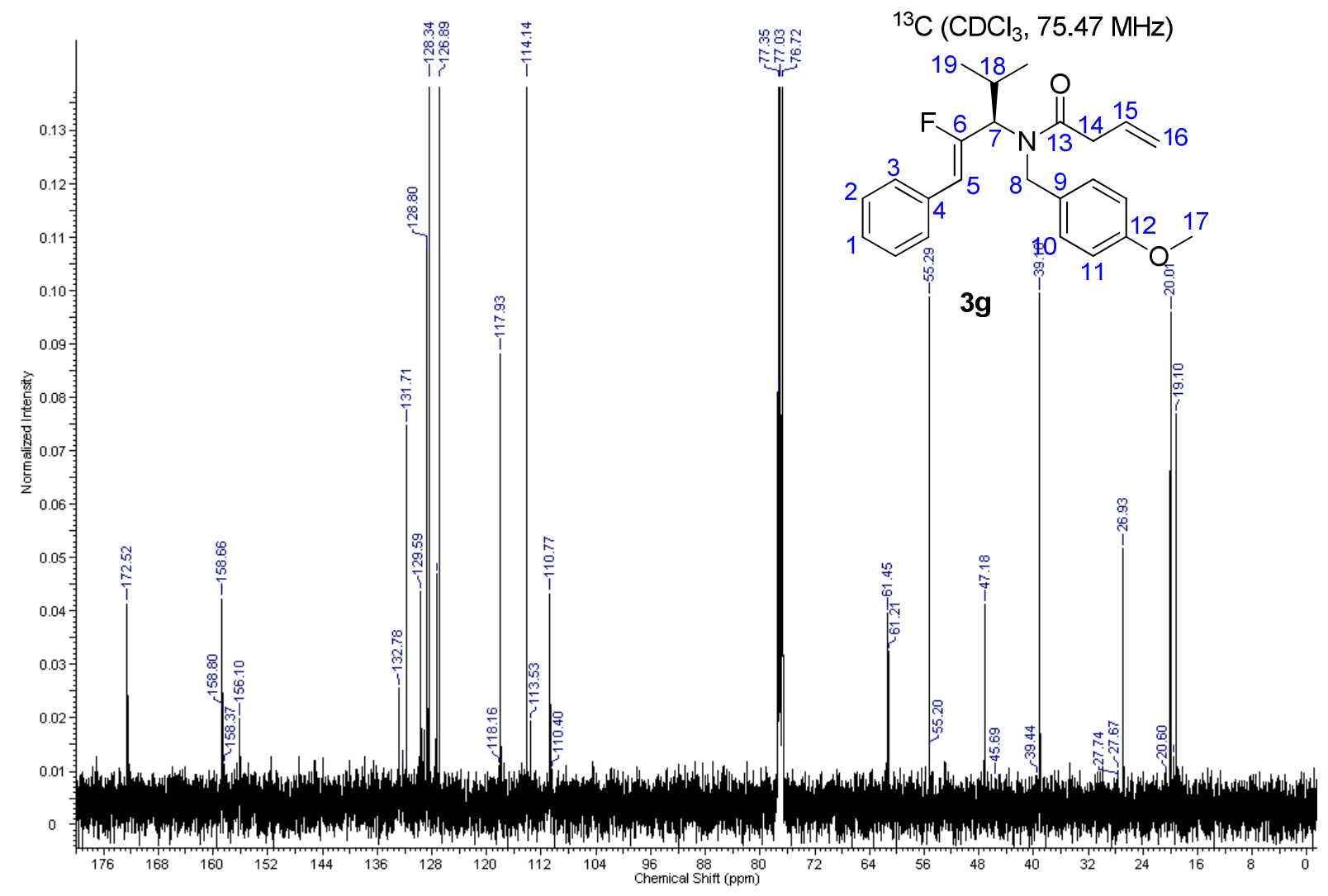

Compound 3h: (S,Z)-N-(3-fluoro-1,4-diphenylbut-3-en-2-yl)-N-(4-methoxybenzyl)but-3enamide

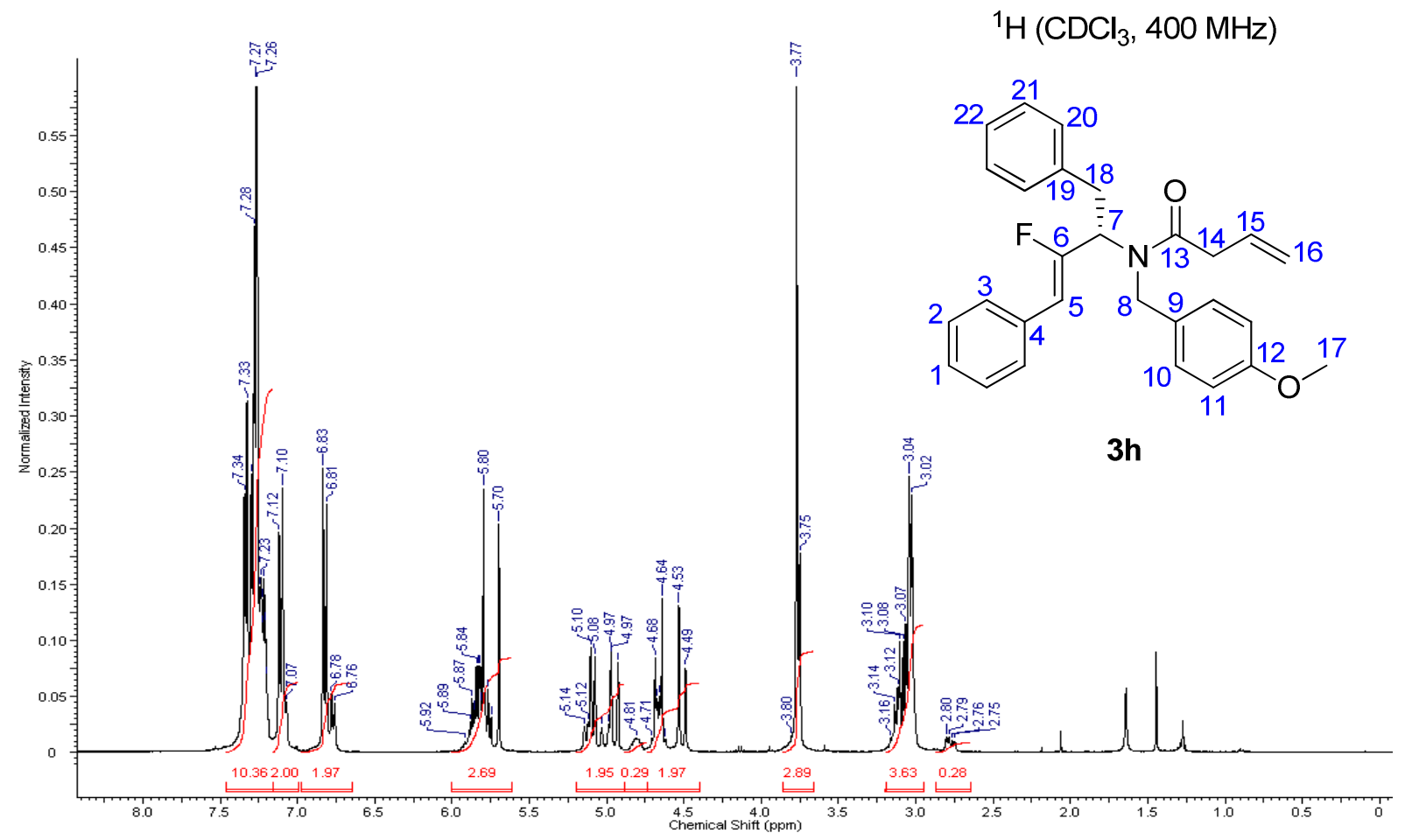



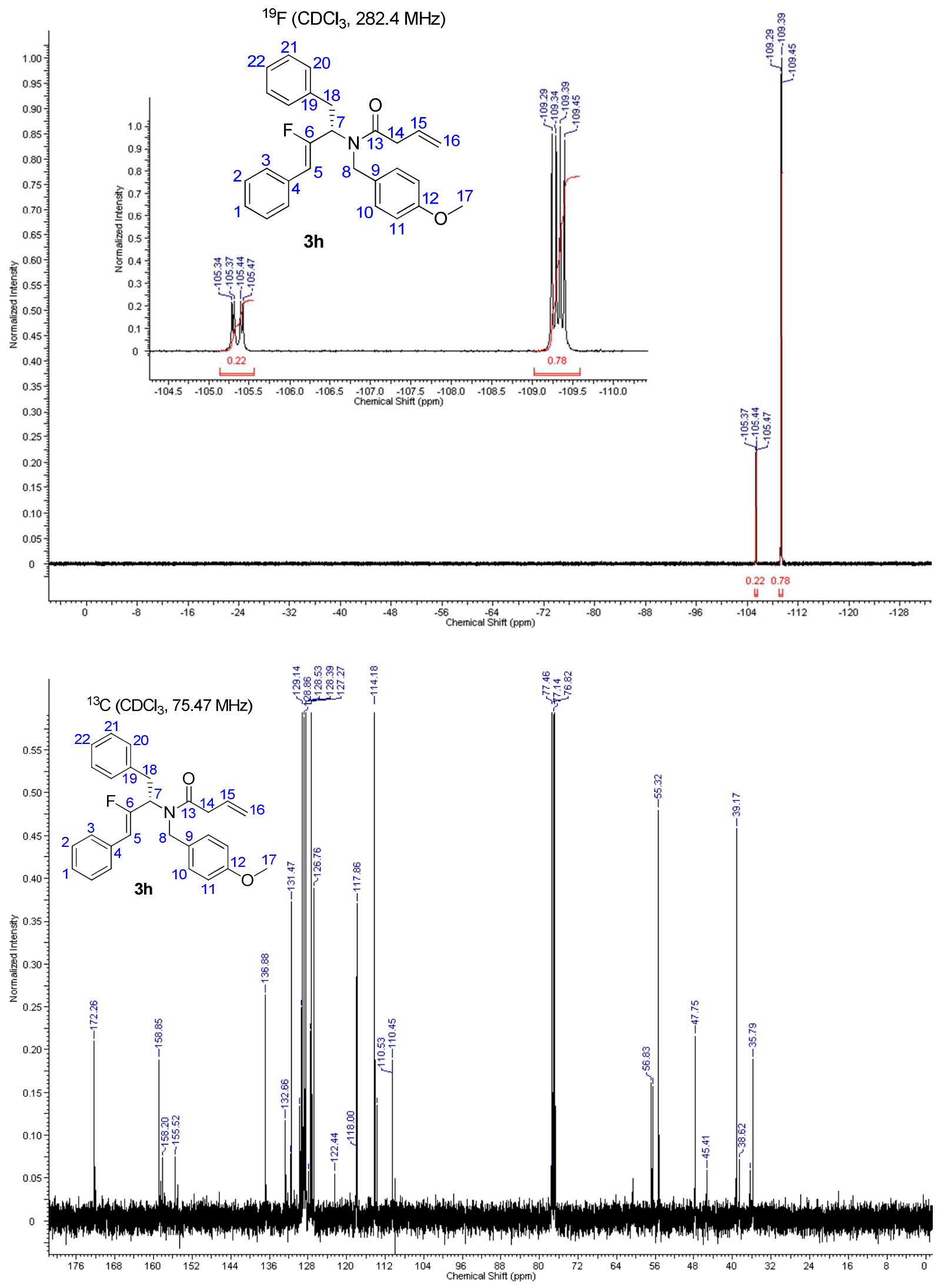
Compound 4b: (R)-N-((S,Z)- 3-fluoro-4-phenylbut-3-en-2-yl)-2-methylpropane-2-sulfinamide
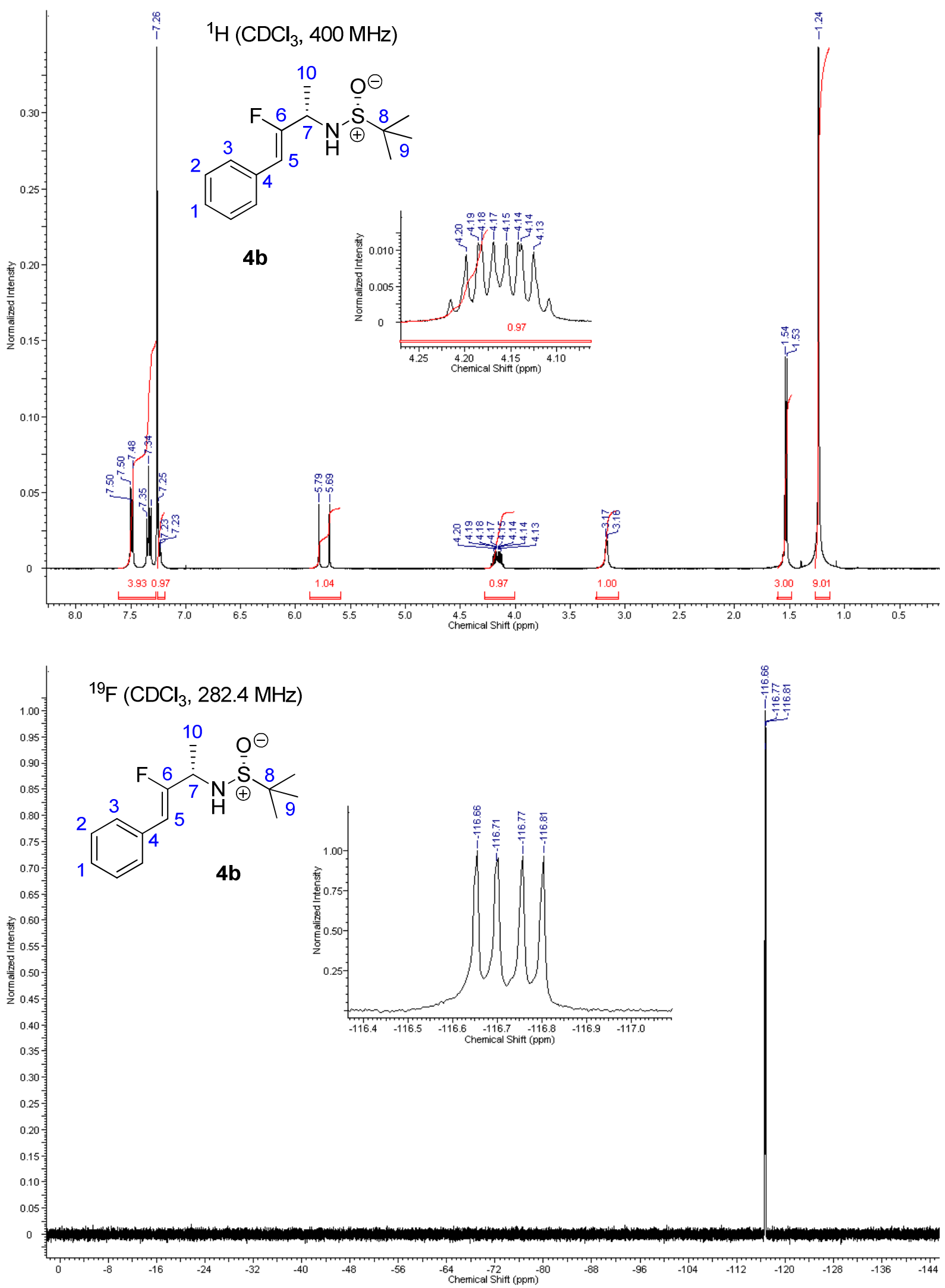


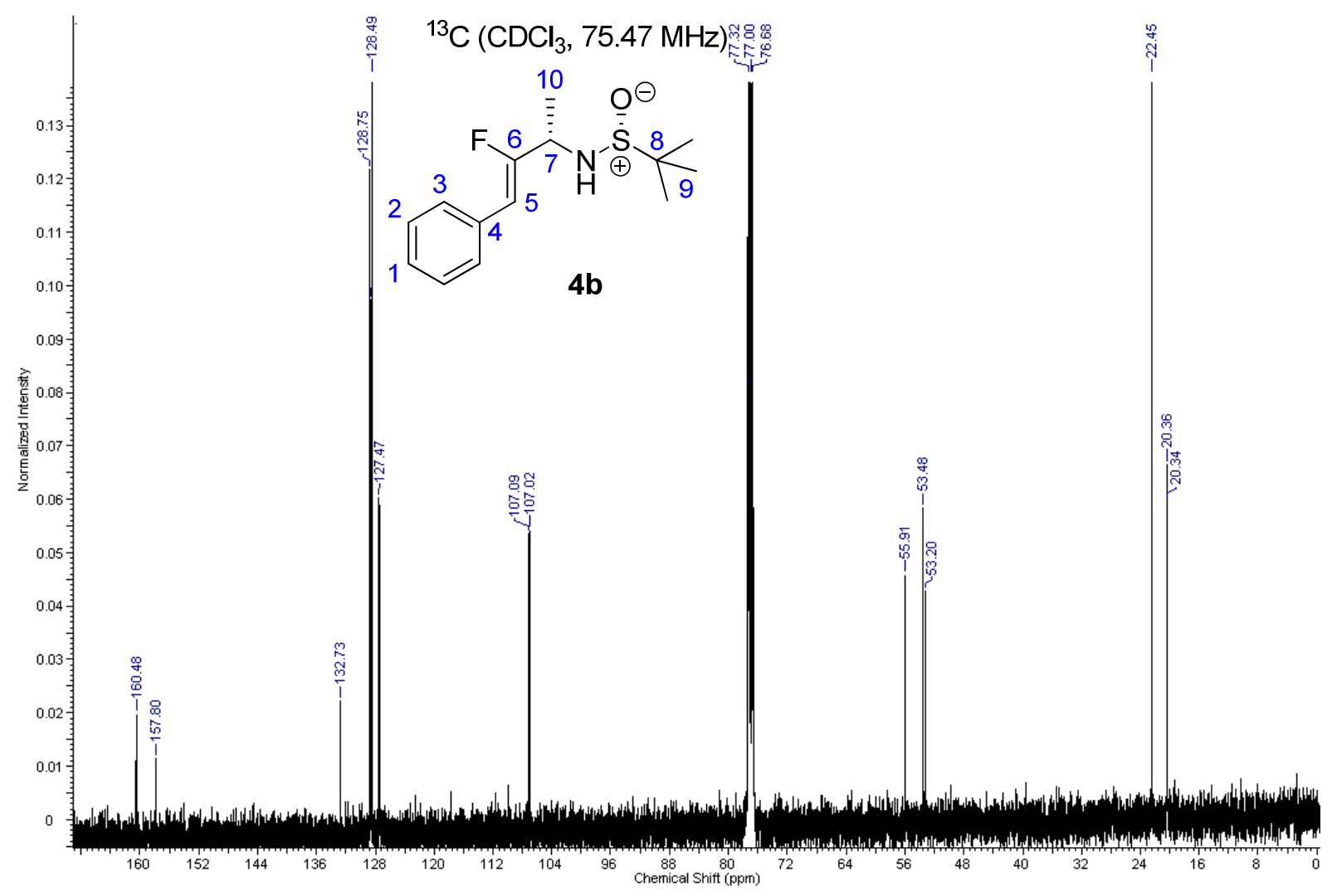

Compound 4c: (R)-N-((R,Z)- 3-fluoro-4-phenylbut-3-en-2-yl)-2-methylpropane-2-sulfinamide

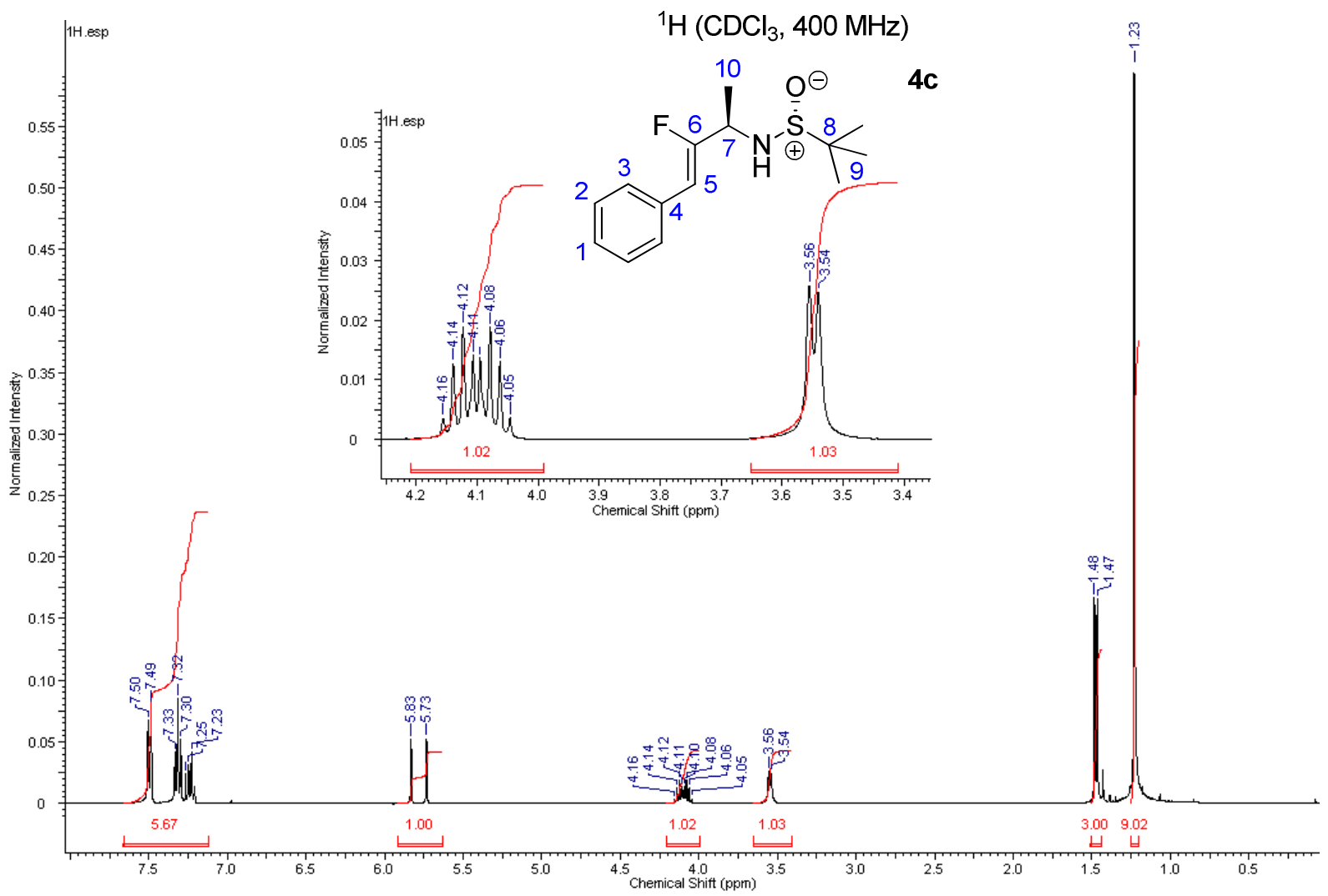



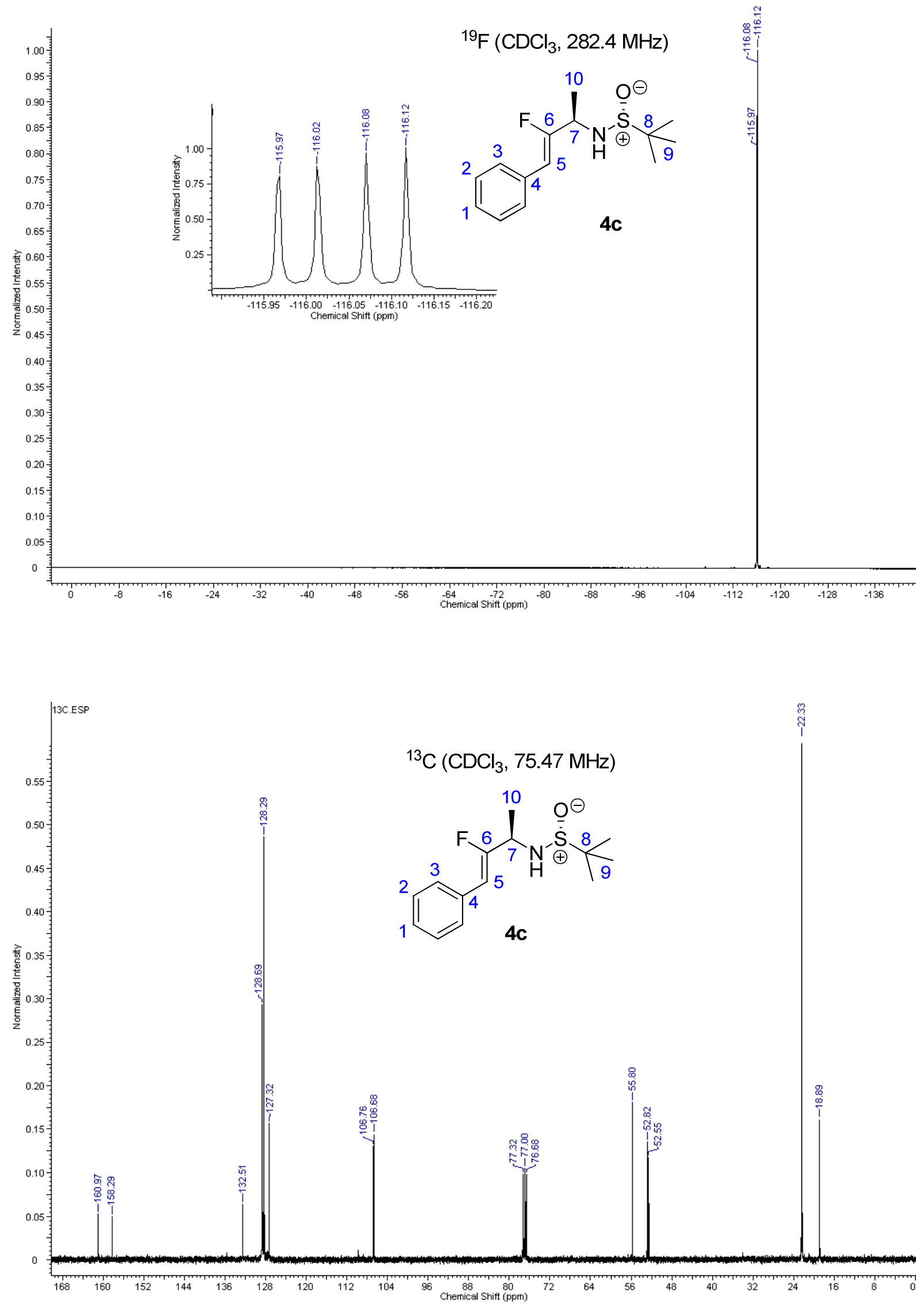
Compound 4d: (R)-N-((S,Z)- 2-fluoro-1,3-diphenylallyl)-2-methylpropane-2-sulfinamide
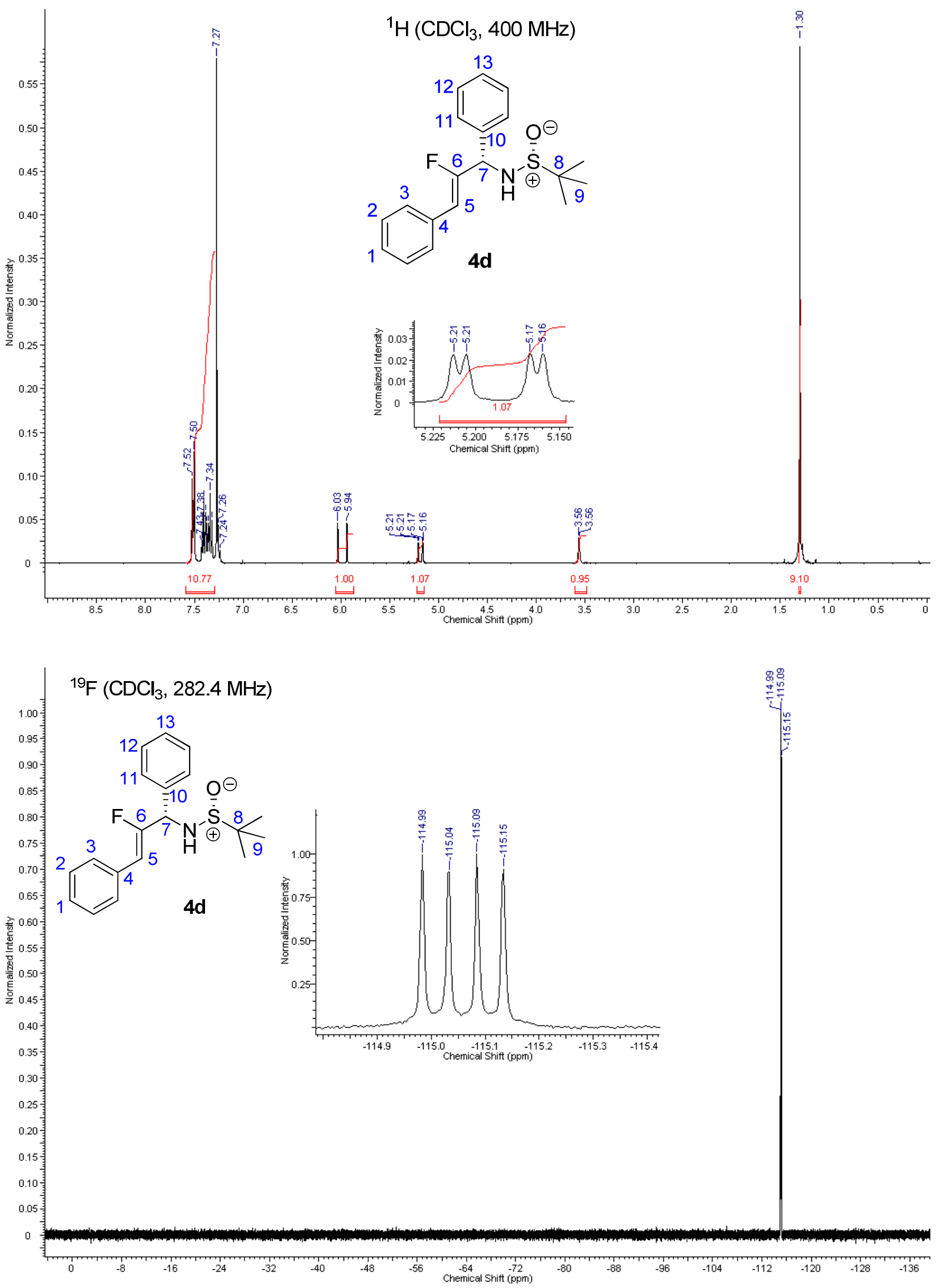


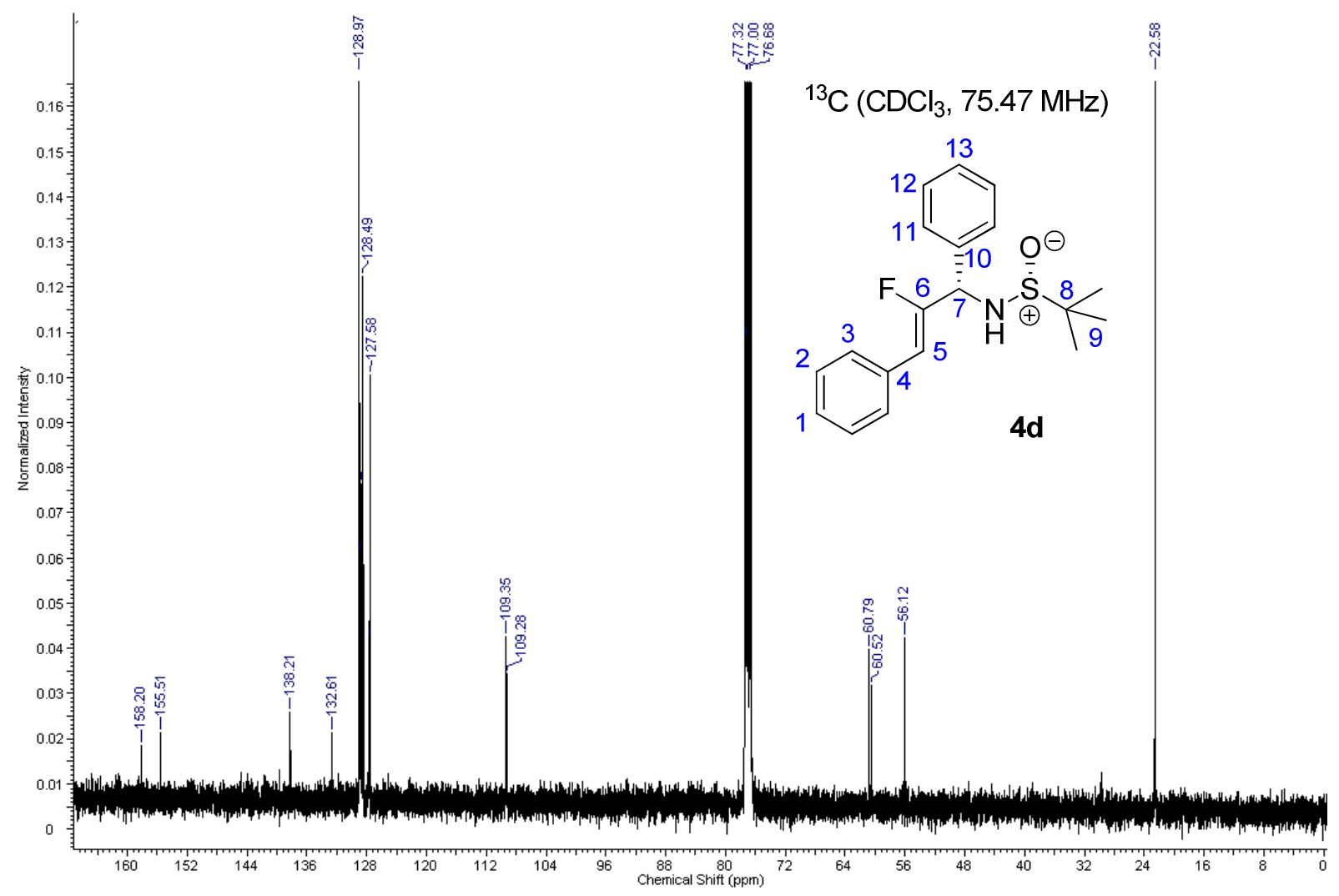

Compound 4d+e: $N$-((R,Z)-2-fluoro-1,3-diphenylallyl)-2-methylpropane-2-sulfinamide

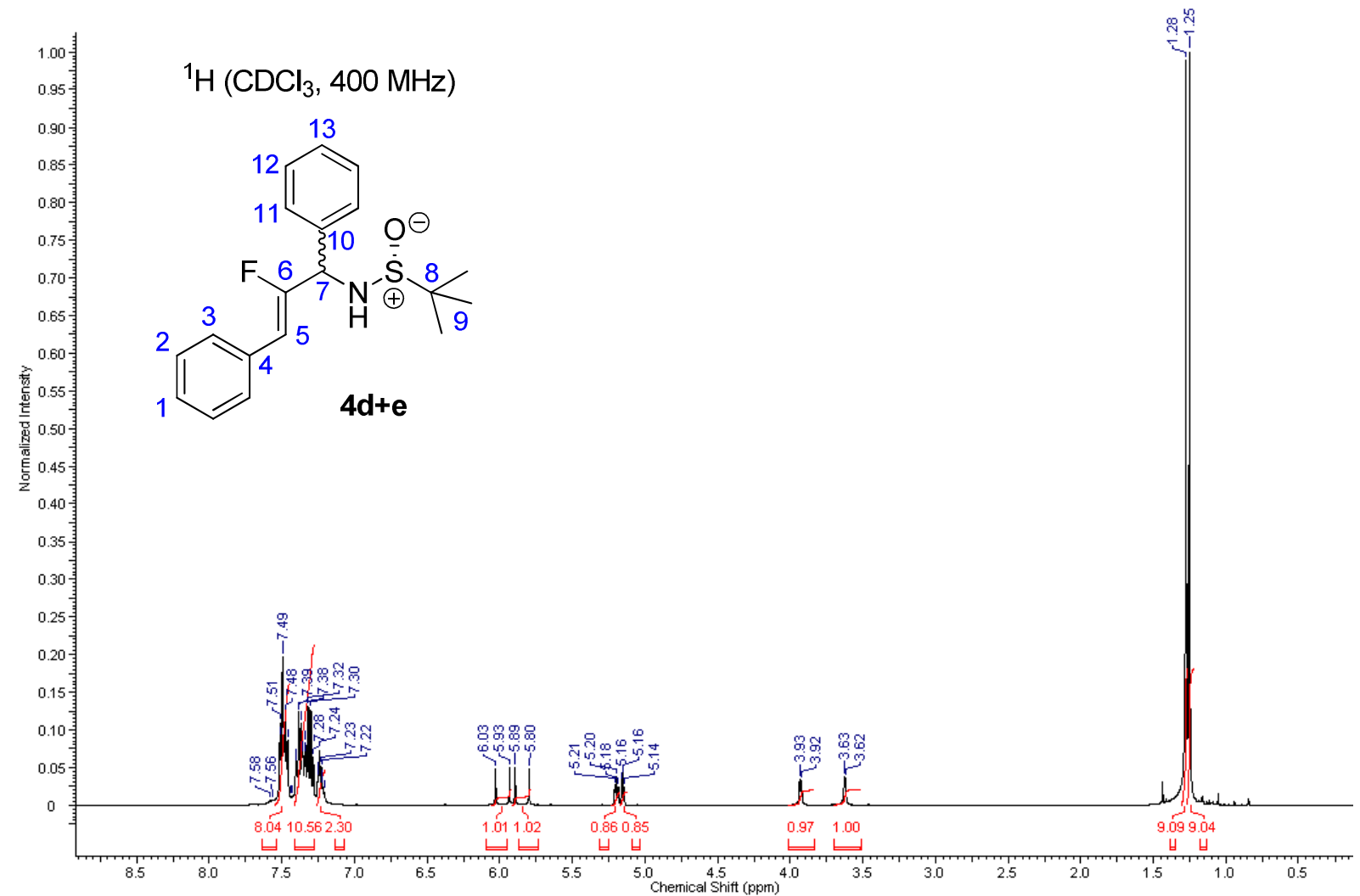




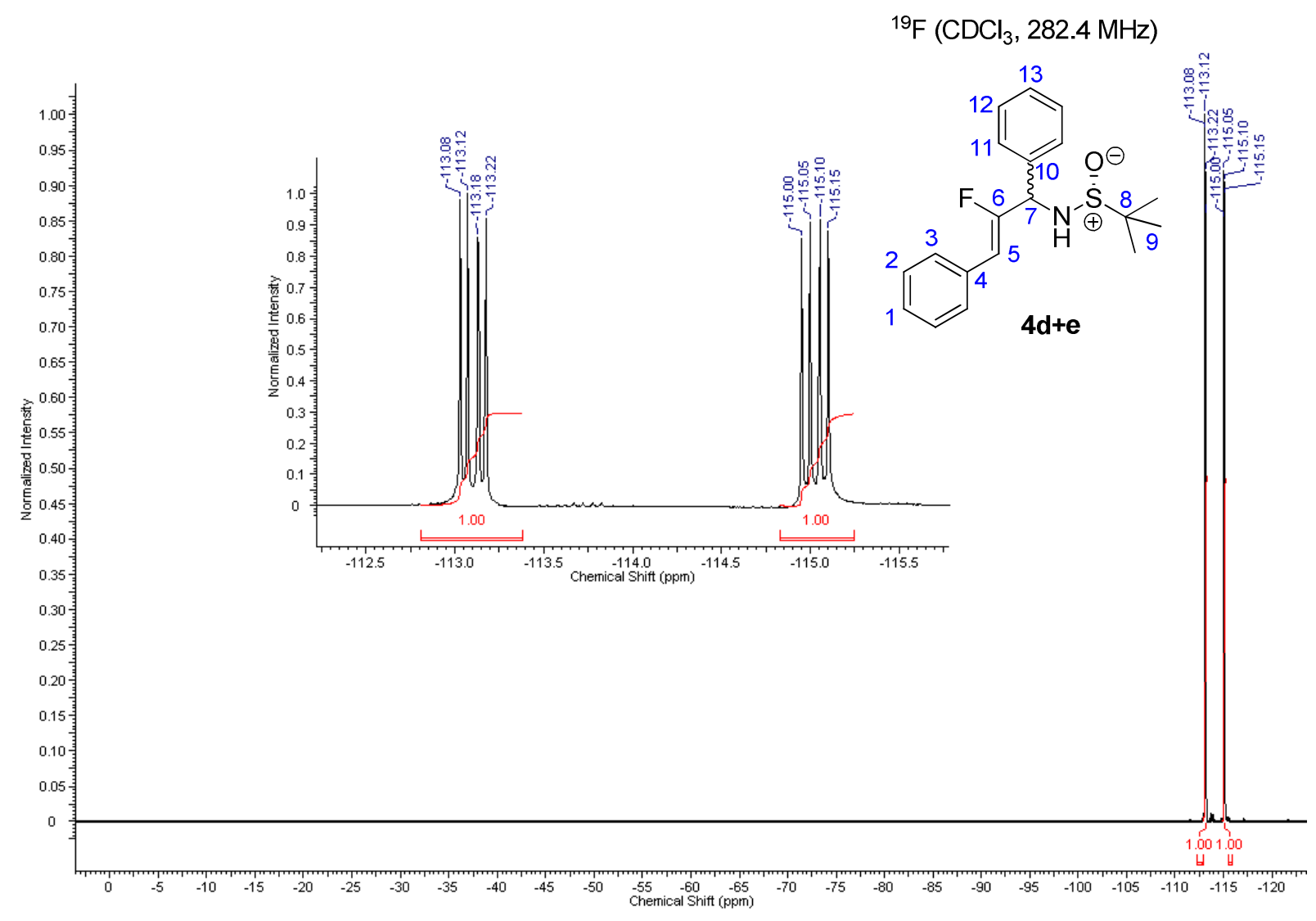

Compound 4f: (Rs)-N-((S,Z)-2-fluoro-4-methyl-1-phenylpent-1-en-3-yl)-2-methylpropane-2sulfinamide

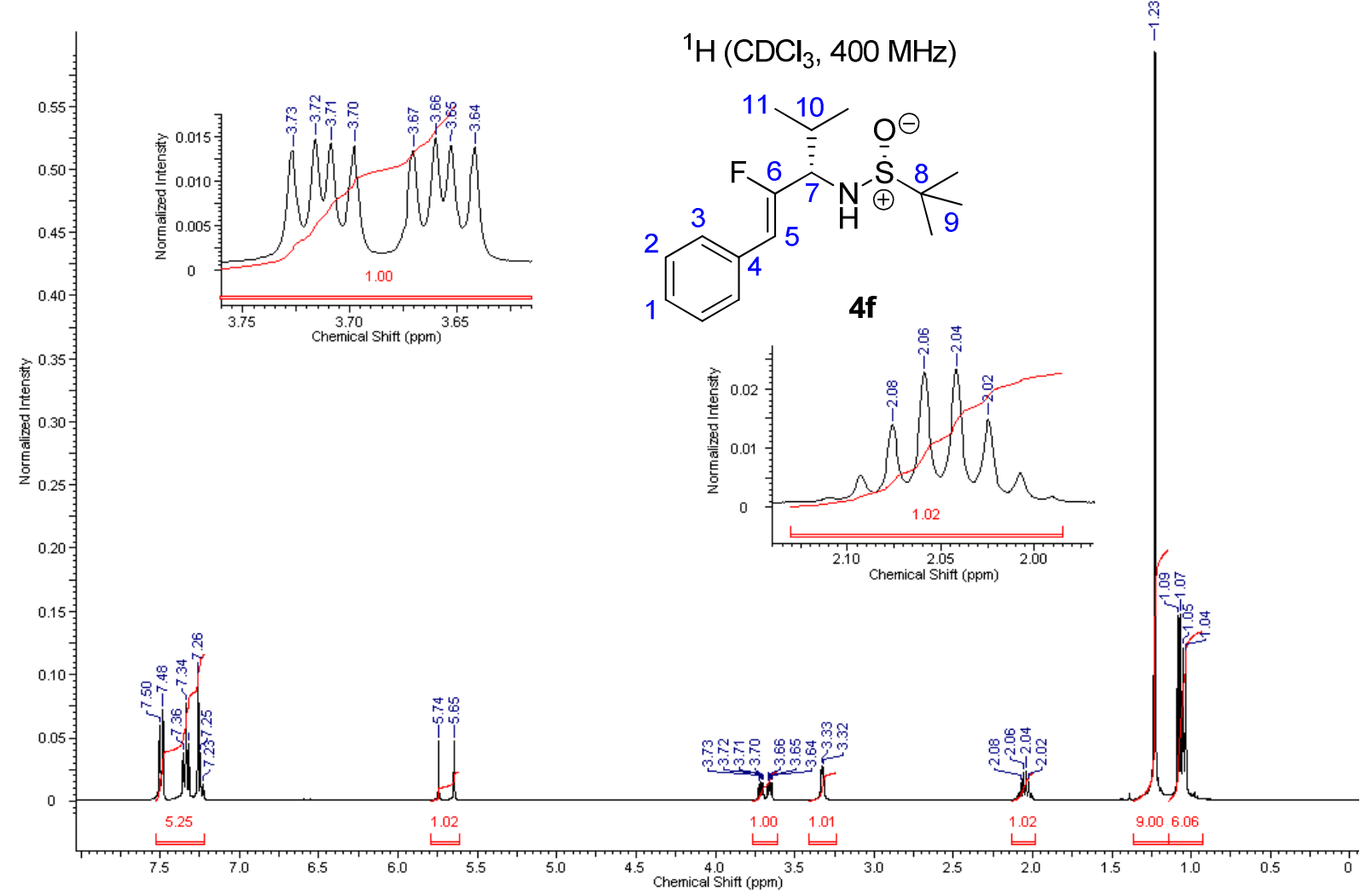



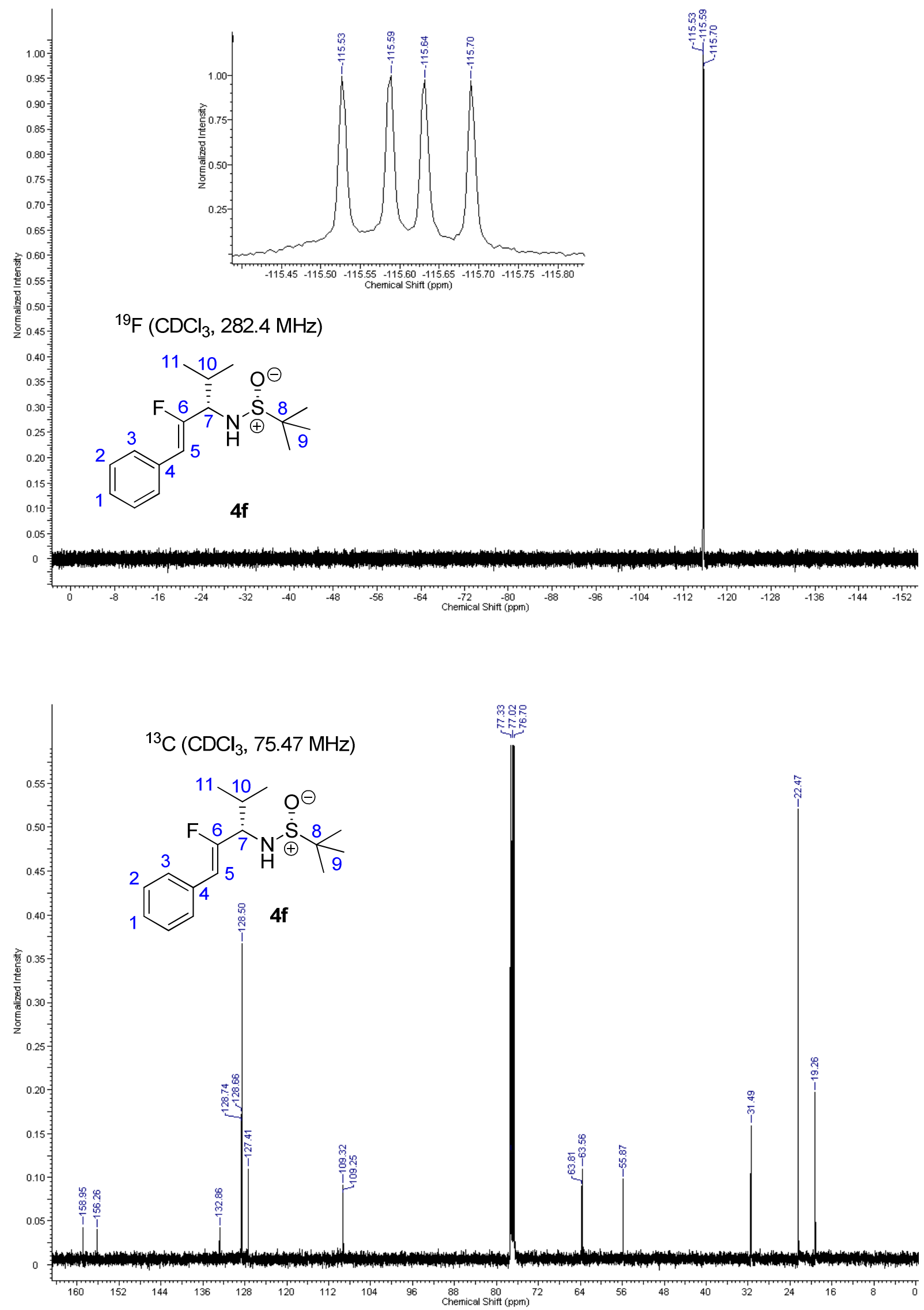
Compound 4g: (Rs)-N-((Z)-2-fluoro-4-methyl-1-phenylpent-1-en-3-yl)-2-methylpropane-2sulfinamide
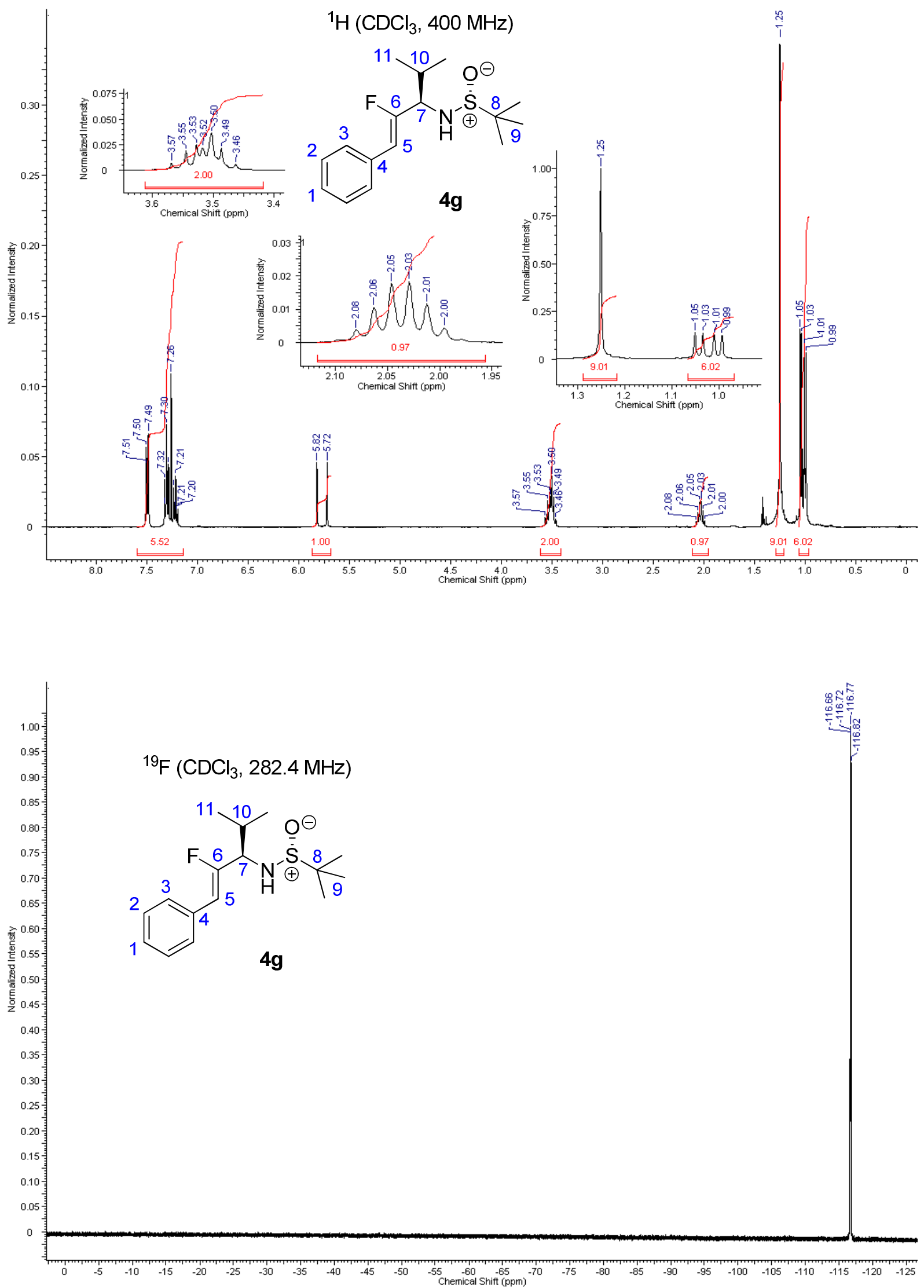

S61 


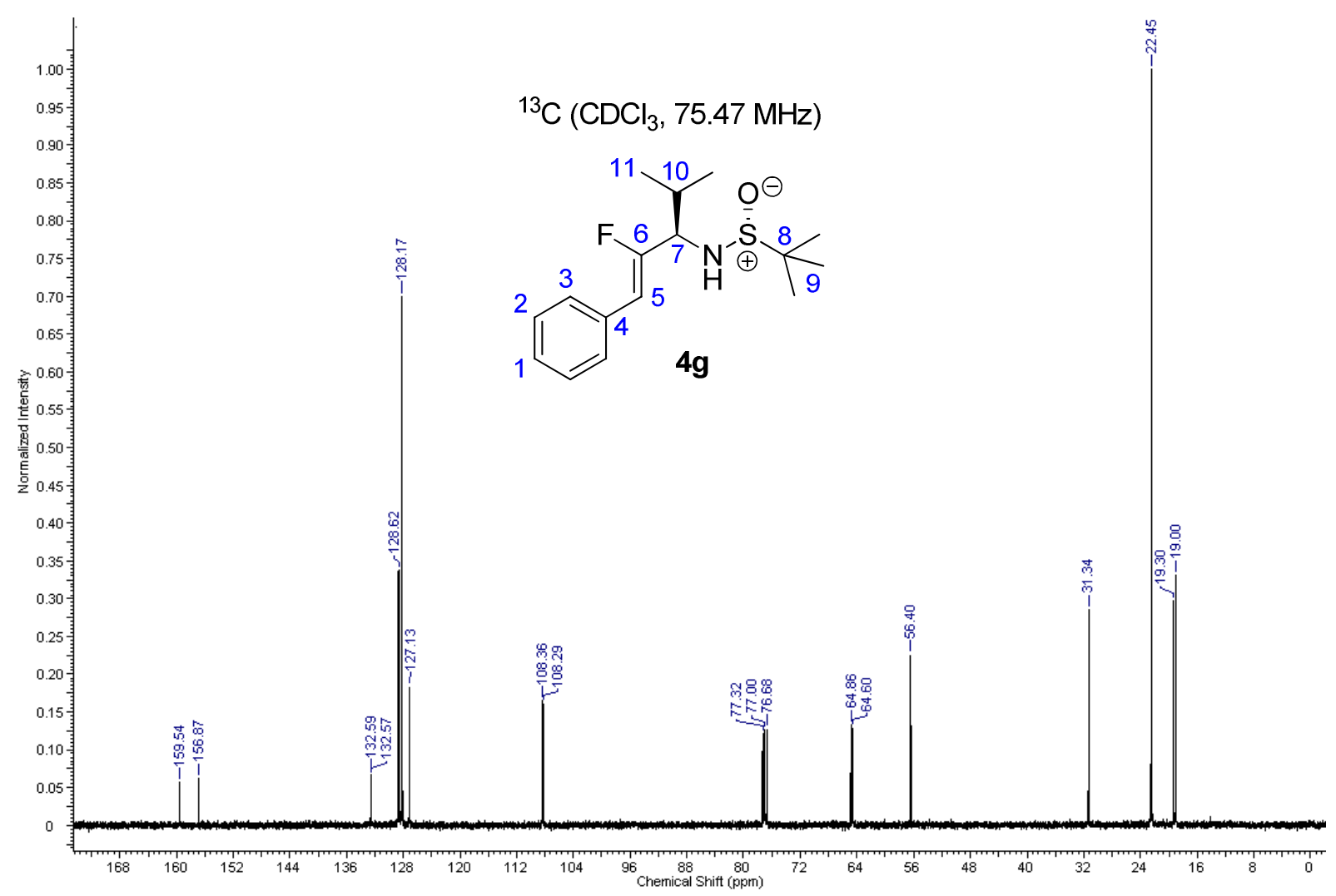

Compound 4h: (Z)-N-(3-fluoro-1,4-diphenylbut-3-en-2-yl)-2-methylpropane-2-sulfinamide

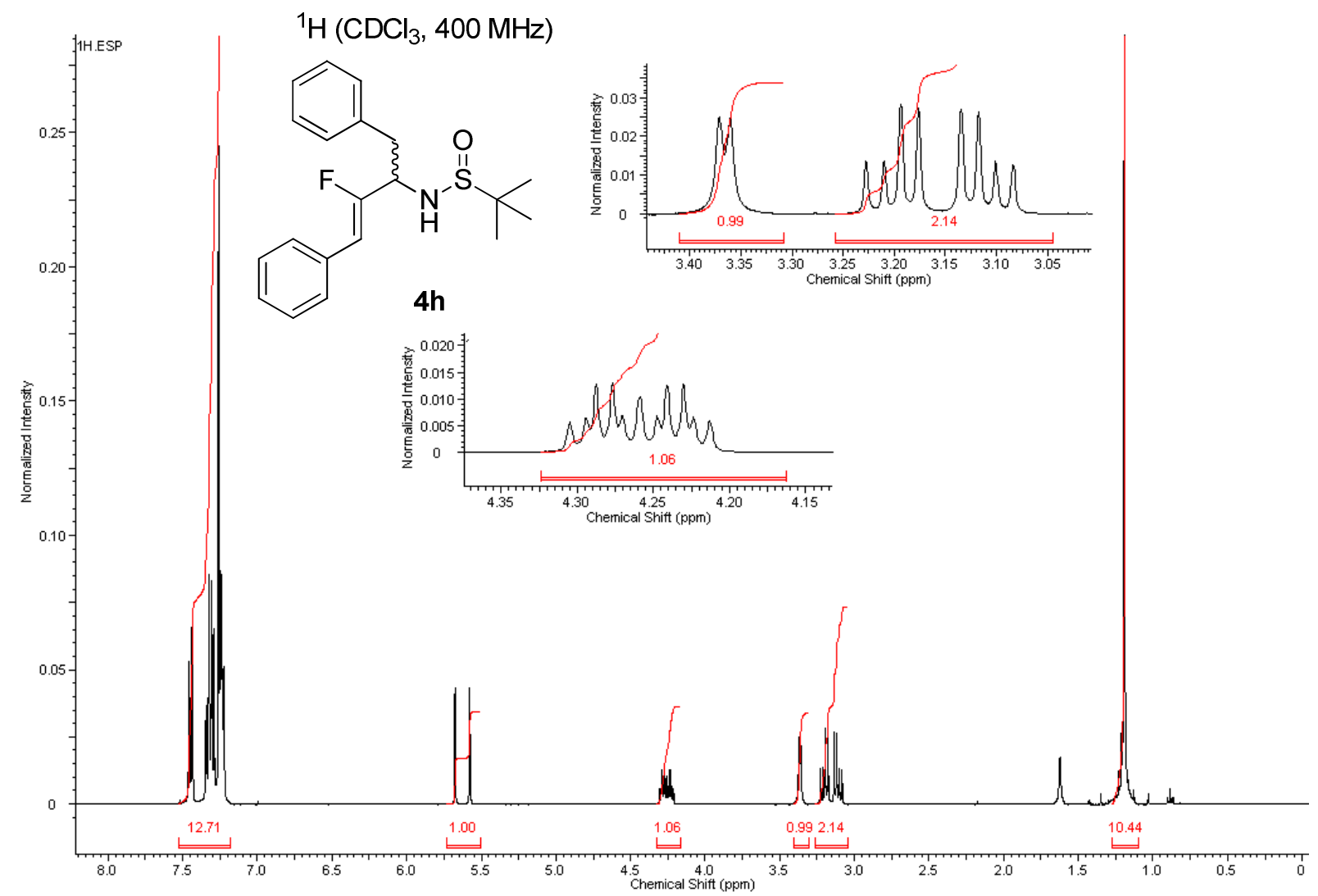



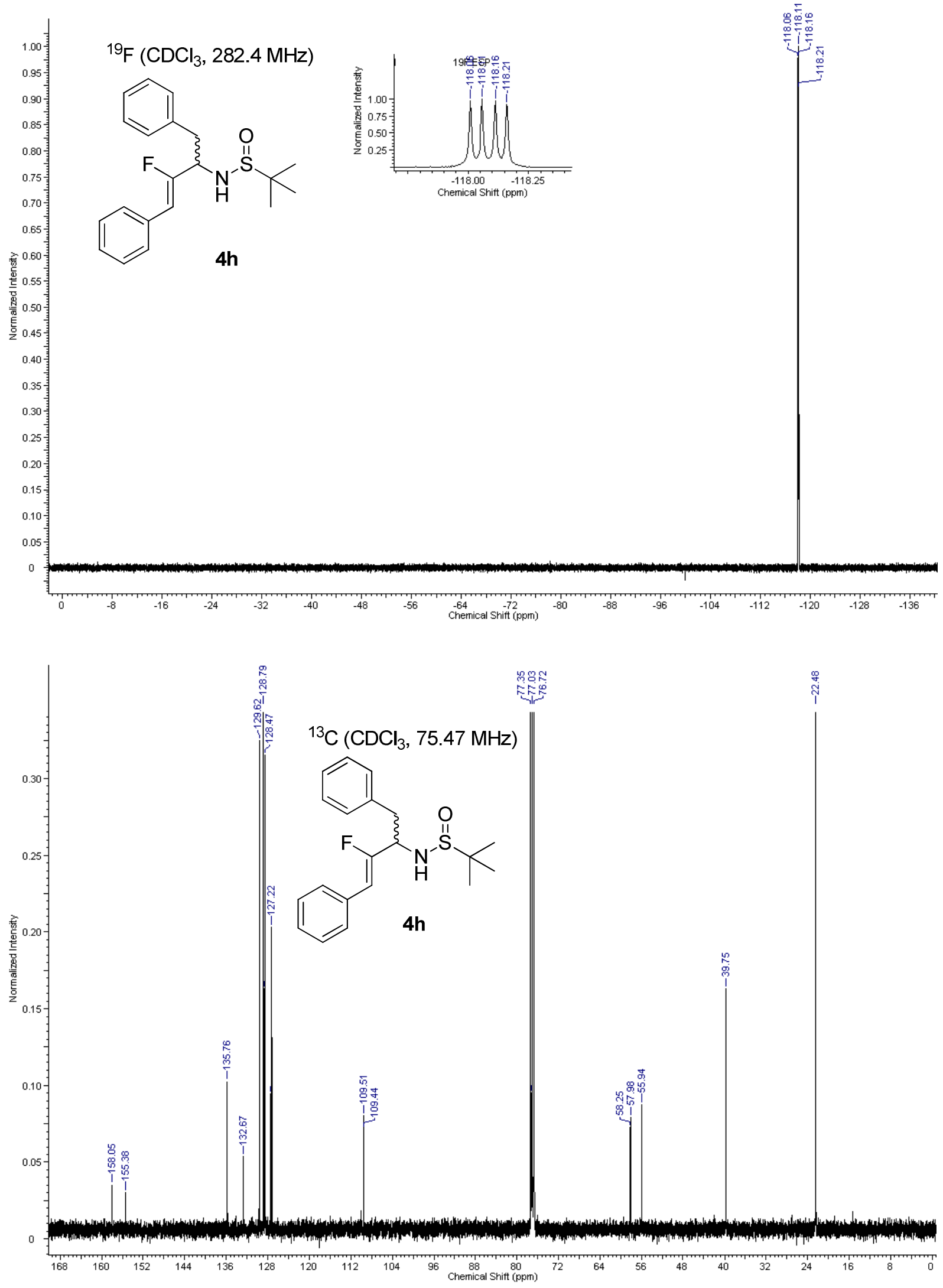

S63 
Compound 5-R: (R,E)-N-((Z)-2-fluoro-3-phenylallylidene)-2-methylpropane-2-sulfinamide
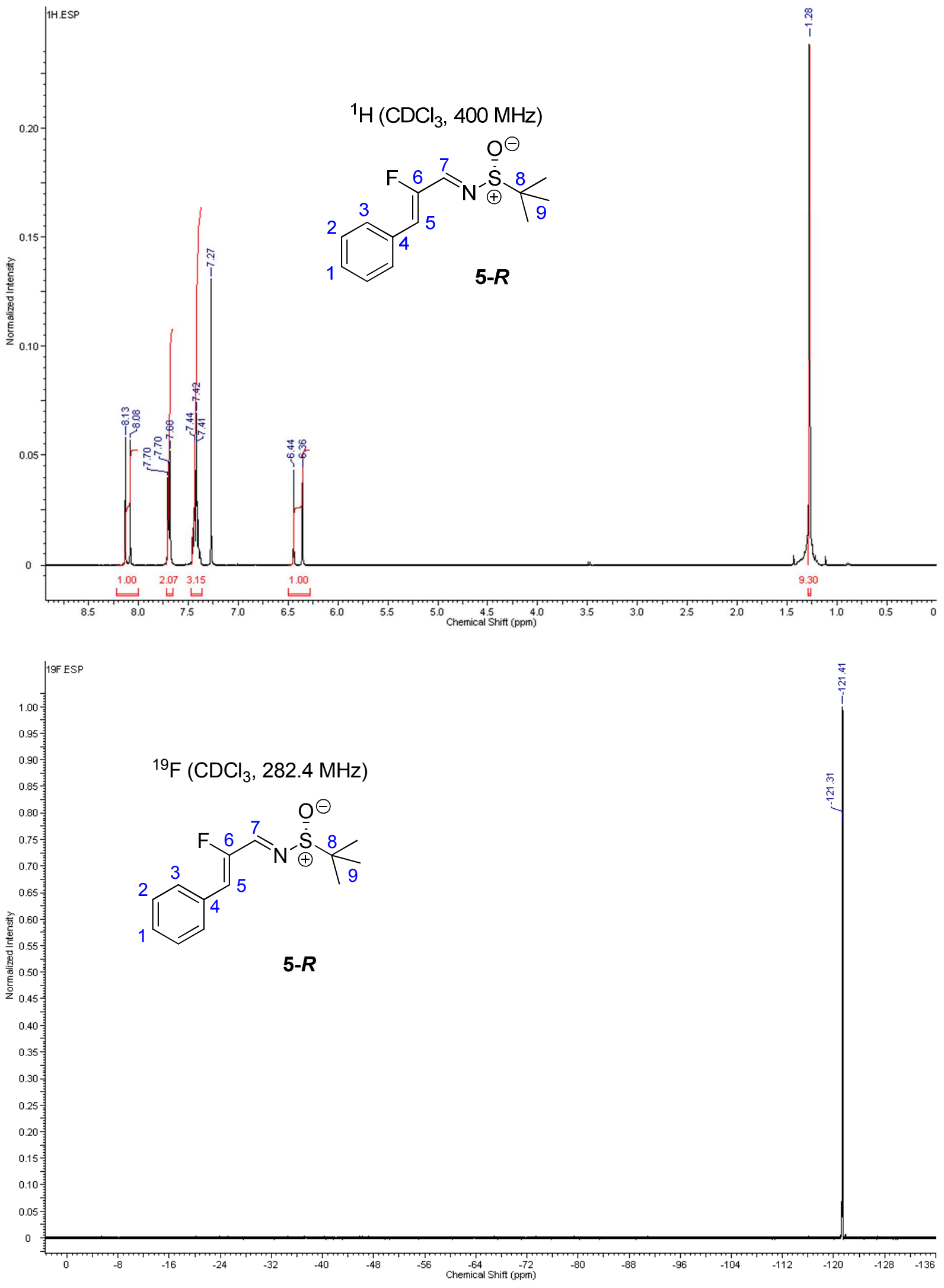


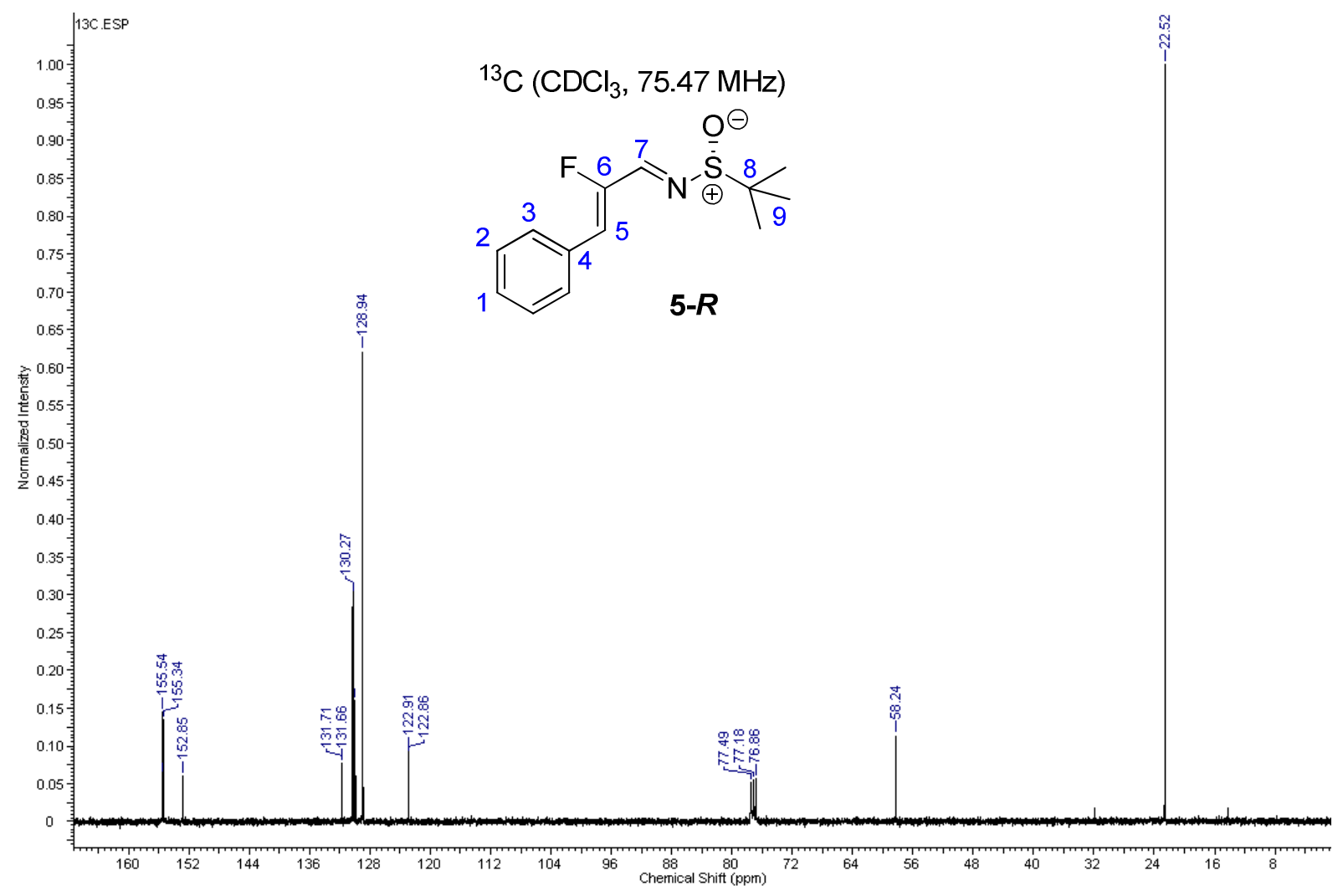

Compound 7b: (S,Z)-3-fluoro-N-(4-methoxybenzyl)-4-phenylbut-3-en-2-amine

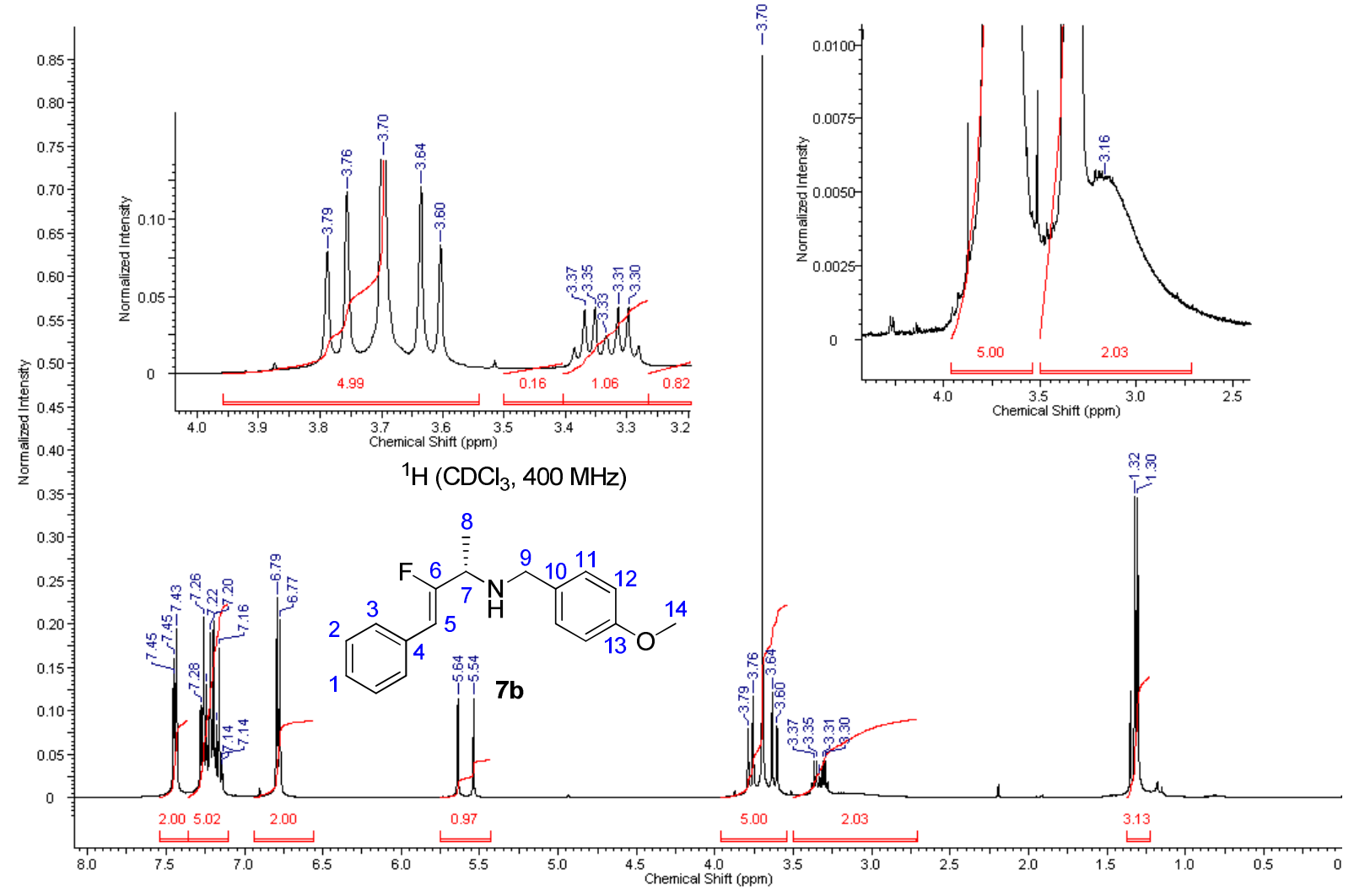



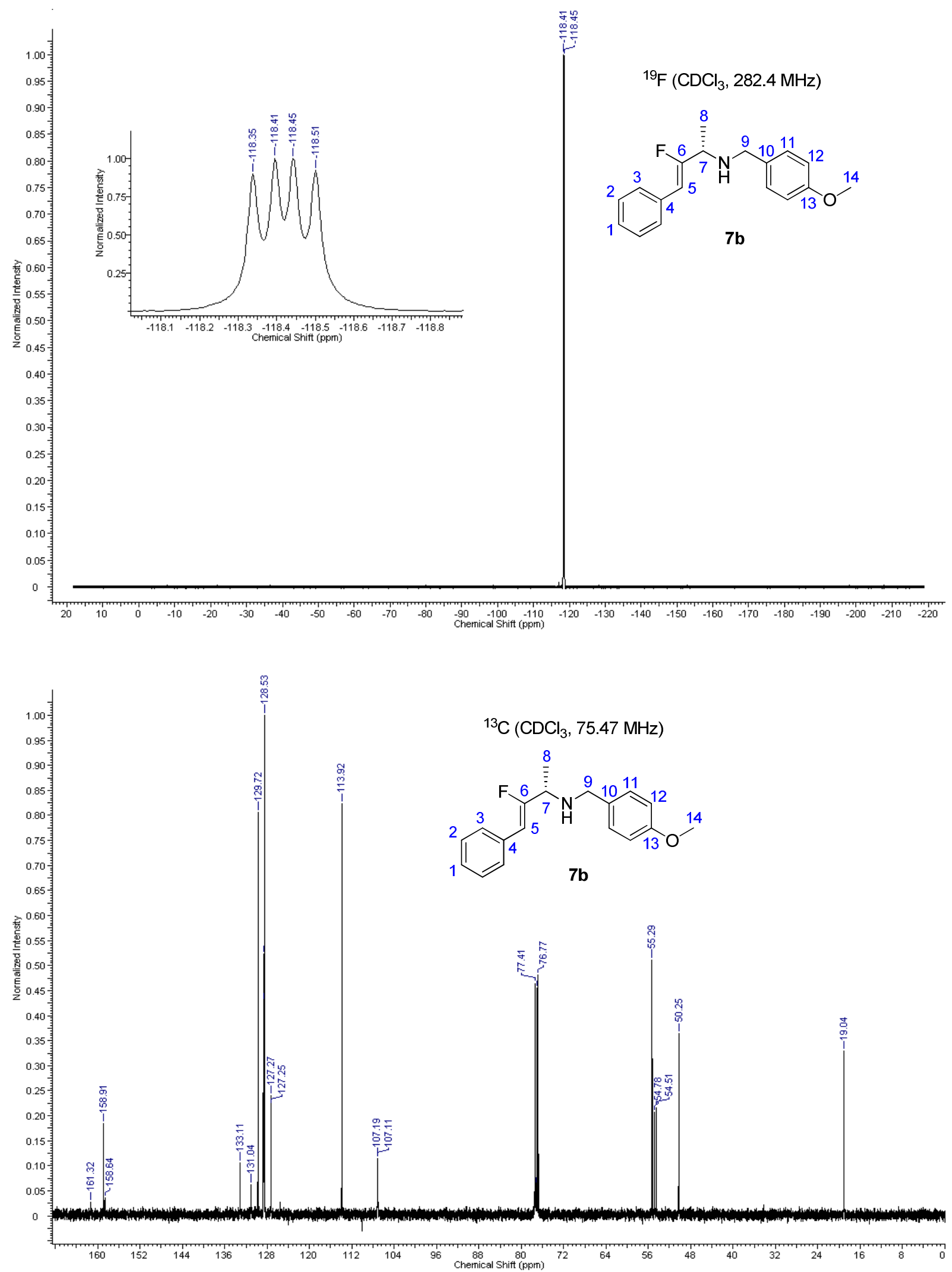
Compound 7c: (R,Z)-3-fluoro-N-(4-methoxybenzyl)-4-phenylbut-3-en-2-amine
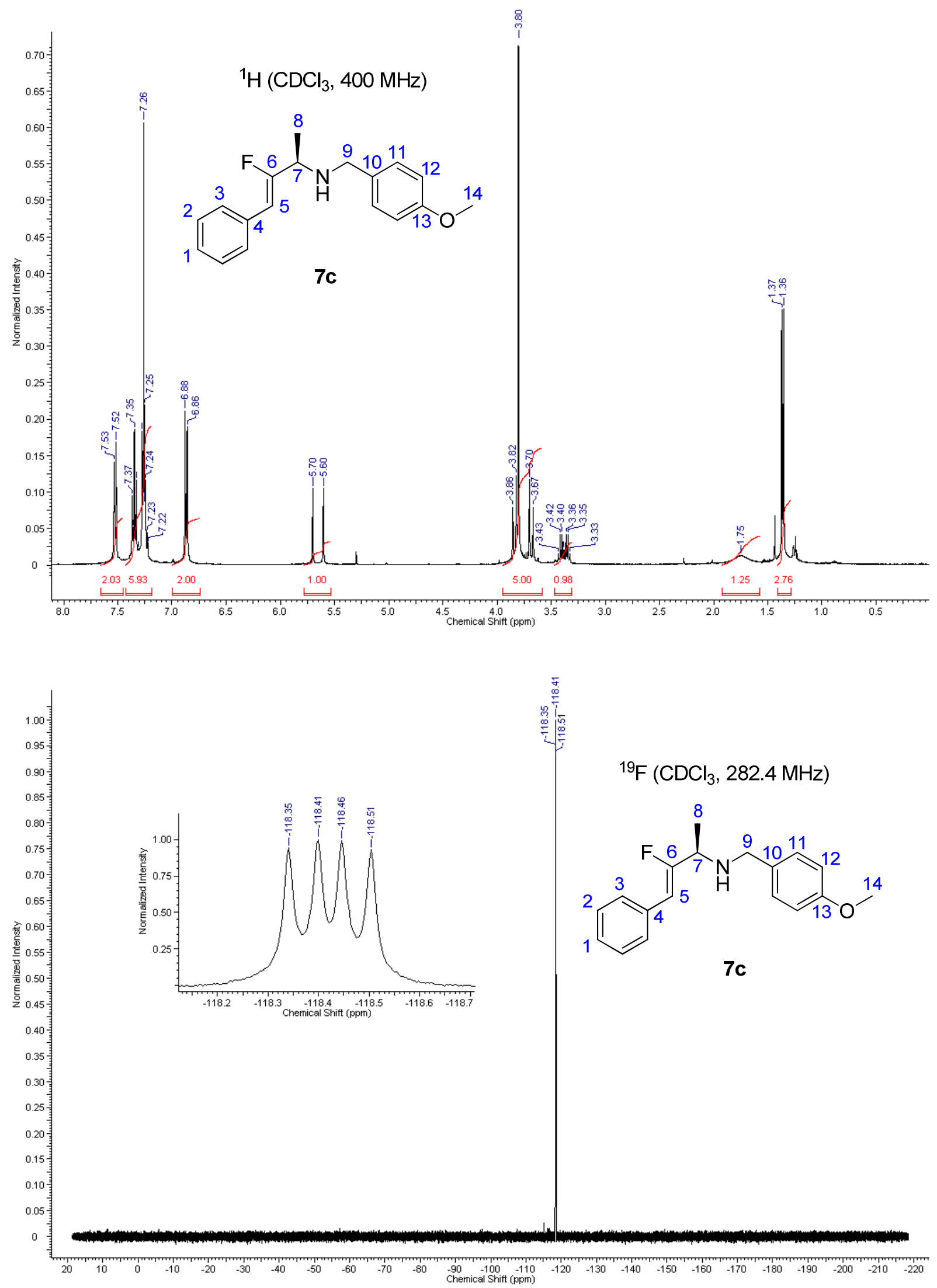


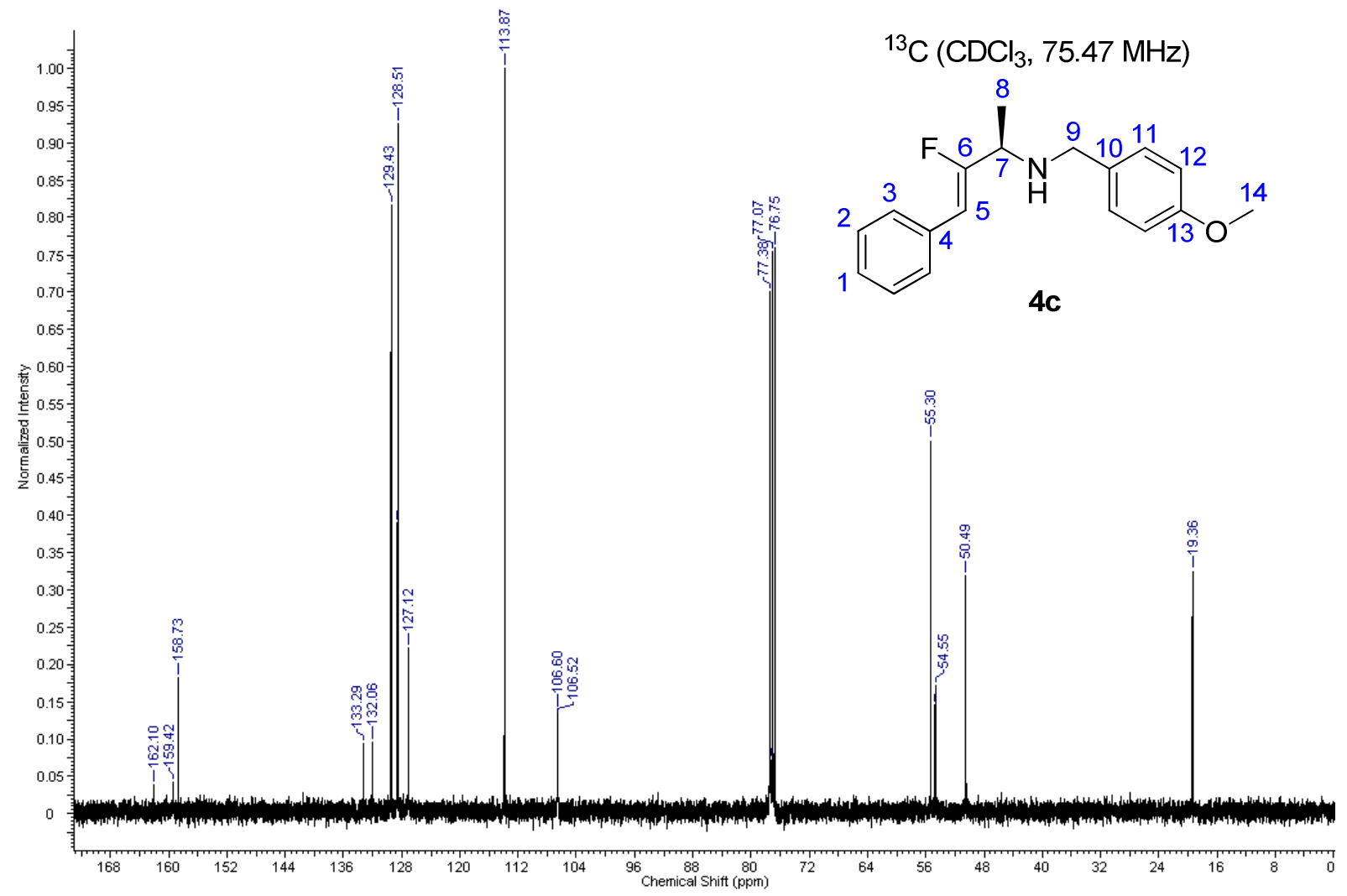

Compound 7d: (S,Z)-2-fluoro-N-(4-methoxybenzyl)-1,3-diphenylprop-2-en-1-amine

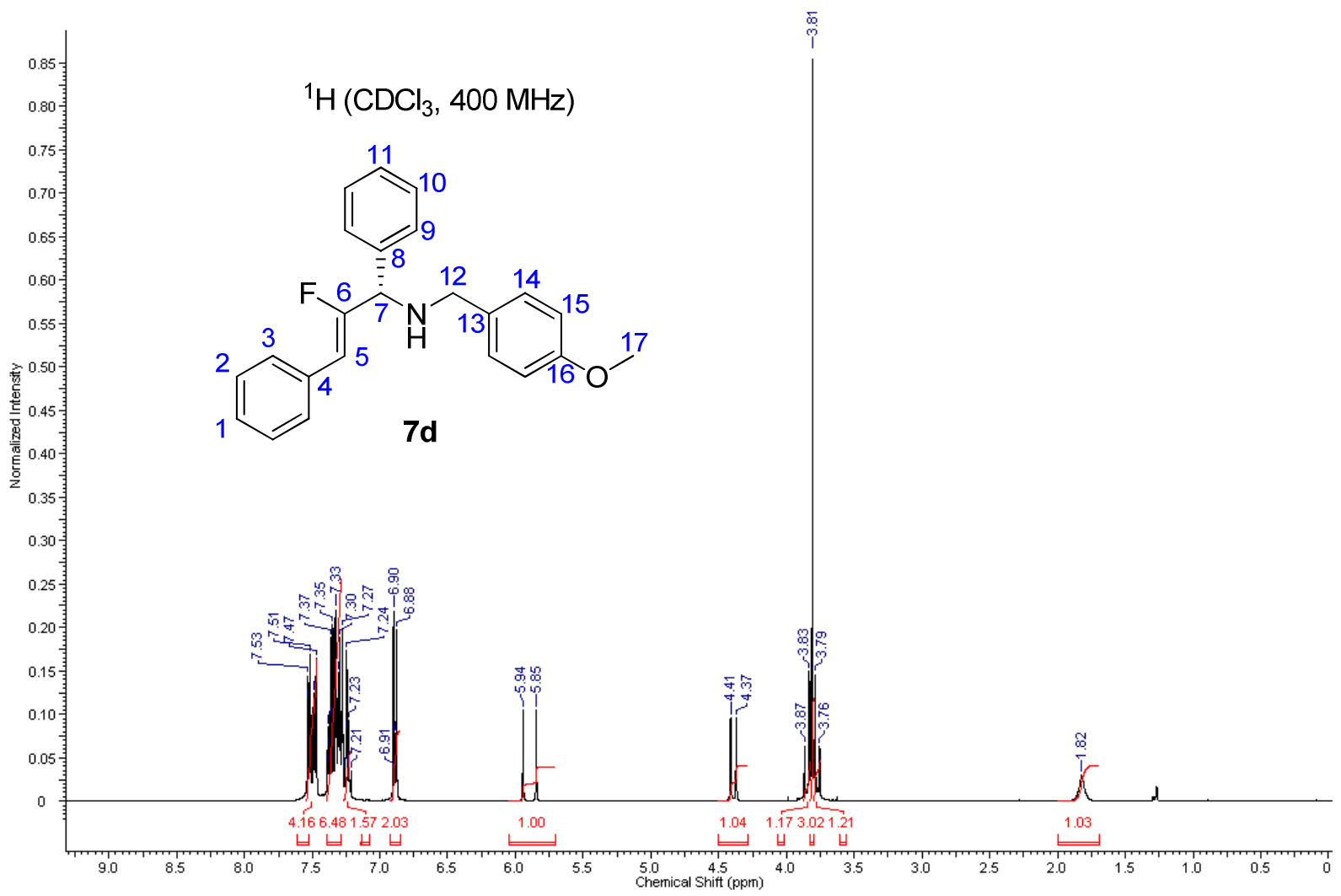



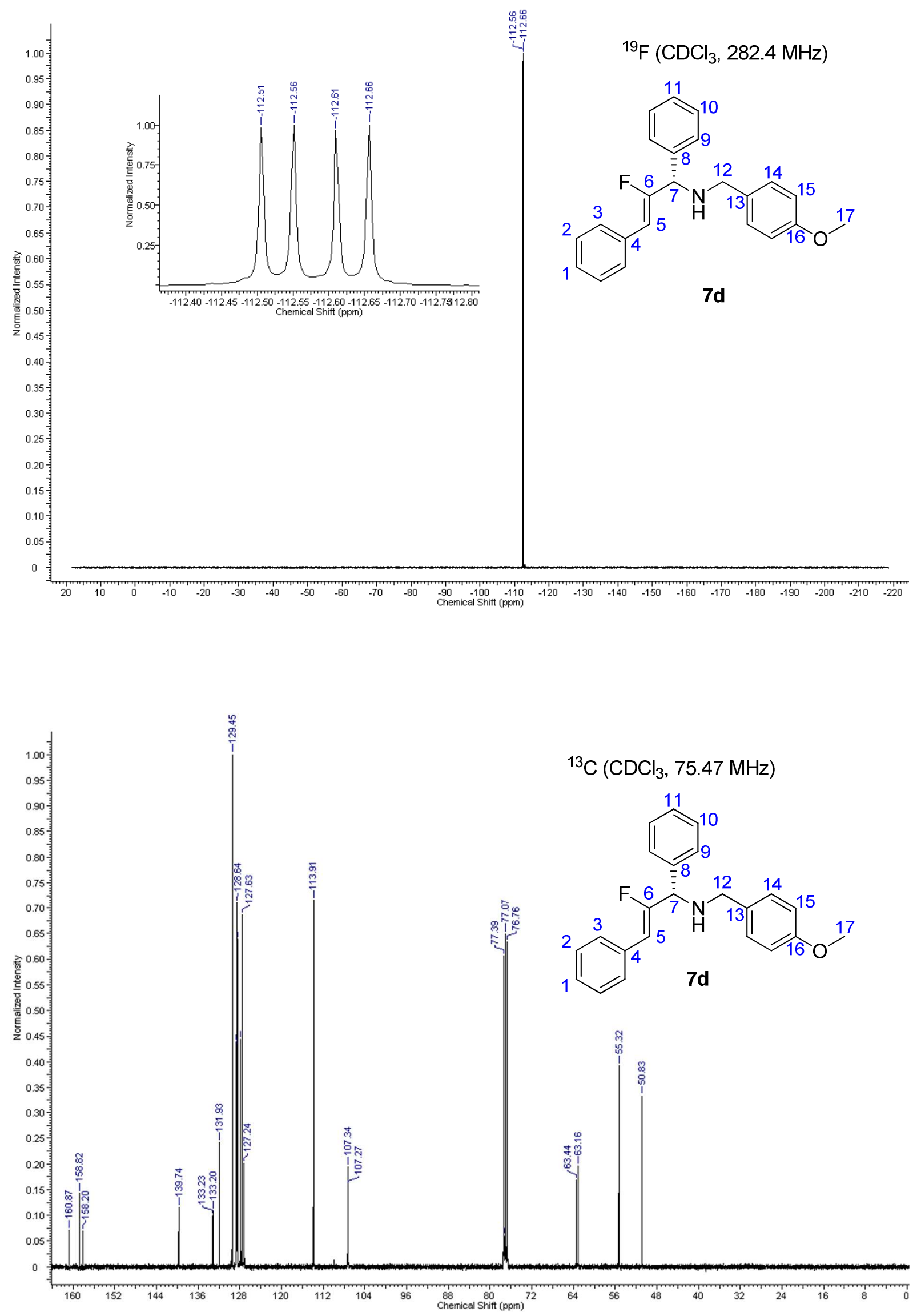
Compound 7d+e: (Z)-2-fluoro-N-(4-methoxybenzyl)-1,3-diphenylprop-2-en-1-amine
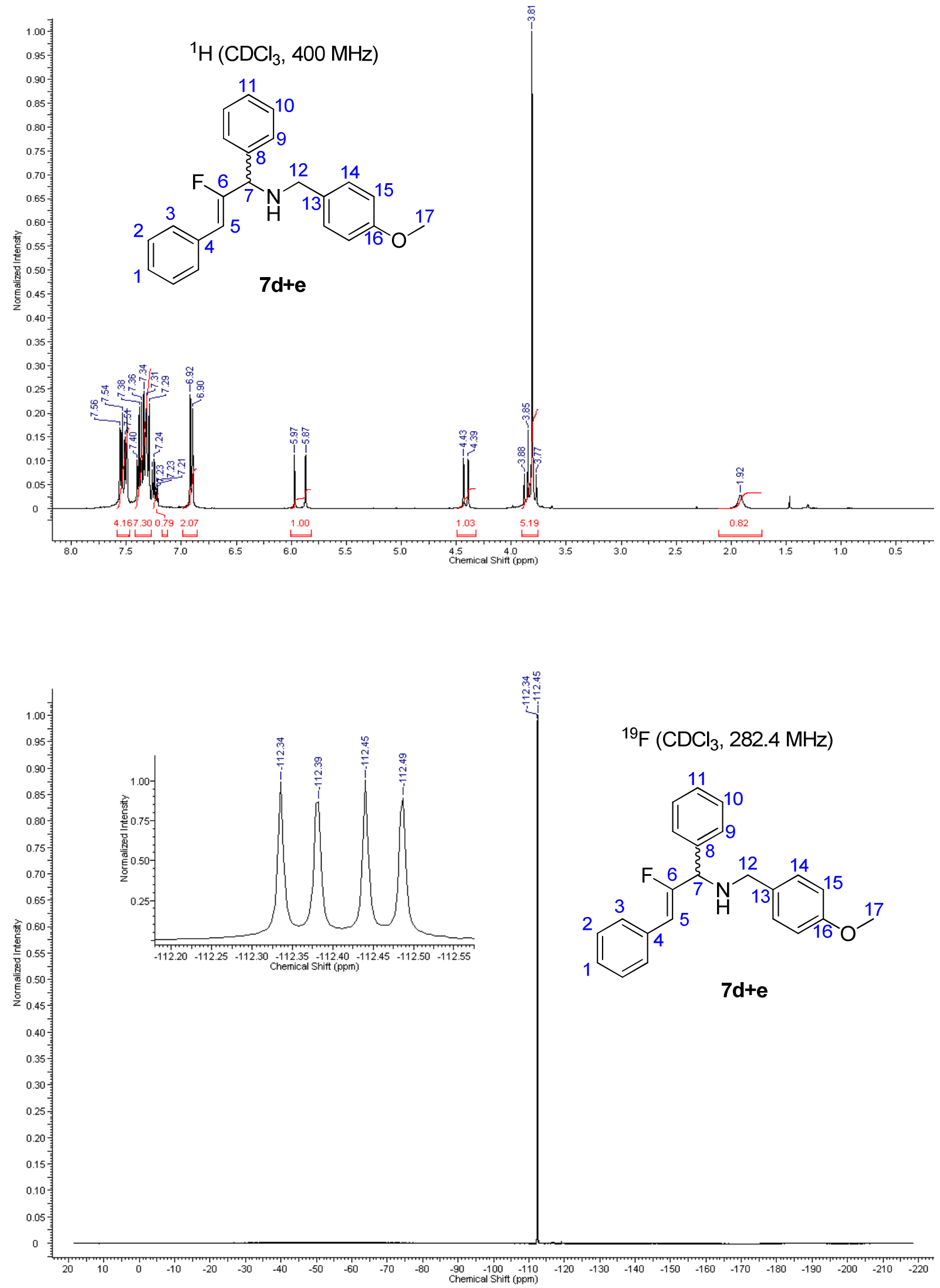


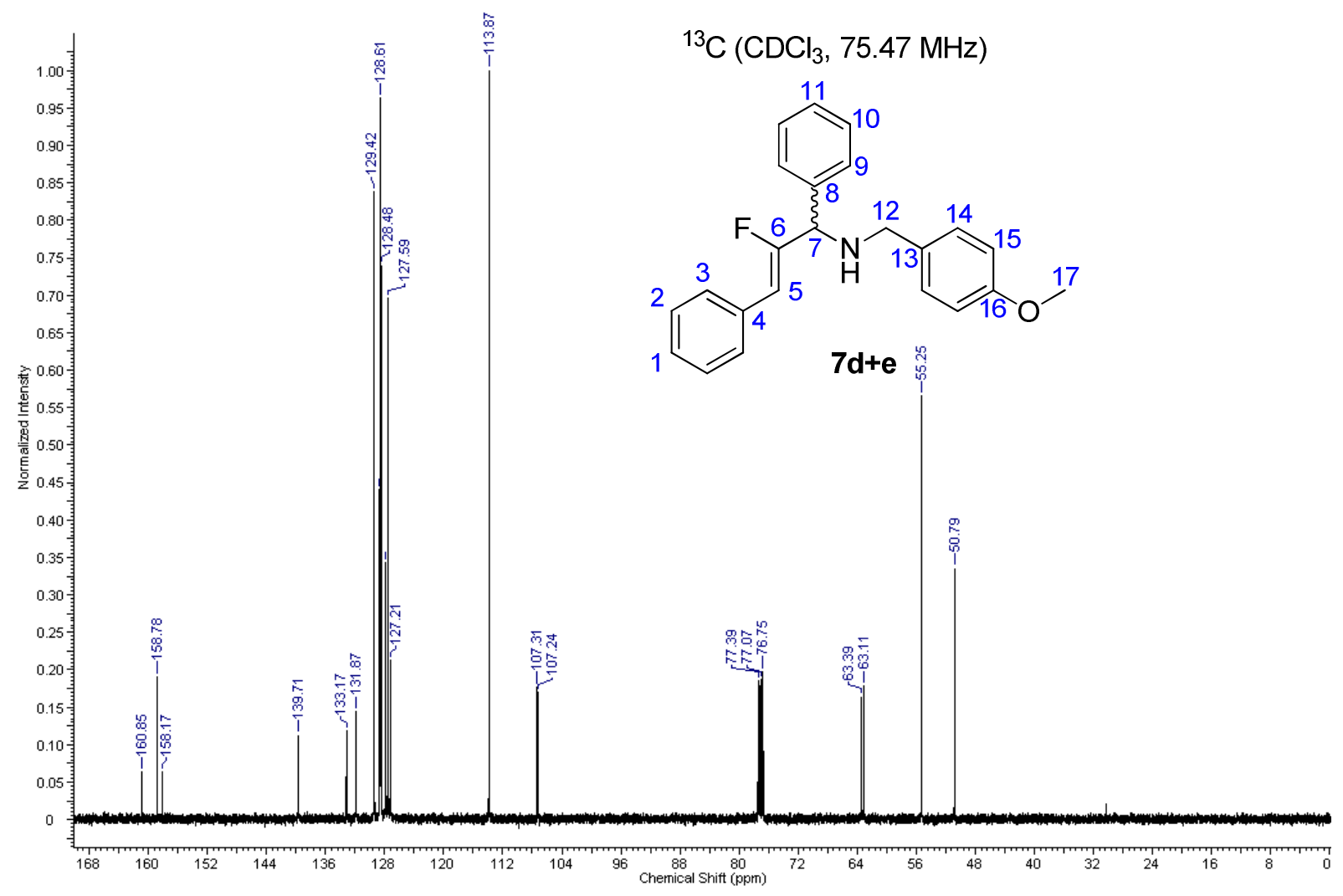

Compound 7f: (S,Z)-2-fluoro-N-(4-methoxybenzyl)-4-methyl-1-phenylpent-1-en-3-amine

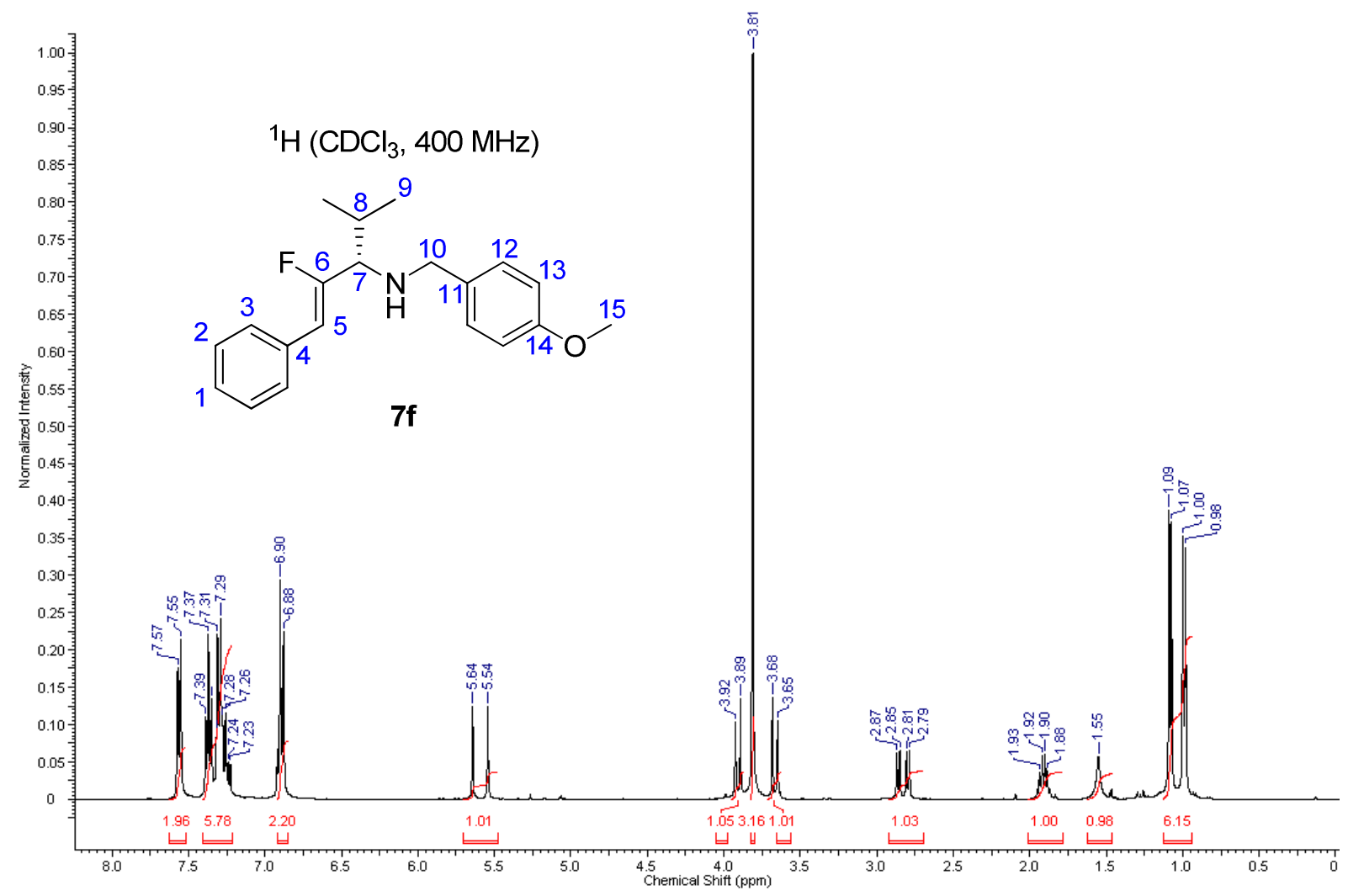



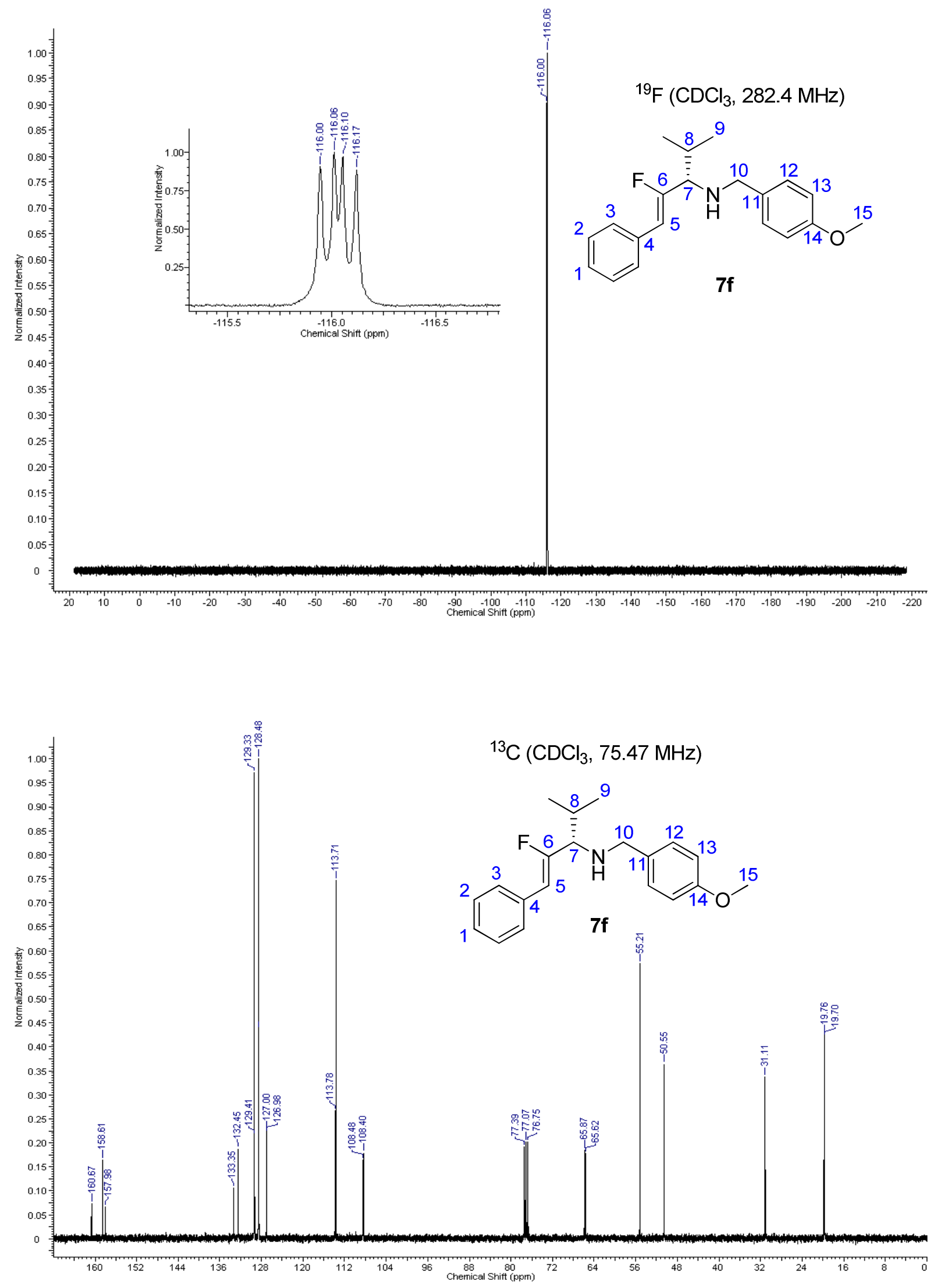

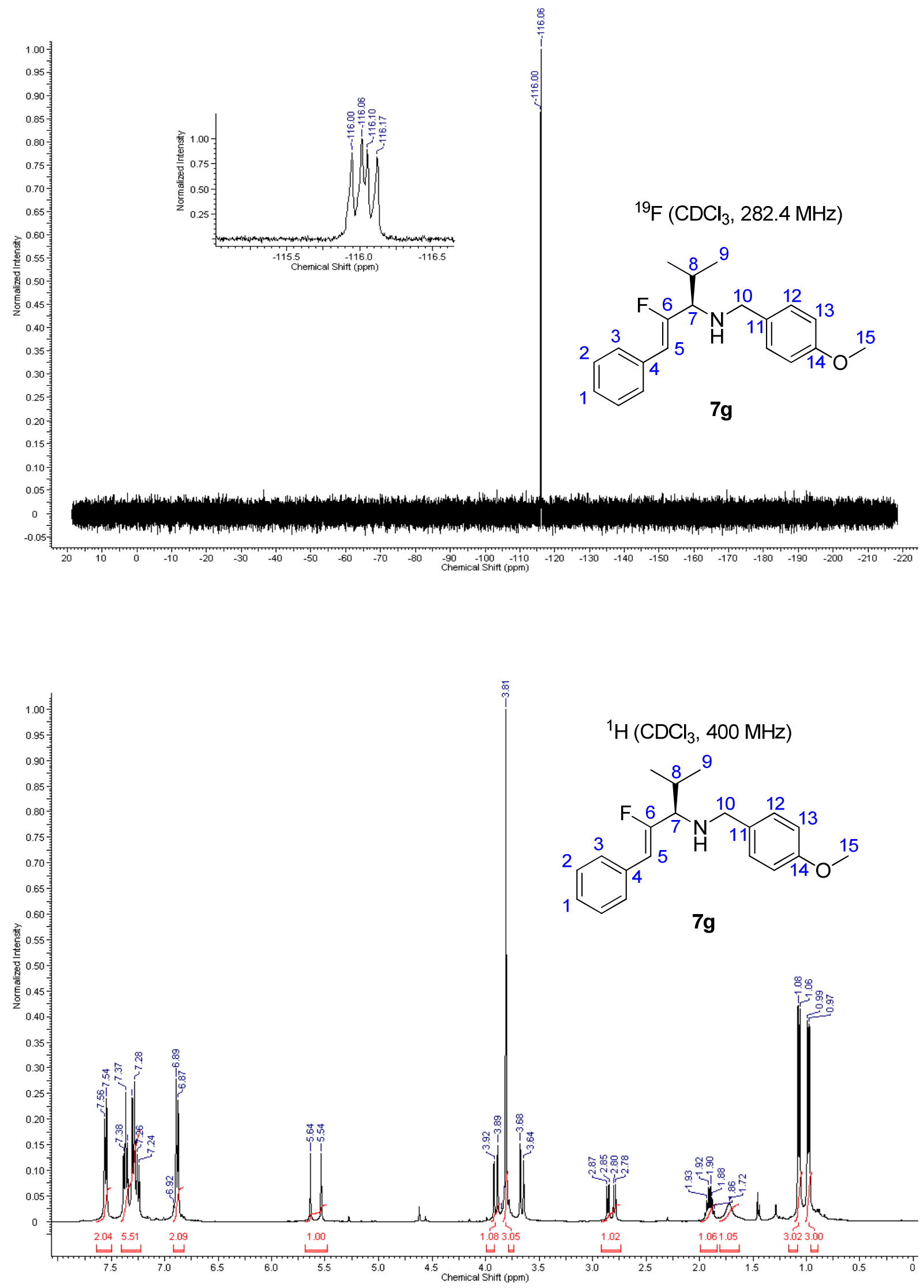


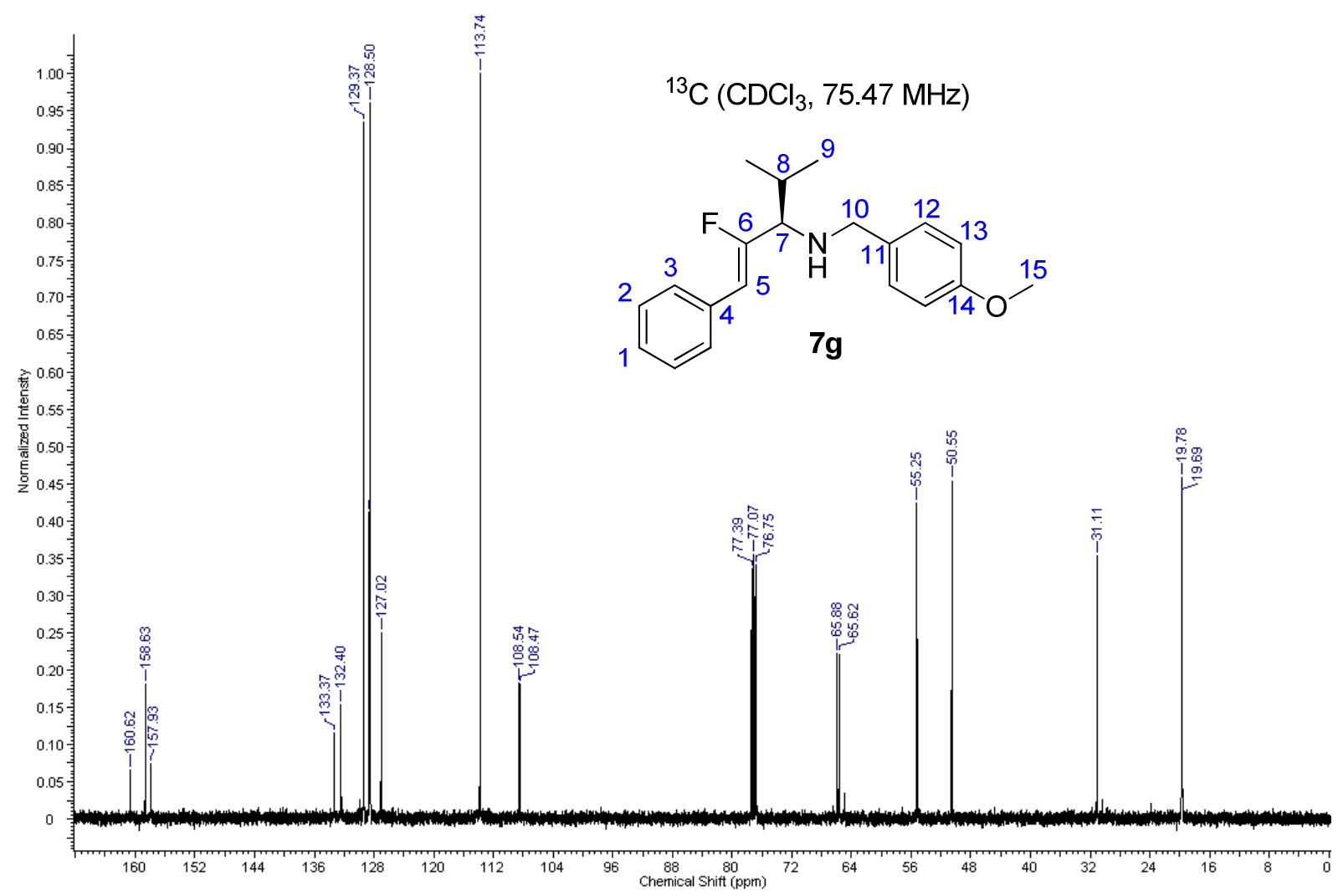

Compound 7h: (Z)-3-fluoro-N-(4-methoxybenzyl)-1,4-diphenylbut-3-en-2-amine

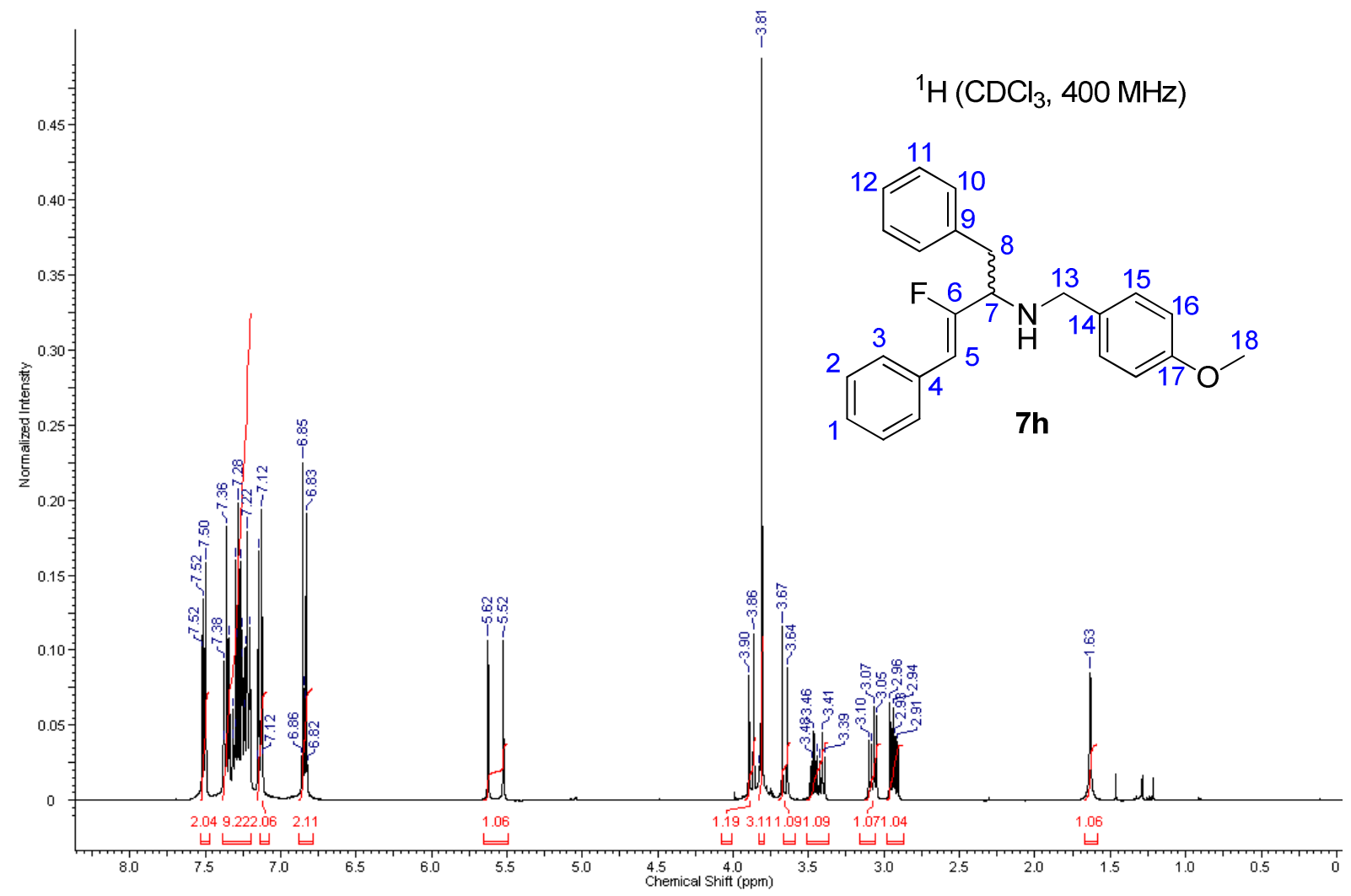



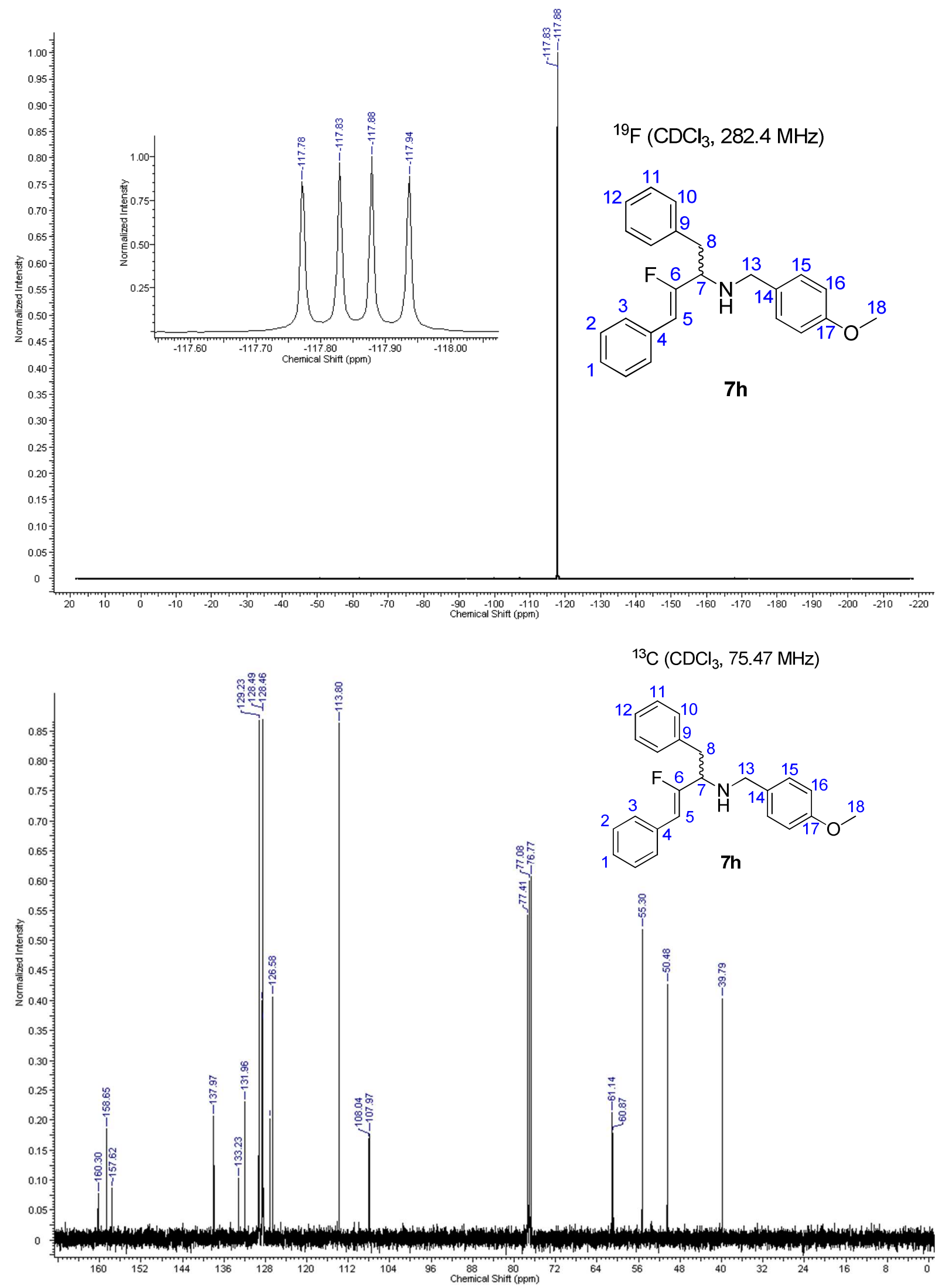
Compound 9c: (R,Z)-N-(3-fluoro-4-phenylbut-3-en-2-yl)but-3-enamide
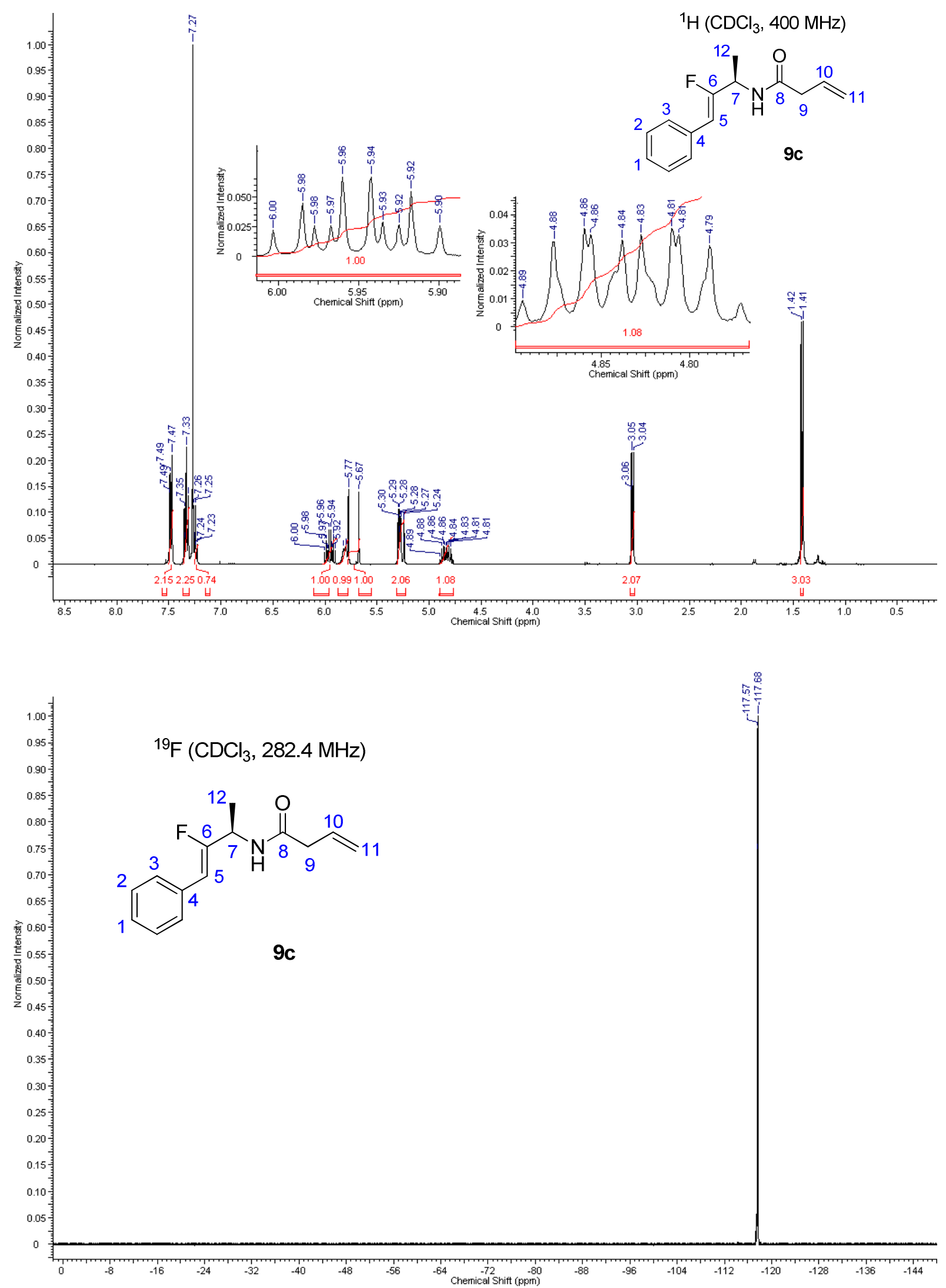


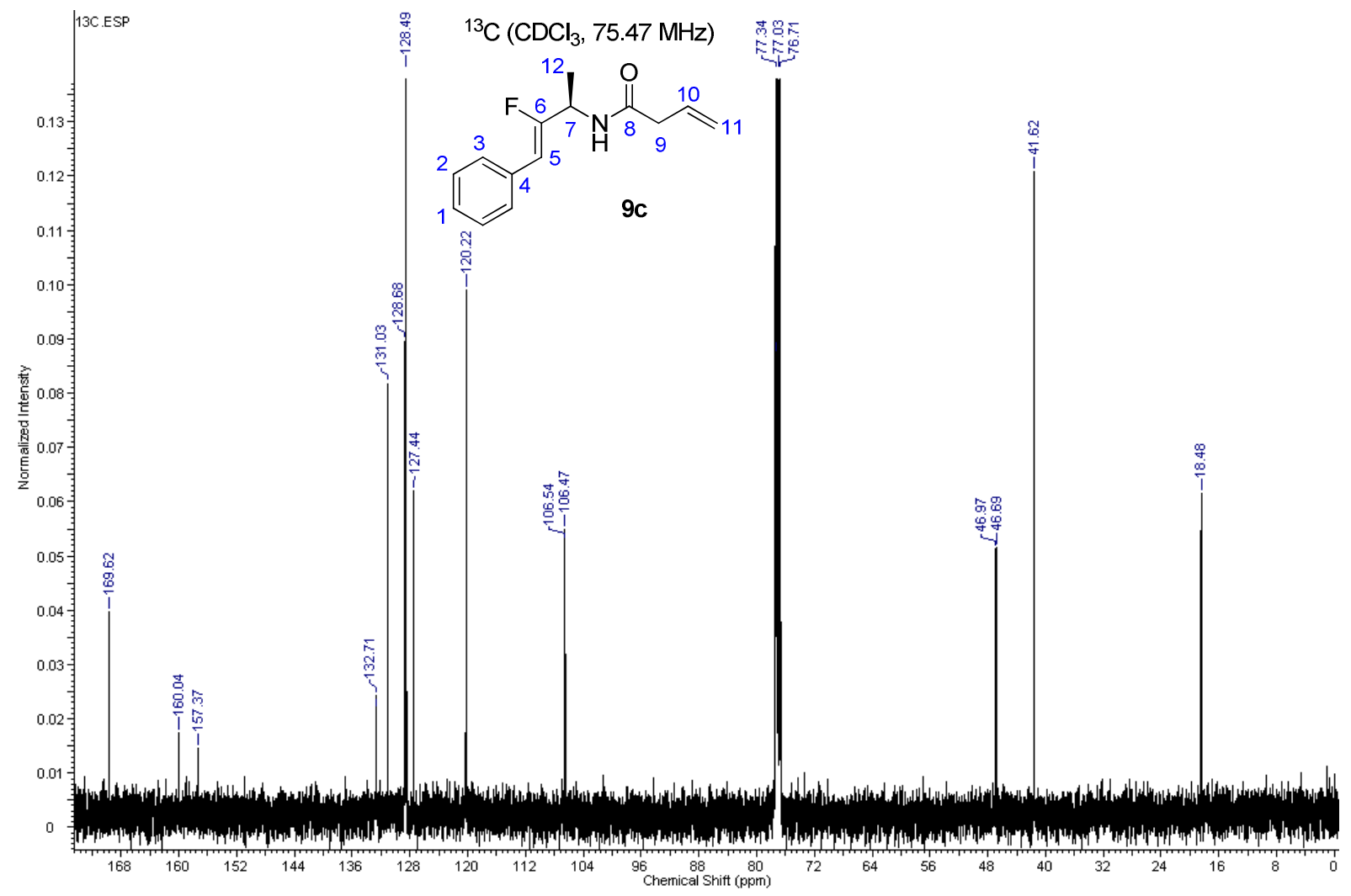

Compound 9e: (R,Z)-N-(2-fluoro-1,3-diphenylallyl)but-3-enamide

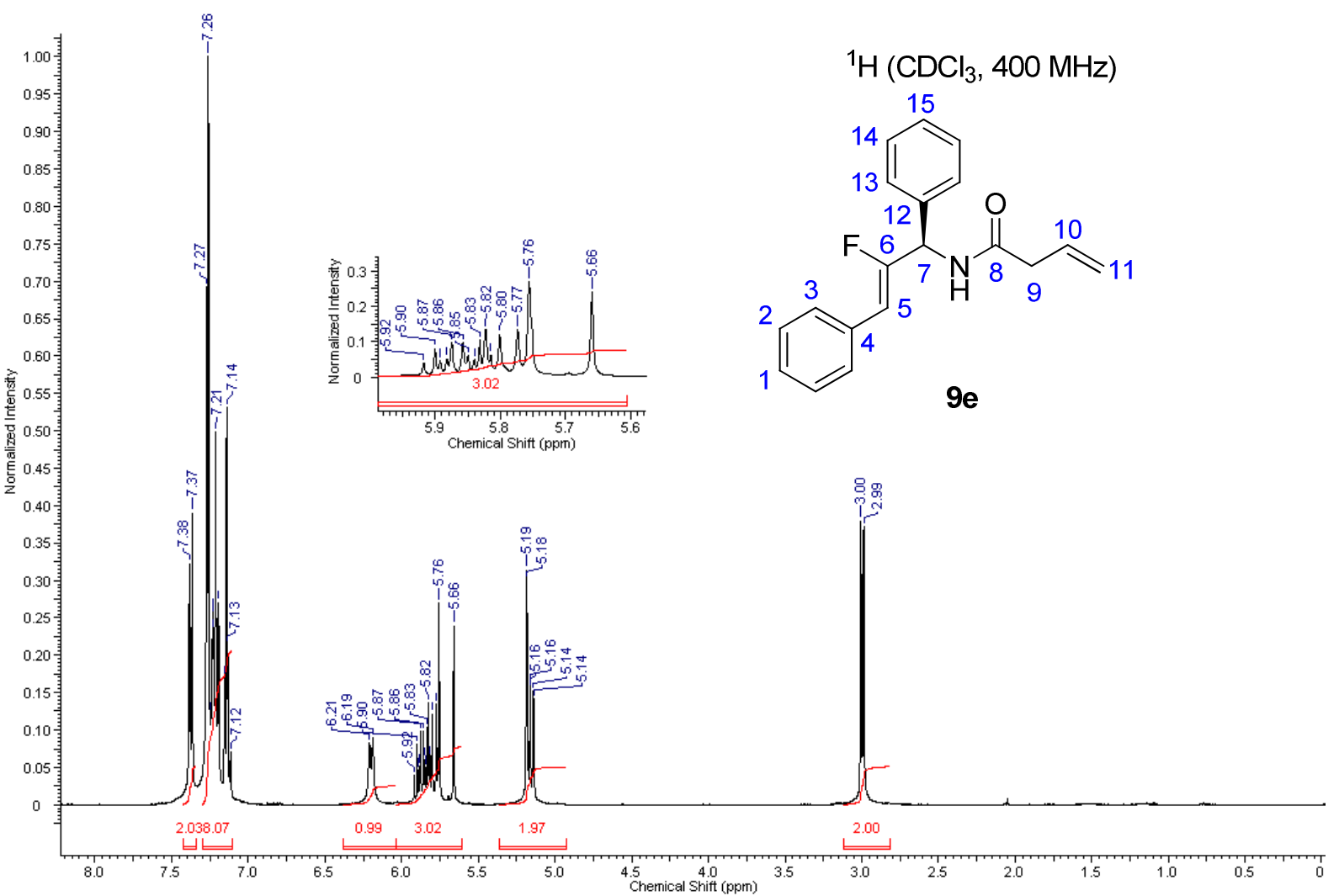



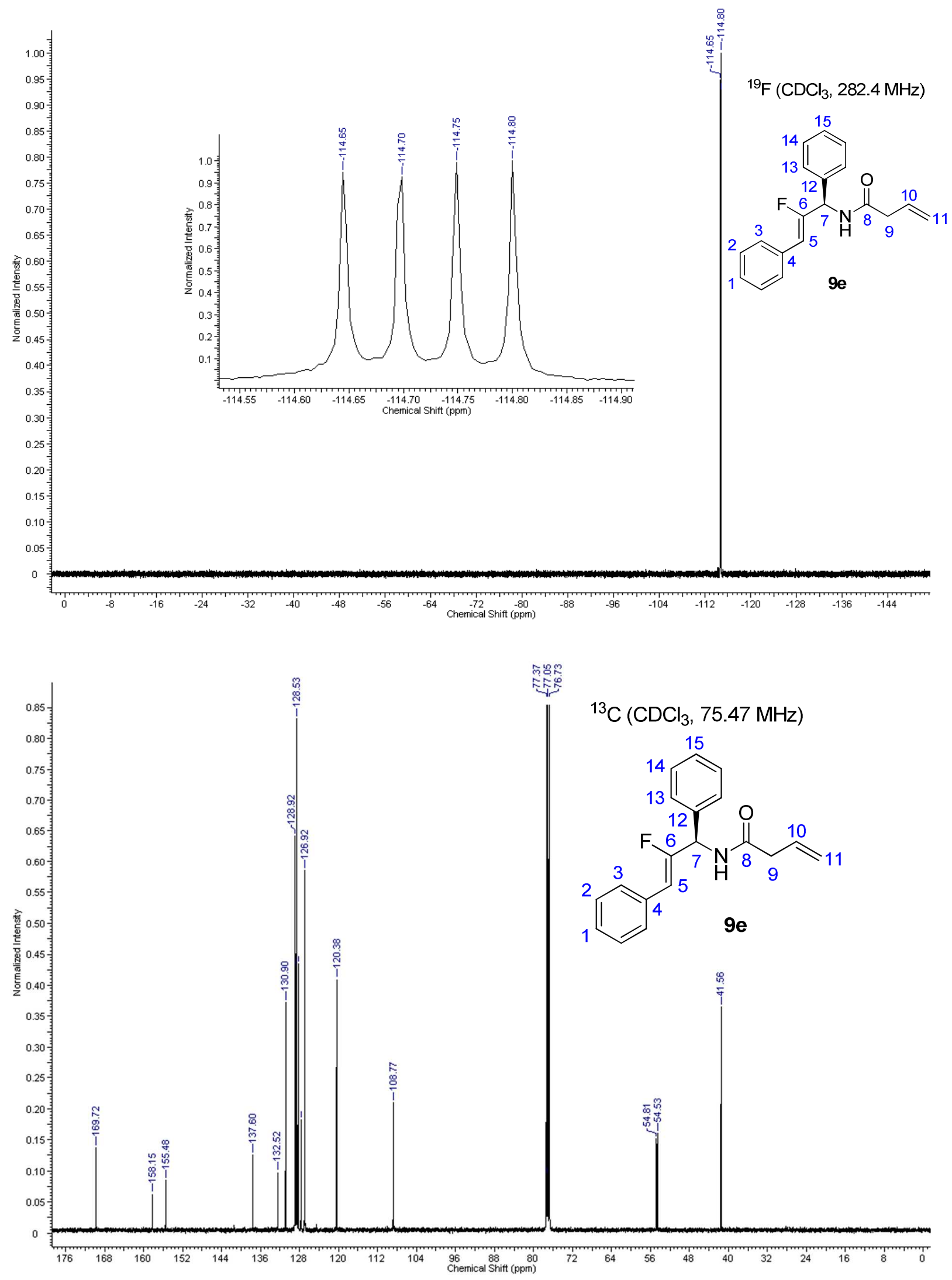

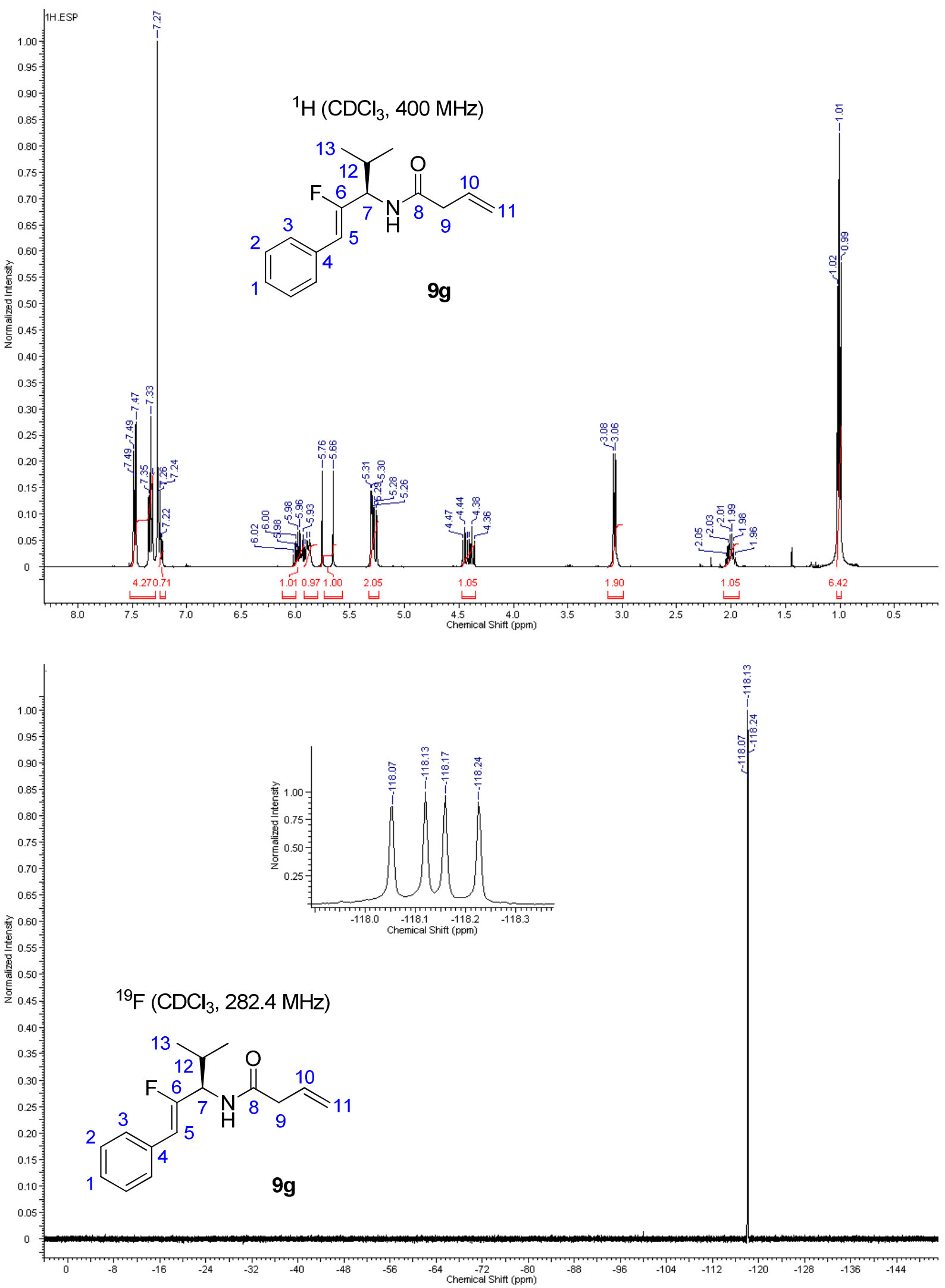


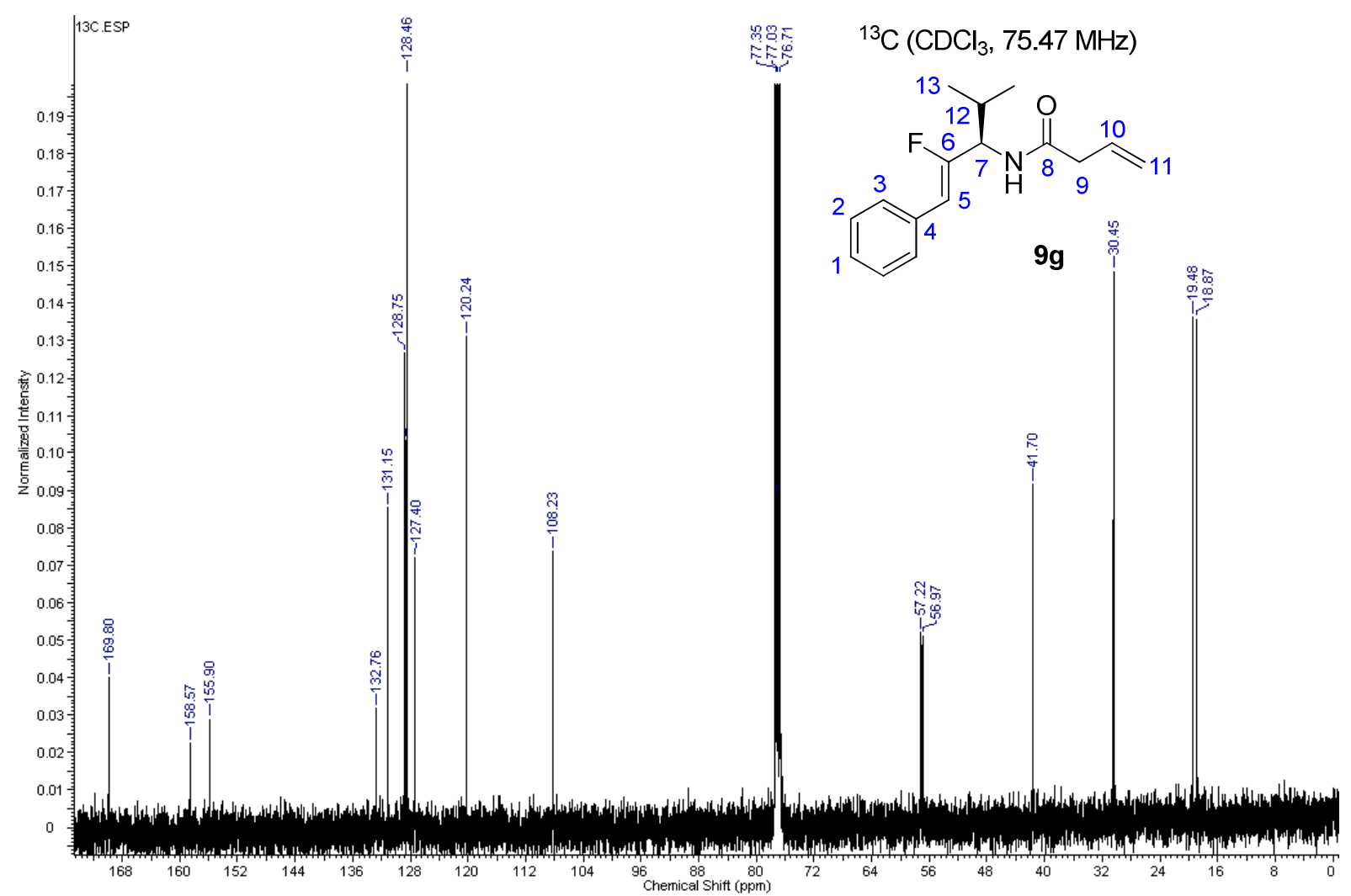

Compound 10a: 5-fluoro-1,6-dihydropyridin-2(3H)-one

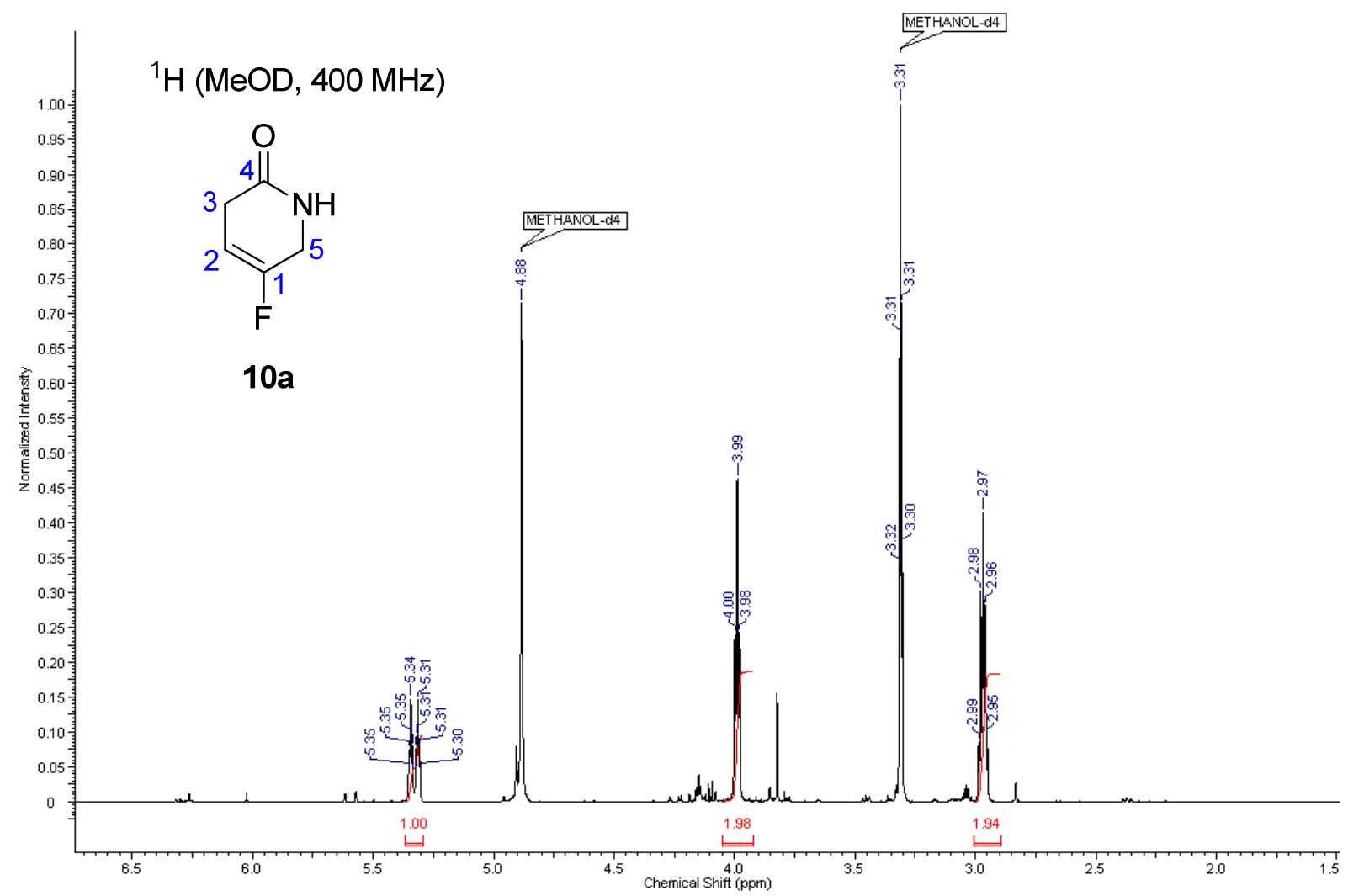



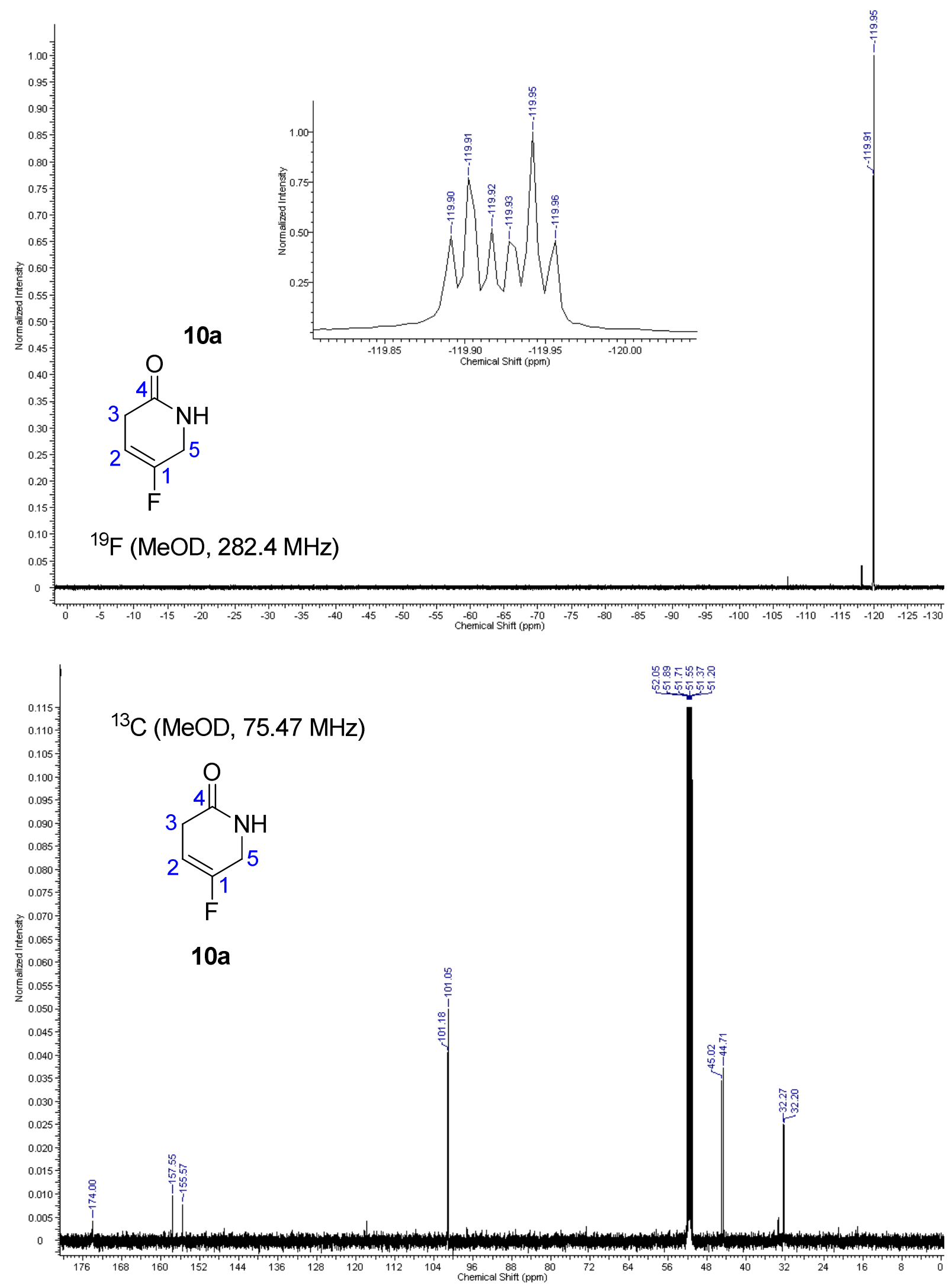
Compound 10d+e: 5-fluoro-6-phenyl-1,6-dihydropyridin-2(3H)-one
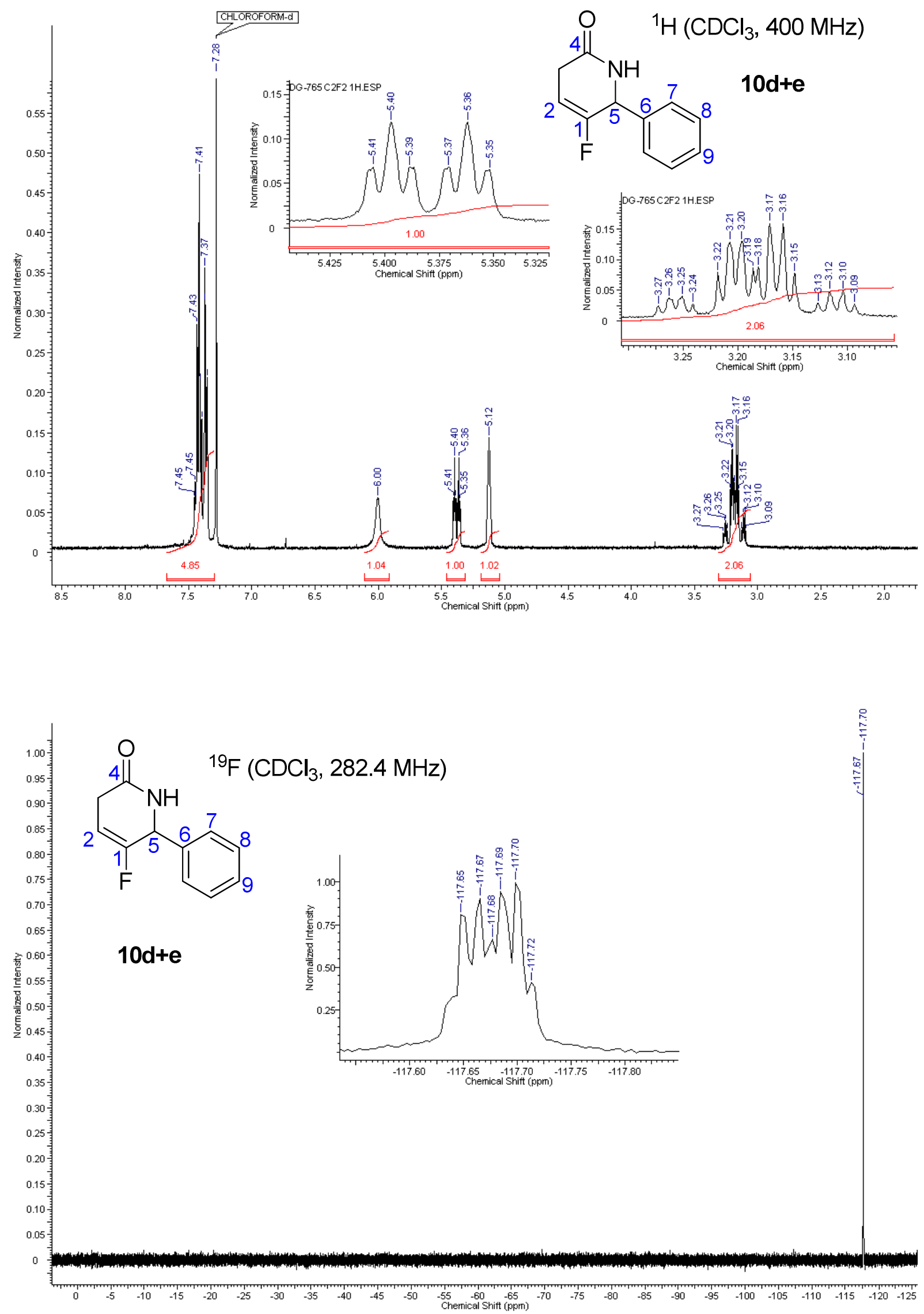
Compound 10d: (S)-5-fluoro-6-phenyl-1,6-dihydropyridin-2(3H)-one
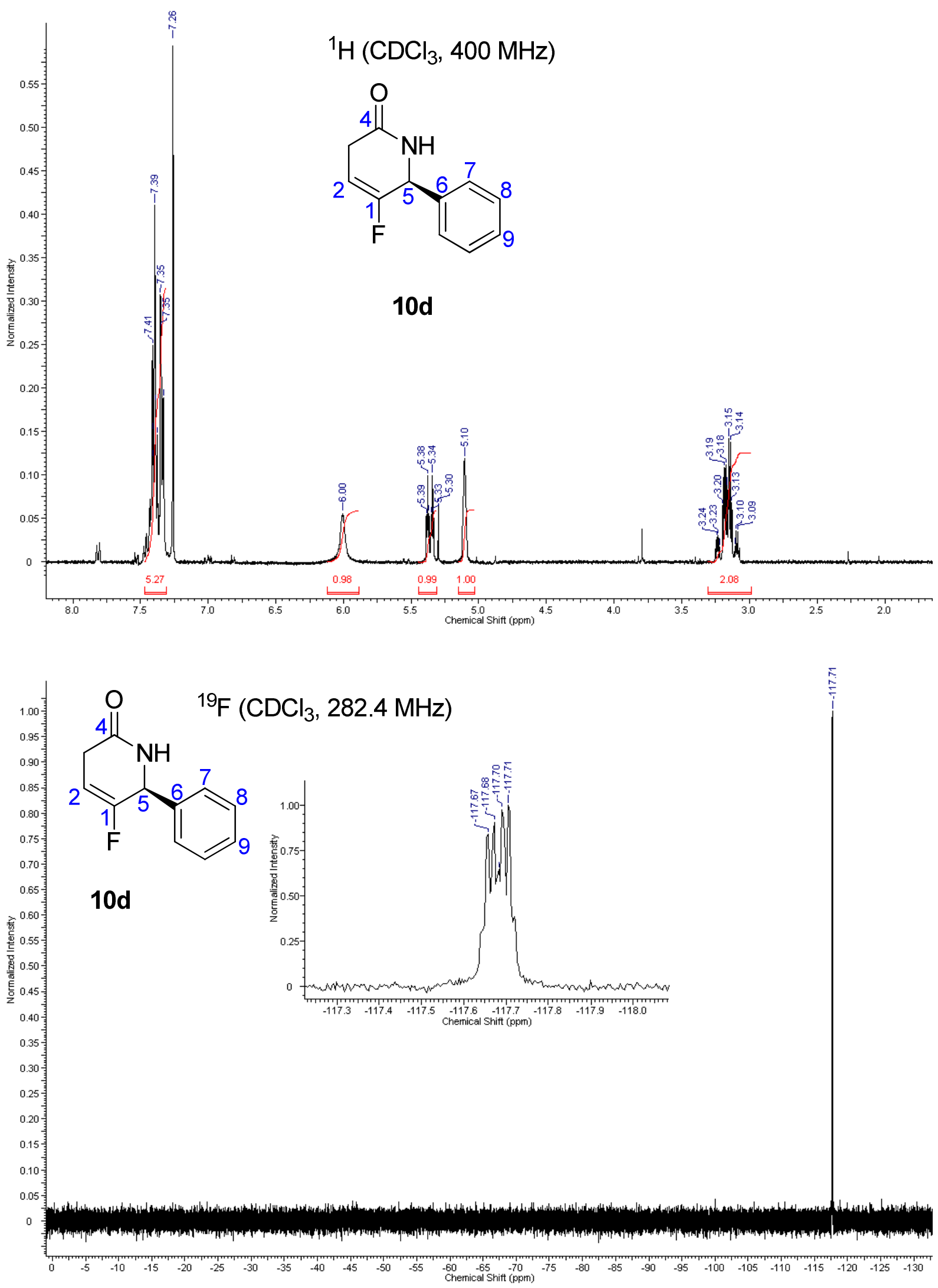


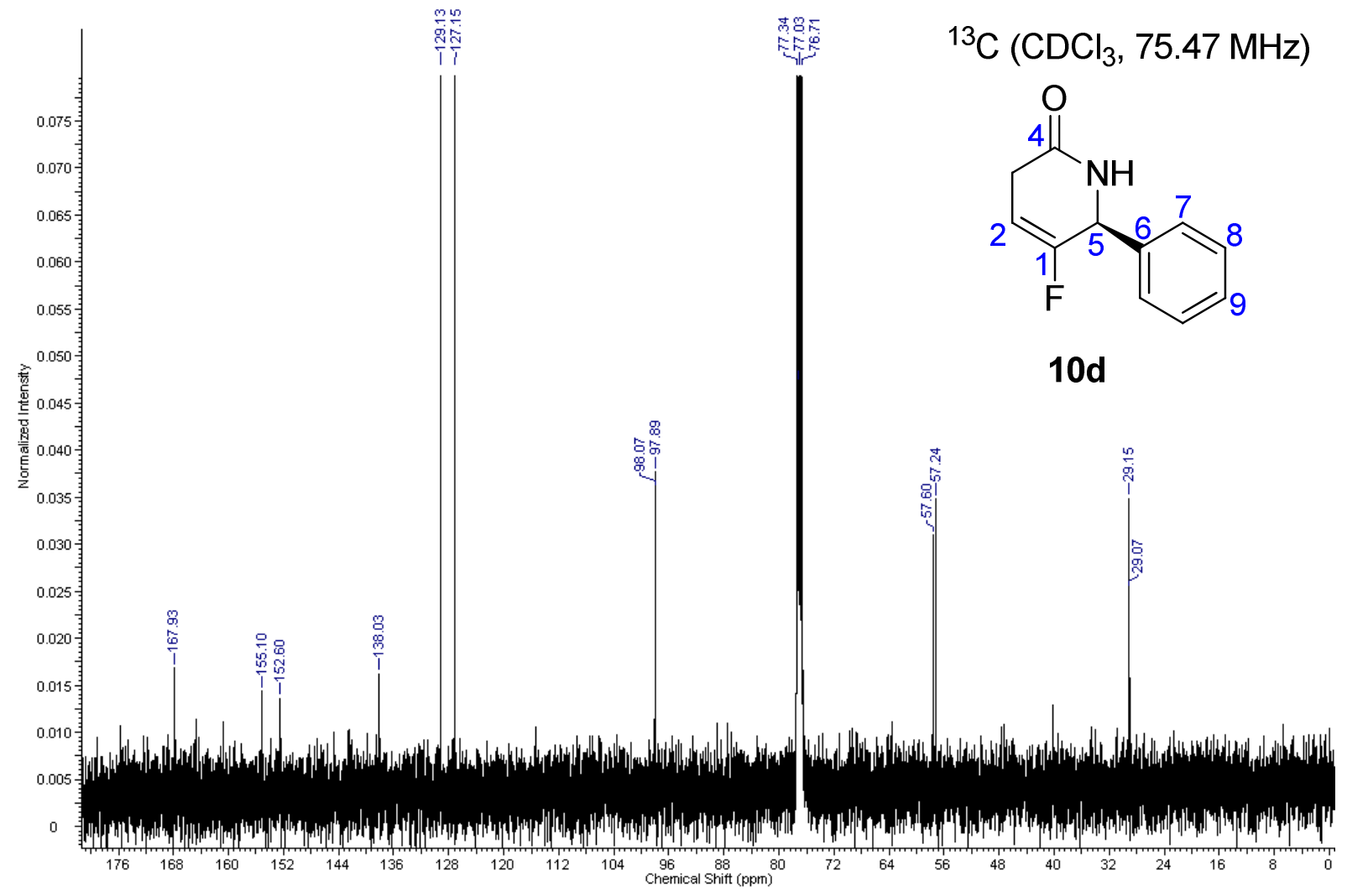

